Supporting Information

\title{
Rhodium-Catalyzed Remote Isomerization of Alkenyl Alcohols to Ketones
}

\author{
Wenke Dong, $\neq$ Hongxuan Yang, ${ }^{\prime}$ Wen Yang, ${ }^{*}$ and Wanxiang Zhao* \\ t State Key Laboratory of Chemo/Biosensing and Chemometrics, College of Chemistry and Chemical \\ Engineering, Hunan University, Changsha, Hunan 410082, China. \\ E-mail : zhaowanxiang@hnu.edu.cn. \\ E-mail: yangwen@hnu.edu.cn
}

\section{Contents}

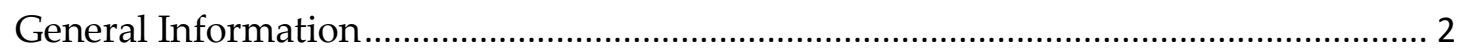

Optimization of the Reaction Conditions ................................................................ 3

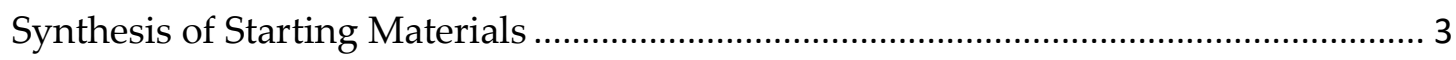

Rh-Catalyzed Remote Isomerization of Alkenyl Alcohols.......................................... 27

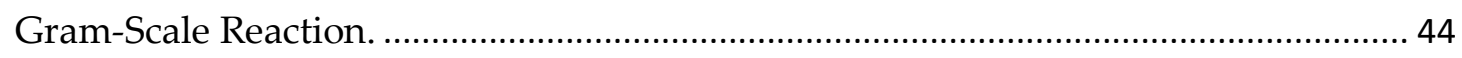

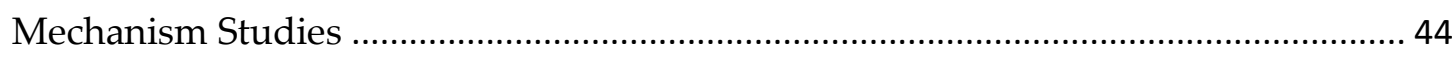

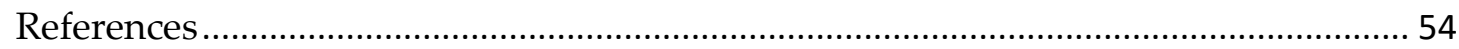

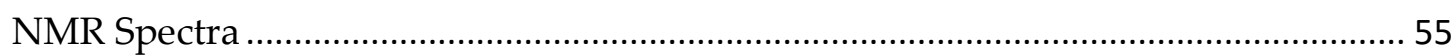




\section{General Information}

Unless otherwise noted, all reactions were conducted in oven-dried flasks or vials with a magnetic stirrer under nitrogen atmosphere. Solvents were purified under nitrogen using a solvent purification system. Analytical thin layer chromatography (TLC) was performed using silica gel plates. Visualisation was by ultraviolet fluorescence, and/or phosphomolybdic acid, and/or $\mathrm{KMnO}_{4}$. Flash column chromatography was performed using EM Science (200-300 mesh) silica gel.

${ }^{1} \mathrm{H}$ NMR spectra were recorded using a Bruker $400 \mathrm{MHz}$ instrument with tetramethylsilane (TMS) as an internal standard. ${ }^{13} \mathrm{C}$ NMR spectra were obtained at $100 \mathrm{MHz}$ and referenced to the internal solvent signals. The data are reported as follows: chemical shift (ppm), multiplicity ( $\mathrm{s}=$ singlet, $\mathrm{d}=$ doublet, $\mathrm{dd}=$ doublet of doublet, $\mathrm{t}=$ triplet, $\mathrm{m}=$ multiplet, $\mathrm{br}=$ broad), coupling constant $J(\mathrm{~Hz})$, and integration. High resolution mass spectra were recorded on a Bruker Maxis System. IR spectra were collected on a Spectrum QATR-S from SHIMADZU and reported in unit of $\mathrm{cm}^{-1}$.

Commercially available reagents and solvents were used without further purification. $[\mathrm{RhCl}(\mathrm{cod})]_{2}$ was prepared according to literature procedure and all characterization data are in accordance with the literature. ${ }^{1} \mathrm{RhCl}$ (Xantphos) was prepared by literature procedures. ${ }^{2}$ 


\section{Optimization of the Reaction Conditions}

\begin{tabular}{|c|c|c|c|c|c|}
\hline & $\mathbf{a}(0.3 \mathrm{mmol})$ & $\begin{array}{c}\begin{array}{c}{[\mathrm{Rh}](4 \mathrm{~mol} \%)} \\
\mathrm{L}(4 \mathrm{~mol} \%)\end{array} \\
\underset{\text { base }(5 \mathrm{~mol} \%)}{\text { solvent }(1.0 \mathrm{~mL})} \\
70^{\circ} \mathrm{C}, 15 \mathrm{~h}\end{array}$ & ${ }_{\mathbf{2 a}}^{\mathrm{O}}$ & $\mathbf{a}$ & \\
\hline entry & {$[\mathrm{Rh}]$} & ligand & solvent & base & $\mathbf{2 a} / \mathbf{3} \mathbf{a}$ yield $(\%)^{a}$ \\
\hline 1 & {$[\mathrm{RhCl}(\operatorname{cod})]_{2}$} & Xantphos & THF & $\mathrm{K}_{2} \mathrm{CO}_{3}$ & $43 / 57$ \\
\hline 2 & {$[\mathrm{RhCl}(\operatorname{cod})]_{2}$} & Xantphos & THF & DABCO & $17 / 83$ \\
\hline 3 & {$[\mathrm{RhCl}(\operatorname{cod})]_{2}$} & Xantphos & THF & $\mathrm{NaF}$ & $3 / 97$ \\
\hline 4 & {$[\mathrm{RhCl}(\operatorname{cod})]_{2}$} & Xantphos & THF & $\mathrm{CsOAc}$ & $>99 /<1$ \\
\hline 5 & {$[\mathrm{RhCl}(\operatorname{cod})]_{2}$} & Xantphos & THF & - & $4 / 96$ \\
\hline 6 & {$[\mathrm{RhCl}(\mathrm{cod})]_{2}$} & Xantphos & 1,4-dioxane & $\mathrm{CsOAc}$ & $80 / 20$ \\
\hline 7 & {$[\mathrm{RhCl}(\operatorname{cod})]_{2}$} & Xantphos & DMA & $\mathrm{CsOAc}$ & $<1 />99$ \\
\hline 8 & {$[\mathrm{RhCl}(\operatorname{cod})]_{2}$} & Xantphos & EtOAc & $\mathrm{CsOAc}$ & $54 / 46$ \\
\hline 9 & {$[\mathrm{RhCl}(\operatorname{cod})]_{2}$} & Cy-Xantphos & THF & $\mathrm{CsOAc}$ & $8 / 92$ \\
\hline 10 & {$[\mathrm{RhCl}(\mathrm{cod})]_{2}$} & $\mathrm{PPh}_{3}$ & THF & $\mathrm{CsOAc}$ & $50 / 50$ \\
\hline 11 & {$\left[\mathrm{RhCl}\left(\mathrm{C}_{2} \mathrm{H}_{4}\right)\right]_{2}$} & Xantphos & THF & $\mathrm{CsOAc}$ & $<5 / 78$ \\
\hline 12 & $\mathrm{RhCl}\left(\mathrm{PPh}_{3}\right)_{3}$ & Xantphos & THF & $\mathrm{CsOAc}$ & $24 / 69$ \\
\hline 13 & $\mathrm{RhCl(Xantphos)}$ & - & THF & $\mathrm{CsOAc}$ & $>99 /<1$ \\
\hline 14 & {$[\mathrm{Rh}(\mathrm{OMe})(\mathrm{cod})]_{2}$} & Xantphos & THF & - & $>99 /<1$ \\
\hline 15 & {$[\mathrm{Rh}(\mathrm{OAc})(\operatorname{cod})]_{2}$} & Xantphos & THF & - & $>99 /<1$ \\
\hline
\end{tabular}

${ }^{a}$ Determined by ${ }^{1} \mathrm{H}$ NMR using $\mathrm{CH}_{2} \mathrm{Br}_{2}$ an internal standard. Cy-Xantphos = (9,9-dimethyl-4a,9a-dihydro-9H-xanthene-4,5-diyl)bis(dicyclohexylphosphane).

\section{Synthesis of Starting Materials}

General Procedure A:

$$
\mathrm{R}_{1}-\mathrm{CHO} \quad \frac{\mathrm{R}_{2}-\mathrm{MgX}}{\mathrm{Et}_{2} \mathrm{O}, 0^{\circ} \mathrm{C}-35^{\circ} \mathrm{C}}
$$

To a solution of alkenyl or aryl aldehyde (1.0 equiv.) in $\mathrm{Et}_{2} \mathrm{O}$ was dropwise added Grignard (1.5 equiv.) at $0{ }^{\circ} \mathrm{C}$. The resulting mixture was then heated to $35{ }^{\circ} \mathrm{C}$ in an oil bath and stirred for $3 \mathrm{~h}$. Upon completion, the reaction was slowly quenched with saturated $\mathrm{NH}_{4} \mathrm{Cl}$ solution at $0{ }^{\circ} \mathrm{C}$, and extracted with EtOAc. The organic layer was dried with $\mathrm{Na}_{2} \mathrm{SO}_{4}$, filtered, and concentrated. The residue was purified by silica gel flash chromatography (petroleum ether: EtOAc) to afford the alkenyl alcohol. 
General Procedure B:

$$
\mathrm{Ar}-\mathrm{Br} \quad \frac{\text { 1) } n-\mathrm{BuLi}, \mathrm{THF},-78^{\circ} \mathrm{C}}{\text { 2) } \mathrm{R}-\mathrm{CHO}, \mathrm{rt}}
$$

To a solution of aryl bromide (1.0 equiv) in THF was added $n$-BuLi (1.1 equiv) at $-78^{\circ} \mathrm{C}$. After stirring for $30 \mathrm{~min}$, alkenyl aldehyde (1.2 equiv) was added. The resulting mixture was warmed up to room temperature and stirred for $6 \mathrm{~h}$. The reaction was slowly quenched with saturated $\mathrm{NH}_{4} \mathrm{Cl}$, and extracted with EtOAc. The organic layer was dried with $\mathrm{Na}_{2} \mathrm{SO}_{4}$, filtered, and concentrated. The residue was purified by silica gel flash chromatography (petroleum ether: EtOAc) to afford the alkenyl alcohol.

\section{1-Phenylundec-10-en-1-ol (1a).}

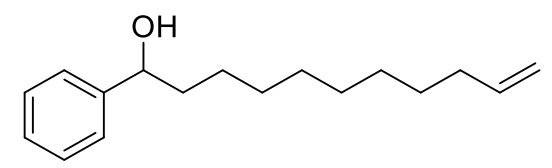

Compound 1a was prepared as a colorless oil in $82 \%$ yield (1.00 g, eluent: petroleum ether: $\mathrm{EtOAc}=30: 1)$ from undec-10-enal $(0.84 \mathrm{~g}, 5.00 \mathrm{mmol})$ and phenylmagnesium bromide $\left(2.5 \mathrm{~mL}, 3.0 \mathrm{M}\right.$ in $\left.\mathrm{Et}_{2} \mathrm{O}\right)$ following the above general procedure A. This compound was previously reported. ${ }^{3}$

$\mathbf{R}_{f}=0.37$ (petroleum ether: EtOAc $=10: 1$ )

${ }^{1} \mathrm{H}$ NMR (400 MHz, $\left.\mathrm{CDCl}_{3}\right)$ ठ 7.38-7.24 (m, 5H), 5.89-5.72 (m, 1H), 5.05-4.86 (m, $2 \mathrm{H}), 4.74-4.54(\mathrm{~m}, 1 \mathrm{H}), 2.07-1.97(\mathrm{~m}, 2 \mathrm{H}), 1.94(\mathrm{~s}, 1 \mathrm{H}), 1.82-1.64(\mathrm{~m}, 2 \mathrm{H}), 1.41-$ $1.20(\mathrm{~m}, 12 \mathrm{H})$.

${ }^{13} \mathrm{C}$ NMR (100 MHz, $\left.\mathrm{CDCl}_{3}\right) \delta$ 145.1, 139.3, 128.5, 127.5, 126.0, 114.2, 74.7, 39.2, $33.9,29.61,29.59,29.5,29.2,29.0,25.9$.

\section{1-Phenylbut-3-en-1-ol (1b).}<smiles>C=CCC(O)c1ccccc1</smiles> 
Compound $\mathbf{~} \mathbf{b}$ was prepared as a colorless oil in $85 \%$ yield $(0.63 \mathrm{~g}$, eluent: petroleum ether: EtOAc $=30: 1)$ from benzaldehyde $(0.53 \mathrm{~g}, 5.00 \mathrm{mmol})$, allylmagnesium bromide $(7.5 \mathrm{~mL}, 1.0 \mathrm{M}$ in THF) following the above general procedure A. This compound was previously reported. ${ }^{4}$

$\mathbf{R}_{f}=0.37$ (petroleum ether: EtOAc $=10: 1$ )

${ }^{1} \mathrm{H}$ NMR (400 MHz, $\left.\mathrm{CDCl}_{3}\right)$ : $\delta$ 7.37-7.27 (m, 5H), 5.89-5.75 (m, 1H), 5.20-5.13 (m, 2H), 4.81-4.70 (m, 1H), 2.54-2.49 (m, 2H), 2.31-2.10 (m, 1H).

${ }^{13} \mathrm{C}$ NMR (100 MHz, $\mathrm{CDCl}_{3}$ ): $\delta$ 143.9, 134.5, 128.4, 127.6, 125.8, 118.4, 73.3, 43.8

\section{1-Phenylhex-5-en-1-ol (1c).}

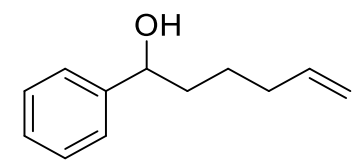

Compound 1c was prepared as a colorless oil in $76 \%$ yield $(0.27 \mathrm{~g}$, eluent: petroleum ether: $\mathrm{EtOAc}=30: 1)$ from hex-5-enal ${ }^{5}(0.20 \mathrm{~g}, 2.0 \mathrm{mmol})$,

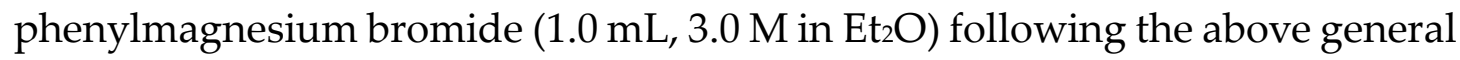
procedure A. This compound was previously reported. ${ }^{6}$

$\mathbf{R}_{f}=0.37$ (petroleum ether: EtOAc $=10: 1$ )

${ }^{1} \mathrm{H}$ NMR (400 MHz, CDCl $\left.)_{3}\right)$ 8 7.33-7.21 (m, 5H), 5.83-5.70 (m, 1H), 5.00-4.90 (m, $2 \mathrm{H}), 4.60-4.53(\mathrm{~m}, 1 \mathrm{H}), 2.59-2.51(\mathrm{~m}, 1 \mathrm{H}), 1.32-2.07(\mathrm{~m}, 6 \mathrm{H})$.

${ }^{13} \mathrm{C}$ NMR (100 MHz, $\left.\mathrm{CDCl}_{3}\right) \delta$ 144.9, 138.7, 128.5, 127.5, 126.0, 114.7, 74.5, 38.5, 33.6, 25.1.

2-Methyl-1-phenylprop-2-en-1-ol (1d).<smiles>C=C(C)C(O)c1ccccc1</smiles>

Compound 1d was prepared as a colorless oil in $42 \%$ yield $(0.31 \mathrm{~g}$, eluent: petroleum ether: $\mathrm{EtOAc}=30: 1)$ from methacrylaldehyde $(0.35 \mathrm{~g}, 5.00 \mathrm{mmol})$, 


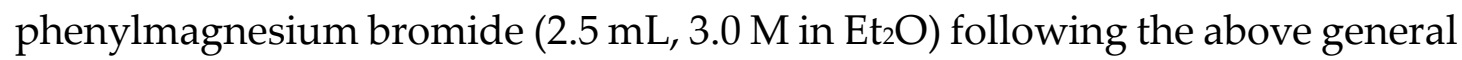
procedure A. This compound was previously reported. ${ }^{7}$

$\mathbf{R}_{f}=0.37$ (petroleum ether: EtOAc $=10: 1$ )

${ }^{1} \mathrm{H}$ NMR (400 MHz, CDCl $)$ \& 7.46-7.19 (m, 5H), 5.81-5.53 (m, 2H), $5.08(\mathrm{~s}, 1 \mathrm{H})$, 2.75-2.32 (m, 1H), 1.81-1.62 (m, 3H).

${ }^{13} \mathrm{C}$ NMR (100 MHz, $\left.\mathrm{CDCl}_{3}\right) \delta$ 143.4, 133.7, 129.5, 128.5, 127.5, 126.2, 75.2, 17.8.

(E)-1-Phenylbut-2-en-1-ol (1 $\left.b^{\prime}\right)$.<smiles>C/C=C/C(O)c1ccccc1</smiles>

Compound $\mathbf{1} \mathbf{b}^{\prime}$ was prepared as a colorless oil in $49 \%$ yield ( $0.36 \mathrm{~g}$, eluent: petroleum ether: EtOAc $=30: 1)$ from $(E)$-but-2-enal $(0.35 \mathrm{~g}, 5.00 \mathrm{mmol})$ and

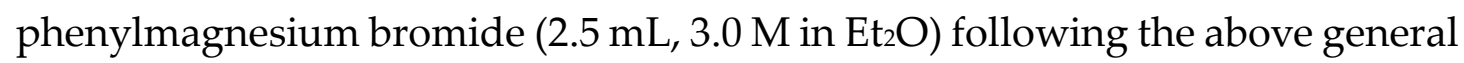
procedure A. This compound was previously reported. ${ }^{8}$

$\mathbf{R}_{f}=0.37$ (petroleum ether: EtOAc $=10: 1$ )

${ }^{1} \mathrm{H}$ NMR (400 MHz, $\left.\mathrm{CDCl}_{3}\right)$ \& 7.42-7.18 (m, 5H), 5.80-5.57 (m, 2H), 5.08 (d, $J=$ $6.1 \mathrm{~Hz}, 1 \mathrm{H}), 2.88-2.28(\mathrm{~m}, 1 \mathrm{H}), 1.68(\mathrm{~d}, J=5.7 \mathrm{~Hz}, 3 \mathrm{H})$.

${ }^{13} \mathrm{C}$ NMR (100 MHz, $\mathrm{CDCl}_{3}$ ) $\delta$ 143.4, 133.7, 129.5, 128.5, 127.5, 126.2, 75.2, 17.8.

(E)-1-Phenylhex-4-en-1-ol (1c').

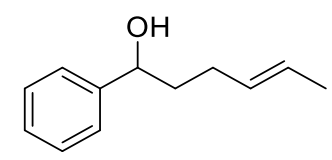

Compound $1 \mathbf{c}^{\prime}$ was prepared as a colorless oil in $36 \%$ yield $(0.32 \mathrm{~g}$, eluent: petroleum ether: EtOAc $=30: 1)$ from $(E)$-hex-4-enal $(0.49 \mathrm{~g}, 5.00 \mathrm{mmol})$ and

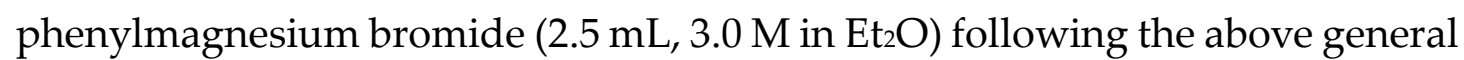
procedure A. This compound was previously reported. ${ }^{9}$

$\mathbf{R}_{f}=0.37$ (petroleum ether: EtOAc $=10: 1$ )

${ }^{1} \mathrm{H}$ NMR $\left(400 \mathrm{MHz}, \mathrm{CDCl}_{3}\right)$ \& 7.51-7.21 (m, 5H), 5.59-5.43 (m, 2H), $4.69(\mathrm{t}, J=$ $6.7 \mathrm{~Hz}, 1 \mathrm{H}), 2.46(\mathrm{~s}, 1 \mathrm{H}), 2.22-2.00(\mathrm{~m}, 2 \mathrm{H}), 1.98-1.75(\mathrm{~m}, 2 \mathrm{H}), 1.75-1.69(\mathrm{~m}, 3 \mathrm{H})$. 
${ }^{13} \mathrm{C}$ NMR (100 MHz, $\left.\mathrm{CDCl}_{3}\right) \delta$ 144.8, 130.7, 128.4, 127.5, 126.0, 125.5, 74.0, 38.8, $28.9,18.0$.

(E)-1-Phenylhex-2-en-1-ol (1c').

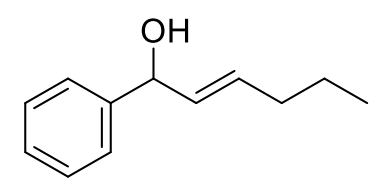

Compound $1 \mathbf{c}^{\prime \prime}$ was prepared as a colorless oil in 35\% yield ( $0.31 \mathrm{~g}$, eluent: petroleum ether: $\mathrm{EtOAc}=30: 1)$ from $(E)$-hex-2-enal $(0.49 \mathrm{~g}, 5.00 \mathrm{mmol})$ and phenylmagnesium bromide $\left(2.5 \mathrm{~mL}, 3.0 \mathrm{M}\right.$ in $\left.\mathrm{Et}_{2} \mathrm{O}\right)$ following the above general procedure A.

$\mathbf{R}_{f}=0.37$ (petroleum ether: $\mathrm{EtOAc}=10: 1$ )

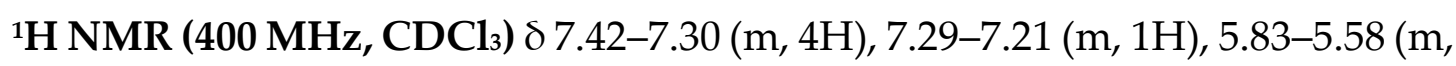
2H), 5.18-5.10 (m, 1H), 2.10-1.98 (m, 3H), 1.48-1.34 (m, 2H), 0.94-0.85 (m, 3H). ${ }^{13} \mathrm{C}$ NMR (100 MHz, $\left.\mathrm{CDCl}_{3}\right) \delta$ 143.5, 132.6, 132.5, 128.5, 127.5, 126.3, 75.3, 34.4, $22.3,13.8$.

HRMS (ESI') calcd for $\mathrm{C}_{12} \mathrm{H}_{16} \mathrm{NaO}^{+}[\mathrm{M}+\mathrm{Na}]^{+}$: 199.1093, found: 199.1093.

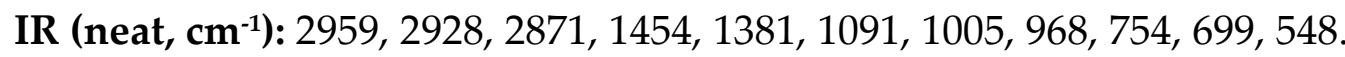

\section{(Z)-1-Phenyldec-5-en-1-ol (1e).}

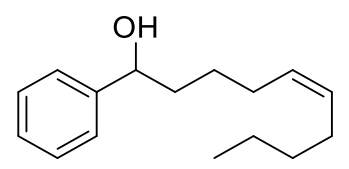

Compound 1e was prepared as a colorless oil in $86 \%$ yield $(0.40 \mathrm{~g}$, eluent: petroleum ether: $\mathrm{EtOAc}=30: 1)$ from $(Z)$-dec-5-enal $(0.38 \mathrm{~g}, 2.00 \mathrm{mmol})$ and phenylmagnesium bromide $\left(1.0 \mathrm{~mL}, 3.0 \mathrm{M}\right.$ in $\left.\mathrm{Et}_{2} \mathrm{O}\right)$ following the above general procedure A.

$\mathbf{R}_{f}=0.37$ (petroleum ether: $\mathrm{EtOAc}=10: 1$ )

${ }^{1} \mathrm{H}$ NMR (400 MHz, $\left.\mathrm{CDCl}_{3}\right) \delta$ 7.45-7.17 (m, 5H), 5.52-5.29 (m, 2H), 4.69-4.53 (m, $1 \mathrm{H}), 2.27-2.22(\mathrm{~m}, 1 \mathrm{H}), 2.15-2.02(\mathrm{~m}, 2 \mathrm{H}), 2.02-1.93(\mathrm{~m}, 2 \mathrm{H}), 1.88-1.66(\mathrm{~m}, 2 \mathrm{H})$, $1.37-1.22(\mathrm{~m}, 6 \mathrm{H}), 0.88(\mathrm{t}, J=6.8 \mathrm{~Hz}, 3 \mathrm{H})$. 
${ }^{13} \mathrm{C}$ NMR (100 MHz, $\left.\mathrm{CDCl}_{3}\right) \delta$ 144.8, 130.9, 128.9, 128.5, 127.5, 126.0, 74.2, 39.0, $31.6,29.5,27.3,23.7,22.7,14.2$.

HRMS (ESI') calcd for $\mathrm{C}_{16} \mathrm{H}_{24} \mathrm{NaO}^{+}[\mathrm{M}+\mathrm{Na}]^{+}:$255.1719, found: 255. 1719 .

IR (neat, cm-1): 2956, 2925, 2857, 1454, 1061, 1022, 759, 700.

(E)-1-Phenylbut-2-en-1-ol (1f).<smiles>OC(c1ccccc1)C1CC=CCC1</smiles>

Compound 1f was prepared as a white solid in 55\% yield (0.52 g, eluent: petroleum ether: $\mathrm{EtOAc}=30: 1, \mathrm{dr}=5: 4)$ from cyclohex-3-ene-1-carbaldehyde $(0.55 \mathrm{~g}, 5.00 \mathrm{mmol})$ and phenylmagnesium bromide $\left(2.5 \mathrm{~mL}, 3.0 \mathrm{M}\right.$ in $\left.\mathrm{Et}_{2} \mathrm{O}\right)$ following the above general procedure A.

$\mathbf{R}_{f}=0.37$ (petroleum ether: $\mathrm{EtOAc}=10: 1$ )

${ }^{1} \mathrm{H}$ NMR (400 MHz, $\left.\mathrm{CDCl}_{3}\right)$ ठ 7.39-7.20 (m, 5H), 5.76-5.53 (m, 2H), 4.49-4.32 (m, $1 \mathrm{H}), 2.39-1.78(\mathrm{~m}, 6 \mathrm{H}), 1.69-1.47(\mathrm{~m}, 1 \mathrm{H}), 1.43-1.13(\mathrm{~m}, 1 \mathrm{H})$.

${ }^{13} \mathrm{C}$ NMR (100 MHz, $\left.\mathrm{CDCl}_{3}\right) \delta$ 143.6, 143.5, 128.4, 128.4, 127.7, 127.7, 127.2, 126.9, 126.8, 126.7, 126.3, 126.1, 78.9, 78.6, 41.1, 41.0, 28.3, 27.6, 25.3, 25.2, 25.1, 24.9.

HRMS (ESI') calcd for $\mathrm{C}_{13} \mathrm{H}_{16} \mathrm{NaO}^{+}[\mathrm{M}+\mathrm{Na}]^{+}$: 211.1093, found: 211.1093.

IR (neat, cm-1): 3023, 2913, 2838, 1452, 1015, 765, 734, 701, 655, 554.

Bicyclo[2.2.1]hept-5-en-2-yl(phenyl)methanol (1g).<smiles>OC(c1ccccc1)C1CC2C=CC1C2</smiles>

Compound $1 \mathrm{~g}$ was prepared as a colorless oil in 55\% yield ( $0.55 \mathrm{~g}$, eluent: petroleum ether: $\mathrm{EtOAc}=30: 1, \mathrm{dr}=1: 2.6: 3.7: 25.6)$ from bicyclo[2.2.1]hept-5ene-2-carbaldehyde $(0.61 \mathrm{~g}, 5.00 \mathrm{mmol})$ and phenylmagnesium bromide (2.5 $\mathrm{mL}$, 3.0 $\mathrm{M}$ in $\mathrm{Et}_{2} \mathrm{O}$ ) following the above general procedure A. This compound was previously reported. ${ }^{10}$

$\mathbf{R}_{f}=0.37$ (petroleum ether: $\mathrm{EtOAc}=10: 1$ ) 
${ }^{1} \mathrm{H}$ NMR (400 MHz, $\mathrm{CDCl}_{3}$ ) 8 7.42-7.17 (m, 5H), 6.31-5.92 (m, 2H), 4.48-3.90 (m, $1 \mathrm{H}), 3.24-3.05(\mathrm{~m}, 1 \mathrm{H}), 2.93-2.70(\mathrm{~m}, 1 \mathrm{H}), 2.55-1.91(\mathrm{~m}, 1 \mathrm{H}), 1.68-1.40(\mathrm{~m}, 2 \mathrm{H})$, $1.36-0.81(\mathrm{~m}, 2 \mathrm{H}), 0.54-0.43(\mathrm{~m}, 1 \mathrm{H})$.

${ }^{13} \mathrm{C}$ NMR (100 MHz, $\left.\mathrm{CDCl}_{3}\right) \delta$ 144.3, 138.1, 132.8, 128.5, 127.8, 127.1, 78.6, 49.5, $46.9,44.4,42.3,29.5$.

1-(4-Methylphenyl)undec-10-en-1-ol (1h).

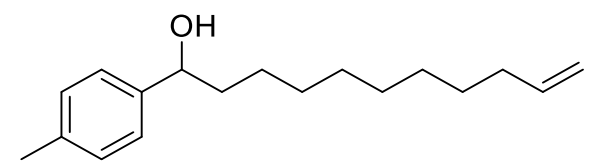

Compound $1 \mathrm{~h}$ was prepared as a colorless oil in $50 \%$ yield $(0.66 \mathrm{~g}$, eluent: petroleum ether: EtOAc $=30: 1)$ from undec-10-enal $(0.84 \mathrm{~g}, 5.00 \mathrm{mmol})$ and 4methylphenylmagnesium bromide $(7.5 \mathrm{~mL}, 1.0 \mathrm{M}$ in THF) following the above general procedure $\mathrm{A}$.

$\mathbf{R}_{f}=0.38$ (petroleum ether: $\mathrm{EtOAc}=10: 1$ )

${ }^{1} \mathrm{H}$ NMR (400 MHz, CDCl$\left.)_{3}\right) \delta 7.23(\mathrm{~d}, J=8.1 \mathrm{~Hz}, 2 \mathrm{H}), 7.16(\mathrm{~d}, J=7.9 \mathrm{~Hz}, 2 \mathrm{H})$, 5.95-5.65 (m, 1H), 5.07-4.86 (m, 2H), 4.75-4.49 (m, 1H), $2.34(\mathrm{~s}, 3 \mathrm{H}), 2.08-1.97$ (m, 2H), 1.85-1.73 (m, 2H), 1.73-1.63 (m, 1H), 1.45-1.19 (m, 12H).

${ }^{13} \mathrm{C}$ NMR (100 MHz, $\mathrm{CDCl}_{3}$ ) ठ 142.1, 139.4, 137.2, 129.2, 126.0, 114.2, 74.7, 39.2, 33.9, 29.7, 29.6, 29.5, 29.2, 29.0, 26.0, 21.3.

HRMS (ESI') calcd for $\mathrm{C}_{18} \mathrm{H}_{28} \mathrm{NaO}^{+}$[M+Na] $]^{+}$283.2032, found: 283.2032 .

IR (neat, cm$^{-1}$ ): 2925, 2855, 1641, 1514, 1460, 995, 909, 817, 723, 638, 552.

1-(3,5-Dimethylphenyl)undec-10-en-1-ol (1i).

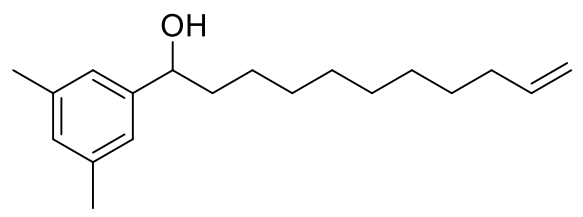

Compound $1 \mathrm{i}$ was prepared as a white solid in $88 \%$ yield $(1.21 \mathrm{~g}$, eluent: petroleum ether: $\mathrm{EtOAc}=30: 1)$ from 1-bromo-3,5-dimethylbenzene ( $0.92 \mathrm{~g}, 5.00$ $\mathrm{mmol}), n$-BuLi (6.9 mL, 1.6 M in hexane), and undec-10-enal (1.00 g, $6.00 \mathrm{mmol})$ following the above general procedure $\mathrm{B}$. 
$\mathbf{R}_{f}=0.35$ (petroleum ether: $\mathrm{EtOAc}=10: 1$ )

${ }^{1} \mathrm{H}$ NMR (400 MHz, $\left.\mathrm{CDCl}_{3}\right) \delta$ 7.08-6.79 (m, 3H), 5.87-5.73 (m, 1H), 5.21-4.79 (m, $2 \mathrm{H}), 4.71-4.45(\mathrm{~m}, 1 \mathrm{H}), 2.30$ (s, 6H), 2.08-1.96 (m, 3H), 1.81-1.58 (m, 2H), $1.41-$ $1.22(\mathrm{~m}, 12 \mathrm{H})$.

${ }^{13} \mathrm{C}$ NMR (100 MHz, $\left.\mathrm{CDCl}_{3}\right) \delta$ 145.1, 139.3, 138.0, 129.1, 123.8, 114.2, 74.8, 39.1, $33.9,29.64,29.59,29.5,29.2,29.0,26.0,21.4$.

HRMS (ESI ${ }^{+}$) calcd for $\mathrm{C}_{19} \mathrm{H}_{30} \mathrm{NaO}^{+}[\mathrm{M}+\mathrm{Na}]^{+}:$297.2189, found: 297.2188.

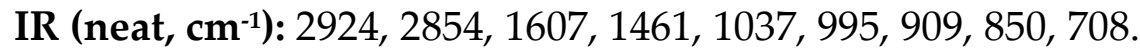

1-(4-Methoxyphenyl)undec-10-en-1-ol (1j).

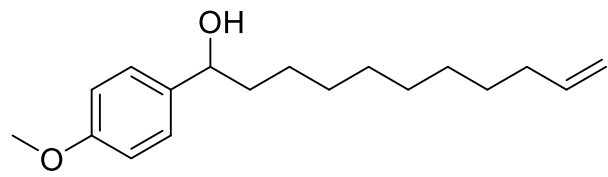

Compound $1 \mathbf{j}$ was prepared as a colorless oil in 57\% yield $(0.79 \mathrm{~g}$, eluent: petroleum ether: $\mathrm{EtOAc}=10: 1)$ from undec-10-enal $(0.84 \mathrm{~g}, 5.00 \mathrm{mmol})$ and 4methoxyphenylmagnesium bromide $(7.5 \mathrm{~mL}, 1.0 \mathrm{M}$ in THF) following the above general procedure $\mathrm{A}$.

$\mathbf{R}_{f}=0.12$ (petroleum ether: $\mathrm{EtOAc}=10: 1$ )

${ }^{1}$ H NMR (400 MHz, $\left.\mathrm{CDCl}_{3}\right) \delta 7.26(\mathrm{~d}, J=8.7 \mathrm{~Hz}, 2 \mathrm{H}), 6.88(\mathrm{~d}, J=8.6 \mathrm{~Hz}, 2 \mathrm{H})$, 5.93-5.68 (m, 1H), 5.07-4.88 (m, 2H), 4.69-4.55 (m, 1H), 3.80 (s, 3H), 2.09-1.99 (m, 2H), 1.85-1.72 (m, 2H), 1.71-1.61 (m, 1H), 1.37-1.21 (m, 12H).

${ }^{13} \mathrm{C}$ NMR (100 MHz, $\left.\mathrm{CDCl}_{3}\right) \delta$ 158.9, 139.2, 137.2, 127.2, 114.2, 113.8, 74.2, 55.3, $39.0,33.9,29.59,29.57,29.5,29.2,29.0,25.9$.

HRMS (ESI') calcd for $\mathrm{C}_{18} \mathrm{H}_{28} \mathrm{NaO}_{2}{ }^{+}[\mathrm{M}+\mathrm{Na}]^{+}:$299.1982, found: 299.1981.

IR (neat, cm-1): 2924, 2854, 1512, 1461, 1301, 1245, 1176, 1037, 996, 909, 831, 556.

1-(3,4-Dimethoxyphenyl)undec-10-en-1-ol (1k).<smiles>C=CCCCCCCCCC(O)c1ccc(OC)c(OC)c1</smiles> 
Compound 1k was prepared as a white solid in 76\% yield (1.16 g, eluent: petroleum ether: $\mathrm{EtOAc}=2: 1)$ from 4-bromo-1,2-dimethoxybenzene (1.08 g, $5.00 \mathrm{mmol}), n$-BuLi (6.9 mL, 1.6 M in hexane), and undec-10-enal (1.00 g, 6.00 mmol) following the above general procedure $B$.

$\mathbf{R}_{f}=0.14$ (petroleum ether: $\mathrm{EtOAc}=2: 1$ )

${ }^{1} \mathrm{H}$ NMR (400 MHz, $\left.\mathrm{CDCl}_{3}\right) \delta 6.90(\mathrm{~s}, 1 \mathrm{H}), 6.87-6.79(\mathrm{~m}, 2 \mathrm{H}), 5.90-5.68(\mathrm{~m}, 1 \mathrm{H})$, 5.07-4.87 (m, 2H), 4.64-4.51 (m, 1H), 3.93-3.83 (m, 6H), 2.10-1.95 (m, 2H), 1.89$1.74(\mathrm{~m}, 2 \mathrm{H}), 1.71-1.64(\mathrm{~m}, 1 \mathrm{H}), 1.42-1.22(\mathrm{~m}, 12 \mathrm{H})$.

${ }^{13} \mathrm{C}$ NMR (100 MHz, $\left.\mathrm{CDCl}_{3}\right) \delta$ 149.2, 148.5, 139.3, 137.8, 118.3, 114.2, 111.0, 109.1, $74.7,56.04,55.97,39.2,33.9,29.6,29.5,29.2,29.0,26.0$.

HRMS (ESI ${ }^{+}$) calcd for $\mathrm{C}_{19} \mathrm{H}_{30} \mathrm{NaO}_{3}{ }^{+}[\mathrm{M}+\mathrm{Na}]^{+}: 329.2087$, found: 329.2087.

IR (neat, cm ${ }^{-1}$ ): 2926, 2854, 1515, 1461, 1418, 1262, 1235, 1141, 1030, 909, 808, 764.

\section{1-(4-Fluorophenyl)undec-10-en-1-ol (11).}

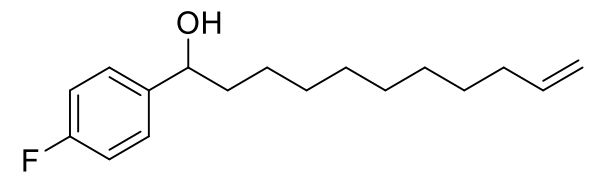

Compound 11 was prepared as a colorless oil in $66 \%$ yield $(0.87 \mathrm{~g}$, eluent: petroleum ether: $\mathrm{EtOAc}=30: 1)$ from 1-bromo-4-fluorobenzene $(0.87 \mathrm{~g}, 5.00$ $\mathrm{mmol}), n$-BuLi (6.9 mL, 1.6 M in hexane), and undec-10-enal (1.00 g, $6.00 \mathrm{mmol})$ following the above general procedure B.

$\mathbf{R}_{f}=0.38$ (petroleum ether: $\mathrm{EtOAc}=10: 1$ )

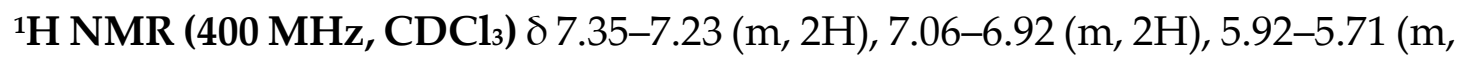
$1 \mathrm{H}), 5.05-4.86(\mathrm{~m}, 2 \mathrm{H}), 4.65-4.52(\mathrm{~m}, 1 \mathrm{H}), 2.20$ (s, 1H), 2.09-1.97 (m, 2H), 1.79$1.58(\mathrm{~m}, 2 \mathrm{H}), 1.42-1.18(\mathrm{~m}, 12 \mathrm{H})$.

${ }^{13} \mathrm{C}$ NMR (100 MHz, $\left.\mathrm{CDCl}_{3}\right) \delta 162.2(\mathrm{~d}, J=245.1 \mathrm{~Hz}), 140.7(\mathrm{~d}, J=3.1 \mathrm{~Hz}), 139.3$, $127.6(\mathrm{~d}, J=8.0 \mathrm{~Hz}), 115.3(\mathrm{~d}, J=21.3 \mathrm{~Hz}), 114.2,74.1,39.3,33.9$, 29.6 (double), $29.5,29.2,29.0,25.9$.

HRMS (ESI ${ }^{+}$) calcd for $\mathrm{C}_{17} \mathrm{H}_{25} \mathrm{FNaO}^{+}[\mathrm{M}+\mathrm{Na}]^{+}: 287.1782$, found: 287.1782 .

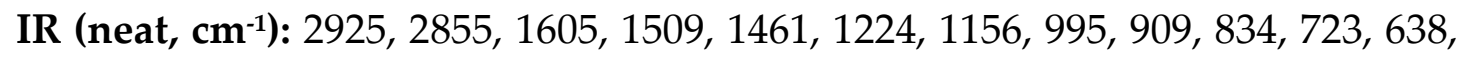
$573,551$.

\section{1-(4-Chlorophenyl)undec-10-en-1-ol (1m).}




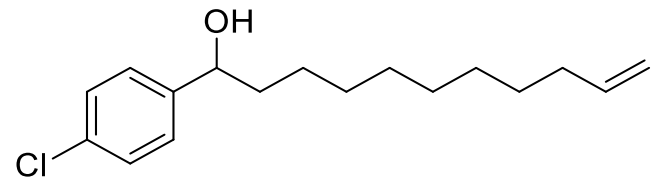

Compound $1 \mathrm{~m}$ was prepared as a colorless oil in $76 \%$ yield (1.06 g, eluent: petroleum ether: EtOAc $=30: 1)$ from 1-bromo-4-chlorobenzene $(0.95 \mathrm{~g}, 5.00$ $\mathrm{mmol}), n$-BuLi (6.9 mL, 1.6 M in hexane), and undec-10-enal (1.00 g, $6.00 \mathrm{mmol})$ following the above general procedure $\mathrm{B}$.

$\mathbf{R}_{f}=0.30$ (petroleum ether: $\mathrm{EtOAc}=10: 1$ )

${ }^{1} \mathrm{H}$ NMR (400 MHz, $\left.\mathrm{CDCl}_{3}\right)$ \& 7.34-7.14 (m, 4H), 5.97-5.65 (m, 1H), 5.10-4.82 (m, $2 \mathrm{H}), 4.69-4.43(\mathrm{~m}, 1 \mathrm{H}), 2.16(\mathrm{~s}, 1 \mathrm{H}), 2.09-1.98(\mathrm{~m}, 2 \mathrm{H}), 1.81-1.57(\mathrm{~m}, 2 \mathrm{H}), 1.38-$ $1.20(\mathrm{~m}, 12 \mathrm{H})$.

${ }^{13} \mathrm{C}$ NMR (100 MHz, $\left.\mathrm{CDCl}_{3}\right) \delta$ 143.5, 139.3, 133.1, 128.6, 127.4, 114.2, 74.0, 39.2, 33.9, 29.6 (double), 29.5, 29.2, 29.0, 25.8 .

HRMS (ESI') calcd for $\mathrm{C}_{17} \mathrm{H}_{25} \mathrm{ClNaO}^{+}[\mathrm{M}+\mathrm{Na}]^{+}: 303.1486$, found: 303.1486 .

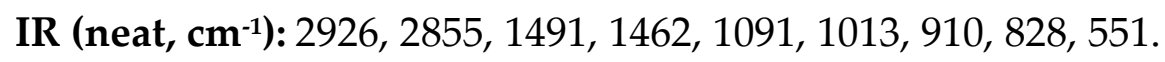

1-(4-(Trifluoromethyl)phenyl)undec-10-en-1-ol (1n).

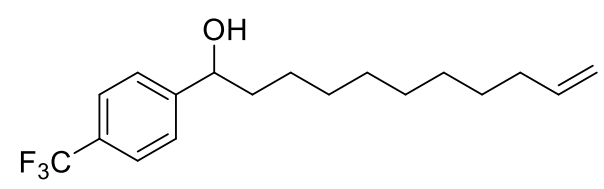

Compound 1n was prepared as a colorless oil in 65\% yield (1.02 g, eluent: petroleum ether: $\mathrm{EtOAc}=30: 1)$ from 1-bromo-4-(trifluoromethyl)benzene (1.16 g, $5.00 \mathrm{mmol}), n$-BuLi (6.9 mL, 1.6 M in hexane), and undec-10-enal (1.00 g, 6.00 $\mathrm{mmol}$ ) following the above general procedure $\mathrm{B}$.

$\mathbf{R}_{f}=0.25$ (petroleum ether: $\left.\mathrm{EtOAc}=10: 1\right)$

${ }^{1} \mathrm{H}$ NMR (400 MHz, $\left.\mathrm{CDCl}_{3}\right) \delta 7.59(\mathrm{~d}, J=8.0 \mathrm{~Hz}, 2 \mathrm{H}), 7.44(\mathrm{~d}, J=7.9 \mathrm{~Hz}, 2 \mathrm{H})$, 5.93-5.69 (m, 1H), 5.07-4.85 (m, 2H), 4.77-4.65 (m, 1H), 2.26-2.09 (m, 1H), 2.10$1.96(\mathrm{~m}, 2 \mathrm{H}), 1.86-1.63(\mathrm{~m}, 2 \mathrm{H}), 1.46-1.21(\mathrm{~m}, 12 \mathrm{H})$.

${ }^{13} \mathrm{C}$ NMR (100 MHz, CDCl$)$ ) $149.0,139.3,129.7$ (q, J = 32.2 Hz), 126.3, 125.5 (q, $J=3.6 \mathrm{~Hz}$ ), $124.3(\mathrm{q}, J=270 \mathrm{~Hz}), 114.3,74.1,39.4,33.9,29.6$ (double), 29.5, 29.2, 29.0, 25.7.

HRMS (ESI ${ }^{+}$) calcd for $\mathrm{C}_{18} \mathrm{H}_{25} \mathrm{~F}_{3} \mathrm{NaO}^{+}[\mathrm{M}+\mathrm{Na}]^{+}$: 337.1750, found: 337.1749 . 
IR (neat, cm $\left.{ }^{-1}\right)$ : 2927, 2856, 1417, 1324, 1164, 1125, 1067, 1016, 910, 841, 606.

1-(6-((tert-Butyldimethylsilyl)oxy)naphthalen-2-yl)undec-10-en-1-ol (10).

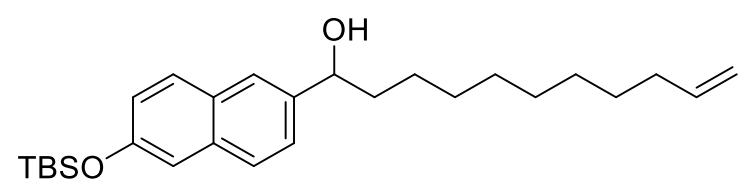

Compound 1o was prepared as a white solid in 68\% yield (1.14 g, eluent: petroleum ether: EtOAc = 30:1) from ((6-bromonaphthalen-2-yl)oxy)(tertbutyl)dimethylsilane (1.68 g, $5.00 \mathrm{mmol}), n$-BuLi (6.9 mL, 1.6 $\mathrm{M}$ in hexane), and undec-10-enal $(1.00 \mathrm{~g}, 6.00 \mathrm{mmol})$ following the above general procedure B.

$\mathbf{R}_{f}=0.30$ (petroleum ether: $\mathrm{EtOAc}=10: 1$ )

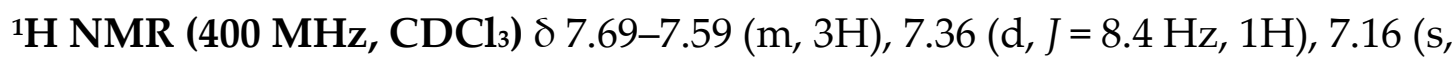
$1 \mathrm{H}), 7.05(\mathrm{~d}, J=8.5 \mathrm{~Hz}, 1 \mathrm{H}), 5.86-5.71(\mathrm{~m}, 1 \mathrm{H}), 5.04-4.87(\mathrm{~m}, 2 \mathrm{H}), 4.74-4.64(\mathrm{~m}$, $1 \mathrm{H}), 2.37-2.23(\mathrm{~m}, 1 \mathrm{H}), 2.08-1.96(\mathrm{~m}, 2 \mathrm{H}), 1.88-1.66(\mathrm{~m}, 2 \mathrm{H}), 1.43-1.20(\mathrm{~m}, 13 \mathrm{H})$, $1.02(\mathrm{~s}, 9 \mathrm{H}), 0.24(\mathrm{~s}, 6 \mathrm{H})$.

${ }^{13} \mathrm{C}$ NMR (100 MHz, $\left.\mathrm{CDCl}_{3}\right) \delta$ 153.5, 140.3, 139.3, 134.2, 129.4, 129.1, 127.1, 124.6, $124.5,122.3,114.9,114.2,74.8,39.0,33.9,29.7,29.6,29.5,29.2,29.0,26.0,25.8,18.4$, -4.2 .

HRMS (ESI ${ }^{+}$) calcd for $\mathrm{C}_{27} \mathrm{H}_{42} \mathrm{NaO}_{2} \mathrm{Si}^{+}[\mathrm{M}+\mathrm{Na}]^{+}:$449.2846, found: 449.2845 .

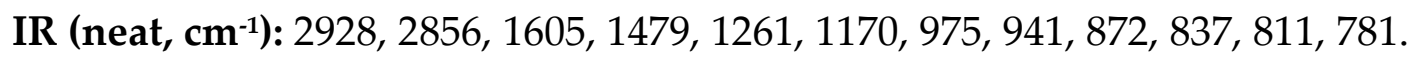

1-(2-Methoxynaphthalen-1-yl)undec-10-en-1-ol (1p).

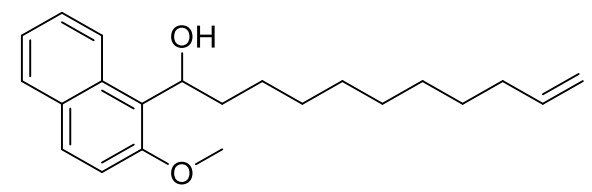

Compound 1p was prepared as a colorless oil in 54\% yield (0.88 g, eluent: petroleum ether: $\mathrm{EtOAc}=10: 1)$ from 1-bromo-2-methoxynaphthalene $(1.18 \mathrm{~g}$, $5.00 \mathrm{mmol}), n$-BuLi (6.9 mL, 1.6 M in hexane), and undec-10-enal (1.00 g, 6.00 $\mathrm{mmol}$ ) following the above general procedure $\mathrm{B}$.

$\mathbf{R}_{f}=0.16$ (petroleum ether: $\mathrm{EtOAc}=10: 1$ )

${ }^{1} \mathrm{H}$ NMR (400 MHz, $\left.\mathrm{CDCl}_{3}\right) \delta 8.09(\mathrm{~d}, J=8.7 \mathrm{~Hz}, 1 \mathrm{H}), 7.83-7.67$ (m, 2H), 7.52- 
$7.39(\mathrm{~m}, 1 \mathrm{H}), 7.36-7.28(\mathrm{~m}, 1 \mathrm{H}), 7.24(\mathrm{~d}, J=10.1 \mathrm{~Hz}, 1 \mathrm{H}), 5.92-5.67(\mathrm{~m}, 1 \mathrm{H}), 5.61-$ $5.47(\mathrm{~m}, 1 \mathrm{H}), 5.07-4.87(\mathrm{~m}, 2 \mathrm{H}), 3.95(\mathrm{~s}, 3 \mathrm{H}), 3.89-3.73(\mathrm{~m}, 1 \mathrm{H}), 2.05-1.97(\mathrm{~m}, 2 \mathrm{H})$, $1.94-1.82(\mathrm{~m}, 1 \mathrm{H}), 1.68-1.48(\mathrm{~m}, 1 \mathrm{H}), 1.43-1.20(\mathrm{~m}, 12 \mathrm{H})$.

${ }^{13} \mathrm{C}$ NMR (100 MHz, $\left.\mathrm{CDCl}_{3}\right) \delta$ o 154.5, 139.3, 131.8, 129.4, 129.2, 128.7, 126.6, 125.1, 123.6, 123.2, 114.2, 113.4, 70.0, 56.4, 37.8, 33.9, 29.7, 29.6, 29.5, 29.2, 29.0, 26.5.

HRMS (ESI+) calcd for $\mathrm{C}_{22} \mathrm{H}_{30} \mathrm{NaO}_{2}{ }^{+}[\mathrm{M}+\mathrm{Na}]^{+}: 349.2138$, found: 349.2137 .

IR (neat, cm-1): 2923, 2851, 1596, 1512, 1461, 1244, 1181, 1152, 1079, 1025, 907, 806,745 .

1-(Furan-2-yl)pent-4-en-1-ol (1q).

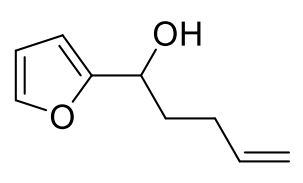

Compound $1 \mathbf{q}$ was prepared as a colorless oil in $15 \%$ yield $(0.11 \mathrm{~g}$, eluent: petroleum ether: EtOAc = 30:1) from furan-2-carbaldehyde $(0.48 \mathrm{~g}, 5.00 \mathrm{mmol})$ and but-3-en-1-ylmagnesium bromide (7.5 mL, $0.5 \mathrm{M}$ in THF) following the above general procedure A. This compound was previously reported. ${ }^{11}$

$\mathbf{R}_{f}=0.38$ (petroleum ether: $\mathrm{EtOAc}=10: 1$ )

${ }^{1} \mathrm{H}$ NMR (400 MHz, CDCl $)$ \& 7.39-7.31 (m, 1H), 6.36-6.29 (m, 1H), 6.26-6.18 (m, $1 \mathrm{H}), 5.91-5.73(\mathrm{~m}, 1 \mathrm{H}), 5.11-4.92(\mathrm{~m}, 2 \mathrm{H}), 4.73-4.61(\mathrm{~m}, 1 \mathrm{H}), 2.46-2.34(\mathrm{~m}, 1 \mathrm{H})$, $2.25-2.03(\mathrm{~m}, 2 \mathrm{H}), 1.97-1.87(\mathrm{~m}, 2 \mathrm{H})$.

${ }^{13} \mathrm{C}$ NMR (100 MHz, $\mathrm{CDCl}_{3}$ ) $\delta$ 156.7, 142.0, 137.9, 115.2, 110.2, 106.0, 67.2, 34.6, 29.8 .

1-(Thiophen-2-yl)pent-4-en-1-ol (1r).

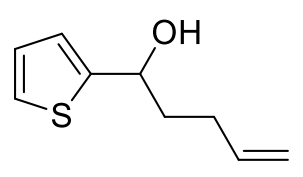

Compound $1 \mathbf{r}$ was prepared as a colorless oil in $11 \%$ yield $(0.09 \mathrm{~g}$, eluent: petroleum ether: EtOAc $=30: 1)$ from thiophene-2-carbaldehyde $(0.56 \mathrm{~g}, 5.00$ $\mathrm{mmol}$ ), and but-3-en-1-ylmagnesium bromide (7.5 mL, $0.5 \mathrm{M}$ in THF) following the above general procedure $\mathrm{A}$.

$\mathbf{R}_{f}=0.38$ (petroleum ether: $\mathrm{EtOAc}=10: 1$ ) 
${ }^{1} \mathrm{H}$ NMR (400 MHz, $\left.\mathrm{CDCl}_{3}\right)$ ठ 7.29-7.19 (m, 1H), 7.01-6.91 (m, 2H), 5.92-5.76 (m, 1H), 5.10-4.95 (m, 2H), 4.96-4.87 (m, 1H), 2.27 (s, 1H), 2.23-2.07 (m, 2H), 2.04$1.84(\mathrm{~m}, 2 \mathrm{H})$.

${ }^{13} \mathrm{C}$ NMR (100 MHz, $\left.\mathrm{CDCl}_{3}\right) \delta$ 148.7, 137.9, 126.7, 124.6, 123.8, 115.3, 69.8, 38.3, 30.1 .

1-(1-Methyl-1H-indol-5-yl)undec-10-en-1-ol (1s).

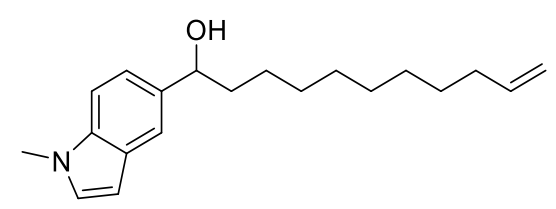

Compound 1s was prepared as a yellow oil in $76 \%$ yield $(1.13 \mathrm{~g}$, eluent: petroleum ether: $\mathrm{EtOAc}=20: 1)$ from 5-bromo-1-methyl- $1 H$-indole ${ }^{12}(1.00 \mathrm{~g}, 5.00$ $\mathrm{mmol}), n$-BuLi (6.9 mL, 1.6 M in hexane), and undec-10-enal (1.00 g, $6.00 \mathrm{mmol})$ following the above general procedure $\mathrm{B}$.

$\mathbf{R}_{f}=0.25$ (petroleum ether: $\mathrm{EtOAc}=10: 1$ )

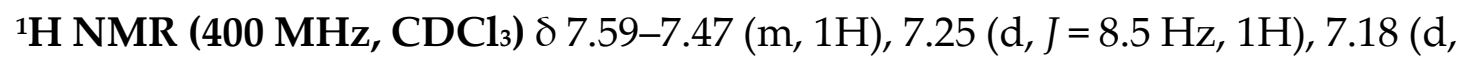
$J=8.3 \mathrm{~Hz}, 1 \mathrm{H}), 7.03-6.95(\mathrm{~m}, 1 \mathrm{H}), 6.46-6.40(\mathrm{~m}, 1 \mathrm{H}), 5.85-5.68(\mathrm{~m}, 1 \mathrm{H}), 5.05-$ $4.85(\mathrm{~m}, 2 \mathrm{H}), 4.72-4.63(\mathrm{~m}, 1 \mathrm{H}), 3.71(\mathrm{~s}, 3 \mathrm{H}), 2.13-1.97(\mathrm{~m}, 3 \mathrm{H}), 1.90-1.66(\mathrm{~m}, 2 \mathrm{H})$, $1.41-1.16(\mathrm{~m}, 12 \mathrm{H})$.

${ }^{13} \mathrm{C}$ NMR (100 MHz, $\left.\mathrm{CDCl}_{3}\right) \delta$ 139.3, 136.3, 136.0, 129.2, 128.3, 119.9, 118.4, 114.2, 109.2, 101.0, 75.4, 39.3, 33.9, 32.9, 29.65, 29.58, 29.5, 29.2, 29.0, 26.1.

HRMS (ESI ${ }^{+}$) calcd for $\mathrm{C}_{20} \mathrm{H}_{29} \mathrm{NNaO}^{+}[\mathrm{M}+\mathrm{Na}]^{+}$: 322.2141, found: 322.2141 .

IR (neat, cm ${ }^{-1}$ ): 2923, 2853, 1446, 1337, 1244, 996, 908, 800, 718.

\section{Non-1-en-4-ol (1t).}

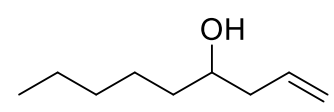

Compound 1t was prepared as a colorless oil in $84 \%$ yield $(0.60 \mathrm{~g}$, eluent: petroleum ether: $\mathrm{EtOAc}=30: 1)$ from hexanal $(0.50 \mathrm{~g}, 5.00 \mathrm{mmol})$ and allylmagnesium bromide $(7.5 \mathrm{~mL}, 1.0 \mathrm{M}$ in THF) following the above general procedure A. This compound was previously reported ${ }^{13}$.

$\mathbf{R}_{f}=0.49$ (petroleum ether: $\mathrm{EtOAc}=10: 1$ ) 
${ }^{1} \mathrm{H}$ NMR (400 MHz, $\left.\mathrm{CDCl}_{3}\right)$ ठ 5.89-5.73 (m, 1H), 5.21-4.97 (m, 2H), 3.71-3.45 (m, $1 \mathrm{H}), 2.38-2.00(\mathrm{~m}, 2 \mathrm{H}), 1.75(\mathrm{~s}, 1 \mathrm{H}), 1.55-1.37$ (m, 3H), 1.36-1.20 (m, 5H), 0.92$0.83(\mathrm{~m}, 3 \mathrm{H})$.

${ }^{13} \mathrm{C}$ NMR (100 MHz, $\left.\mathrm{CDCl}_{3}\right) \delta$ 135.1, 118.1, 70.8, 42.0, 36.9, 32.0, 25.5, 22.7, 14.1.

\section{1-Cyclohexylundec-10-en-1-ol (1u).}

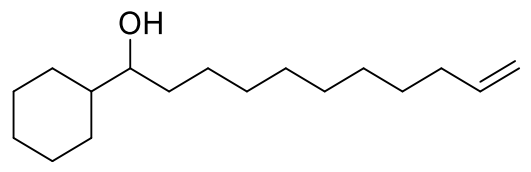

Compound $1 \mathbf{u}$ was prepared as a colorless oil in $72 \%$ yield $(0.97 \mathrm{~g}$, eluent: petroleum ether: $\mathrm{EtOAc}=30: 1)$ from undec-10-enal $(0.84 \mathrm{~g}, 5.00 \mathrm{mmol})$ and cyclohexylmagnesium chloride $(7.5 \mathrm{~mL}, 1.0 \mathrm{M}$ in $\mathrm{THF} /$ toluene $=62 / 38)$ following the above general procedure A.

$\mathbf{R}_{f}=0.35$ (petroleum ether: $\mathrm{EtOAc}=10: 1$ )

${ }^{1} \mathrm{H}$ NMR (400 MHz, $\left.\mathrm{CDCl}_{3}\right)$ ठ 5.90-5.73 (m, 1H), 5.02-4.88 (m, 2H), 3.48-3.22 (m, $1 \mathrm{H}), 2.12-1.97(\mathrm{~m}, 2 \mathrm{H}), 1.84-1.78(\mathrm{~m}, 1 \mathrm{H}), 1.79-1.72(\mathrm{~m}, 2 \mathrm{H}), 1.69-1.60(\mathrm{~m}, 2 \mathrm{H})$, $1.52-1.43(\mathrm{~m}, 2 \mathrm{H}), 1.40-1.18(\mathrm{~m}, 16 \mathrm{H}), 1.15-0.96(\mathrm{~m}, 3 \mathrm{H})$.

${ }^{13} \mathrm{C}$ NMR (100 MHz, $\left.\mathrm{CDCl}_{3}\right) \delta$ 139.3, 114.2, 76.3, 43.7, 34.3, 33.9, 29.9, 29.7, 29.6, $29.4,29.3,29.1,27.8,26.7,26.5,26.4,26.1$.

HRMS (ESI') calcd for $\mathrm{C}_{17} \mathrm{H}_{32} \mathrm{NaO}^{+}[\mathrm{M}+\mathrm{Na}]^{+}:$275.2345, found: 275.2345 .

IR (neat, cm-1): 2922, 2853, 1641, 1450, 992, 908, 637.

\section{1-Cyclopentylundec-10-en-1-ol (1v).}

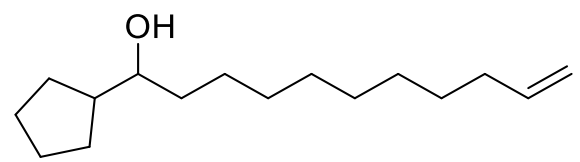

Compound $1 \mathbf{v}$ was prepared as a colorless oil in $61 \%$ yield $(0.73 \mathrm{~g}$, eluent: petroleum ether: $\mathrm{EtOAc}=30: 1)$ from undec-10-enal $(0.84 \mathrm{~g}, 5.00 \mathrm{mmol})$ and cyclopentylmagnesium bromide $(7.5 \mathrm{~mL}, 1.0 \mathrm{M}$ in THF) following the above general procedure A.

$\mathbf{R}_{f}=0.35$ (petroleum ether: $\mathrm{EtOAc}=10: 1$ ) 
${ }^{1} \mathrm{H}$ NMR (400 MHz, $\left.\mathrm{CDCl}_{3}\right)$ ठ 5.90-5.71 (m, 1H), 5.07-4.86 (m, 2H), 3.45-3.29 (m, 1H), 2.09-1.97 (m, 2H), 1.91-1.81 (m, 1H), 1.80-1.71 (m, 1H), 1.69-1.55 (m, 3H), $1.54-1.44(\mathrm{~m}, 4 \mathrm{H}), 1.41-1.23(\mathrm{~m}, 14 \mathrm{H}), 1.22-1.15$ (m, 1H).

${ }^{13} \mathrm{C}$ NMR (100 MHz, $\left.\mathrm{CDCl}_{3}\right) \delta$ 139.3, 114.2, 76.0, 46.5, 36.3, 33.9, 29.9, 29.7, 29.6, $29.29,29.25,29.0,28.6,25.9,25.8,25.7$.

HRMS (ESI ${ }^{+}$) calcd for $\mathrm{C}_{16} \mathrm{H}_{30} \mathrm{NaO}^{+}[\mathrm{M}+\mathrm{Na}]^{+}:$261.2189, found: 261.2188.

IR (neat, cm¹): 2924, 2855, 1641, 1456, 1311, 994, 908, 722, 635.

\section{1-Cyclopropylundec-10-en-1-ol (1w).}

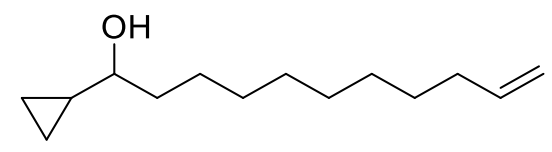

Compound 1w was prepared as a colorless oil in 54\% yield ( $0.57 \mathrm{~g}$, eluent: petroleum ether: $\mathrm{EtOAc}=30: 1)$ from undec-10-enal $(0.84 \mathrm{~g}, 5.00 \mathrm{mmol})$ and cyclopropylmagnesium bromide $(7.5 \mathrm{~mL}, 1.0 \mathrm{M}$ in THF) following the above general procedure $\mathrm{A}$.

$\mathbf{R}_{f}=0.35$ (petroleum ether: $\mathrm{EtOAc}=10: 1$ )

${ }^{1}$ H NMR (400 MHz, $\mathrm{CDCl}_{3}$ ) $\delta$ 5.87-5.68 (m, 1H), 5.02-4.84 (m, 2H), 2.90-2.77 (m, $1 \mathrm{H}), 2.08-1.96(\mathrm{~m}, 2 \mathrm{H}), 1.72(\mathrm{~s}, 1 \mathrm{H}), 1.61-1.50(\mathrm{~m}, 2 \mathrm{H}), 1.46-1.22(\mathrm{~m}, 12 \mathrm{H}), 0.94-$ $0.79(\mathrm{~m}, 1 \mathrm{H}), 0.56-0.40(\mathrm{~m}, 2 \mathrm{H}), 0.30-0.14(\mathrm{~m}, 2 \mathrm{H})$.

${ }^{13} \mathrm{C}$ NMR (100 MHz, $\left.\mathrm{CDCl}_{3}\right) \delta 139.3,114.2,76.9,37.4,33.9,29.8,29.7,29.5,29.2$, 29.0, 25.8, 18.1, 2.8, 2.5.

HRMS (ESI') calcd for $\mathrm{C}_{14} \mathrm{H}_{26} \mathrm{NaO}^{+}[\mathrm{M}+\mathrm{Na}]^{+}$: 233.1876, found: 233.1875 .

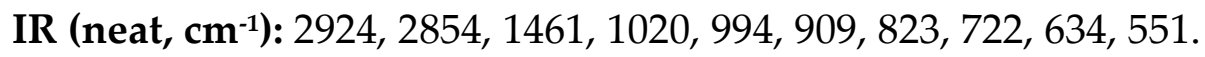

\section{2-Methyltridec-12-en-3-ol (1x).}

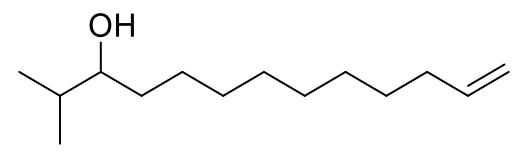

Compound $1 \mathrm{x}$ was prepared as a colorless oil in 77\% yield $(0.81 \mathrm{~g}$, eluent: petroleum ether: $\mathrm{EtOAc}=30: 1)$ from undec-10-enal $(0.84 \mathrm{~g}, 5.00 \mathrm{mmol})$ and isopropylmagnesium bromide $(2.7 \mathrm{~mL}, 2.8 \mathrm{M}$ in THF) following the above general procedure A. 
$\mathbf{R}_{f}=0.35$ (petroleum ether: $\mathrm{EtOAc}=10: 1$ )

${ }^{1} \mathrm{H}$ NMR (400 MHz, $\left.\mathrm{CDCl}_{3}\right)$ ठ 5.90-5.69 (m, 1H), 5.09-4.85 (m, 2H), 3.44-3.25 (m, 1H), 2.10-1.93 (m, 2H), 1.71-1.58 (m, 1H), 1.54-1.23 (m, 15H), 0.97-0.84 (m, 6H). ${ }^{13} \mathrm{C}$ NMR (100 MHz, $\left.\mathrm{CDCl}_{3}\right) \delta$ 139.3, 114.2, 76.8, 34.3, 33.9, 33.6, 29.9, 29.7, 29.6, 29.2, 29.0, 26.2, 19.0, 17.2.

HRMS (ESI') calcd for $\mathrm{C}_{14} \mathrm{H}_{28} \mathrm{NaO}^{+}[\mathrm{M}+\mathrm{Na}]^{+}$: 235.2032, found: 235.2032.

IR (neat, cm-1): 2925, 2855, 1463, 1369, 991, 908, 721, 634.

Tridec-12-en-3-ol (1y).

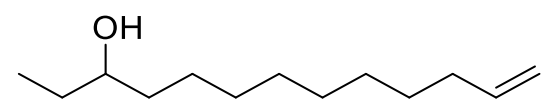

Compound 1y was prepared as a colorless oil in $82 \%$ yield $(0.81 \mathrm{~g}$, eluent: petroleum ether: $\mathrm{EtOAc}=30: 1)$ from undec-10-enal $(0.84 \mathrm{~g}, 5.00 \mathrm{mmol})$ and ethylmagnesium bromide (7.5 mL, 1.0 M in THF) following the above general procedure A. This compound was previously reported. ${ }^{14}$

$\mathbf{R}_{f}=0.35$ (petroleum ether: $\mathrm{EtOAc}=10: 1$ )

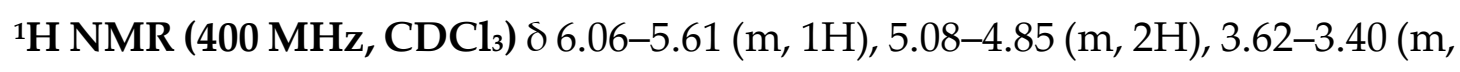
$1 \mathrm{H}), 2.13-1.95(\mathrm{~m}, 2 \mathrm{H}), 1.74-1.52(\mathrm{~m}, 1 \mathrm{H}), 1.56-1.23(\mathrm{~m}, 17 \mathrm{H}), 1.00-0.89(\mathrm{~m}, 3 \mathrm{H})$. ${ }^{13} \mathrm{C}$ NMR (100 MHz, $\left.\mathrm{CDCl}_{3}\right) \delta$ 139.3, 114.2, 73.4, 37.1, 33.9, 30.2, 29.8, 29.7, 29.6, $29.2,29.0,25.8,10.0$.

Dodec-11-en-2-ol (1z).

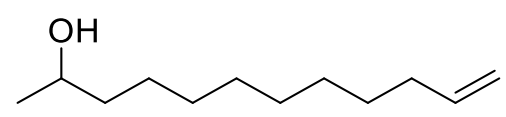

Compound $1 \mathrm{z}$ was prepared as a colorless oil in $50 \%$ yield $(0.46 \mathrm{~g}$, eluent: petroleum ether: $\mathrm{EtOAc}=30: 1)$ from undec-10-enal $(0.84 \mathrm{~g}, 5.00 \mathrm{mmol})$ and methylmagnesium bromide $\left(2.5 \mathrm{~mL}, 3.0 \mathrm{M}\right.$ in $\left.\mathrm{Et}_{2} \mathrm{O}\right)$ following the above general procedure A. This compound was previously reported..$^{15}$

$\mathbf{R}_{f}=0.38$ (petroleum ether: $\mathrm{EtOAc}=10: 1$ )

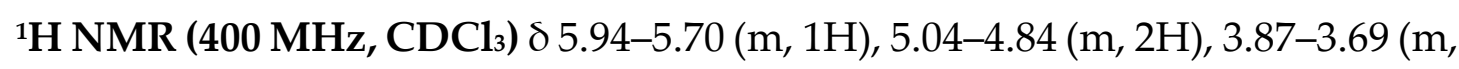
$1 \mathrm{H}), 2.09-1.99(\mathrm{~m}, 2 \mathrm{H}), 1.89-1.70(\mathrm{~m}, 1 \mathrm{H}), 1.48-1.24(\mathrm{~m}, 14 \mathrm{H}), 1.21-1.16(\mathrm{~m}, 3 \mathrm{H})$. 
${ }^{13} \mathrm{C}$ NMR (100 MHz, $\left.\mathrm{CDCl}_{3}\right) \delta$ 139.3, 114.2, 68.2, 39.4, 33.9, 29.73, 29.66, 29.5,

$29.2,29.0,25.9,23.5$.

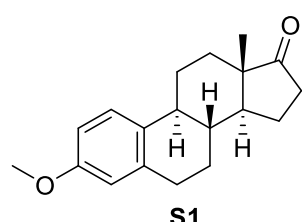

s1
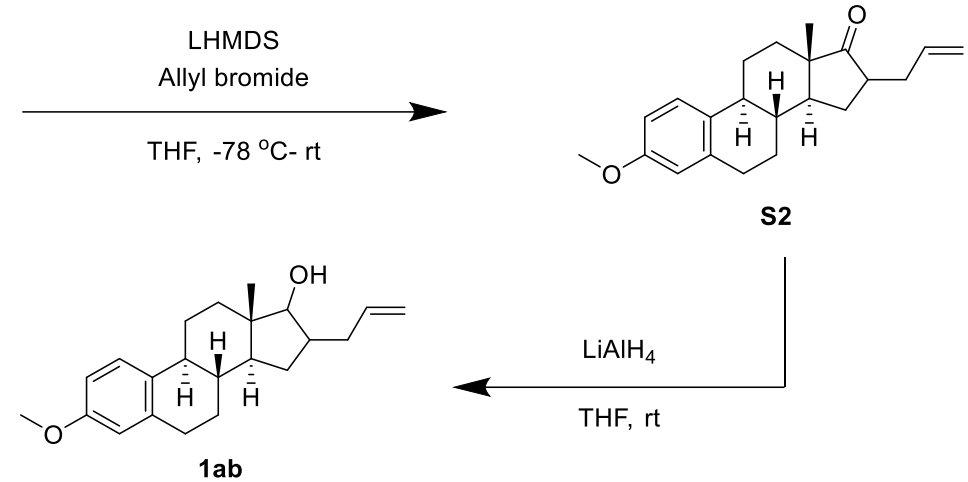

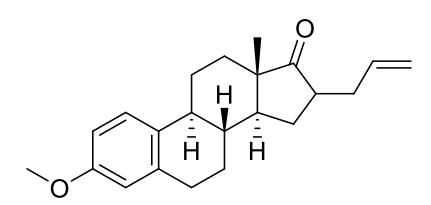

S2

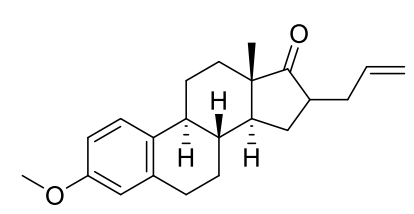

To a solution of $\mathbf{S 1} \mathbf{1}^{16}(0.85 \mathrm{~g}, 3.00 \mathrm{mmol})$ in THF $(20 \mathrm{~mL})$ was added LHMDS (3.3 $\mathrm{mL} 1.0 \mathrm{M}$ solution in $\mathrm{THF}$ ) at $-78{ }^{\circ} \mathrm{C}$ and stirred for $20 \mathrm{~min}$, then allyl bromide $(0.40 \mathrm{~g}, 3.30 \mathrm{mmol})$ was added dropwise. The resulting mixture was warmed up to rt and stirred for $6 \mathrm{~h}$, then quenched with saturated $\mathrm{NH}_{4} \mathrm{Cl}$, extracted with EtOAc, dried with $\mathrm{Na}_{2} \mathrm{SO}_{4}$, filtered, and concentrated, and the residue was purified by silica gel flash chromatography (petroleum ether: $\mathrm{EtOAc}=100: 1)$ to afford $0.33 \mathrm{~g}(34 \%)$ of $\mathbf{S} 2$ as a white solid.

$\mathbf{R}_{f}=0.36$ (petroleum ether: $\mathrm{EtOAc}=10: 1$ )

${ }^{1} \mathrm{H}$ NMR (400 MHz, $\left.\mathrm{CDCl}_{3}\right) \delta 7.21(\mathrm{~d}, J=8.6 \mathrm{~Hz}, 1 \mathrm{H}), 6.73(\mathrm{~d}, J=8.6 \mathrm{~Hz}, 1 \mathrm{H})$, $6.65(\mathrm{~s}, 1 \mathrm{H}), 5.88-5.72(\mathrm{~m}, 1 \mathrm{H}), 5.15-5.00(\mathrm{~m}, 2 \mathrm{H}), 3.78(\mathrm{~s}, 3 \mathrm{H}), 2.95-2.86(\mathrm{~m}, 2 \mathrm{H})$, 2.65-2.48 (m, 2H), 2.43-2.34 (m, 1H), 2.29-2.20 (m, 1H), 2.11-2.03 (m, 1H), 2.02$1.90(\mathrm{~m}, 2 \mathrm{H}), 1.87-1.78(\mathrm{~m}, 2 \mathrm{H}), 1.64-1.31(\mathrm{~m}, 5 \mathrm{H}), 0.96(\mathrm{~s}, 3 \mathrm{H})$.

${ }^{13} \mathrm{C}$ NMR (100 MHz, $\left.\mathrm{CDCl}_{3}\right) \delta$ 221.4, 157.7, 137.9, 136.6, 132.2, 126.4, 116.5, 114.0, 111.6, 55.3, 48.8, 48.1, 44.2, 44.1, 38.4, 35.2, 31.7, 29.8, 26.7, 26.6, 25.9, 14.7.

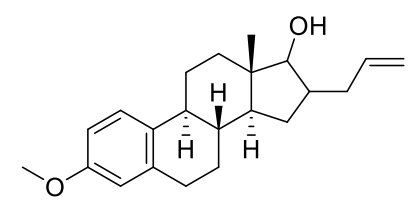


To a solution of $\mathrm{LiAlH}_{4}(0.15 \mathrm{~g}$, $3.90 \mathrm{mmol})$ in THF $(3.0 \mathrm{~mL})$ was added the solution of S2 $(0.62 \mathrm{~g}, 1.95 \mathrm{mmol})$ in THF $(1.0 \mathrm{~mL})$ at $-78{ }^{\circ} \mathrm{C}$ and stirred for 20 min, then quenched with water, extracted with EtOAc, dried with $\mathrm{Na}_{2} \mathrm{SO}_{4}$, filtered, and concentrated, and the residue was purified by silica gel flash chromatography (petroleum ether: EtOAc $=30: 1$ ) to afford $0.49 \mathrm{~g}(77 \%)$ of $1 \mathrm{ab}$ as a colorless oil.

$\mathbf{R}_{f}=0.21$ (petroleum ether: EtOAc $=10: 1$ )

${ }^{1} \mathrm{H}$ NMR $\left(400 \mathrm{MHz}, \mathrm{CDCl}_{3}\right) \delta 7.22(\mathrm{~d}, J=8.6 \mathrm{~Hz}, 1 \mathrm{H}), 6.73(\mathrm{~d}, J=8.5 \mathrm{~Hz}, 1 \mathrm{H})$, $6.65(\mathrm{~s}, 1 \mathrm{H}), 6.04-5.77(\mathrm{~m}, 1 \mathrm{H}), 5.28-4.73(\mathrm{~m}, 2 \mathrm{H}), 3.79(\mathrm{~s}, 3 \mathrm{H}), 3.42-3.28(\mathrm{~m}, 1 \mathrm{H})$, 2.96-2.79 (m, 2H), 2.45-2.27 (m, 2H), 2.25-2.11 (m, 2H), 1.99-1.83 (m, 3H), 1.60$1.39(\mathrm{~m}, 4 \mathrm{H}), 1.38-1.20(\mathrm{~m}, 4 \mathrm{H}), 0.83(\mathrm{~s}, 3 \mathrm{H})$.

${ }^{13} \mathrm{C}$ NMR (100 MHz, $\left.\mathrm{CDCl}_{3}\right) \delta$ d 157.5, 138.1, 137.9, 132.7, 126.4, 115.8, 113.9, 111.6, 87.5, 77.5, 77.2, 76.8, 55.3, 48.4, 44.2, 44.1, 43.4, 39.7, 38.7, 36.9, 29.9, 29.8, 27.4, 26.3, 12.0 .

HRMS (ESI') calcd for $\mathrm{C}_{22} \mathrm{H}_{31} \mathrm{O}_{2}{ }^{+}[\mathrm{M}+\mathrm{H}]^{+}: 327.2319$, found: 327.2318 .

IR (neat, cm $\left.{ }^{-1}\right)$ : 2924, 2867, 1610, 1500, 1455, 1311, 1280, 1255, 1239, 1144, 1036, 909.
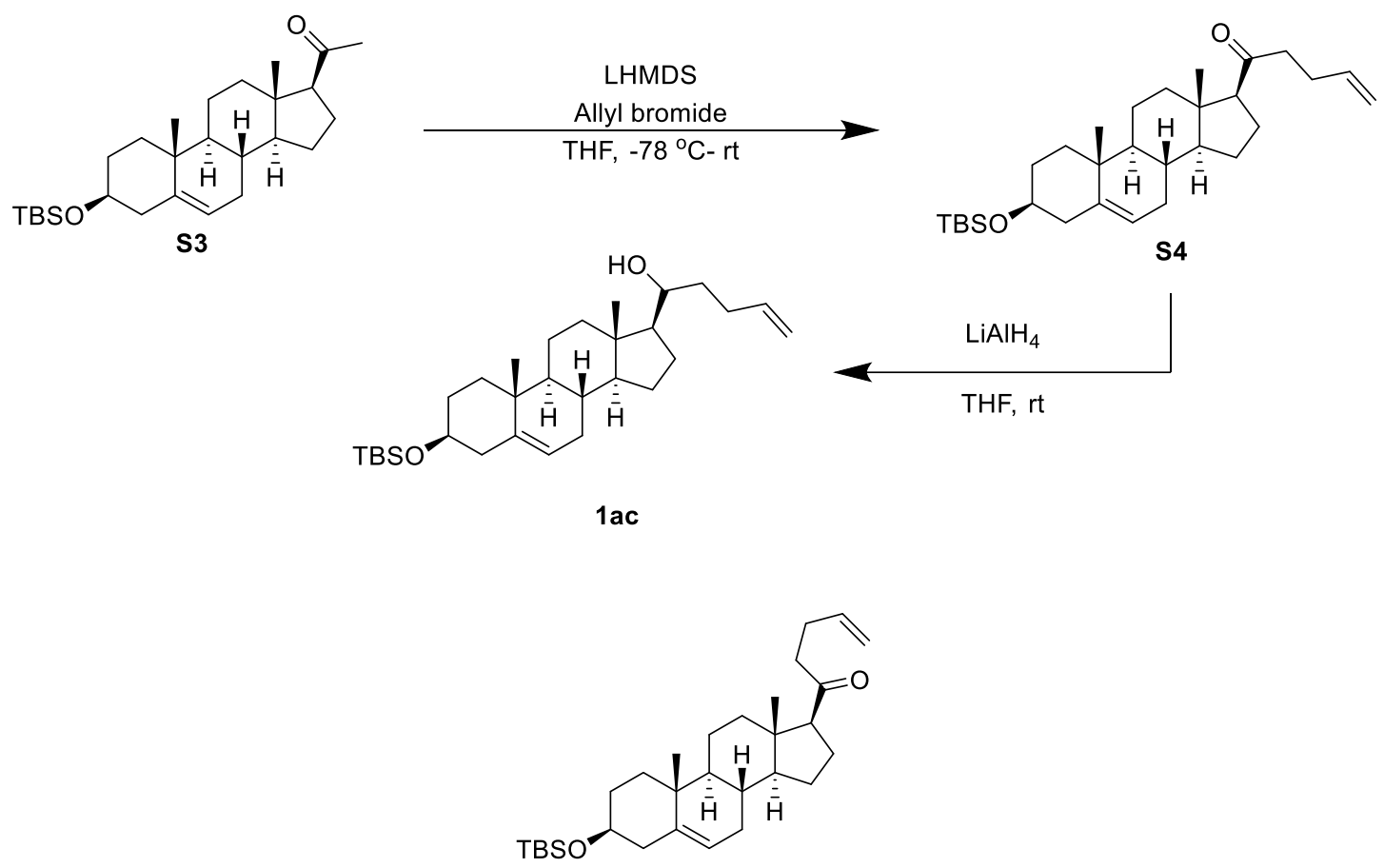

To a solution of $\mathbf{S 3}^{17}(1.29 \mathrm{~g}, 3.00 \mathrm{mmol})$ in THF $(20.0 \mathrm{~mL})$ was added LHMDS (3.3 mL, $1 \mathrm{M}$ in THF) at $-78^{\circ} \mathrm{C}$ and stirred for $20 \mathrm{~min}$, then allyl bromide $(0.40$ $\mathrm{g}, 3.30 \mathrm{mmol}$ ) was added dropwise. The resulting mixture was warmed up to 
rt and stirred for $6 \mathrm{~h}$, then quenched with saturated $\mathrm{NH}_{4} \mathrm{Cl}$, extracted with EtOAc, dried with $\mathrm{Na}_{2} \mathrm{SO}_{4}$, filtered, and concentrated, and the residue was purified by silica gel flash chromatography (petroleum ether: EtOAc $=100: 1$ ) to afford $0.94 \mathrm{~g}(66 \%)$ of $\mathrm{S} 4$ as colorless oil.

$\mathbf{R}_{f}=0.59$ (petroleum ether: EtOAc $=10: 1$ )

${ }^{1} \mathrm{H}$ NMR (400 MHz, $\left.\mathrm{CDCl}_{3}\right)$ ठ 5.98-5.67 (m, 1H), 5.37-5.28 (m, 1H), 5.13-4.88 (m, $2 \mathrm{H}), 3.56-3.36(\mathrm{~m}, 1 \mathrm{H}), 2.55-2.41(\mathrm{~m}, 3 \mathrm{H}), 2.37-2.10(\mathrm{~m}, 5 \mathrm{H}), 2.05-1.94(\mathrm{~m}, 2 \mathrm{H})$, $1.87-1.76(\mathrm{~m}, 1 \mathrm{H}), 1.74-1.39(\mathrm{~m}, 9 \mathrm{H}), 1.32-1.06(\mathrm{~m}, 4 \mathrm{H}), 0.99(\mathrm{~s}, 3 \mathrm{H}), 0.88(\mathrm{~s}, 9 \mathrm{H})$, $0.61(\mathrm{~s}, 3 \mathrm{H}), 0.05(\mathrm{~s}, 6 \mathrm{H})$.

${ }^{13} \mathrm{C}$ NMR (100 MHz, $\left.\mathrm{CDCl}_{3}\right) \delta$ 210.8, 141.7, 137.7, 121.0, 115.2, 72.7, 63.1, 57.2, $50.2,44.4,43.6,42.9,39.1,37.5,36.7,32.2,32.01,31.96,27.9,26.1,24.7,23.1,21.2$, $19.6,18.4,13.5,-4.4$.

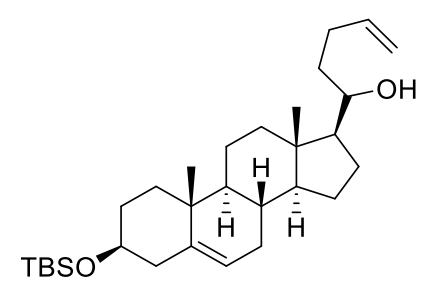

To a solution of $\mathrm{LiAlH}_{4}(24.0 \mathrm{mg}, 0.64 \mathrm{mmol})$ in THF $(3.0 \mathrm{~mL})$ was added the solution of $\mathbf{S} 4(0.15 \mathrm{~g}, 0.32 \mathrm{mmol})$ in THF $(1.0 \mathrm{~mL})$ at $-78{ }^{\circ} \mathrm{C}$ and stirred for 20 min, then quenched with water, extracted with EtOAc, dried with $\mathrm{Na}_{2} \mathrm{SO}_{4}$, filtered, and concentrated, and the residue was purified by silica gel flash chromatography (petroleum ether: $\mathrm{EtOAc}=30: 1$ ) to afford $0.11 \mathrm{~g}(70 \%)$ of $1 \mathrm{ac}$ as colorless oil.

$\mathbf{R}_{f}=0.31$ (petroleum ether: $\mathrm{EtOAc}=10: 1$ )

${ }^{1} \mathrm{H}$ NMR (400 MHz, $\left.\mathrm{CDCl}_{3}\right)$ ठ 5.97-5.73 (m, 1H), 5.39-5.22 (m, 1H), 5.12-4.83 (m, 2H), 3.64-3.53 (m, 1H), 3.51-3.40 (m, 1H), 2.35-2.04 (m, 5H), 2.01-1.90 (m, 1H), $1.82-1.75(\mathrm{~m}, 1 \mathrm{H}), 1.74-1.31(\mathrm{~m}, 13 \mathrm{H}), 1.29-1.06(\mathrm{~m}, 4 \mathrm{H}), 0.99(\mathrm{~s}, 4 \mathrm{H}), 0.88(\mathrm{~s}, 9 \mathrm{H})$, $0.75(\mathrm{~s}, 3 \mathrm{H}), 0.04(\mathrm{~s}, 6 \mathrm{H})$.

${ }^{13} \mathrm{C}$ NMR (100 MHz, $\left.\mathrm{CDCl}_{3}\right) \delta$ 141.7, 139.0, 121.1, 114.7, 74.0, 72.7, 56.7, 56.3, 50.3, $42.9,42.5,40.1,37.5,36.7,36.1,32.2,32.1,31.9,29.9,26.1,25.6,24.7,21.1,19.6$, $18.3,12.5,-4.5$.

HRMS (ESI') calcd for $\mathrm{C}_{30} \mathrm{H}_{52} \mathrm{NaO}_{2} \mathrm{Si}^{+}[\mathrm{M}+\mathrm{Na}]^{+}: 495.3629$, found: 495.3628 .

IR (neat, cm-1): 2931, 2859, 1465, 1379, 1252, 1094, 1005, 957, 911, 869, 837, 805, 776. 

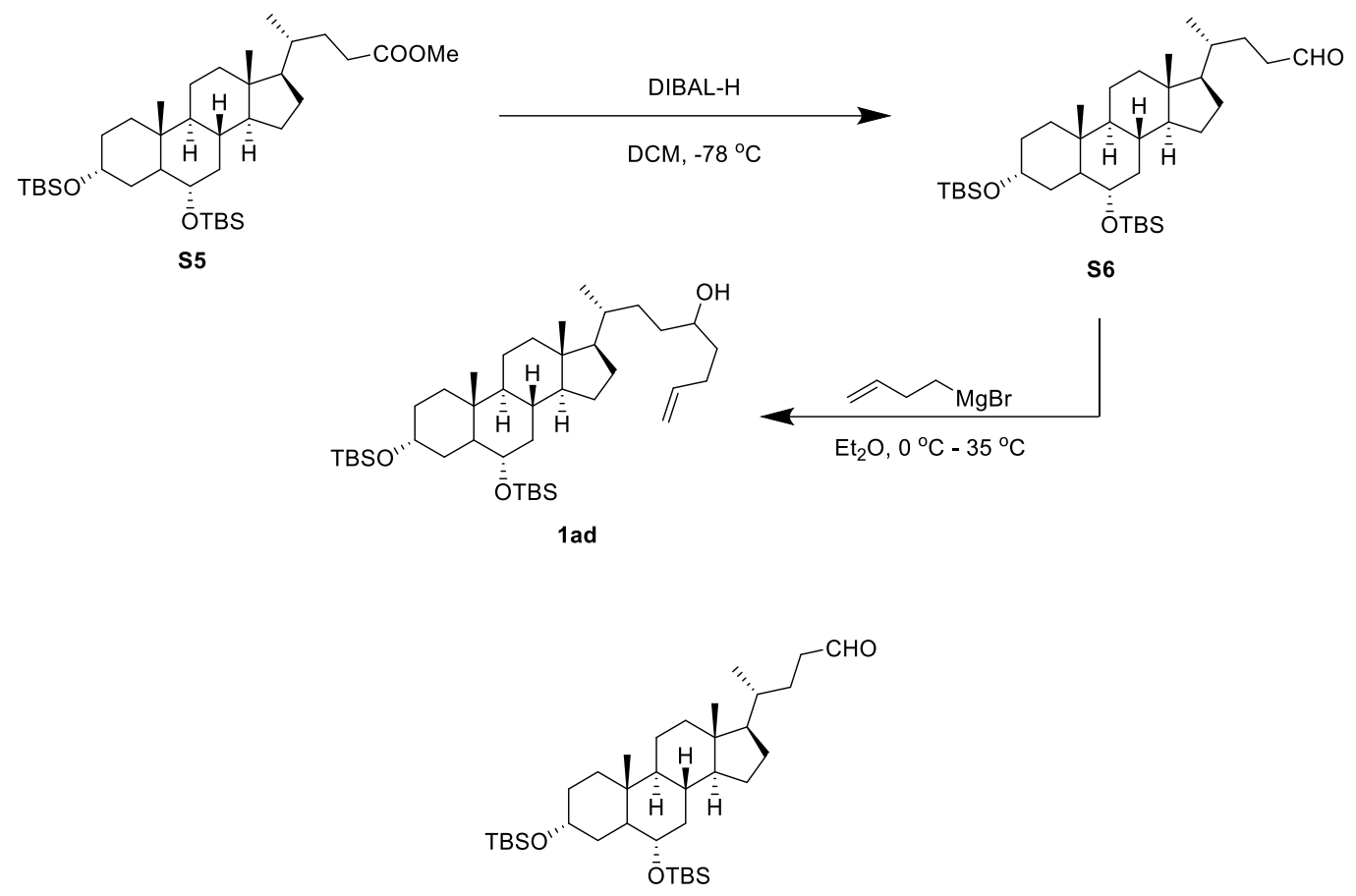

To a solution of S5 $(2.96 \mathrm{~g}, 4.67 \mathrm{mmol})$ in DCM $(30.0 \mathrm{~mL})$ was added DIBAL$\mathrm{H}$ ( $4.7 \mathrm{~mL} 1.5 \mathrm{M}$ in hexane) at $-78^{\circ} \mathrm{C}$ and stirred for $5 \mathrm{~h}$, then quenched with methanol, extracted with DCM, dried with $\mathrm{Na}_{2} \mathrm{SO}_{4}$, filtered, and concentrated, and the residue was purified by silica gel flash chromatography (petroleum ether: $\mathrm{EtOAc}=100: 1)$ to afford $1.88 \mathrm{~g}(65 \%)$ of $\mathbf{S 6}$ as a colorless oil.

$\mathbf{R}_{f}=0.67$ (petroleum ether: $\mathrm{EtOAc}=10: 1$ )

${ }^{1} \mathrm{H}$ NMR (400 MHz, CDCl $)$ ) $9.76(\mathrm{~s}, 1 \mathrm{H}), 4.07-3.86(\mathrm{~m}, 1 \mathrm{H}), 3.60-3.42(\mathrm{~m}, 1 \mathrm{H})$, $2.53-2.40(\mathrm{~m}, 1 \mathrm{H}), 2.40-2.27(\mathrm{~m}, 1 \mathrm{H}), 2.01-1.70(\mathrm{~m}, 5 \mathrm{H}), 1.47-1.23(\mathrm{~m}, 11 \mathrm{H}), 1.21-$ $0.98(\mathrm{~m}, 7 \mathrm{H}), 0.94-0.83(\mathrm{~m}, 25 \mathrm{H}), 0.63(\mathrm{~s}, 3 \mathrm{H}), 0.09-0.01(\mathrm{~m}, 12 \mathrm{H})$.

${ }^{13} \mathrm{C}$ NMR (100 MHz, $\mathrm{CDCl}_{3}$ ) $\delta$ 203.4, 73.1, 68.8, 56.3, 56.1, 49.7, 43.0, 41.1, 40.1, 39.7, 36.11, 36.06, 35.6, 35.5, 35.0, 31.2, 29.9, 28.3, 28.1, 26.2, 26.0, 24.3, 23.7, 20.9, $18.6,18.5,18.3,12.2,-4.3,-4.5,-4.6,-4.6$.

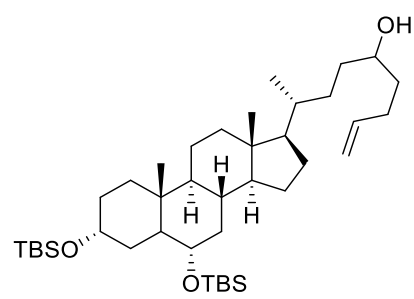


To a solution of $\mathrm{S} 6(0.56 \mathrm{~g}, 0.92 \mathrm{mmol})$ in $\mathrm{Et}_{2} \mathrm{O}(20.0 \mathrm{~mL})$ was added but-3-en1-ylmagnesium bromide $\left(2.8 \mathrm{~mL}, 0.5 \mathrm{M}\right.$ in THF) at $0{ }^{\circ} \mathrm{C}$. The resulting mixture was then warmed up to $35^{\circ} \mathrm{C}$ and stirred for $3 \mathrm{~h}$, then quenched with saturated $\mathrm{NH}_{4} \mathrm{Cl}$ at $0{ }^{\circ} \mathrm{C}$, extracted with EtOAc, dried with $\mathrm{Na}_{2} \mathrm{SO}_{4}$, filtered, and concentrated, and the residue was purified by silica gel flash chromatography (petroleum ether: EtOAc $=100: 1)$ to afford $2.21 \mathrm{~g}(67 \%)$ of $\mathbf{1 a d}$ as a colorless oil. $\mathbf{R}_{f}=0.43$ (petroleum ether: EtOAc $\left.=10: 1\right)$

${ }^{1} \mathrm{H}$ NMR (400 MHz, $\mathrm{CDCl}_{3}$ ) 8 5.96-5.75 (m, 1H), 5.14-4.94 (m, 2H), 4.03-3.89 (m, $1 \mathrm{H}), 3.64-3.43(\mathrm{~m}, 2 \mathrm{H}), 2.30-2.05(\mathrm{~m}, 2 \mathrm{H}), 2.00-1.91(\mathrm{~m}, 1 \mathrm{H}), 1.90-1.77(\mathrm{~m}, 2 \mathrm{H})$, $1.79-1.69(\mathrm{~m}, 1 \mathrm{H}), 1.58-1.51(\mathrm{~m}, 3 \mathrm{H}), 1.50-1.30(\mathrm{~m}, 12 \mathrm{H}), 1.28-0.99(\mathrm{~m}, 10 \mathrm{H})$, 0.93-0.84 (m, 24H), $0.63(\mathrm{~s}, 3 \mathrm{H}), 0.05(\mathrm{~s}, 6 \mathrm{H}), 0.02(\mathrm{~s}, 6 \mathrm{H})$.

${ }^{13} \mathrm{C}$ NMR (100 MHz, $\mathrm{CDCl}_{3}$ ) $\delta$ 138.8, 114.8, 73.1, 72.0, 68.8, 56.3, 56.2, 49.7, 43.0, $40.1,39.7,36.7,36.12,36.07,35.8,35.6,35.0,34.0,31.7,31.2,30.3,29.9,28.4,26.2$, 26.0, 24.4, 23.7, 20.9, 18.8, 18.6, 18.3, 12.2, -4.3, -4.5, -4.6, -4.6.

HRMS (ESI+) calcd for $\mathrm{C}_{40} \mathrm{H}_{76} \mathrm{NaO}_{3} \mathrm{Si}_{2}{ }^{+}[\mathrm{M}+\mathrm{Na}]^{+}: 683.5225$, found: 683.5223 .

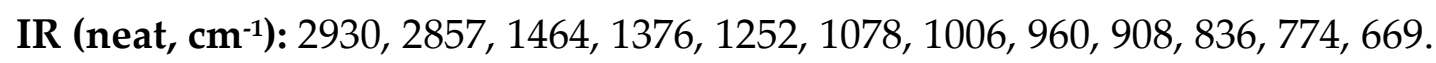

Synthesis of $(R)-1 a e$

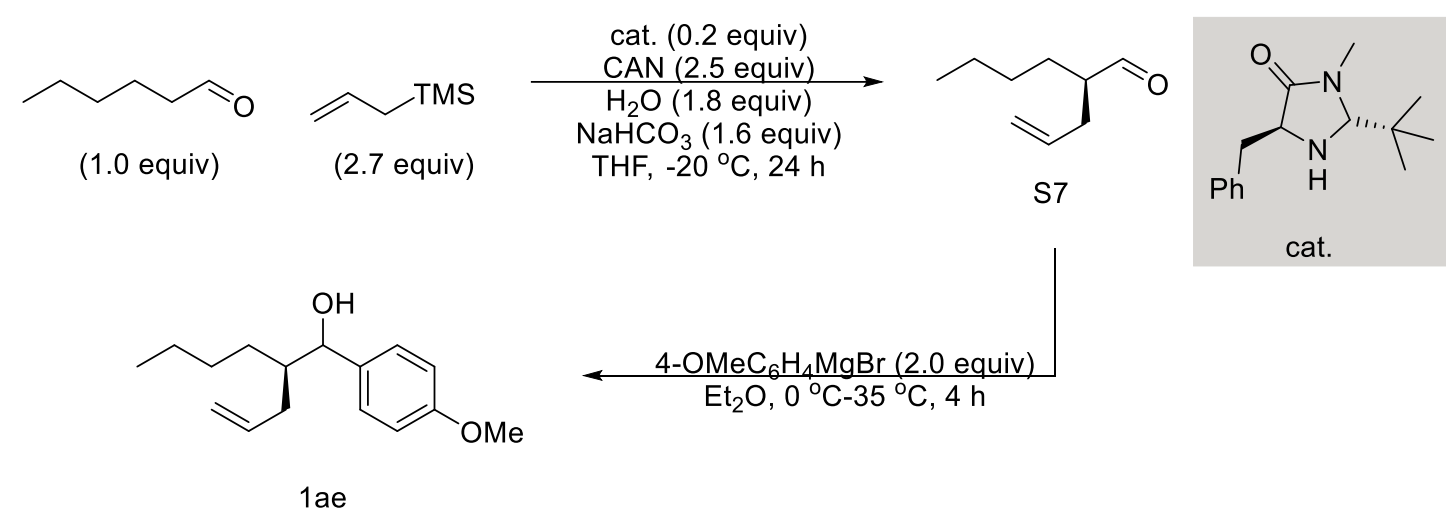

(R)-2-Allylhexanal (S7) ${ }^{18}$

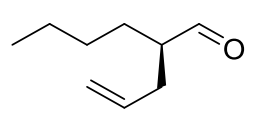

To a solution of (2S,5S)-5-benzyl-2-(tert-butyl)-3-methylimidazolidin-4-one ( $47 \mathrm{mg}, 0.19 \mathrm{mmol}), \mathrm{CAN}(1.28 \mathrm{~g}, 2.33 \mathrm{mmol}), \mathrm{NaHCO}_{3}(0.13 \mathrm{~g}, 1.49 \mathrm{mmol})$, and $\mathrm{H}_{2} \mathrm{O}(30.0 \mathrm{mg}, 1.67 \mathrm{mmol})$ in THF $(2.0 \mathrm{~mL})$ was added hexanal $(0.93 \mathrm{~g}, 0.93$ 
mmol) and allyltrimethylsilane $(0.29 \mathrm{~g}, 2.51 \mathrm{mmol})$ at $-20{ }^{\circ} \mathrm{C}$, The resulting mixture was stirred for $24 \mathrm{~h}$, then extracted with EtOAc, dried with $\mathrm{Na}_{2} \mathrm{SO}_{4}$, filtered, and concentrated to afford crude 57 , which was used in the next step without further purification.

\section{(2R)-2-Allyl-1-(4-methoxyphenyl)hexan-1-ol (1ae)}

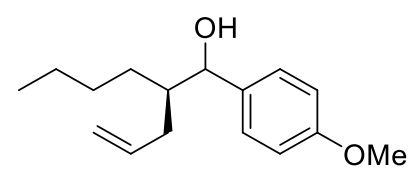

Compound $(\boldsymbol{R})$-1ae was prepared as a colorless oil in 22\% yield ( 2 steps, 51.0 $\mathrm{mg}, \mathrm{dr}=57: 43$, eluent: petroleum ether: EtOAc $=10: 1)$ from S7 and (4methoxyphenyl)magnesium bromide $\left(1.9 \mathrm{~mL}, 1.0 \mathrm{M}\right.$ in $\left.\mathrm{Et}_{2} \mathrm{O}\right)$ following the above general procedure A.

$\mathbf{R}_{f}=0.30$ (petroleum ether: $\mathrm{EtOAc}=5: 1$ )

${ }^{1} \mathrm{H}$ NMR (400 MHz, $\mathrm{CDCl}_{3}$ ) $\delta$ 7.25-7.19 (m, 2H), 6.94-6.82 (m, 2H), 5.95-5.66 (m, $1 \mathrm{H}), 5.16-4.92(\mathrm{~m}, 2 \mathrm{H}), 4.69-4.49(\mathrm{~m}, 1 \mathrm{H}), 3.90-3.73(\mathrm{~m}, 3 \mathrm{H}), 2.29-2.06(\mathrm{~m}, 2 \mathrm{H})$, 1.95-1.71 (m, 2H), 1.48-1.06 (m, 6H), 0.93-0.77 (m, 3H).

${ }^{13} \mathrm{C}$ NMR (100 MHz, $\left.\mathrm{CDCl}_{3}\right) \delta$ 158.9, 158.9, 137.4, 137.2, 135.9, 135.8, 127.8, 127.7, 116.33, 116.29, 113.70, 113.67, 76.0, 75.7, 55.4, 44.9, 44.8, 34.6, 33.5, 29.19, 29.15, 28.1, 23.1, 23.0, 14.21, 14.16 .

HRMS (ESI') calcd for $\mathrm{C}_{16} \mathrm{H}_{24} \mathrm{NaO}_{2}^{+}\left[\mathrm{M}+\mathrm{Na}^{+}\right]$: 271.1669, found: 271.1668.

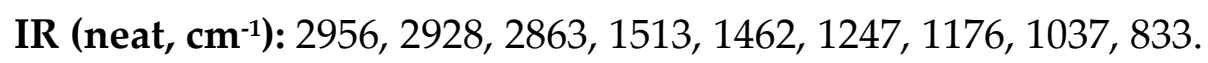

Enantiomeric excess [79\% ee (major), 79\% ee (minor)] was determined by chiral HPLC (Daicel Chiralcel AS-H, hexane/isopropyl alcohol $=99 / 1$, flow rate $=0.5$ $\mathrm{mL} / \mathrm{min}, \lambda=220 \mathrm{~nm}$ ) $t^{1}=16.9 \mathrm{~min}$ (major),$t^{2}=17.7 \mathrm{~min}$ (minor),$t^{3}=23.2 \mathrm{~min}$ (major), $t^{4}=24.4 \mathrm{~min}$ (minor).

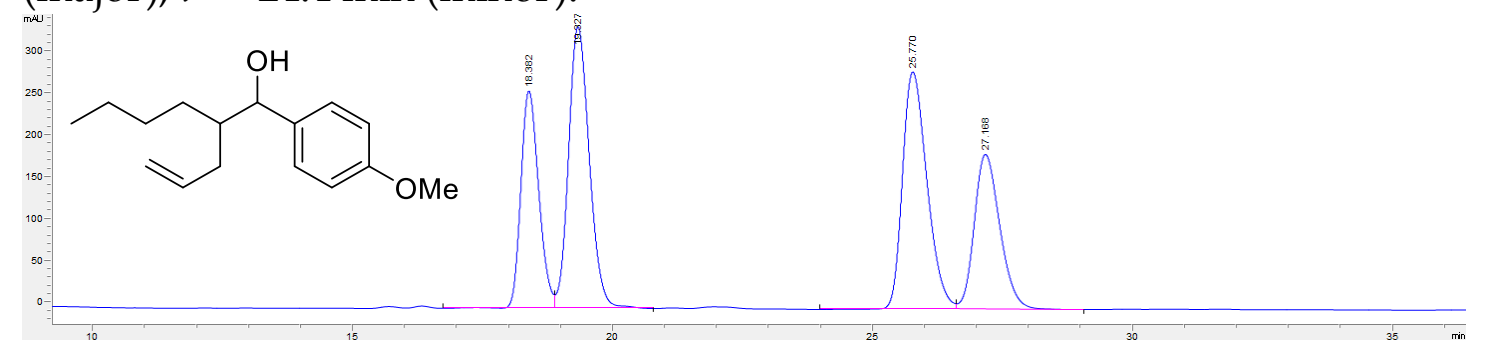


Signal 1: VWD1 A, Wavelength $=220 \mathrm{~nm}$
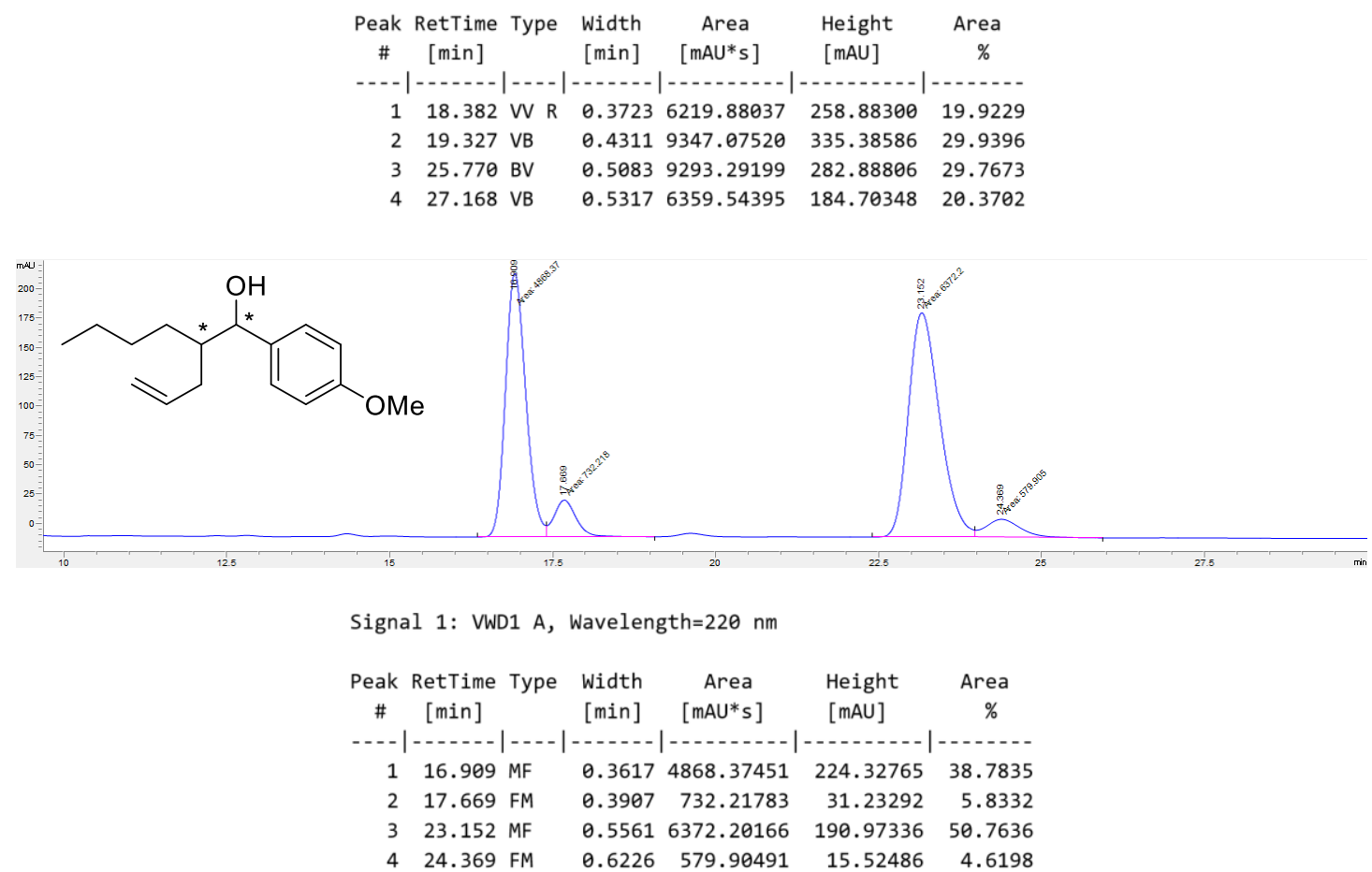

Synthesis of 1af-d<smiles>C=CCCC(=O)c1ccc(OC)cc1</smiles>

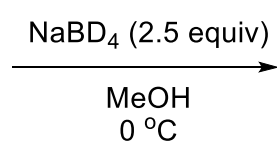<smiles>C=CCCC(O)(c1ccccc1)c1ccc(OC)cc1</smiles>

1-(4-Methoxyphenyl)pent-4-en-1-d-1-ol To a solution of 1-(4methoxyphenyl)pent-4-en-1-one ${ }^{19} 1$ af- $d(0.19 \mathrm{~g}, 1.00 \mathrm{mmol})$ in $\mathrm{MeOH}(2.0 \mathrm{~mL})$ was added $\mathrm{NaBD}_{4}(0.11 \mathrm{~g}, 2.50 \mathrm{mmol})$ at $-0{ }^{\circ} \mathrm{C}$, The resulting mixture was stirred for $0.5 \mathrm{~h}$, then extracted with EtOAc, dried with $\mathrm{Na}_{2} \mathrm{SO}_{4}$, then filtered, and concentrated, and the residue was purified by silica gel flash chromatography (petroleum ether: $\mathrm{EtOAc}=5: 1)$ to afford the title compound $(0.16 \mathrm{~g}, 81 \%$ yield). $\mathbf{R}_{f}=0.30$ (petroleum ether: $\mathrm{EtOAc}=5: 1$ )

${ }^{1}$ H NMR (400 MHz, $\mathrm{CDCl}_{3}$ ) $\delta$ 7.33-7.20 (m, 2H), 6.94-6.82 (m, 2H), 5.91-5.75 (m, $1 \mathrm{H}), 5.07-4.92(\mathrm{~m}, 2 \mathrm{H}), 4.63(\mathrm{t}, J=6.7 \mathrm{~Hz}, 0.03 \mathrm{H}), 3.80(\mathrm{~s}, 3 \mathrm{H}), 2.21-2.00(\mathrm{~m}, 2 \mathrm{H})$, 1.97-1.83 (m, 2H), 1.83-1.70 (m, 1H).

${ }^{13} \mathrm{C}$ NMR (100 MHz, $\left.\mathrm{CDCl}_{3}\right) \delta 159.2,138.4,136.8,127.3,115.0,114.0,73.3(\mathrm{t}, J=$ $21.8 \mathrm{~Hz}), 55.4,38.0,30.2$.

HRMS (EI+) calcd for $\mathrm{C}_{12} \mathrm{H}_{15} \mathrm{DO}_{2}^{+}\left[\mathrm{M}^{+}\right]$: 193.1208, found: 193.1211 .

IR (neat, cm-1): 2934, 1613, 1513, 1301, 1247, 1176, 1086, 1035, 913, 832. 
Synthesis of $\mathbf{1 a g}$

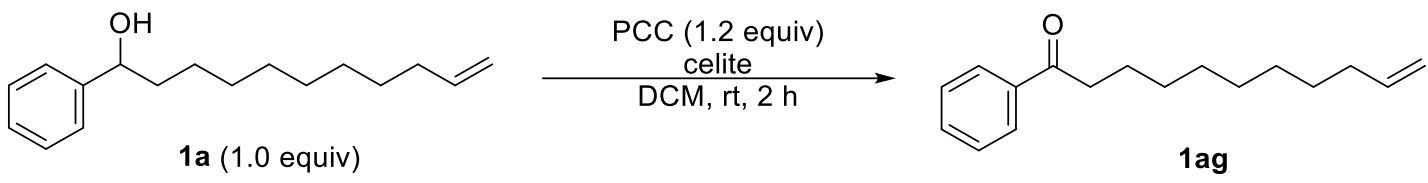

1-Phenylundec-10-en-1-one (1ag). To a solution of $1 \mathrm{a}(0.25 \mathrm{~g}, 1.00 \mathrm{mmol})$, celite $(0.50 \mathrm{~g})$ in DCM was added PCC $(0.26 \mathrm{~g}, 1.20 \mathrm{mmol})$ at $\mathrm{rt}$. The resulting mixture was stirred for $2 \mathrm{~h}$, then filtered, and concentrated, and the residue was purified by silica gel flash chromatography (petroleum ether : EtOAc $=10: 1$ ) to afford the title compound $(0.21 \mathrm{~g}, 88 \%$ yield $)$. This compound was previously reported. ${ }^{20}$

$\mathrm{R}_{f}=0.75$ (petroleum ether $: \mathrm{EtOAc}=20: 1$ )

${ }^{1} \mathrm{H}$ NMR (400 MHz, $\mathrm{CDCl}_{3}$ ) $\delta$ 7.97-7.90 (m, 2H), 7.56-7.47 (m, 1H), 7.47-7.38 (m, 2H), 5.87-5.71 (m, 1H), 5.03-4.87 (m, 2H), $2.93(\mathrm{t}, J=7.4 \mathrm{~Hz}, 2 \mathrm{H}), 2.07-1.97(\mathrm{~m}$, $2 \mathrm{H}), 1.78-1.68(\mathrm{~m}, 2 \mathrm{H}), 1.43-1.23(\mathrm{~m}, 10 \mathrm{H})$.

${ }^{13} \mathrm{C}$ NMR (100 MHz, $\left.\mathrm{CDCl}_{3}\right) \delta 200.4,139.1,137.1,132.8,128.5,128.0,114.2,38.6$, 33.8, 29.5, 29.4 (double), 29.1, 28.9, 24.3.

Synthesis of $\mathbf{1 a h}$
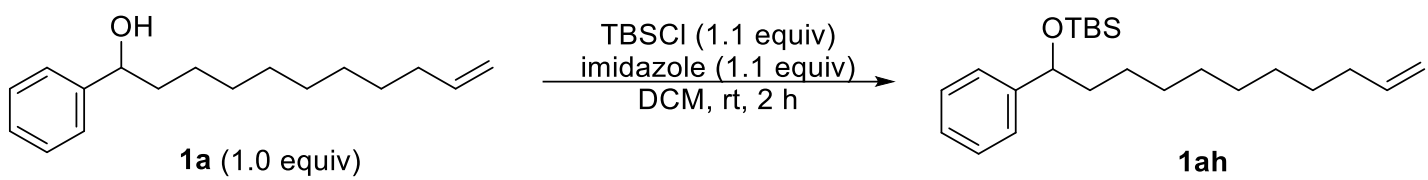

tert-Butyldimethyl((1-phenylundec-10-en-1-yl)oxy)silane (1ah). To a solution of $1 \mathrm{a}(0.49 \mathrm{~g}, 2.00 \mathrm{mmol}), \mathrm{TBSCl}(0.33 \mathrm{~g}, 2.20 \mathrm{mmol})$ in DCM was added imidazole $(0.15 \mathrm{~g}, 2.20 \mathrm{mmol})$ at $\mathrm{rt}$. The resulting mixture was stirred for $2 \mathrm{~h}$, then filtered, and concentrated, and the residue was purified by silica gel flash chromatography (petroleum ether) to afford the title compound $(0.38 \mathrm{~g}, 52 \%$ yield).

$\mathrm{R}_{f}=0.88$ (petroleum ether)

${ }^{1} \mathrm{H}$ NMR (400 MHz, $\left.\mathrm{CDCl}_{3}\right) \delta$ 7.45-7.15 (m, 5H), 5.95-5.73 (m, 1H), 5.13-4.84 (m, 
2H), 4.71-4.55 (m, 1H), 2.12-2.00 (m, 2H), 1.79-1.56 (m, 2H), 1.45-1.22 (m, 12H), $0.90(\mathrm{~s}, 9 \mathrm{H}), 0.04(\mathrm{~s}, 3 \mathrm{H}),-0.13(\mathrm{~s}, 3 \mathrm{H})$.

${ }^{13} \mathrm{C}$ NMR (100 MHz, $\left.\mathrm{CDCl}_{3}\right) \delta$ 146.1, 139.4, 128.1, 126.9, 126.0, 114.2, 75.3, 41.2, $34.0,29.70,29.68,29.6,29.3,29.1,26.0,25.8,18.4,-4.4,-4.8$.

HRMS (EI') calcd for $\mathrm{C}_{22} \mathrm{H}_{37} \mathrm{OSi}^{+}\left[{\mathrm{M}-\mathrm{CH}_{3}}^{+}\right]$: 345.2608, found: 345.2608 .

IR (neat, cm$^{-1}$ ): 2928, 2856, 1255, 1089, 1067, 836, 776, 700.

\section{Rh-Catalyzed Remote Isomerization of Alkenyl Alcohols}

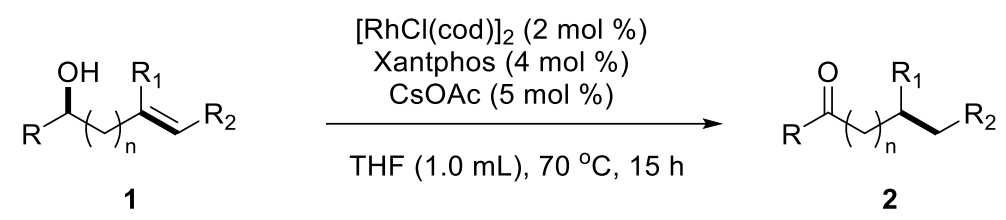

General Procedure C:

In glove box, to an oven-dried 4-mL vial were added $[\mathrm{RhCl}(\operatorname{cod})]_{2}(3.0 \mathrm{mg}$, $0.006 \mathrm{mmol})$, Xantphos $(6.9 \mathrm{mg}, 0.012 \mathrm{mmol})$, and anhydrous THF $(0.5 \mathrm{~mL})$. The resulting solution was stirred for $10 \mathrm{~min}$ at room temperature, at which time CsOAc (2.9 mg, $0.015 \mathrm{mmol})$ was added. The resulting mixture was stirred for an additional $10 \mathrm{~min}$, then the alkenyl alcohol 1 in THF $(0.5 \mathrm{~mL})$ was added. The vial was sealed with a teflon-lined cap, and the mixture was stirred at 70 ${ }^{\circ} \mathrm{C}$ in an oil bath for the duration of time indicated. The reaction mixture was filtered through celite, and washed with EtOAc. The filtrate was concentrated in vacuo, and the residue was purified by silica gel chromatography using petroleum ether/EtOAc as the eluent to afford the desired ketone 2.

1-Phenylundecan-1-one (2a).<smiles>CCCCCCCCCCC(=O)c1ccccc1</smiles> 
Compound 2a was prepared as a white solid in $97 \%$ yield $(71.6 \mathrm{mg}$, eluent: petroleum ether/EtOAc $=100 / 1$ to 50/1) from 1-phenylundec-10-en-1-ol 1a (73.8 $\mathrm{mg}, 0.3 \mathrm{mmol}),[\mathrm{RhCl}(\mathrm{cod})]_{2}(3.0 \mathrm{mg}, 0.006 \mathrm{mmol})$, Xantphos $(6.9 \mathrm{mg}, 0.012$ $\mathrm{mmol}$ ) and $\mathrm{CsOAc}(2.9 \mathrm{mg}, 0.015 \mathrm{mmol})$ following the above general procedure C. This compound was previously reported..$^{21}$ $\mathrm{R}_{f}=0.80$ (petroleum ether $/ \mathrm{EtOAc}=10 / 1$ )

${ }^{1} \mathrm{H}$ NMR $\left(400 \mathrm{MHz}, \mathrm{CDCl}_{3}\right) \delta 7.96(\mathrm{~d}, J=7.6 \mathrm{~Hz}, 2 \mathrm{H}), 7.54(\mathrm{t}, J=7.4 \mathrm{~Hz}, 1 \mathrm{H})$, $7.45(\mathrm{t}, J=7.6 \mathrm{~Hz}, 2 \mathrm{H}), 2.95(\mathrm{t}, J=7.5 \mathrm{~Hz}, 2 \mathrm{H}), 1.83-1.67(\mathrm{~m}, 2 \mathrm{H}), 1.48-1.19(\mathrm{~m}$, $14 \mathrm{H}), 0.88(\mathrm{t}, J=6.4 \mathrm{~Hz}, 3 \mathrm{H})$.

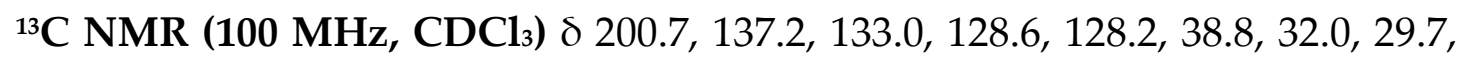
$29.64,29.62,29.5,29.4,24.5,22.8,14.2$.

\section{1-Phenylbutan-1-one (2b).}<smiles>CCCC(=O)c1ccccc1</smiles>

Compound $\mathbf{2 b}$ was prepared as a colorless oil in $88 \%$ yield (39.1 $\mathrm{mg}$, eluent: petroleum ether/EtOAc = 100/1 to 50/1) from 1-phenylbut-3-en-1-ol $\mathbf{1 b}(44.4 \mathrm{mg}$, $0.3 \mathrm{mmol}),[\mathrm{RhCl}(\mathrm{cod})]_{2}(3.0 \mathrm{mg}, 0.006 \mathrm{mmol})$, Xantphos $(6.9 \mathrm{mg}, 0.012 \mathrm{mmol})$ and $\mathrm{CsOAc}(2.9 \mathrm{mg}, 0.015 \mathrm{mmol})$ following the above general procedure $\mathrm{C}$. This compound was previously reported..$^{22}$

$\mathbf{R}_{f}=0.84$ (petroleum ether/EtOAc $=10 / 1$ )

${ }^{1} \mathrm{H}$ NMR $\left(400 \mathrm{MHz}, \mathrm{CDCl}_{3}\right) \delta 7.96(\mathrm{~d}, J=7.6 \mathrm{~Hz}, 2 \mathrm{H}), 7.54(\mathrm{t}, J=7.5 \mathrm{~Hz}, 1 \mathrm{H})$, $7.45(\mathrm{t}, J=7.7 \mathrm{~Hz}, 2 \mathrm{H}), 2.94(\mathrm{t}, J=7.4 \mathrm{~Hz}, 2 \mathrm{H}), 1.89-1.70(\mathrm{~m}, 2 \mathrm{H}), 1.00(\mathrm{t}, J=7.5$ $\mathrm{Hz}, 3 \mathrm{H})$.

${ }^{13} \mathrm{C}$ NMR (100 MHz, $\left.\mathrm{CDCl}_{3}\right) \delta$ \& 200.5, 137.1, 132.9, 128.6, 128.1, 40.6, 17.8, 14.0.

Compound $\mathbf{2 b}$ was prepared in 93\% yield $(41.3 \mathrm{mg}$ ) from (E)-1-phenylbut-2en-1-ol 1b' (44.4 mg, $0.3 \mathrm{mmol})$, [RhCl(cod)]2 (3.0 mg, $0.006 \mathrm{mmol})$, Xantphos (6.9 mg, $0.012 \mathrm{mmol})$, and CsOAc $(2.9 \mathrm{mg}, 0.015 \mathrm{mmol})$ following the above general procedure $C$.

\section{1-Phenylhexan-1-one (2c).}


<smiles>CCCCCC(=O)c1ccccc1</smiles>

Compound 2c was prepared as a colorless oil in $83 \%$ yield (43.8 $\mathrm{mg}$, eluent: petroleum ether $/ \mathrm{EtOAc}=100 / 1$ to 50/1) from 1-phenylhex-5-en-1-ol 1c (52.8 mg, $0.3 \mathrm{mmol}),[\mathrm{RhCl}(\mathrm{cod})]_{2}(3.0 \mathrm{mg}, 0.006 \mathrm{mmol})$, Xantphos (6.9 mg, $\left.0.012 \mathrm{mmol}\right)$, and $\mathrm{CsOAc}(2.9 \mathrm{mg}, 0.015 \mathrm{mmol})$ following the above general procedure $\mathrm{C}$. This compound was previously reported.22

$\mathrm{R}_{f}=0.84$ (petroleum ether $/$ EtOAc $=10 / 1$ )

${ }^{1} \mathrm{H}$ NMR (400 MHz, $\left.\mathrm{CDCl}_{3}\right) \delta 7.96(\mathrm{~d}, J=7.6 \mathrm{~Hz}, 2 \mathrm{H}), 7.54(\mathrm{t}, J=7.3 \mathrm{~Hz}, 1 \mathrm{H})$, $7.45(\mathrm{t}, J=7.6 \mathrm{~Hz}, 2 \mathrm{H}), 2.95(\mathrm{t}, J=7.4 \mathrm{~Hz}, 2 \mathrm{H}), 1.83-1.63(\mathrm{~m}, 2 \mathrm{H}), 1.44-1.23(\mathrm{~m}$, $4 \mathrm{H}), 1.01-0.85(\mathrm{~m}, 3 \mathrm{H})$.

${ }^{13} \mathrm{C}$ NMR (100 MHz, $\left.\mathrm{CDCl}_{3}\right) \delta$ 200.7, 137.2, 132.9, 128.6, 128.1, 38.7, 31.6, 24.2, $22.6,14.1$.

Compound $2 \mathrm{c}$ was prepared in 75\% yield $(39.6 \mathrm{mg})$ from $(E)-1$-phenylhex-4-en1-ol 1c' (52.8 mg, $0.3 \mathrm{mmol}),[\mathrm{RhCl}(\mathrm{cod})]_{2}$ (3.0 mg, $\left.0.006 \mathrm{mmol}\right)$, Xantphos (6.9 $\mathrm{mg}, 0.012 \mathrm{mmol})$, and CsOAc (2.9 mg, $0.015 \mathrm{mmol})$ following the above general procedure $\mathrm{C}$.

Compound $2 \mathrm{c}$ was prepared in 95\% yield (50.2 mg) from $(E)$-1-phenylhex-2-en1-ol 1c" (52.8 mg, $0.3 \mathrm{mmol}),[\mathrm{RhCl}(\mathrm{cod})]_{2}$ (3.0 mg, $\left.0.006 \mathrm{mmol}\right)$, Xantphos (6.9 $\mathrm{mg}, 0.012 \mathrm{mmol})$, and CsOAc $(2.9 \mathrm{mg}, 0.015 \mathrm{mmol})$ following the above general procedure C.

2-Methyl-1-phenylpropan-1-one (2d).<smiles>CC(C)C(=O)c1ccccc1</smiles>

Compound $2 \mathrm{~d}$ was prepared as a colorless oil in $89 \%$ yield $(39.5 \mathrm{mg}$, eluent: petroleum ether/EtOAc $=100 / 1$ to 50/1) from 2-methyl-1-phenylprop-2-en-1-ol 1d (44.4 mg, $0.3 \mathrm{mmol})$, [RhCl(cod) $]_{2}(3.0 \mathrm{mg}, 0.006 \mathrm{mmol})$, Xantphos (6.9 mg, 
$0.012 \mathrm{mmol})$, and $\mathrm{CsOAc}(2.9 \mathrm{mg}, 0.015 \mathrm{mmol})$ following the above general procedure $\mathrm{C}$. This compound was previously reported. ${ }^{23}$

$\mathrm{R}_{f}=0.87$ (petroleum ether $/$ EtOAc $=10 / 1$ )

${ }^{1} \mathrm{H}$ NMR (400 MHz, CDCl $\left.{ }_{3}\right) \delta 7.96(\mathrm{~d}, J=8.6 \mathrm{~Hz}, 2 \mathrm{H}), 7.55(\mathrm{t}, J=7.4 \mathrm{~Hz}, 1 \mathrm{H})$, $7.46(\mathrm{t}, J=7.4 \mathrm{~Hz}, 2 \mathrm{H}), 3.69-3.45(\mathrm{~m}, 1 \mathrm{H}), 1.26-1.17(\mathrm{~m}, 6 \mathrm{H})$.

${ }^{13} \mathrm{C}$ NMR (100 MHz, $\mathrm{CDCl}_{3}$ ) \& 204.6, 136.3, 132.9, 128.7, 128.4, 35.5, 19.3.

1-Phenyldecan-1-one (2e).

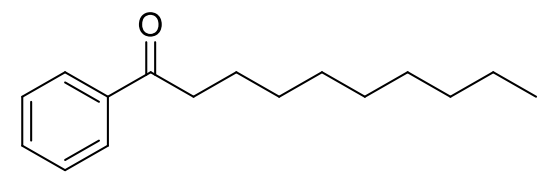

Compound 2e was prepared as a white solid in $92 \%$ yield $(64.0 \mathrm{mg}$, eluent: petroleum ether/EtOAc = 100/1 to 50/1) from (Z)-1-phenyldec-5-en-1-ol 1e (69.6 $\mathrm{mg}, 0.3 \mathrm{mmol}),[\mathrm{RhCl}(\mathrm{cod})]_{2}(3.0 \mathrm{mg}, 0.006 \mathrm{mmol})$, Xantphos $(6.9 \mathrm{mg}, 0.012$ $\mathrm{mmol})$, and CsOAc $(2.9 \mathrm{mg}, 0.015 \mathrm{mmol})$ following the above general procedure $\mathrm{C}$. This compound was previously reported..$^{24}$ $\mathrm{R}_{f}=0.80$ (petroleum ether $/$ EtOAc $=10 / 1$ )

${ }^{1} \mathrm{H}$ NMR (400 MHz, $\left.\mathrm{CDCl}_{3}\right) \delta 7.96(\mathrm{~d}, J=7.7 \mathrm{~Hz}, 2 \mathrm{H}), 7.54(\mathrm{t}, J=7.4 \mathrm{~Hz}, 1 \mathrm{H})$, $7.45(\mathrm{t}, J=7.6 \mathrm{~Hz}, 2 \mathrm{H}), 2.95(\mathrm{t}, J=7.5 \mathrm{~Hz}, 2 \mathrm{H}), 1.84-1.67(\mathrm{~m}, 2 \mathrm{H}), 1.51-1.17(\mathrm{~m}$, $12 \mathrm{H}), 0.94-0.81(\mathrm{~m}, 3 \mathrm{H})$.

${ }^{13} \mathrm{C}$ NMR (100 MHz, $\left.\mathrm{CDCl}_{3}\right) \delta$ 200.7, 137.2, 132.9, 128.6, 128.2, 38.8, 32.0, 29.6, 29.6, 29.5, 29.4, 24.5, 22.8, 14.2 .

Cyclohexyl(phenyl)methanone (2f).<smiles>O=C(c1ccccc1)C1CCCCC1</smiles>

Compound $2 \mathrm{f}$ was prepared as a white solid in $81 \%$ yield $(45.7 \mathrm{mg}$, eluent: petroleum ether/EtOAc $=100 / 1$ to 50/1) from cyclohex-3-en-1yl(phenyl)methanol 1f $(5: 4,56.4 \mathrm{mg}, 0.3 \mathrm{mmol})$, [RhCl(cod)]2 $(3.0 \mathrm{mg}, 0.006$ $\mathrm{mmol})$, Xantphos $(6.9 \mathrm{mg}, 0.012 \mathrm{mmol})$, and CsOAc (2.9 mg, $0.015 \mathrm{mmol})$ 
following the above general procedure C. This compound was previously reported. ${ }^{25}$

$\mathrm{R}_{f}=0.80$ (petroleum ether $/$ EtOAc $=10 / 1$ )

${ }^{1} \mathrm{H}$ NMR (400 MHz, $\left.\mathrm{CDCl}_{3}\right) \delta 7.94(\mathrm{~d}, J=7.6 \mathrm{~Hz}, 2 \mathrm{H}), 7.54(\mathrm{t}, 1 \mathrm{H}), 7.45(\mathrm{t}, J=7.1$ $\mathrm{Hz}, 2 \mathrm{H}), 3.34-3.18(\mathrm{~m}, 1 \mathrm{H}), 1.97-1.80(\mathrm{~m}, 4 \mathrm{H}), 1.79-1.64(\mathrm{~m}, 1 \mathrm{H}), 1.60-1.23$ (m, $5 \mathrm{H})$.

${ }^{13} \mathrm{C}$ NMR (100 MHz, $\left.\mathrm{CDCl}_{3}\right) \delta$ 204.0, 136.5, 132.8, 128.7, 128.4, 45.8, 29.6, 26.1, 26.0.

Bicyclo[2.2.1]heptan-2-yl(phenyl)methanone (2g).<smiles>O=C(c1ccccc1)C1CC2CCC1C2</smiles>

Compound $2 \mathrm{~g}$ was prepared as a colorless oil in $81 \%$ yield $(48.6 \mathrm{mg}$, eluent: petroleum ether/EtOAc $=100 / 1$ to $50 / 1, \mathrm{dr}=7.1: 1)$ from bicyclo[2.2.1] hept-5-en2-yl(phenyl)methanol $1 \mathrm{~g}$ (1:2.6:3.7:25.6, $60.0 \mathrm{mg}, 0.3 \mathrm{mmol}),[\mathrm{RhCl}(\mathrm{cod})]_{2}(7.4$ $\mathrm{mg}, 0.015 \mathrm{mmol})$, Xantphos (17.3 mg, $0.03 \mathrm{mmol})$, and CsOAc (5.8 mg, 0.03 $\mathrm{mmol}$ ) following the above general procedure $\mathrm{C}$. This compound was previously reported. ${ }^{26}$

$\mathrm{R}_{f}=0.88$ (petroleum ether $/$ EtOAc $=10 / 1$ )

${ }^{1} \mathrm{H}$ NMR (400 MHz, $\left.\mathrm{CDCl}_{3}\right) \delta 7.97(\mathrm{~d}, J=7.6 \mathrm{~Hz}, 2 \mathrm{H}), 7.54(\mathrm{t}, J=7.3 \mathrm{~Hz}, 1 \mathrm{H})$, $7.45(\mathrm{t}, J=7.5 \mathrm{~Hz}, 2 \mathrm{H}), 3.83-3.13(\mathrm{~m}, 1 \mathrm{H}), 2.67-2.45(\mathrm{~m}, 1 \mathrm{H}), 2.40-2.27(\mathrm{~m}, 1 \mathrm{H})$, 2.05-1.94 (m, 1H), 1.69-1.56 (m, 2H), 1.54-0.73 (m, 5H).

${ }^{13} \mathrm{C}$ NMR (100 MHz, $\left.\mathrm{CDCl}_{3}\right) \delta$ 201.7, 137.8, 132.7, 128.6, 128.4, 49.6, 42.1, 41.2, $37.6,30.9,29.1,24.5$.

1-(p-Tolyl)undecan-1-one (2h).

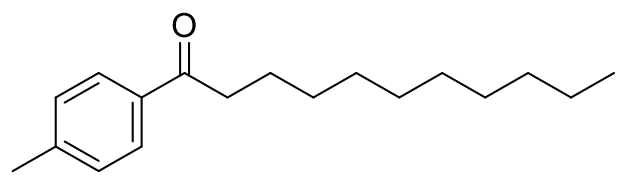

Compound $\mathbf{2 h}$ was prepared as a colorless oil in $92 \%$ yield $(71.8 \mathrm{mg}$, eluent: 
petroleum ether/EtOAc $=100 / 1$ to 50/1) from 1-(4-methylphenyl)undec-10-en1-ol $1 \mathrm{~g}$ (78.0 mg, $0.3 \mathrm{mmol})$, [RhCl(cod)]2 $(3.0 \mathrm{mg}, 0.006 \mathrm{mmol})$, Xantphos (6.9 $\mathrm{mg}, 0.012 \mathrm{mmol})$, and $\mathrm{CsOAc}(2.9 \mathrm{mg}, 0.015 \mathrm{mmol})$ following the above general procedure $\mathrm{C}$.

$\mathrm{R}_{f}=0.78$ (petroleum ether $/ \mathrm{EtOAc}=10 / 1$ )

${ }^{1} \mathrm{H}$ NMR (400 MHz, $\left.\mathrm{CDCl}_{3}\right) \delta 7.85(\mathrm{~d}, J=6.9 \mathrm{~Hz}, 2 \mathrm{H}), 7.24(\mathrm{~d}, J=7.9 \mathrm{~Hz}, 2 \mathrm{H})$, $2.92(\mathrm{t}, J=7.5 \mathrm{~Hz}, 2 \mathrm{H}), 2.40(\mathrm{~s}, 3 \mathrm{H}), 1.79-1.64(\mathrm{~m}, 2 \mathrm{H}), 1.42-1.21(\mathrm{~m}, 14 \mathrm{H}), 0.88$ $(\mathrm{t}, J=6.7 \mathrm{~Hz}, 3 \mathrm{H})$.

${ }^{13} \mathrm{C}$ NMR (100 MHz, $\left.\mathrm{CDCl}_{3}\right) \delta$ 200.4, 143.6, 134.7, 129.3, 128.3, 38.6, 32.0, 29.7, 29.64, 29.62, 29.5, 29.4, 24.6, 22.8, 21.7, 14.2.

HRMS (ESI') calcd for $\mathrm{C}_{18} \mathrm{H}_{29} \mathrm{O}^{+}[\mathrm{M}+\mathrm{H}]^{+}: 261.2213$, found: 261.2212 .

IR (neat, cm ${ }^{-1}$ ): 2924, 2854, 1683, 1608, 1461, 1409, 1363, 1292, 1206, 1180, 972, 806.

1-(3,5-Dimethylphenyl)undecan-1-one (2i).

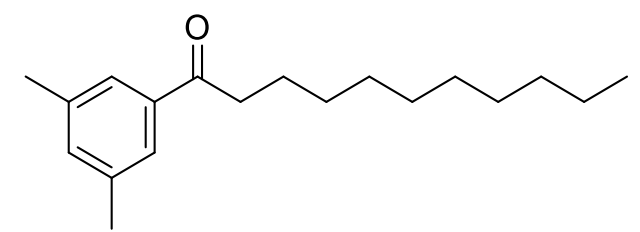

Compound $2 \mathbf{i}$ was prepared as a colorless oil in $80 \%$ yield $(65.8 \mathrm{mg}$, eluent: petroleum ether/EtOAc $=100 / 1$ to 50/1) from 1-(3,5-dimethylphenyl)undec-10en-1-ol (82.8 mg, $0.3 \mathrm{mmol})$, [RhCl(cod) $]_{2}(7.4 \mathrm{mg}, 0.015 \mathrm{mmol})$, Xantphos (17.3 $\mathrm{mg}, 0.03 \mathrm{mmol})$, and $\mathrm{CsOAc}(5.8 \mathrm{mg}, 0.03 \mathrm{mmol})$ following the above general procedure $\mathrm{C}$.

$\mathrm{R}_{f}=0.80$ (petroleum ether $/$ EtOAc $=10 / 1$ )

${ }^{1} \mathrm{H}$ NMR (400 MHz, $\left.\mathrm{CDCl}_{3}\right) \delta 7.55(\mathrm{~s}, 2 \mathrm{H}), 7.18(\mathrm{~s}, 1 \mathrm{H}), 2.92(\mathrm{t}, J=7.5 \mathrm{~Hz}, 2 \mathrm{H})$, $2.46-2.30(\mathrm{~m}, 6 \mathrm{H}), 1.77-1.65(\mathrm{~m}, 2 \mathrm{H}), 1.47-1.16(\mathrm{~m}, 14 \mathrm{H}), 0.88(\mathrm{t}, J=6.7 \mathrm{~Hz}, 3 \mathrm{H})$. ${ }^{13} \mathrm{C}$ NMR (100 MHz, $\left.\mathrm{CDCl}_{3}\right) \delta$ 201.2, 138.2, 137.4, 134.6, 126.0, 38.9, 32.0, 29.7, 29.7, 29.6, 29.52, 29.46, 24.6, 22.8, 21.4, 14.2.

HRMS (ESI+) calcd for $\mathrm{C}_{19} \mathrm{H}_{30} \mathrm{NaO}^{+}[\mathrm{M}+\mathrm{Na}]^{+}: 297.2189$, found: 297.2188 .

IR (neat, cm-1): 2922, 2854, 1685, 1605, 1460, 1376, 1300, 1181, 1159, 852, 686.

1-(4-Methoxyphenyl)undecan-1-one (2j). 


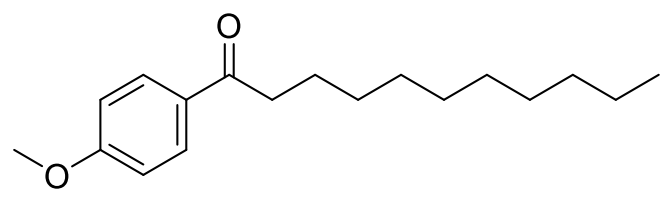

Compound $2 \mathbf{j}$ was prepared as a white solid in $97 \%$ yield $(80.3 \mathrm{mg}$, eluent: petroleum ether/EtOAc $=100 / 1$ to 50/1) from 1-(4-methoxy phenyl)undec-10-en1-ol 1j (82.8 mg, $0.3 \mathrm{mmol})$, [RhCl(cod)]2 (3.0 mg, $0.006 \mathrm{mmol})$, Xantphos (6.9 $\mathrm{mg}, 0.012 \mathrm{mmol})$, and $\mathrm{CsOAc}(2.9 \mathrm{mg}, 0.015 \mathrm{mmol})$ following the above general procedure $\mathrm{C}$. This compound was previously reported. ${ }^{21}$

$\mathrm{R}_{f}=0.55$ (petroleum ether $/$ EtOAc $=10 / 1$ )

${ }^{1} \mathrm{H}$ NMR $\left(400 \mathrm{MHz}, \mathrm{CDCl}_{3}\right) \delta 7.94(\mathrm{~d}, J=7.0 \mathrm{~Hz}, 2 \mathrm{H}), 6.93(\mathrm{~d}, J=7.0 \mathrm{~Hz}, 2 \mathrm{H})$, $3.86(\mathrm{~s}, 3 \mathrm{H}), 2.90(\mathrm{t}, J=7.1 \mathrm{~Hz}, 2 \mathrm{H}), 1.80-1.62(\mathrm{~m}, 2 \mathrm{H}), 1.49-1.15(\mathrm{~m}, 14 \mathrm{H}), 0.88$ $(\mathrm{t}, J=6.2 \mathrm{~Hz}, 3 \mathrm{H})$.

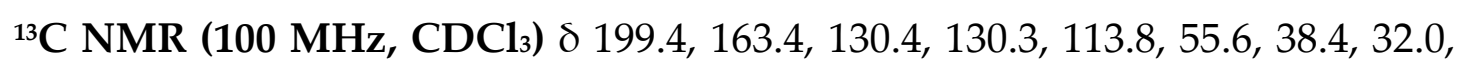
$29.72,29.65,29.63,29.58,29.5,24.8,22.8,14.2$.

1-(3,4-Dimethoxyphenyl)undecan-1-one (2k).

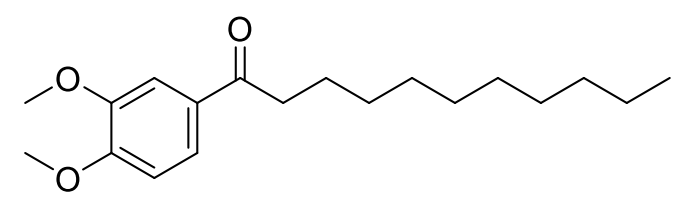

Compound 2k was prepared as a white solid in $98 \%$ yield $(90.0 \mathrm{mg}$, eluent: petroleum ether/EtOAc $=100 / 1$ to 50/1) from 1-(3,4-dimethoxyphenyl)undec10-en-1-ol 1k (91.8 mg, $0.3 \mathrm{mmol})$, [ RhCl(cod) ]2 (3.0 mg, $0.006 \mathrm{mmol})$, Xantphos (6.9 mg, $0.012 \mathrm{mmol})$, and CsOAc $(2.9 \mathrm{mg}, 0.015 \mathrm{mmol})$ following the above general procedure $\mathrm{C}$.

$\mathrm{R}_{f}=0.25$ (petroleum ether $/$ EtOAc $=10 / 1$ )

${ }^{1} \mathrm{H}$ NMR (400 MHz, $\left.\mathrm{CDCl}_{3}\right) \delta 7.58(\mathrm{~d}, J=8.4 \mathrm{~Hz}, 1 \mathrm{H}), 7.55-7.52(\mathrm{~m}, 1 \mathrm{H}), 6.88(\mathrm{~d}$, $J=8.3 \mathrm{~Hz}, 1 \mathrm{H}), 3.95-3.92(\mathrm{~m}, 6 \mathrm{H}), 2.91(\mathrm{t}, J=7.4 \mathrm{~Hz}, 2 \mathrm{H}), 1.78-1.65(\mathrm{~m}, 2 \mathrm{H}), 1.44-$ $1.20(\mathrm{~m}, 14 \mathrm{H}), 0.88(\mathrm{t}, J=6.8 \mathrm{~Hz}, 3 \mathrm{H})$.

${ }^{13} \mathrm{C}$ NMR (100 MHz, $\left.\mathrm{CDCl}_{3}\right) \delta$ S 199.3, 153.1, 149.1, 130.4, 122.7, 110.2, 110.0, 56.1, 56.0, 38.2, 32.0, 29.7, 29.60, 29.58, 29.5, 29.4, 24.9, 22.7, 14.2.

HRMS (ESI+) calcd for $\mathrm{C}_{19} \mathrm{H}_{30} \mathrm{NaO}_{3}{ }^{+}[\mathrm{M}+\mathrm{Na}]^{+}: 329.2087$, found: 329.2086 .

IR (neat, cm-1): 2924, 2853, 1675, 1590, 1514, 1461, 1415, 1343, 1264, 1202, 1152, 
$1024,879,806,764$.

1-(4-Fluorophenyl)undecan-1-one (21).

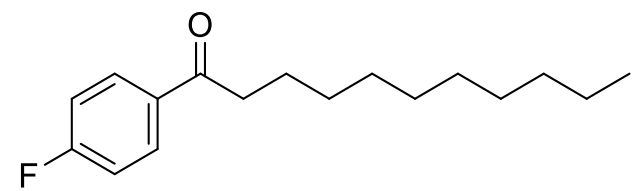

Compound 21 was prepared as a white solid in 94\% yield (74.4 mg, eluent: petroleum ether/EtOAc $=100 / 1$ to 50/1) from 1-(4-fluorophenyl)undec-10-en-1ol 11 (79.2 mg, $0.3 \mathrm{mmol})$, [RhCl(cod) ]2 (3.0 mg, $0.006 \mathrm{mmol})$, Xantphos (6.9 mg, $0.012 \mathrm{mmol})$, and CsOAc $(2.9 \mathrm{mg}, 0.015 \mathrm{mmol})$ following the above general procedure C. This compound was previously reported. ${ }^{27}$ $\mathrm{R}_{f}=0.80$ (petroleum ether $/$ EtOAc $=10 / 1$ )

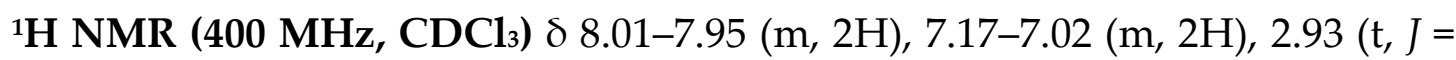
$7.4 \mathrm{~Hz}, 2 \mathrm{H}), 1.78-1.67(\mathrm{~m}, 2 \mathrm{H}), 1.43-1.21(\mathrm{~m}, 14 \mathrm{H}), 0.88(\mathrm{t}, J=6.7 \mathrm{~Hz}, 3 \mathrm{H})$.

${ }^{13} \mathrm{C}$ NMR (100 MHz, $\left.\mathrm{CDCl}_{3}\right) \delta$ 198.9, 165.7 (d, J = 254.2 Hz), $133.6(\mathrm{~d}, J=3.0 \mathrm{~Hz})$, $130.7(\mathrm{~d}, J=9.2 \mathrm{~Hz}), 115.7$ (d, J = 21.9 Hz), 38.6, 32.0, 29.7, 29.68, 29.61, 29.5, 29.4, $24.4,22.8,14.2$.

1-(4-Chlorophenyl)undecan-1-one (2m).<smiles>CCCCCCCCCCC(=O)c1ccc(Cl)cc1</smiles>

Compound $2 \mathrm{~m}$ was prepared as a white solid in $97 \%$ yield $(81.5 \mathrm{mg}$, eluent: petroleum ether/EtOAc $=100 / 1$ to 50/1) from 1-(4-chlorophenyl)undec-10-en-1ol $1 \mathrm{~m}$ (84.0 mg, $0.3 \mathrm{mmol})$, [ RhCl(cod) ]2 (3.0 mg, $0.006 \mathrm{mmol})$, Xantphos (6.9 mg, $0.012 \mathrm{mmol})$, and CsOAc (2.9 $\mathrm{mg}, 0.015 \mathrm{mmol})$ following the above general procedure $\mathrm{C}$. This compound was previously reported. ${ }^{27}$ $\mathrm{R}_{f}=0.80$ (petroleum ether $/$ EtOAc $=10 / 1$ )

${ }^{1} \mathrm{H}$ NMR (400 MHz, $\left.\mathrm{CDCl}_{3}\right) \delta 7.89(\mathrm{~d}, J=8.6 \mathrm{~Hz}, 2 \mathrm{H}), 7.41(\mathrm{~d}, J=8.3 \mathrm{~Hz}, 2 \mathrm{H})$, $2.92(\mathrm{t}, J=7.4 \mathrm{~Hz}, 2 \mathrm{H}), 1.77-1.66(\mathrm{~m}, 2 \mathrm{H}), 1.42-1.21(\mathrm{~m}, 14 \mathrm{H}), 0.88(\mathrm{t}, J=6.6 \mathrm{~Hz}$, $3 \mathrm{H})$. 
${ }^{13} \mathrm{C}$ NMR (100 MHz, $\left.\mathrm{CDCl}_{3}\right) \delta$ 199.3, 139.3, 135.5, 129.5, 128.9, 38.7, 32.0, 29.7, 29.60, 29.57, 29.4 (double), 24.4, 22.8, 14.2.

1-(4-(Trifluoromethyl)phenyl)undecan-1-one (2n).<smiles>CCCCCCCCCCC(=O)c1ccc(C(F)(F)F)cc1</smiles>

Compound 2n was prepared as a white solid in $98 \%$ yield (92.3 mg, eluent: petroleum ether/EtOAc $=100 / 1$ to 50/1) from 1-(4(trifluoromethyl)phenyl)undec-10-en-1-ol 1n (94.2 mg, $0.3 \mathrm{mmol})$, [RhCl(cod) $]_{2}$ (7.4 mg, $0.015 \mathrm{mmol})$, Xantphos (17.3 mg, $0.03 \mathrm{mmol}$ ), and CsOAc (5.8 mg, 0.03 $\mathrm{mmol}$ ) following the above general procedure $\mathrm{C}$. This compound was previously reported. ${ }^{21}$

$\mathrm{R}_{f}=0.83$ (petroleum ether $/$ EtOAc $=10 / 1$ )

${ }^{1} \mathrm{H}$ NMR (400 MHz, $\left.\mathrm{CDCl}_{3}\right) \delta 8.06(\mathrm{~d}, J=8.1 \mathrm{~Hz}, 2 \mathrm{H}), 7.72(\mathrm{~d}, J=8.1 \mathrm{~Hz}, 2 \mathrm{H})$, $2.99(\mathrm{t}, J=7.4 \mathrm{~Hz}, 2 \mathrm{H}), 1.83-1.69(\mathrm{~m}, 2 \mathrm{H}), 1.42-1.17(\mathrm{~m}, 14 \mathrm{H}), 0.88(\mathrm{t}, J=6.8 \mathrm{~Hz}$, $3 \mathrm{H})$.

${ }^{13} \mathrm{C}$ NMR (100 MHz, $\left.\mathrm{CDCl}_{3}\right) \delta$ 199.41, 139.71, 134.17 (q, $\left.J=32.6 \mathrm{~Hz}\right), 128.34$, $125.60(\mathrm{q}, J=3.7 \mathrm{~Hz}), 123.63(\mathrm{q}, J=271.5 \mathrm{~Hz}), 38.89,31.89,29.56,29.49,29.45$, $29.31,29.28,24.13,22.67,14.07$.

1-(6-((tert-Butyldimethylsilyl)oxy)naphthalen-2-yl)undecan-1-one (2o).<smiles>CCCCCCCCCCC(=O)c1ccc2cc(OC(C)C)ccc2c1</smiles>

Compound 20 was prepared as a white solid in 98\% yield (125.2 mg, eluent: petroleum ether/EtOAc $=100 / 1$ to 50/1) from 1-(6-((tertbutyldimethylsilyl)oxy)naphthalen-2-yl)undec-10-en-1-ol 10 (127.8 mg, 0.3 $\mathrm{mmol})$, [RhCl(cod)]2 (3.0 mg, $0.006 \mathrm{mmol})$, Xantphos $(6.9 \mathrm{mg}, 0.012 \mathrm{mmol})$, and CsOAc (2.9 mg, $0.015 \mathrm{mmol})$ following the above general procedure $\mathrm{C}$. $\mathrm{R}_{f}=0.75$ (petroleum ether $/$ EtOAc $=10 / 1$ ) 
${ }^{1} \mathrm{H}$ NMR (400 MHz, $\left.\mathrm{CDCl}_{3}\right) \delta 8.40(\mathrm{~s}, 1 \mathrm{H}), 7.99(\mathrm{~d}, J=8.7 \mathrm{~Hz}, 1 \mathrm{H}), 7.85(\mathrm{~d}, J=8.8$ $\mathrm{Hz}, 1 \mathrm{H}), 7.72(\mathrm{~d}, J=8.6 \mathrm{~Hz}, 1 \mathrm{H}), 7.21(\mathrm{~s}, 1 \mathrm{H}), 7.14(\mathrm{~d}, J=8.7 \mathrm{~Hz}, 1 \mathrm{H}), 3.06(\mathrm{t}, J=$ $7.5 \mathrm{~Hz}, 2 \mathrm{H}), 1.88-1.72(\mathrm{~m}, 2 \mathrm{H}), 1.46-1.24(\mathrm{~m}, 14 \mathrm{H}), 1.03(\mathrm{~s}, 9 \mathrm{H}), 0.89$ (t, J = 6.8 Hz, $3 \mathrm{H}), 0.28(\mathrm{~s}, 6 \mathrm{H})$.

${ }^{13} \mathrm{C}$ NMR (100 MHz, $\left.\mathrm{CDCl}_{3}\right) \delta$ 200.4, 155.9, 137.2, 132.8, 131.3, 129.6, 128.3, 127.1, 124.5, 123.0, 114.9, 38.7, 32.0, 29.7, 29.7, 29.6, 29.5, 25.8, 24.8, 22.8, 18.4, 14.3, -4.2.

HRMS (ESI') calcd for $\mathrm{C}_{27} \mathrm{H}_{42} \mathrm{NaO}_{2} \mathrm{Si}^{+}[\mathrm{M}+\mathrm{Na}]^{+}$: 449.2846, found: 449.2846.

IR (neat, cm-1): 2927, 2856, 1679, 1625, 1475, 1376, 1264, 1181, 974, 935, 872, 839, $812,782$.

1-(2-Methoxynaphthalen-1-yl)undecan-1-one (2p).

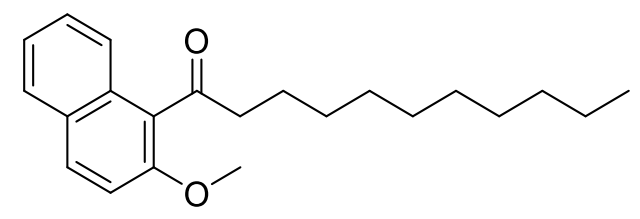

Compound $2 \mathbf{p}$ was prepared as a colorless oil in $61 \%$ yield $(59.7 \mathrm{mg}$, eluent: petroleum ether/EtOAc $=100 / 1$ to 50/1) from 1-(2-methoxynaphthalen-1yl)undec-10-en-1-ol 1p (97.8 mg, $0.3 \mathrm{mmol})$, [RhCl(cod)]2 (3.0 mg, $0.006 \mathrm{mmol}$ ), Xantphos (6.9 mg, $0.012 \mathrm{mmol})$, and CsOAc (2.9 mg, $0.015 \mathrm{mmol})$ following the above general procedure $\mathrm{C}$.

$\mathrm{R}_{f}=0.52$ (petroleum ether $/$ EtOAc $=10 / 1$ )

${ }^{1} \mathbf{H}$ NMR (400 MHz, $\left.\mathrm{CDCl}_{3}\right) \delta 7.85(\mathrm{~d}, J=9.1 \mathrm{~Hz}, 1 \mathrm{H}), 7.77(\mathrm{~d}, J=8.2 \mathrm{~Hz}, 1 \mathrm{H})$, $7.65(\mathrm{~d}, J=8.5 \mathrm{~Hz}, 1 \mathrm{H}), 7.44(\mathrm{t}, J=7.8 \mathrm{~Hz}, 1 \mathrm{H}), 7.34(\mathrm{t}, J=7.6 \mathrm{~Hz}, 1 \mathrm{H}), 7.25(\mathrm{~d}, J=$ $8.9 \mathrm{~Hz}, 1 \mathrm{H}), 3.92(\mathrm{~s}, 3 \mathrm{H}), 2.91(\mathrm{t}, J=7.5 \mathrm{~Hz}, 2 \mathrm{H}), 1.83-1.71(\mathrm{~m}, 2 \mathrm{H}), 1.51-1.10(\mathrm{~m}$, $14 \mathrm{H}), 0.88(\mathrm{t}, J=6.7 \mathrm{~Hz}, 3 \mathrm{H})$.

${ }^{13} \mathrm{C}$ NMR (100 MHz, $\left.\mathrm{CDCl}_{3}\right) \delta$ 208.2, 153.6, 131.2, 130.7, 128.9, 128.2, 127.6, 125.5, 124.1, 123.7, 112.9, 56.5, 45.3, 32.0, 29.7, 29.6, 29.6, 29.4, 24.0, 22.8, 14.2.

HRMS (ESI') calcd for $\mathrm{C}_{22} \mathrm{H}_{30} \mathrm{NaO}_{2}{ }^{+}[\mathrm{M}+\mathrm{Na}]^{+}:$349.2138, found: 349.2137.

IR (neat, cm-1): 2924, 2853, 1695, 1594, 1511, 1464, 1435, 1342, 1273, 1252, 1183, 1152, 1071, 809, 747.

1-(Furan-2-yl)pentan-1-one (2q) 


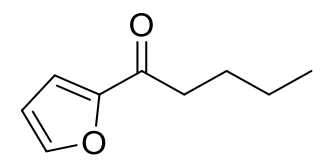

Compound 2q was prepared as a colorless oil in $95 \%$ yield $(43.0 \mathrm{mg}$, eluent: petroleum ether/ $\mathrm{Et}_{2} \mathrm{O}=50 / 1$ to 30/1) from 1-(furan-2-yl)pent-4-en-1-ol $\mathbf{1 q}$ (45.6 $\mathrm{mg}, 0.3 \mathrm{mmol}),[\mathrm{RhCl}(\mathrm{cod})]_{2}(3.0 \mathrm{mg}, 0.006 \mathrm{mmol})$, Xantphos $(6.9 \mathrm{mg}, 0.012$ $\mathrm{mmol})$, and CsOAc $(2.9 \mathrm{mg}, 0.015 \mathrm{mmol})$ following the above general procedure $\mathrm{C}$. This compound was previously reported. ${ }^{28}$ $\mathrm{R}_{f}=0.82$ (petroleum ether $/ \mathrm{Et}_{2} \mathrm{O}=20 / 1$ )

${ }^{1} \mathrm{H}$ NMR (400 MHz, $\left.\mathrm{CDCl}_{3}\right)$ $\delta$ 7.63-7.51 (m, 1H), 7.23-7.14 (m, 1H), 6.56-6.50 (m, $1 \mathrm{H}), 2.82(\mathrm{t}, J=7.6 \mathrm{~Hz}, 2 \mathrm{H}), 1.77-1.65(\mathrm{~m}, 2 \mathrm{H}), 1.48-1.33(\mathrm{~m}, 2 \mathrm{H}), 0.94(\mathrm{t}, J=7.5$ $\mathrm{Hz}, 3 \mathrm{H})$.

${ }^{13} \mathrm{C}$ NMR (100 MHz, $\left.\mathrm{CDCl}_{3}\right) \delta$ 190.0, 153.0, 146.3, 116.9, 112.2, 38.4, 26.6, 22.6, 14.0.

1-(Thiophen-2-yl)pentan-1-one (2r)

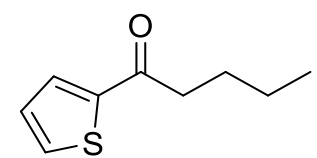

Compound $2 \mathbf{r}$ was prepared as a colorless oil in $80 \%$ yield $(35 \mathrm{mg}$, eluent: petroleum ether $/ \mathrm{Et}_{2} \mathrm{O}=50 / 1$ to 30/1) from 1-(thiophen-2-yl)pent-4-en-1-ol $1 \mathrm{r}$ (43 $\mathrm{mg}, 0.26 \mathrm{mmol})$, [ $\mathrm{RhCl}(\mathrm{cod})]_{2}(3.0 \mathrm{mg}, 0.006 \mathrm{mmol})$, Xantphos $(6.9 \mathrm{mg}, 0.012$ $\mathrm{mmol})$, and CsOAc $(2.9 \mathrm{mg}, 0.015 \mathrm{mmol})$ following the above general procedure $\mathrm{C}$. This compound was previously reported. ${ }^{29}$

$\mathrm{R}_{f}=0.91$ (petroleum ether $/ \mathrm{Et}_{2} \mathrm{O}=20 / 1$ )

${ }^{1} \mathrm{H}$ NMR (400 MHz, $\left.\mathrm{CDCl}_{3}\right) \delta$ 7.75-7.68 (m, 1H), 7.67-7.59 (m, 1H), 7.19-7.05 (m, $1 \mathrm{H}), 2.90(\mathrm{t}, J=7.4 \mathrm{~Hz}, 2 \mathrm{H}), 1.81-1.67(\mathrm{~m}, 2 \mathrm{H}), 1.47-1.24(\mathrm{~m}, 2 \mathrm{H}), 0.95(\mathrm{t}, J=7.3$ $\mathrm{Hz}, 3 \mathrm{H})$.

${ }^{13} \mathrm{C}$ NMR (100 MHz, $\left.\mathrm{CDCl}_{3}\right) \delta$ 193.7, 144.6, 133.4, 131.8, 128.1, 39.3, 27.0, 22.6, 14.0.

1-(1-Methyl-1H-indol-5-yl)undecan-1-one (2s). 


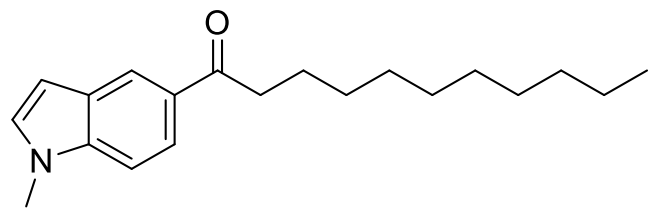

Compound 2s was prepared as a yellow solid in $84 \%$ yield $(75.3 \mathrm{mg}$, eluent: petroleum ether $/$ EtOAc $=100 / 1$ to 50/1) from 1-(1-methyl-1H-indol-5-yl)undec10-en-1-ol 1s ( $89.7 \mathrm{mg}, 0.3 \mathrm{mmol})$, [RhCl(cod)]2 (3.0 mg, $0.006 \mathrm{mmol})$, Xantphos (6.9 $\mathrm{mg}, 0.012 \mathrm{mmol})$, and $\mathrm{CsOAc}(2.9 \mathrm{mg}, 0.015 \mathrm{mmol})$ following the above general procedure $\mathrm{C}$.

$\mathrm{R}_{f}=0.80$ (petroleum ether $/ \mathrm{EtOAc}=10 / 1$ )

${ }^{1} \mathrm{H}$ NMR (400 MHz, CDCl $)$ ) $8.29(\mathrm{~s}, 1 \mathrm{H}), 7.89(\mathrm{~d}, J=8.7 \mathrm{~Hz}, 1 \mathrm{H}), 7.29(\mathrm{~d}, J=8.6$ $\mathrm{Hz}, 1 \mathrm{H}), 7.07(\mathrm{~d}, J=3.2 \mathrm{~Hz}, 1 \mathrm{H}), 6.58(\mathrm{~d}, J=3.1 \mathrm{~Hz}, 1 \mathrm{H}), 3.76(\mathrm{~s}, 3 \mathrm{H}), 3.01(\mathrm{t}, J=$ $7.5 \mathrm{~Hz}, 2 \mathrm{H}), 1.83-1.69(\mathrm{~m}, 2 \mathrm{H}), 1.45-1.19(\mathrm{~m}, 14 \mathrm{H}), 0.88(\mathrm{t}, J=6.7 \mathrm{~Hz}, 3 \mathrm{H})$.

${ }^{13} \mathrm{C}$ NMR (100 MHz, $\left.\mathrm{CDCl}_{3}\right) \delta$ 200.8, 139.1, 130.4, 129.3, 128.0, 122.8, 121.8, 109.1, 103.0, 38.6, 33.0, 32.0, 29.7, 29.63 (double), 29.61, 29.4, 25.1, 22.8, 14.2.

HRMS (ESI') calcd for $\mathrm{C}_{20} \mathrm{H}_{30} \mathrm{NO}^{+}[\mathrm{M}+\mathrm{H}]^{+}: 300.2322$, found: 300.2322 .

IR (neat, cm-1): 2923, 2853, 1669, 1608, 1450, 1367, 1341, 1290, 1247, 1154, 1096, 721.

Nonan-4-one (2t).

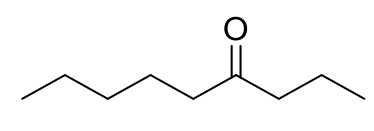

Compound $2 \mathbf{t}$ was prepared as a colorless oil in $80 \%$ yield $(34.1 \mathrm{mg}$, eluent: petroleum ether/EtOAc $=100 / 1$ to 50/1) from non-1-en-4-ol 1t $(42.6 \mathrm{mg}, 0.3$ $\mathrm{mmol}),[\mathrm{RhCl}(\mathrm{cod})]_{2}(3.0 \mathrm{mg}, 0.006 \mathrm{mmol})$, Xantphos (6.9 mg, $\left.0.012 \mathrm{mmol}\right)$, and CsOAc ( $2.9 \mathrm{mg}, 0.015 \mathrm{mmol}$ ) following the above general procedure C. This compound was previously reported. ${ }^{30}$

$\mathrm{R}_{f}=0.91$ (petroleum ether/EtOAc $=10 / 1$ )

${ }^{1} \mathrm{H}$ NMR (400 MHz, $\left.\mathrm{CDCl}_{3}\right) \delta$ 2.47-2.31 (m, 4H), 1.68-1.50 (m, 4H), 1.36-1.20 (m, $4 \mathrm{H}), 0.98-0.81(\mathrm{~m}, 6 \mathrm{H})$.

${ }^{13} \mathrm{C}$ NMR (100 MHz, $\left.\mathrm{CDCl}_{3}\right)$ o 211.7, 44.8, 42.9, 31.6, 23.7, 22.6, 17.4, 14.0, 13.9.

\section{1-Cyclohexylundecan-1-one (2u).}




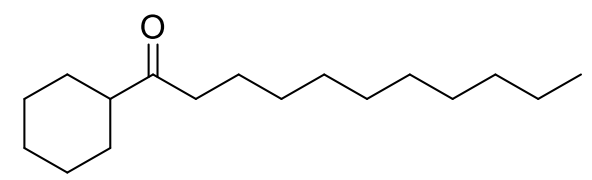

Compound $\mathbf{2 u}$ was prepared as a colorless oil in $97 \%$ yield $(73.3 \mathrm{mg}$, eluent: petroleum ether $/$ EtOAc $=100 / 1$ to 50/1) from 1-cyclohexylundec-10-en-1-ol 1u (75.6 mg, $0.3 \mathrm{mmol})$, [ RhCl(cod) ]2 (3.0 mg, $0.006 \mathrm{mmol})$, Xantphos (6.9 mg, 0.012 $\mathrm{mmol}$ ), and $\mathrm{CsOAc}(2.9 \mathrm{mg}, 0.015 \mathrm{mmol})$ following the above general procedure $\mathrm{C}$. This compound was previously reported. ${ }^{31}$

$\mathrm{R}_{f}=0.90$ (petroleum ether $/$ EtOAc $=10 / 1$ )

${ }^{1} \mathrm{H}$ NMR (400 MHz, $\left.\mathrm{CDCl}_{3}\right)$ \& $2.41(\mathrm{t}, J=7.4 \mathrm{~Hz}, 2 \mathrm{H}), 2.36-2.28(\mathrm{~m}, 1 \mathrm{H}), 1.85-$ $1.73(\mathrm{~m}, 4 \mathrm{H}), 1.59-1.50(\mathrm{~m}, 2 \mathrm{H}), 1.40-1.17(\mathrm{~m}, 20 \mathrm{H}), 0.88(\mathrm{t}, J=6.7 \mathrm{~Hz}, 3 \mathrm{H})$.

${ }^{13} \mathrm{C}$ NMR (100 MHz, $\left.\mathrm{CDCl}_{3}\right) \delta$ 214.6, 51.0, 40.8, 32.0, 29.7, 29.6, 29.6, 29.5, 29.5, 28.7, 26.0, 25.9, 23.9, 22.8, 14.2 .

\section{1-Cyclopentylundecan-1-one (2v).}

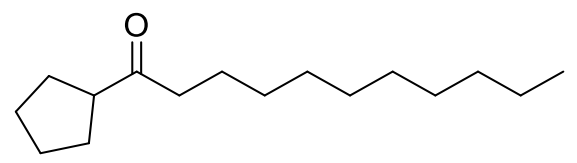

Compound $2 \mathbf{v}$ was prepared as a colorless oil in $98 \%$ yield $(70.0 \mathrm{mg}$, eluent: petroleum ether/EtOAc = 100/1 to 50/1) from 1-cyclopentylundec-10-en-1-ol 1v (71.4 mg, $0.3 \mathrm{mmol})$, [RhCl(cod)]2 (3.0 mg, $0.006 \mathrm{mmol})$, Xantphos (6.9 mg, 0.012 $\mathrm{mmol})$, and $\mathrm{CsOAc}(2.9 \mathrm{mg}, 0.015 \mathrm{mmol})$ following the above general procedure $\mathrm{C}$.

$\mathrm{R}_{f}=0.90$ (petroleum ether/EtOAc $=10 / 1$ )

${ }^{1} \mathrm{H}$ NMR $\left(400 \mathrm{MHz}, \mathrm{CDCl}_{3}\right) \delta$ 2.91-2.78 (m, 1H), $2.41(\mathrm{t}, J=6.1 \mathrm{~Hz}, 2 \mathrm{H}), 1.84-$ $1.48(\mathrm{~m}, 10 \mathrm{H}), 1.37-1.15(\mathrm{~m}, 14 \mathrm{H}), 0.86(\mathrm{t}, J=5.6 \mathrm{~Hz}, 3 \mathrm{H})$.

${ }^{13} \mathrm{C}$ NMR (100 MHz, $\left.\mathrm{CDCl}_{3}\right)$ \& 213.7, 51.5, 42.0, 32.0, 29.7, 29.62, 29.59, 29.5, 29.4, 29.0, 26.1, 24.0, 22.8, 14.2 .

HRMS (ESI') calcd for $\mathrm{C}_{16} \mathrm{H}_{31} \mathrm{O}^{+}[\mathrm{M}+\mathrm{Na}]^{+}:$239.2369, found: 239.2369 .

IR (neat, cm ${ }^{-1}$ ): 2924, 2856, 1710, 1459, 1409, 1370, 1131, 967, 722.

1-Cyclopropylundecan-1-one (2w). 


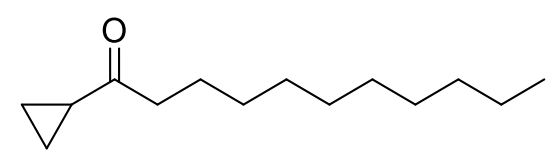

Compound $2 \mathbf{w}$ was prepared as a colorless oil in $82 \%$ yield $(51.7 \mathrm{mg}$, eluent: petroleum ether/EtOAc $=100 / 1$ to 50/1) from 1-cyclopropylundec-10-en-1-ol $1 \mathbf{w}$ (63.0 mg, $0.3 \mathrm{mmol})$, [RhCl(cod)]2 (3.0 mg, $0.006 \mathrm{mmol})$, Xantphos (6.9 mg, 0.012 $\mathrm{mmol})$, and $\mathrm{CsOAc}(2.9 \mathrm{mg}, 0.015 \mathrm{mmol})$ following the above general procedure $\mathrm{C}$.

$\mathrm{R}_{f}=0.88$ (petroleum ether $/$ EtOAc $=10 / 1$ )

${ }^{1} \mathrm{H}$ NMR $\left(400 \mathrm{MHz}, \mathrm{CDCl}_{3}\right) \delta 2.53(\mathrm{t}, J=7.5 \mathrm{~Hz}, 2 \mathrm{H}), 1.96-1.88(\mathrm{~m}, 1 \mathrm{H}), 1.69-$ $1.55(\mathrm{~m}, 2 \mathrm{H}), 1.38-1.21(\mathrm{~m}, 14 \mathrm{H}), 1.04-0.97(\mathrm{~m}, 2 \mathrm{H}), 0.93-0.81(\mathrm{~m}, 5 \mathrm{H})$.

${ }^{13} \mathrm{C}$ NMR (100 MHz, CDCl $)$ $\delta$ 211.5, 43.7, 32.0, 29.7, 29.62, 29.56, 29.5, 29.4, 24.2, 22.8, 20.4, 14.2, 10.7 .

HRMS (ESI+) calcd for $\mathrm{C}_{14} \mathrm{H}_{27} \mathrm{O}^{+}[\mathrm{M}+\mathrm{H}]^{+}:$211.2056, found: 211.2056.

IR (neat, cm ${ }^{-1}$ ): 2923, 2854, 1701, 1460, 1384, 1192, 1083, 1020, 968, 898, 817, 723.

\section{2-Methyltridecan-3-one (2x).}

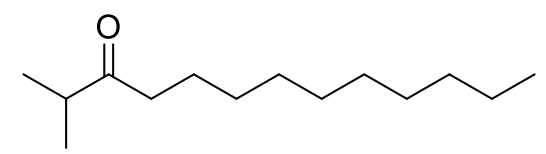

Compound 2x was prepared as a colorless oil in $88 \%$ yield $(56.0 \mathrm{mg}$, eluent: petroleum ether/EtOAc = 100/1 to 50/1) from 2-methyltridec-12-en-3-ol 1x (63.6 $\mathrm{mg}, 0.3 \mathrm{mmol}),[\mathrm{RhCl}(\mathrm{cod})]_{2}(3.0 \mathrm{mg}, 0.006 \mathrm{mmol})$, Xantphos $(6.9 \mathrm{mg}, 0.012$ $\mathrm{mmol})$, and CsOAc $(2.9 \mathrm{mg}, 0.015 \mathrm{mmol})$ following the above general procedure $\mathrm{C}$. This compound was previously reported. ${ }^{31}$

$\mathrm{R}_{f}=0.93$ (petroleum ether $/$ EtOAc $=10 / 1$ )

${ }^{1} \mathrm{H}$ NMR (400 MHz, $\left.\mathrm{CDCl}_{3}\right) \delta$ 2.67-2.53 (m, 1H), $2.43(\mathrm{t}, J=7.4 \mathrm{~Hz}, 2 \mathrm{H}), 1.62$ $1.51(\mathrm{~m}, 2 \mathrm{H}), 1.34-1.20(\mathrm{~m}, 14 \mathrm{H}), 1.09(\mathrm{~s}, 3 \mathrm{H}), 1.08(\mathrm{~s}, 3 \mathrm{H}), 0.88(\mathrm{t}, J=6.8 \mathrm{~Hz}, 3 \mathrm{H})$. ${ }^{13} \mathrm{C}$ NMR (100 MHz, $\left.\mathrm{CDCl}_{3}\right) \delta$ 215.2, 40.9, 40.5, 32.0, 29.7, 29.63, 29.59, 29.5, 29.4, $23.9,22.8,18.4,14.2$.

Tridecan-3-one (2y). 


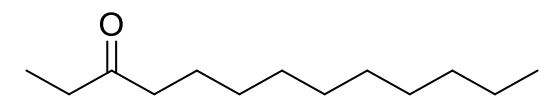

Compound $2 \mathbf{y}$ was prepared as a colorless oil in $94 \%$ yield (55.8 $\mathrm{mg}$, eluent: petroleum ether/EtOAc $=100 / 1$ to 50/1) from tridec-12-en-3-ol 1y (59.4 mg, 0.3 $\mathrm{mmol})$, [RhCl(cod) ]2 (3.0 mg, $0.006 \mathrm{mmol})$, Xantphos $(6.9 \mathrm{mg}, 0.012 \mathrm{mmol})$, and CsOAc $(2.9 \mathrm{mg}, 0.015 \mathrm{mmol})$ following the above general procedure C. This compound was previously reported..$^{14}$

$\mathrm{R}_{f}=0.90$ (petroleum ether/EtOAc $=10 / 1$ )

${ }^{1}$ H NMR (400 MHz, CDCl$\left._{3}\right) \delta$ 2.51-2.34 (m, 4H), 1.63-1.48 (m, 2H), 1.37-1.19 (m, $14 \mathrm{H}), 1.05(\mathrm{t}, J=7.3 \mathrm{~Hz}, 3 \mathrm{H}), 0.88(\mathrm{t}, J=6.8 \mathrm{~Hz}, 3 \mathrm{H})$.

${ }^{13} \mathrm{C}$ NMR (100 MHz, $\left.\mathrm{CDCl}_{3}\right) \delta$ 212.1, 42.6, 35.9, 32.0, 29.7, 29.6, 29.5, 29.42, 29.40,

$24.1,22.8,14.2,7.9$.

\section{Dodecan-2-one (2z).}

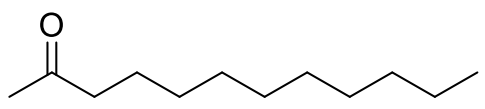

Compound $2 \mathrm{z}$ was prepared as a colorless oil in $70 \%$ yield $(38.6 \mathrm{mg}$, eluent: petroleum ether/EtOAc $=100 / 1$ to 50/1) from dodec-11-en-2-ol 1z $(55.2 \mathrm{mg}, 0.3$ $\mathrm{mmol}),[\mathrm{RhCl}(\mathrm{cod})]_{2}(3.0 \mathrm{mg}, 0.006 \mathrm{mmol})$, Xantphos $(6.9 \mathrm{mg}, 0.012 \mathrm{mmol})$, and CsOAc $(2.9 \mathrm{mg}, 0.015 \mathrm{mmol})$ following the above general procedure C. This compound was previously reported..$^{32}$

$\mathrm{R}_{f}=0.90$ (petroleum ether $/$ EtOAc $\left.=10 / 1\right)$

${ }^{1} \mathrm{H}$ NMR (400 MHz, $\left.\mathrm{CDCl}_{3}\right) \delta 2.42(\mathrm{t}, J=7.5 \mathrm{~Hz}, 2 \mathrm{H}), 2.13(\mathrm{~s}, 3 \mathrm{H}), 1.63-1.50(\mathrm{~m}$, $2 \mathrm{H}), 1.38-1.20(\mathrm{~m}, 14 \mathrm{H}), 0.88(\mathrm{t}, J=6.8 \mathrm{~Hz}, 3 \mathrm{H})$.

${ }^{13} \mathrm{C}$ NMR (100 MHz, $\left.\mathrm{CDCl}_{3}\right) \delta$ 209.5, 43.9, 32.0, 29.9, 29.7, 29.6, 29.5, 29.4, 29.3, $24.0,22.8,14.2$.

3,7-Dimethyloct-6-enal (2aa).

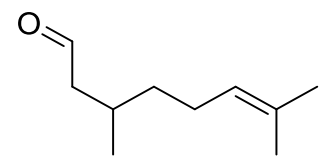

Compound 2aa was prepared as a colorless oil in 57\% yield (26.3 mg, eluent: 
petroleum ether $/ \mathrm{EtOAc}=100 / 1$ to $50 / 1)$ from nerol 1 aa $(46.2 \mathrm{mg}, 0.3 \mathrm{mmol})$, [RhCl(cod)]2 (7.4 mg, $0.015 \mathrm{mmol})$, Xantphos (17.3 mg, $0.03 \mathrm{mmol})$, and CsOAc ( $5.8 \mathrm{mg}, 0.03 \mathrm{mmol}$ ) following the above general procedure $\mathrm{C}$. This compound was previously reported..$^{33}$

$\mathrm{R}_{f}=0.88$ (petroleum ether $/$ EtOAc $=10 / 1$ )

${ }^{1} \mathrm{H}$ NMR (400 MHz, CDCl $) \delta 9.75(\mathrm{~s}, 1 \mathrm{H}), 5.09$ (t, J = 7.3 Hz, 1H), 2.48-2.37 (m, $1 \mathrm{H}), 2.28-2.17(\mathrm{~m}, 1 \mathrm{H}), 2.13-1.94(\mathrm{~m}, 3 \mathrm{H}), 1.69(\mathrm{~s}, 3 \mathrm{H}), 1.60(\mathrm{~s}, 3 \mathrm{H}), 1.43-1.31(\mathrm{~m}$, $1 \mathrm{H}), 1.30-1.21(\mathrm{~m}, 1 \mathrm{H}), 1.03-0.94(\mathrm{~m}, 3 \mathrm{H})$.

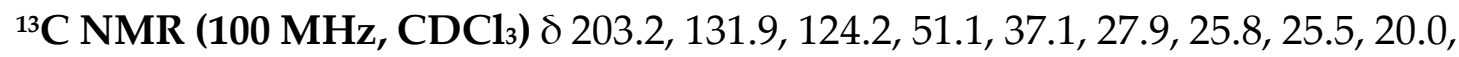
17.8.

$2 \mathrm{ab}$

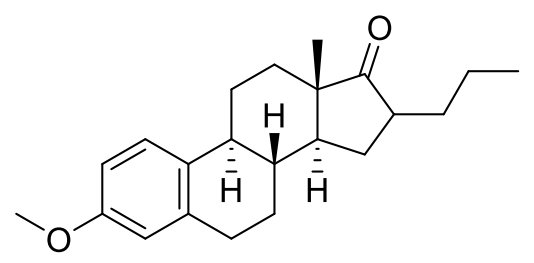

Compound 2ab was prepared as a colorless oil in $64 \%$ yield $(63.1 \mathrm{mg}$, eluent: petroleum ether/EtOAc $=100 / 1$ to $50 / 1)$ from $1 \mathrm{ab}(98.1 \mathrm{mg}, 0.3 \mathrm{mmol}$ ), $[\mathrm{RhCl}(\mathrm{cod})]_{2}(3.0 \mathrm{mg}, 0.006 \mathrm{mmol})$, Xantphos $(6.9 \mathrm{mg}, 0.012 \mathrm{mmol})$, and CsOAc ( $2.9 \mathrm{mg}, 0.015 \mathrm{mmol}$ ) following the above general procedure $\mathrm{C}$.

$\mathrm{R}_{f}=0.61$ (petroleum ether $/$ EtOAc $=10 / 1$ )

${ }^{1} \mathrm{H}$ NMR $\left(400 \mathrm{MHz}, \mathrm{CDCl}_{3}\right) \delta 7.20(\mathrm{~d}, J=8.6 \mathrm{~Hz}, 1 \mathrm{H}), 6.71(\mathrm{~d}, J=8.5 \mathrm{~Hz}, 1 \mathrm{H})$, $6.64(\mathrm{~s}, 1 \mathrm{H}), 3.77(\mathrm{~s}, 3 \mathrm{H}), 2.96-2.84(\mathrm{~m}, 2 \mathrm{H}), 2.43-2.33(\mathrm{~m}, 1 \mathrm{H}), 2.32-2.18(\mathrm{~m}, 2 \mathrm{H})$, 2.16-2.08 (m, 1H), 2.09-1.92 (m, 2H), 1.90-1.80 (m, 1H), 1.62-1.25 (m, 9H), 0.97$0.90(\mathrm{~m}, 3 \mathrm{H}), 0.88-0.82(\mathrm{~m}, 3 \mathrm{H})$.

${ }^{13} \mathrm{C}$ NMR (100 MHz, CDCl $)$ $) \delta 223.0,157.7,137.8,132.2,126.4,114.0,111.6,55.3$, 49.3, 49.1, 48.5, 44.2, 38.1, 34.7, 32.1, 29.8, 28.7, 26.8, 26.0, 21.4, 14.1, 14.0.

HRMS (EI+) calcd for $\mathrm{C}_{22} \mathrm{H}_{30} \mathrm{O}_{2}^{+}\left[\mathrm{M}^{+}\right]$: 326.2240, found: 326.2242 .

IR (neat, cm ${ }^{-1}$ ): 2929, 2867, 1735, 1610, 1500, 1459, 1311, 1281, 1254, 1157, 1045, 817.

\section{$2 \mathrm{ac}$}




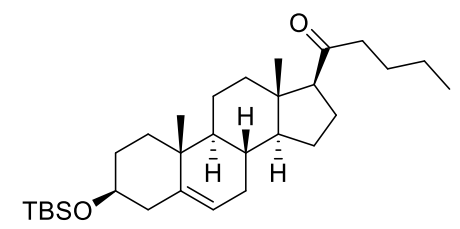

Compound 2ac was prepared as a colorless oil in $86 \%$ yield $(121.8 \mathrm{mg}$, eluent: petroleum ether $/$ EtOAc $=100 / 1$ to $50 / 1)$ from 1 ac $(141.6 \mathrm{mg}, 0.3 \mathrm{mmol})$, $[\mathrm{RhCl}(\mathrm{cod})]_{2}(7.4 \mathrm{mg}, 0.015 \mathrm{mmol})$, Xantphos (17.3 mg, $\left.0.03 \mathrm{mmol}\right)$, and CsOAc (5.8 $\mathrm{mg}, 0.03 \mathrm{mmol}$ ) following the above general procedure $\mathrm{C}$.

$\mathrm{R}_{f}=0.81$ (petroleum ether $/$ EtOAc $=10 / 1$ )

${ }^{1} \mathrm{H}$ NMR (400 MHz, $\left.\mathrm{CDCl}_{3}\right) \delta 5.30$ (d, J = 5.0 Hz, 1H), 3.53-3.42 (m, 1H), 2.51 (t, $J=8.8 \mathrm{~Hz}, 1 \mathrm{H}), 2.36(\mathrm{t}, J=7.4 \mathrm{~Hz}, 2 \mathrm{H}), 2.30-2.21(\mathrm{~m}, 1 \mathrm{H}), 2.20-2.12(\mathrm{~m}, 2 \mathrm{H}), 2.04-$ $1.95(\mathrm{~m}, 2 \mathrm{H}), 1.86-1.77(\mathrm{~m}, 1 \mathrm{H}), 1.74-1.37$ (m, 12H), 1.34-1.06 (m, 5H), 0.99 (s, $3 \mathrm{H}), 0.92-0.84(\mathrm{~m}, 12 \mathrm{H}), 0.60(\mathrm{~s}, 3 \mathrm{H}), 0.05(\mathrm{~s}, 6 \mathrm{H})$.

${ }^{13} \mathrm{C}$ NMR (100 MHz, $\mathrm{CDCl}_{3}$ ) $\delta$ 211.9, 141.7, 121.0, 72.7, 63.0, 57.2, 50.2, 44.3, 44.2, $42.9,39.1,37.5,36.7,32.2,32.01,31.98,26.1,26.0,24.7,23.1,22.6,21.2,19.6,18.4$, $14.1,13.5,-4.4$.

HRMS (ESI') calcd for $\mathrm{C}_{30} \mathrm{H}_{53} \mathrm{O}_{2} \mathrm{Si}^{+}[\mathrm{M}+\mathrm{Na}]^{+}: 473.3809$, found: 473.3808 .

IR (neat, cm-1): 2932, 2891, 2857, 1707, 1464, 1380, 1261, 1085, 888, 870, 837, 803, $774,733,704$.

2ad

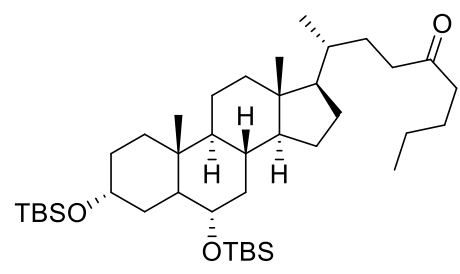

Compound 2ad was prepared as a colorless oil in 90\% yield (178.2 mg, eluent: petroleum ether $/$ EtOAc $=100 / 1$ to $50 / 1)$ from 1 ad $(198.0 \mathrm{mg}, 0.3 \mathrm{mmol})$, $[\mathrm{RhCl}(\mathrm{cod})]_{2}(3.0 \mathrm{mg}, 0.006 \mathrm{mmol})$, Xantphos (6.9 mg, $\left.0.012 \mathrm{mmol}\right)$, and CsOAc $(2.9 \mathrm{mg}, 0.015 \mathrm{mmol})$ following the above general procedure $\mathrm{C}$.

$\mathrm{R}_{f}=0.72$ (petroleum ether $/$ EtOAc $=10 / 1$ )

${ }^{1} \mathrm{H}$ NMR (400 MHz, $\left.\mathrm{CDCl}_{3}\right) \delta$ 4.03-3.91 (m, 1H), 3.59-3.44 (m, 1H), 2.45-2.34 (m, $3 \mathrm{H}), 2.33-2.23(\mathrm{~m}, 1 \mathrm{H}), 1.97-1.78(\mathrm{~m}, 3 \mathrm{H}), 1.76-1.65(\mathrm{~m}, 2 \mathrm{H}), 1.62-1.48(\mathrm{~m}, 4 \mathrm{H})$, 
1.47-1.33 (m, 8H), 1.32-1.21 (m, 5H), 1.18-0.98 (m, 6H), 0.92-0.79 (m, 27H), 0.60 $(\mathrm{s}, 3 \mathrm{H}), 0.03(\mathrm{~s}, 6 \mathrm{H}), 0.01(\mathrm{~s}, 6 \mathrm{H})$.

${ }^{13} \mathrm{C}$ NMR (100 MHz, $\left.\mathrm{CDCl}_{3}\right) \delta$ 212.1, 73.0, 68.7, 56.2, 56.1, 49.7, 42.9, 42.6, 40.1, 39.7, 36.1, 36.0, 35.5, 35.4, 35.0, 31.1, 29.9, 28.2, 26.1 (double), 26.0 (double), 24.3, 23.6, 22.5, 20.9, 18.5, 18.2, 14.0, 12.2, $-4.4,-4.5,-4.6,-4.7$.

HRMS (ESI ${ }^{+}$) calcd for $\mathrm{C}_{40} \mathrm{H}_{76} \mathrm{NaO}_{3} \mathrm{Si}_{2}{ }^{+}[\mathrm{M}+\mathrm{Na}]^{+}:$683.5225, found: 683.5221 .

IR (neat, cm-1): 2931, 2858, 1716, 1465, 1376, 1252, 1079, 1006, 926, 859, 836, 775, 669.

\section{Gram-Scale Reaction.}

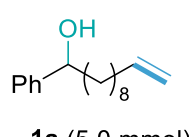

1 a $(5.0 \mathrm{mmol})$
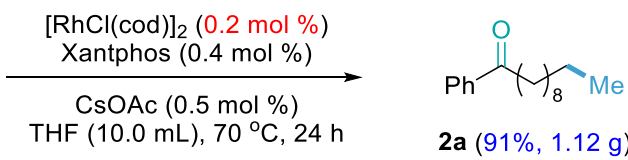

2a $(91 \%, 1.12 \mathrm{~g})$

In glove box, to an oven-dried 25-mL round bottom flask equipped with a magnetic stir bar were added $[\mathrm{RhCl}(\mathrm{cod})]_{2}(4.9 \mathrm{mg}, 0.01 \mathrm{mmol})$, Xantphos (11.6 $\mathrm{mg}, 0.02 \mathrm{mmol})$, and anhydrous THF $(5.0 \mathrm{~mL})$. The resulting mixture was stirred for $10 \mathrm{~min}$ at room temperature, then CsOAc $(4.8 \mathrm{mg}, 0.025 \mathrm{mmol})$ was added. The resulting mixture was stirred for an additional $10 \mathrm{~min}$, then $\mathbf{1 a}(1.23$ g, $5.00 \mathrm{mmol})$ in THF $(5.0 \mathrm{~mL})$ was added. The vial was sealed with a reflux condenser, and the mixture was stirred at $70{ }^{\circ} \mathrm{C}$ in an oil bath for $24 \mathrm{~h}$. The reaction mixture was filtered through celite, and washed with EtOAc. The filtrate was concentrated in vacuo, and the residue was purified by silica gel chromatography (petroleum ether/EtOAc $=20 / 1$ ) to afford the desired ketone 2a (1.12 g, 91\% yield).

\section{Mechanism Studies}

\section{Experiment A}

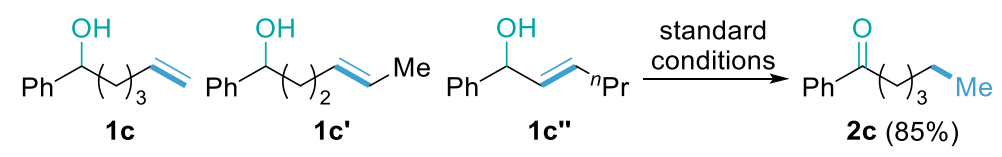


Compound 2c was prepared as a colorless oil in $85 \%$ yield (43.8 mg, eluent: petroleum ether/EtOAc $=100 / 1$ to 50/1) from 1-phenylhex-5-en-1-ol 1c, $(E)-1$ -

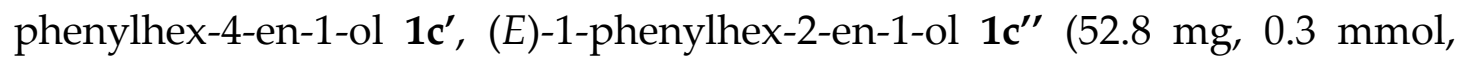
1/1/1), [RhCl(cod)]2 (3.0 mg, $0.006 \mathrm{mmol})$, Xantphos (6.9 mg, $0.012 \mathrm{mmol})$, and CsOAc (2.9 mg, $0.015 \mathrm{mmol})$ following the above general procedure $\mathrm{C}$.

\section{Experiment B}

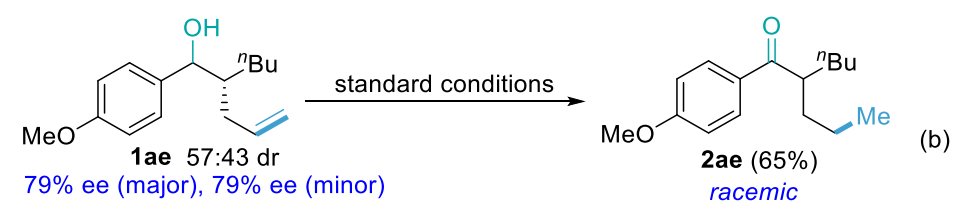

Compound 2ae was prepared as a colorless oil in 65\% yield (37.0 mg, eluent: petroleum ether/EtOAc $=50 / 1$ to 20/1) from (2R)-2-allyl-1-(4methoxyphenyl)hexan-1-ol 1ae $(56.7 \mathrm{mg}, 0.23 \mathrm{mmol})$, [ $\mathrm{RhCl}(\mathrm{cod})]_{2}(2.3 \mathrm{mg}$, $0.0046 \mathrm{mmol})$, Xantphos (5.3 mg, $0.0092 \mathrm{mmol})$, and CsOAc (2.2 mg, 0.0116 $\mathrm{mmol}$ ) following the above general procedure $\mathrm{C}$.

$\mathrm{R}_{f}=0.66$ (petroleum ether $/$ EtOAc $=10 / 1$ )

${ }^{1} \mathrm{H}$ NMR (400 MHz, $\left.\mathrm{CDCl}_{3}\right) \delta 7.96(\mathrm{~d}, J=8.1 \mathrm{~Hz}, 2 \mathrm{H}), 6.95(\mathrm{~d}, J=8.1 \mathrm{~Hz}, 2 \mathrm{H})$, $3.87(\mathrm{~s}, 3 \mathrm{H}), 3.46-3.34(\mathrm{~m}, 1 \mathrm{H}), 1.81-1.67$ (m, 2H), 1.56-1.41 (m, 2H), 1.36-1.18 (m, 6H), 0.93-0.80 (m, 6H).

${ }^{13} \mathrm{C}$ NMR (100 MHz, $\left.\mathrm{CDCl}_{3}\right) \delta$ 203.5, 163.4, 131.0, 130.6, 113.9, 55.6, 45.6, 35.1, 32.6, 30.0, 23.1, 21.0, 14.4, 14.1.

HRMS (ESI') calcd for $\mathrm{C}_{16} \mathrm{H}_{24} \mathrm{NaO}_{2}^{+}\left[\mathrm{M}+\mathrm{Na}^{+}\right]$: 271.1669, found: 271.1669.

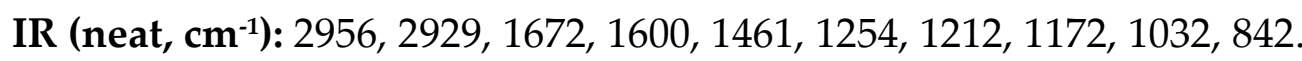

Enantiomeric excess was determined by chiral HPLC (Daicel Chiralpak IC, hexane/isopropyl alcohol $=98.5 / 1.5$, flow rate $=0.5 \mathrm{~mL} / \mathrm{min}, \lambda=254 \mathrm{~nm}), t^{1}=$ $14.2 \mathrm{~min}, t^{2}=14.8 \mathrm{~min}$.

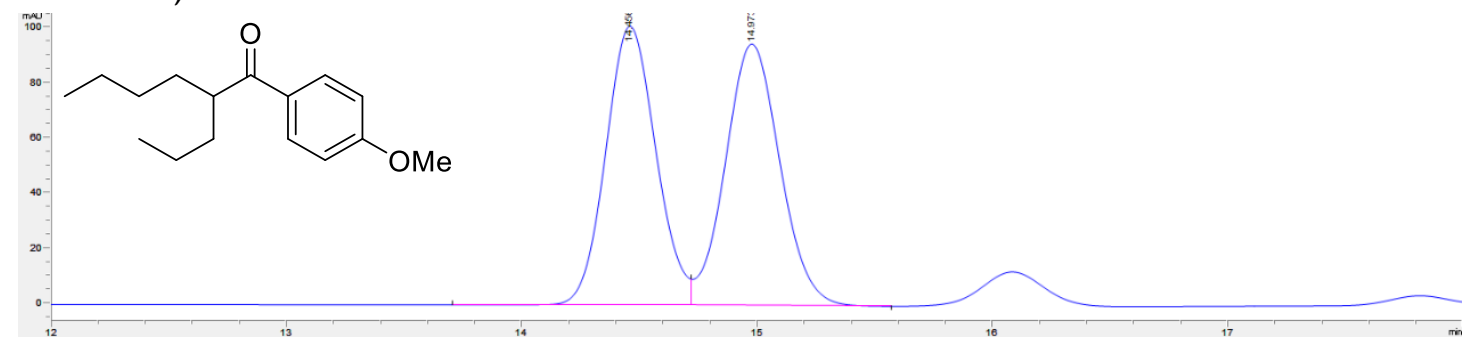


Signal 1: VWD1 $\mathrm{A}$, Wavelength $=254 \mathrm{~nm}$
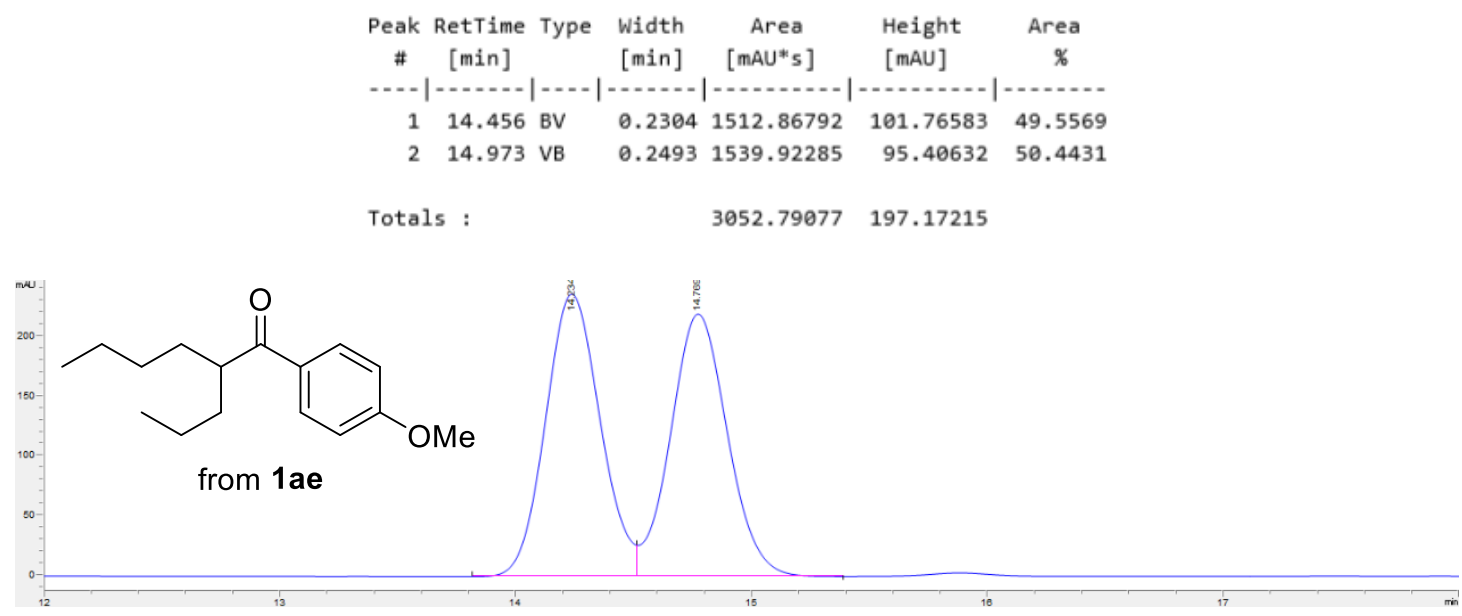

Signal 1: VWD1 A, Wavelength $=254 \mathrm{~nm}$

\begin{tabular}{|c|c|c|c|c|c|c|}
\hline $\begin{array}{c}\text { Peak } \\
\#\end{array}$ & $\begin{array}{c}\text { RetTime } \\
\text { [min] }\end{array}$ & Type & $\begin{array}{l}\text { Width } \\
\text { [min] }\end{array}$ & $\begin{array}{c}\text { Area } \\
\text { [mAU*s] }\end{array}$ & $\begin{array}{l}\text { Height } \\
\text { [mAU] }\end{array}$ & $\begin{array}{c}\text { Area } \\
\%\end{array}$ \\
\hline 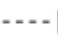 & & & 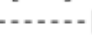 & $\ldots$ & . & . \\
\hline 1 & 14.234 & BV & 0.2500 & 3817.63965 & 236.88434 & 50.9097 \\
\hline 2 & 14.769 & VB & 0.2581 & 3681.20239 & 220.11850 & 49.0903 \\
\hline Tota & : & & & 7498.84204 & 457.00284 & \\
\hline
\end{tabular}

\section{Experiment C}

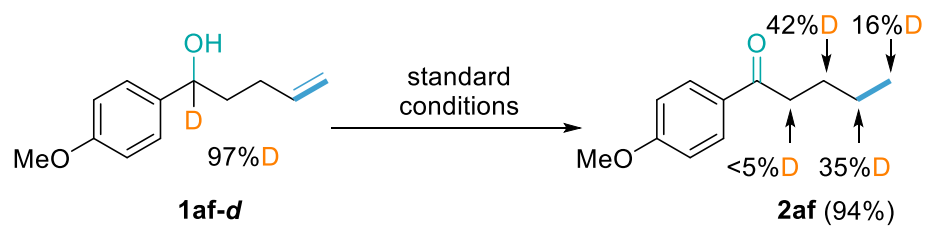

Compound 2af was prepared as a colorless oil in $94 \%$ yield $(54.1 \mathrm{mg}$, eluent: petroleum ether/EtOAc $=50 / 1)$ from 1 af $-d(57.9 \mathrm{mg}, 0.3 \mathrm{mmol}),[\mathrm{RhCl}(\operatorname{cod})]_{2}(3.0$ mg, $0.006 \mathrm{mmol})$, Xantphos (6.9 mg, $0.012 \mathrm{mmol}$ ) and CsOAc (2.9 mg, 0.015 $\mathrm{mmol}$ ) following the above general procedure $\mathrm{C}$. 

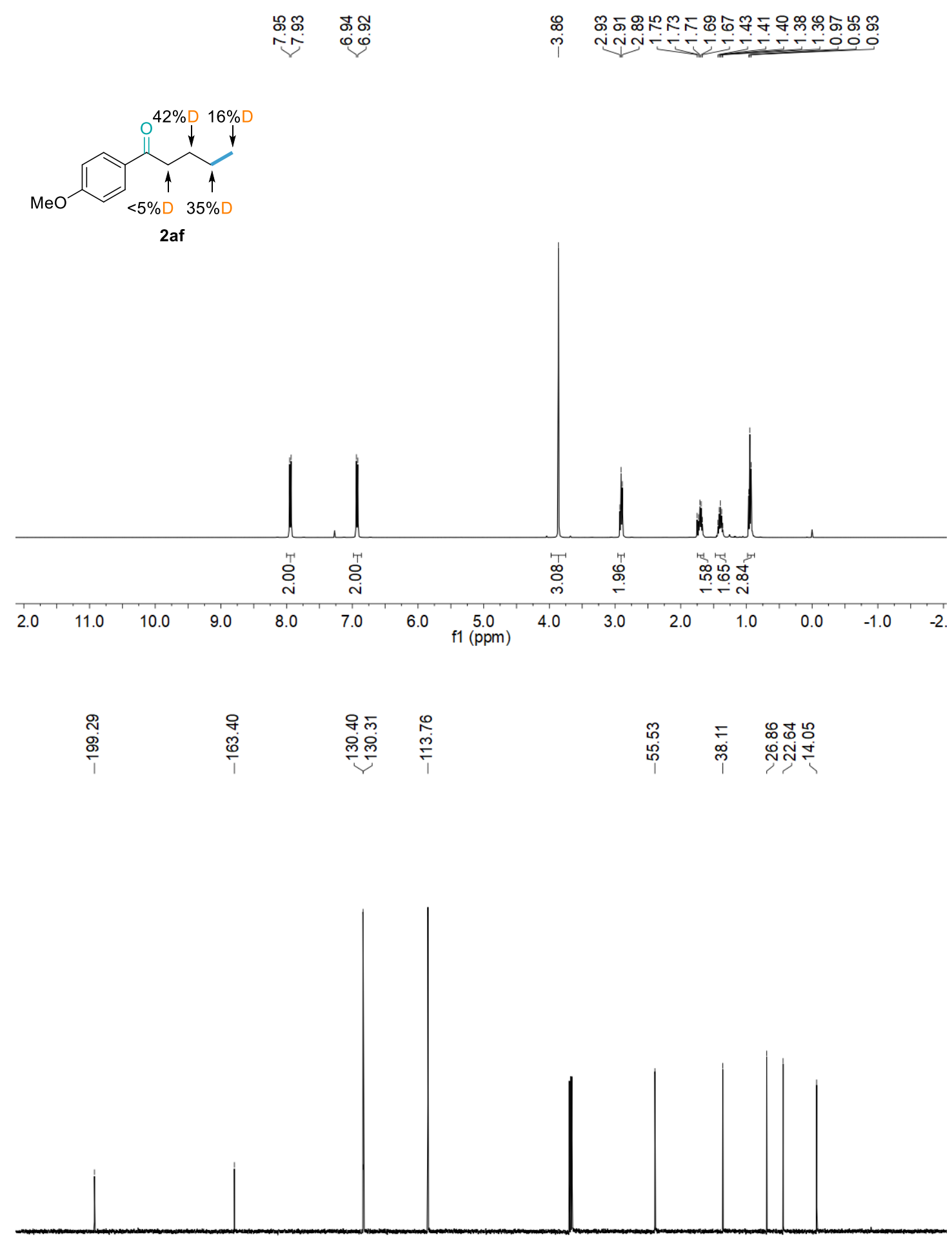

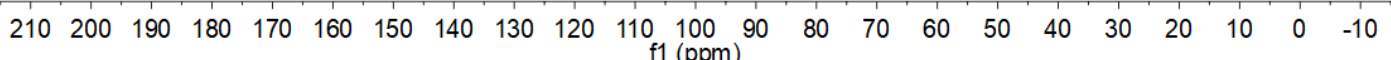

Figure 1. ${ }^{1} \mathrm{H}$ NMR and ${ }^{13} \mathrm{C}$ NMR of 2af.

\section{Experiment D}




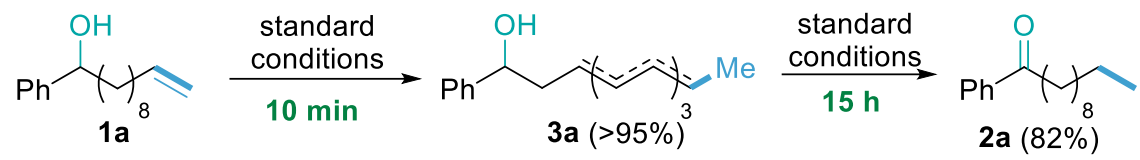

Compound 3a was prepared as a colorless oil in $95 \%$ yield $(73.0 \mathrm{mg}$, eluent: petroleum ether/EtOAc = 10/1) from 1-phenylundec-10-en-1-ol 1a $(73.8 \mathrm{mg}, 0.3$ mmol), [RhCl(cod)]2 (3.0 mg, $0.006 \mathrm{mmol})$, Xantphos (6.9 mg, $0.012 \mathrm{mmol})$ and CsOAc (2.9 mg, $0.015 \mathrm{mmol})$ following the above general procedure $\mathrm{C}$ for 10 $\min$.
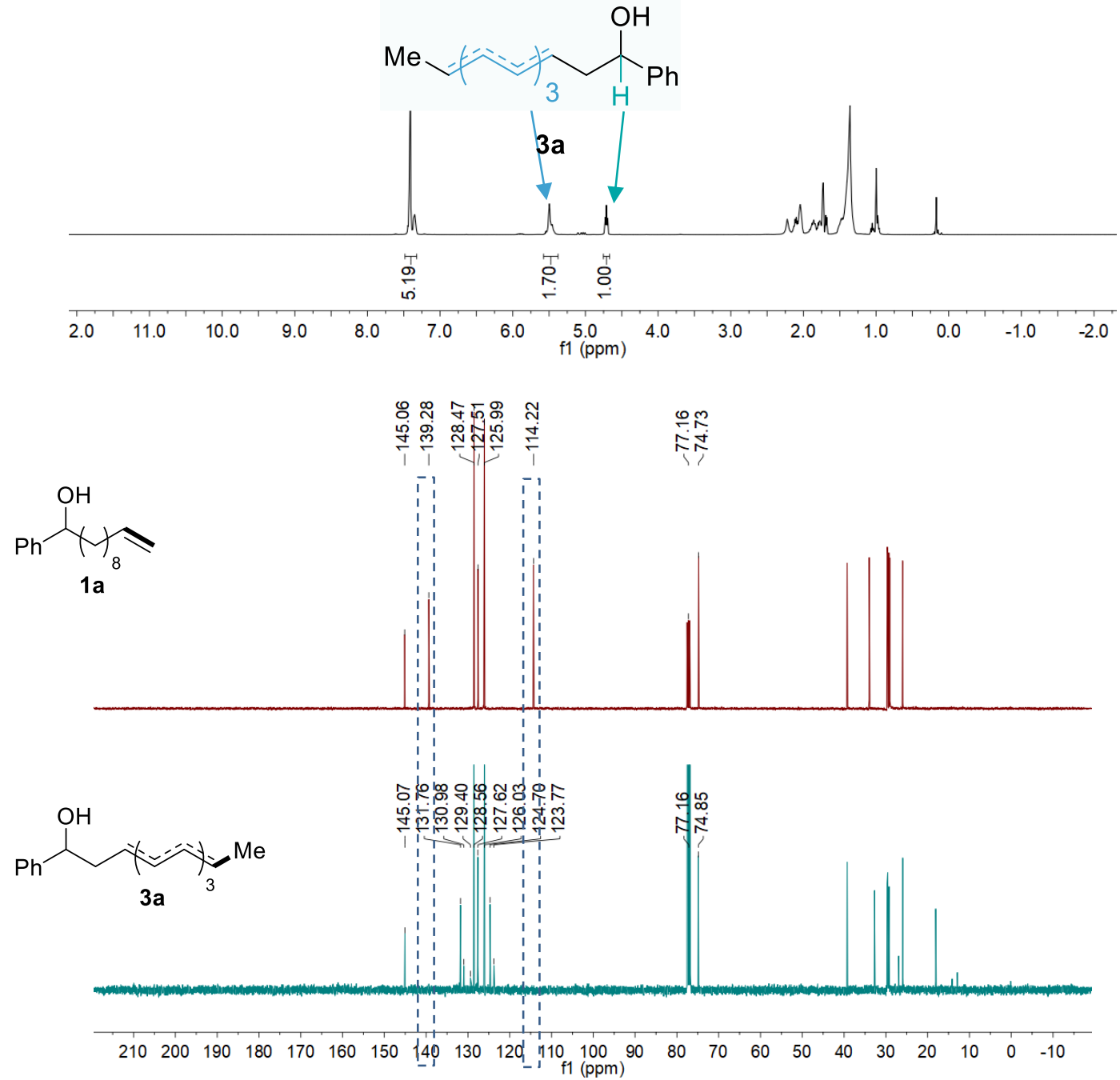

Figure 2. ${ }^{1} \mathrm{H}$ NMR and ${ }^{13} \mathrm{C}$ NMR of $3 a$. 
Compound 2a was prepared as a colorless oil in $82 \%$ yield $(59.9 \mathrm{mg}$, eluent: petroleum ether $/ \mathrm{EtOAc}=50 / 1)$ from $3 \mathbf{a}(73.0 \mathrm{mg}, 0.3 \mathrm{mmol}),[\mathrm{RhCl}(\operatorname{cod})]_{2}(3.0$ $\mathrm{mg}, 0.006 \mathrm{mmol})$, Xantphos (6.9 mg, $0.012 \mathrm{mmol})$ and CsOAc (2.9 mg, 0.015 $\mathrm{mmol}$ ) following the above general procedure $\mathrm{C}$.

\section{Experiment E}<smiles>C=CCC(C)(C)c1ccccc1</smiles>

$1 \mathrm{a}$

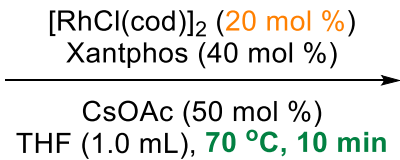

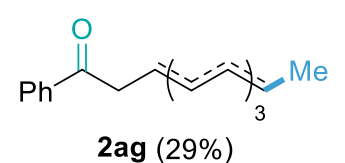

Compound 2ag was prepared as a colorless oil in 29\% yield (7.1 mg, eluent: petroleum ether $/ \mathrm{EtOAc}=50 / 1)$ from $1 \mathrm{a}(24.6 \mathrm{mg}, 0.1 \mathrm{mmol}),[\mathrm{RhCl}(\mathrm{cod})]_{2}(9.9$ $\mathrm{mg}$, $0.02 \mathrm{mmol})$, Xantphos (23.1 mg, $0.04 \mathrm{mmol})$ and CsOAc (9.6 mg, $0.05 \mathrm{mmol})$ following the above general procedure $\mathrm{C}$ for $10 \mathrm{~min}$.
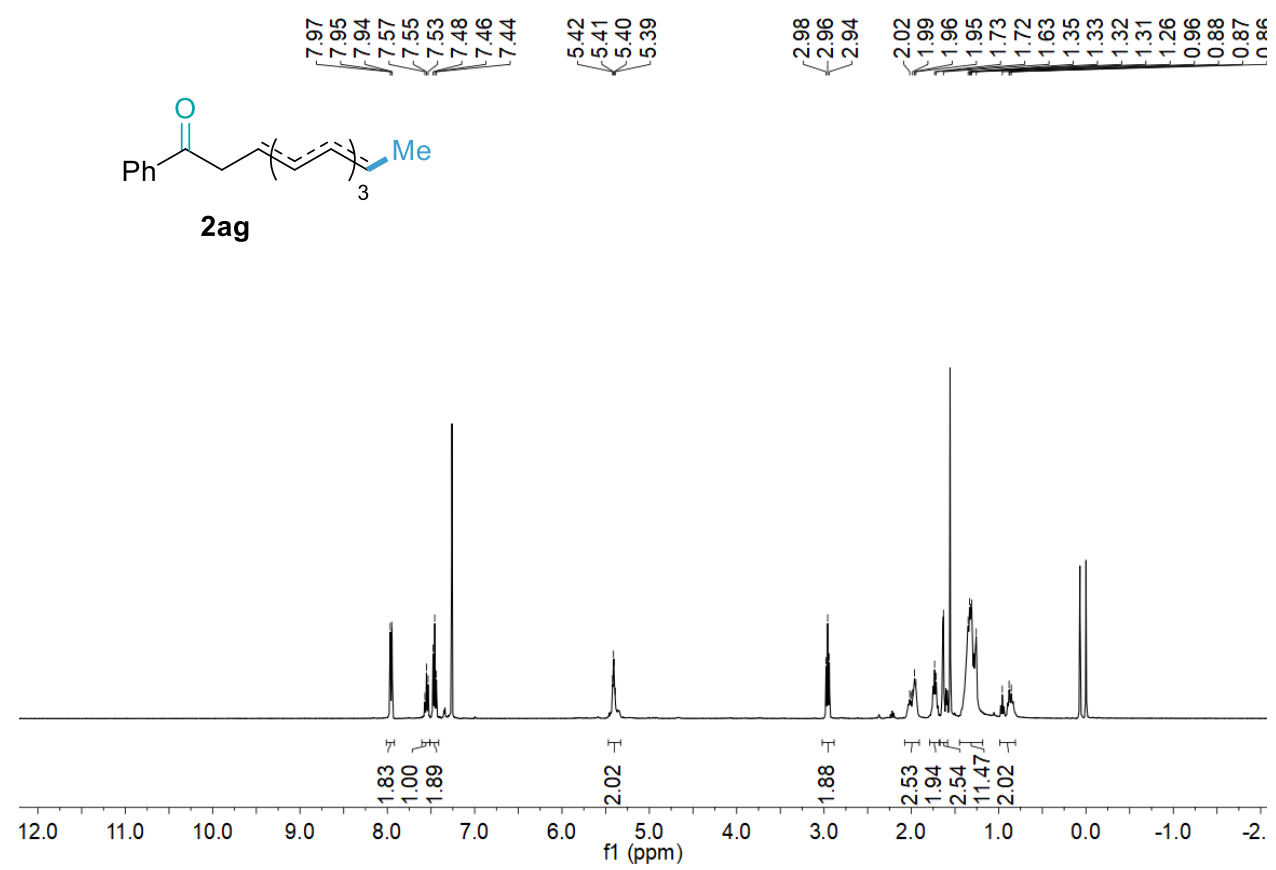


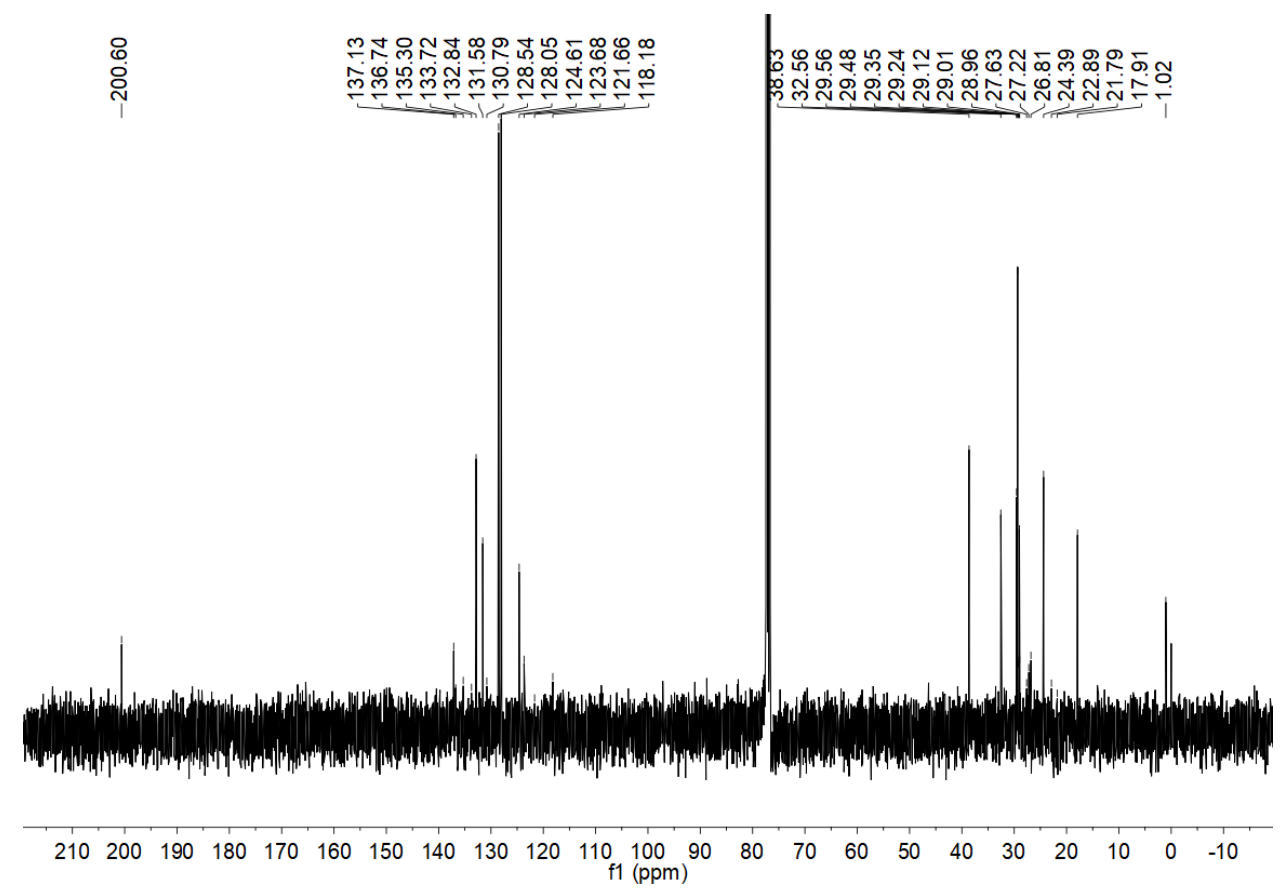

Figure 3. ${ }^{1} \mathrm{H}$ NMR and ${ }^{13} \mathrm{C}$ NMR of 2 ag.

\section{Experiment $\mathrm{F}$}
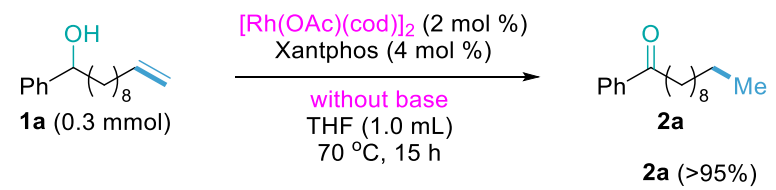

Compound 2a was prepared as a colorless oil in 95\% ${ }^{1} \mathrm{H}$ NMR yield from 1a (108.0 mg, $0.3 \mathrm{mmol})$, [RhOAc(cod)]2 (3.2 mg, $0.006 \mathrm{mmol})$, Xantphos (6.9 mg, $0.012 \mathrm{mmol}$ ) following the above general procedure $\mathrm{C}$.

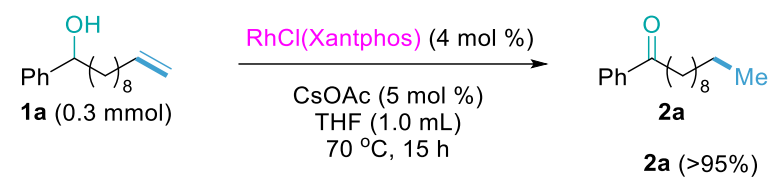

Compound 2a was prepared as a colorless oil in 95\% ${ }^{1} \mathrm{H}$ NMR yield from 1a (108.0 mg, $0.3 \mathrm{mmol}), \mathrm{RhCl}($ Xantphos) $(8.6 \mathrm{mg}, 0.012 \mathrm{mmol})$ and CsOAc (2.9 mg, $0.015 \mathrm{mmol}$ ) following the above general procedure $\mathrm{C}$.

\section{Experiment G}




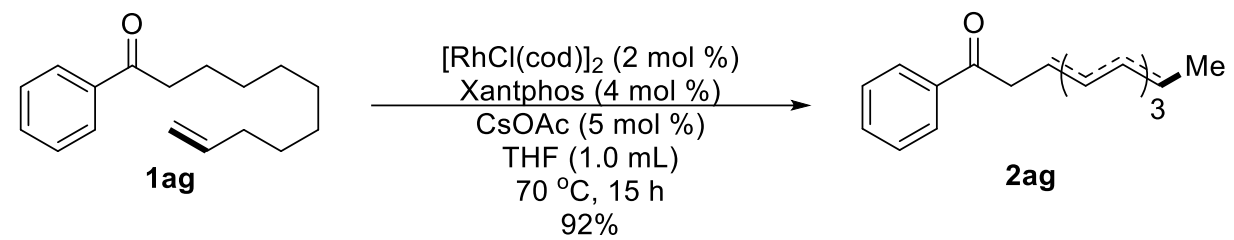

Compound 2ag was prepared as a colorless oil in $92 \%$ yield $(67.3 \mathrm{mg}$, eluent: petroleum ether/EtOAc $=50 / 1)$ from 1-phenylundec-10-en-1-one $1 \mathrm{ag}(73.2 \mathrm{mg}$, $0.3 \mathrm{mmol}),[\mathrm{RhCl}(\mathrm{cod})]_{2}(3.0 \mathrm{mg}, 0.006 \mathrm{mmol})$, Xantphos $(6.9 \mathrm{mg}, 0.012 \mathrm{mmol})$ and $\mathrm{CsOAc}(2.9 \mathrm{mg}, 0.015 \mathrm{mmol})$ following the above general procedure $\mathrm{C}$.
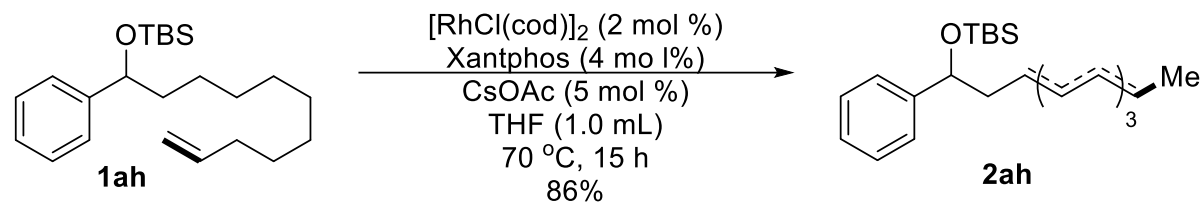

Compound 2ah was prepared as a colorless oil in $86 \%$ yield $(92.9 \mathrm{mg}$, eluent: petroleum ether $/$ EtOAc $=50 / 1)$ from tert-butyldimethyl $((1$-phenylundec-10-en1-yl)oxy)silane 1ah (108.0 mg, $0.3 \mathrm{mmol})$, [RhCl(cod)]2 (3.0 mg, $0.006 \mathrm{mmol})$, Xantphos (6.9 mg, $0.012 \mathrm{mmol})$ and CsOAc $(2.9 \mathrm{mg}, 0.015 \mathrm{mmol})$ following the above general procedure $\mathrm{C}$.

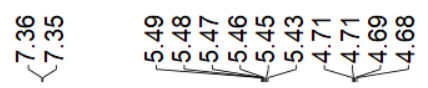

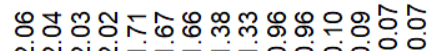

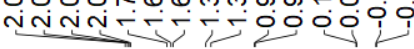

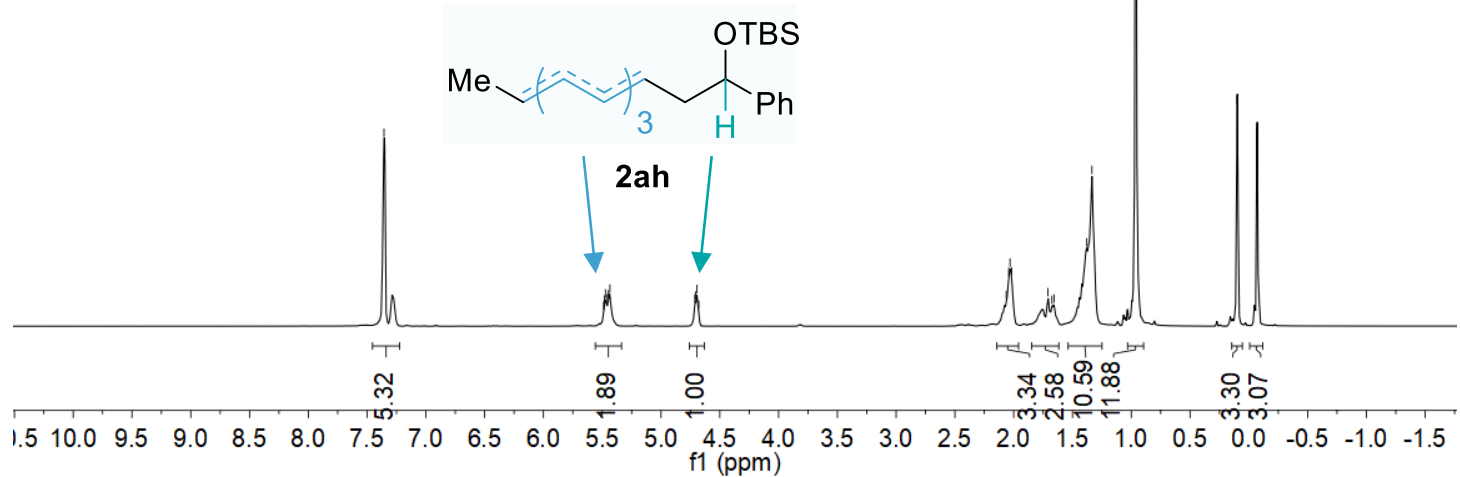

Figure 4. ${ }^{1} \mathrm{H}$ NMR of $2 \mathrm{ah}$. 

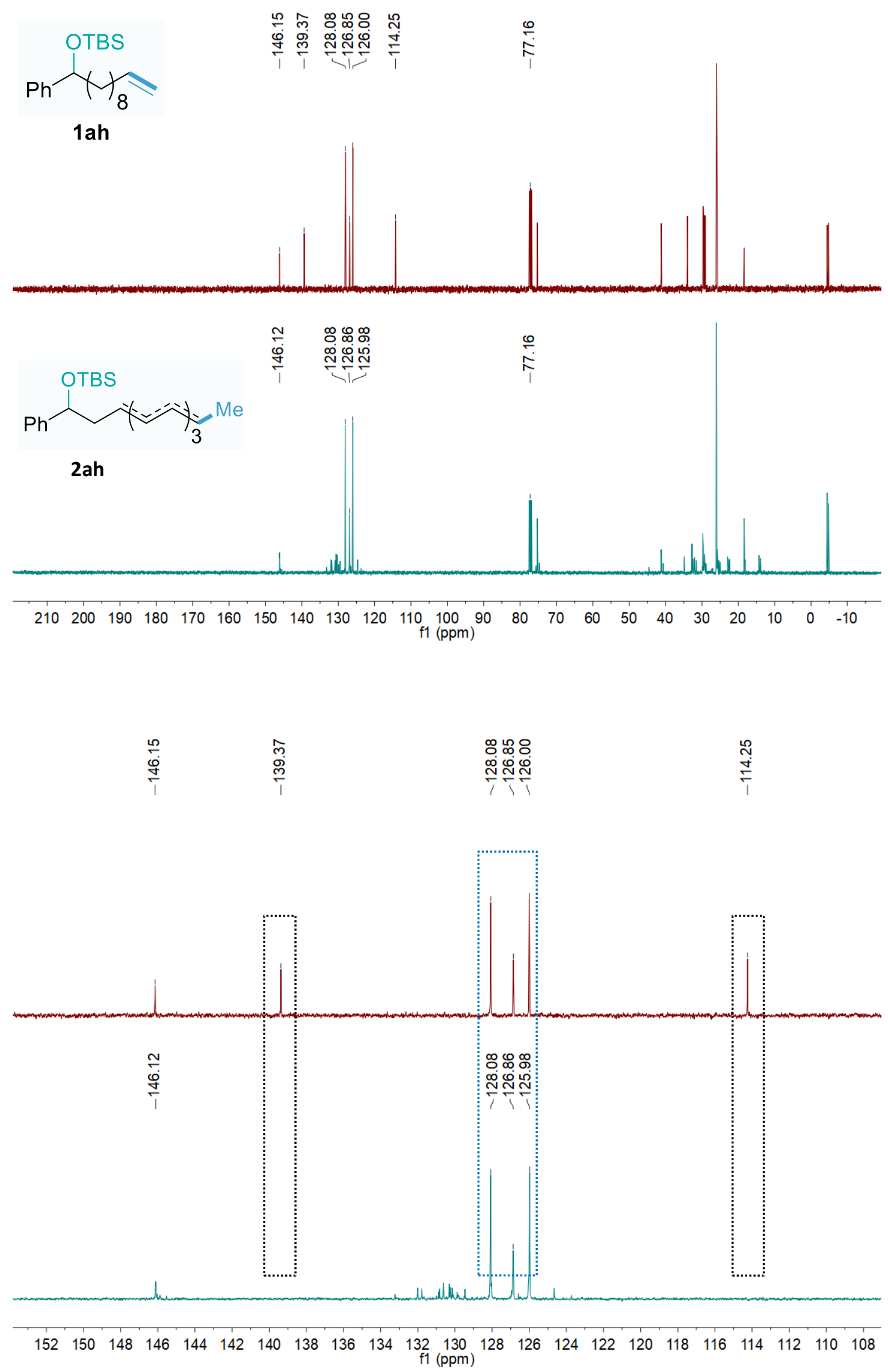

Figure 5. ${ }^{1} \mathrm{H}$ NMR and ${ }^{13} \mathrm{C}$ NMR of $\mathbf{1 a h}$ and $\mathbf{2 a h}$. 


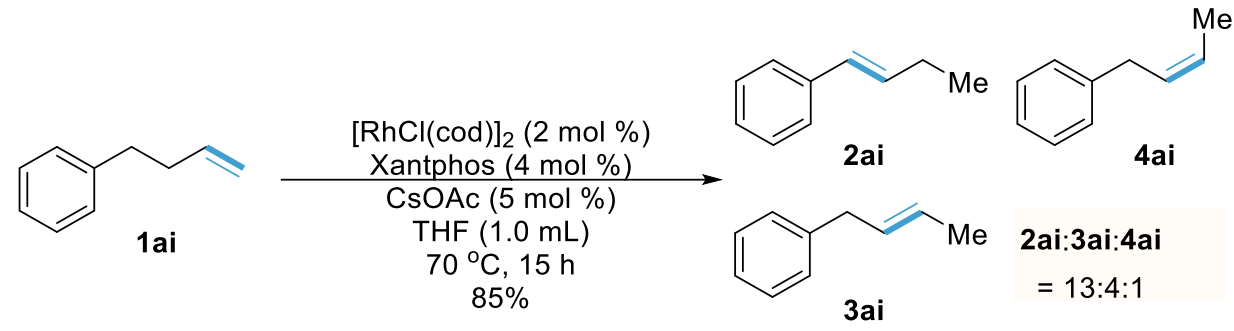

2ai, 3ai and 4ai were prepared as a colorless oil in $85 \%$ yield $(33.7 \mathrm{mg}$, eluent: petroleum ether) from but-3-en-1-ylbenzene 1ai (39.6 mg, $0.3 \mathrm{mmol}$ ), $[\mathrm{RhCl}(\mathrm{cod})]_{2}(3.0 \mathrm{mg}, 0.006 \mathrm{mmol})$, Xantphos $(6.9 \mathrm{mg}, 0.012 \mathrm{mmol})$ and CsOAc ( $2.9 \mathrm{mg}, 0.015 \mathrm{mmol}$ ) following the above general procedure C. Chemical shift data of 2ai, 3ai and 4ai are in accordance with the literature. ${ }^{34}$

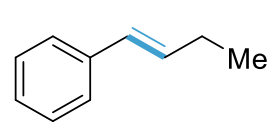

2ai

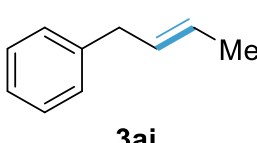

3ai

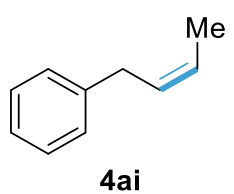

4ai

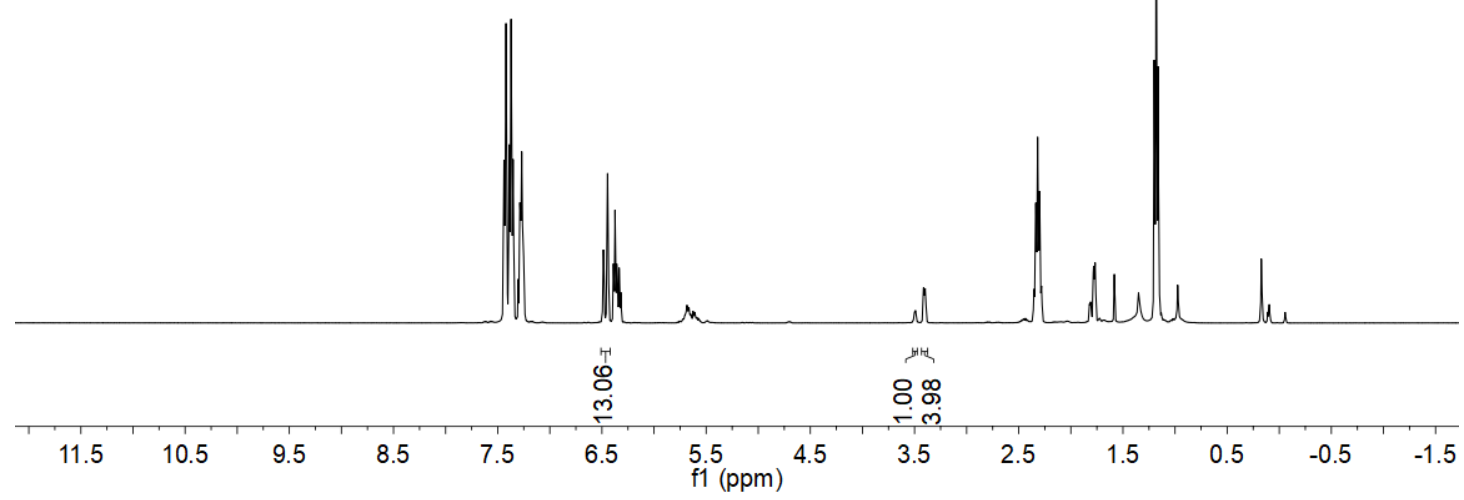

Figure 6. ${ }^{1} \mathrm{H}$ NMR of 2ai, 3ai, and 4ai. 


\section{References}

1. Giordano, G.; Crabtree, R. H.; Heintz, R. M.; Forster, D.; Morris, D. E. Inorg. Synth. 1990, 28,88 .

2. Esteruelas, M. A.; Oliván, M.; Vélez, A. Inorg. Chem. 2013, 52, 5339-5349.

3. Romano, C.; Mazet, C. J. Am. Chem. Soc. 2018, 140, 4743-4750.

4. Lee, C.-H. A.; Loh, T.-P. Tetrahedron Lett. 2004, 45, 5819-5822.

5. Hyugano, T.; Liu, S.; Ouchi, A. J. Org. Chem. 2008, 73, 8861-8866.

6. Pinna, G.; Bellucci, M. C.; Malpezzi, L.; Pisani, L.; Superchi, S.; Volonterio, A.; Zanda, M. Tetrahedron 2011, 67, 5268-5281.

7. Marco, J. A.; Carda, M.; Rodríguez, S.; Castillo, E.; Kneeteman, M. a. N. Tetrahedron 2003, 59, 4085-4101.

8. Yamamoto, H.; Ho, E.; Sasaki, I.; Mitsutake, M.; Takagi, Y.; Imagawa, H.; Nishizawa, M. Eur. J. Org. Chem. 2011, 2011, 2417-2420.

9. Kimura, M.; Ezoe, A.; Mori, M.; Iwata, K.; Tamaru, Y. J. Am. Chem. Soc. 2006, 128, 8559-8568.

10. Nguyen, M. H.; O’Brien, K. T.; Smith, A. B. J. Org. Chem. 2017, 82, 11056-11071.

11. Liu, W.-H.; Wu, H.-J. J. Chin. Chem. Soc. 1988, 35, 241-246.

12. Jeon, J.; Ryu, H.; Lee, C.; Cho, D.; Baik, M.-H.; Hong, S. J. Am. Chem. Soc. 2019, 141, 1004810059 .

13. Schmidt, B. J. Org. Chem. 2004, 69, 7672-7687.

14. Cryle, M. J.; Matovic, N. J.; De Voss, J. J. Org. Lett. 2003, 5, 3341-3344.

15. ten Brink, G.-J.; Arends, I. W. C. E.; Sheldon, R. A. Adv. Synth. Catal. 2002, 344, 355-369.

16. Johnston, J. B.; Singh, A. A.; Clary, A. A.; Chen, C.-K.; Hayes, P. Y.; Chow, S.; De Voss, J. J.; Ortiz de Montellano, P. R. Bioorg. Med. Chem. 2012, 20, 4064-4081.

17. Shingate, B. B.; Hazra, B. G.; Salunke, D. B.; Pore, V. S.; Shirazi, F.; Deshpande, M. V. Eur. J. Med. Chem. 2011, 46, 3681-3689.

18. Wilson, J. E.; Casarez, A. D.; MacMillan, D. W. C. J. Am. Chem. Soc. 2009, 131, 11332-11334.

19. Zhang, M.; Liu, S.; Li, H.; Guo, Y.; Li, N.; Guan, M.; Mehfooz, H.; Zhao, J.; Zhang, Q. Chem.Eur. J. 2019, 25, 12620-12627.

20. Chen, X.-M.; Ning, X.-S.; Kang, Y.-B. Org. Lett. 2016, 18, 5368-5371.

21. Loupy, A.; Chatti, S.; Delamare, S.; Lee, D.-Y.; Chung, J.-H.; Jun, C.-H. J. Chem. Soc., Perkin Trans. 1, 2002, 1280-1285.

22. Zhao, B.; Lu, X. Org. Lett. 2006, 8, 5987-5990.

23. Li, M.; Wang, C.; Ge, H. Org. Lett. 2011, 13, 2062-2064.

24. Kose, O.; Saito, S. Org. Biomol. Chem. 2010, 8, 896-900.

25. Biju, A. T.; Glorius, F. Angew. Chem. Int. Ed. 2010, 49, 9761-9764.

26. Lewis, F. D.; Johnson, R. W.; Ruden, R. A. J. Am. Chem. Soc. 1972, 94, 4292-4297.

27. Fang, X.; Jackstell, R.; Börner, A.; Beller, M. Chem.-Eur. J. 2014, 20, 15692-15696.

28. Crawford, J. J.; Henderson, K. W.; Kerr, W. J. Org. Lett. 2006, 8, 5073-5076.

29. Wu, J.; Yang, X.; He, Z.; Mao, X.; Hatton, T. A.; Jamison, T. F. Angew. Chem. Int. Ed. 2014,

53, 8416-8420.

30. Shen, D.; Miao, C.; Xu, D.; Xia, C.; Sun, W. Org. Lett. 2015, 17, 54-57.

31. Cheng, L.-J.; Mankad, N. P. J. Am. Chem. Soc. 2017, 139, 10200-10203. 
32. Tokuyasu, T.; Kunikawa, S.; Masuyama, A.; Nojima, M. Org. Lett. 2002, 4, 3595-3598.

33. Savoia, D.; Tagliavini, E.; Trombini, C.; Umani-Ronchi, A. J. Org. Chem. 1981, 46, 5344-5348.

34. Meng, Q.-Y.; Schirmer, T. E.; Katou, K.; König, B. Angew. Chem. Int. Ed. 2019, 58, 5723-5728.

\section{NMR Spectra}




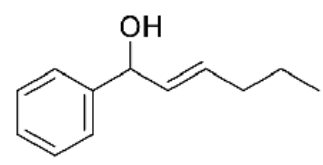

$1 c^{\prime \prime}$

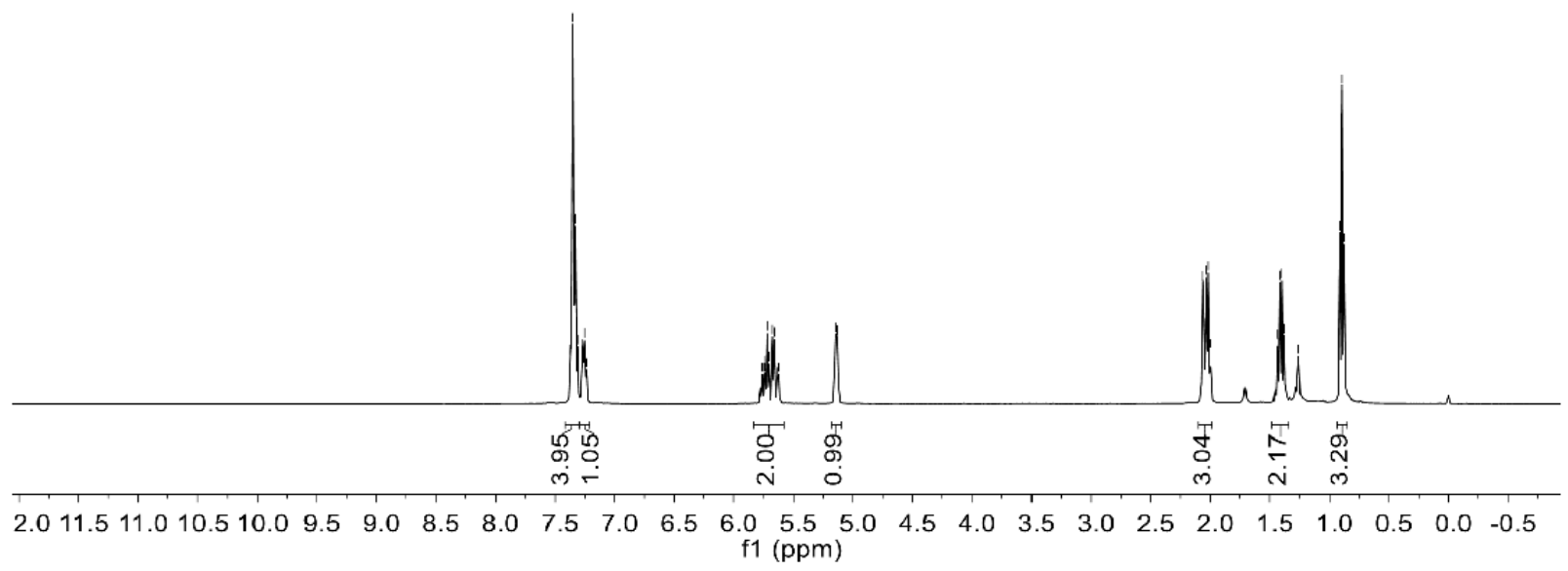

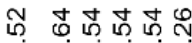

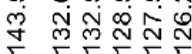

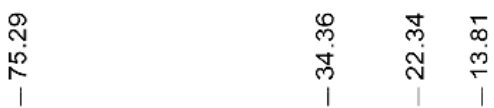

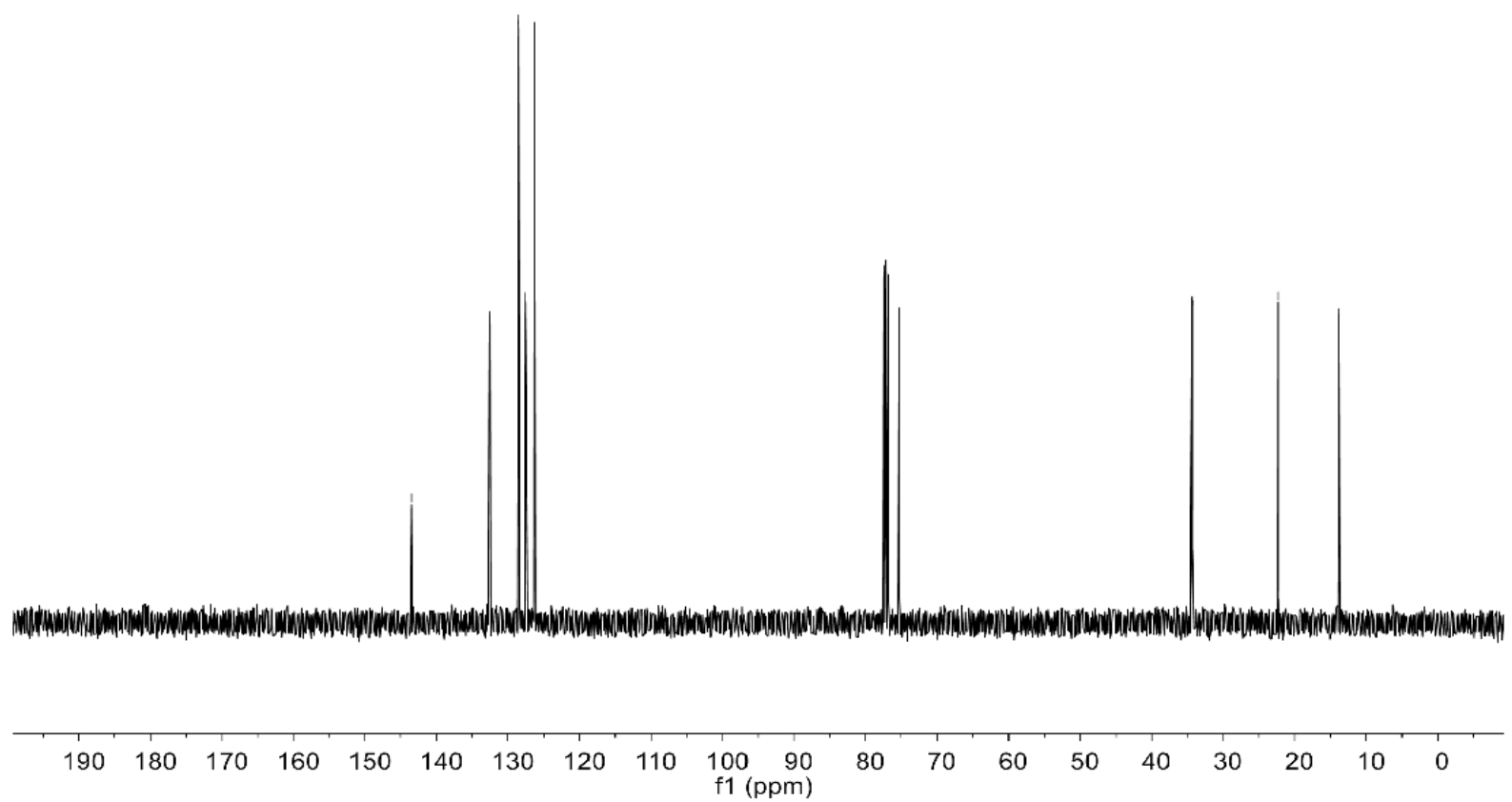




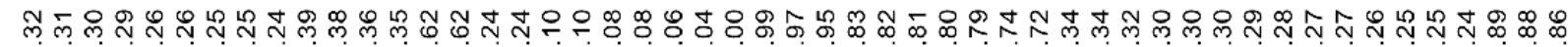

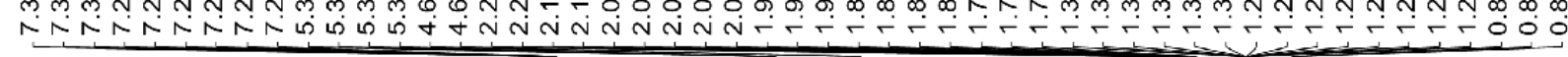
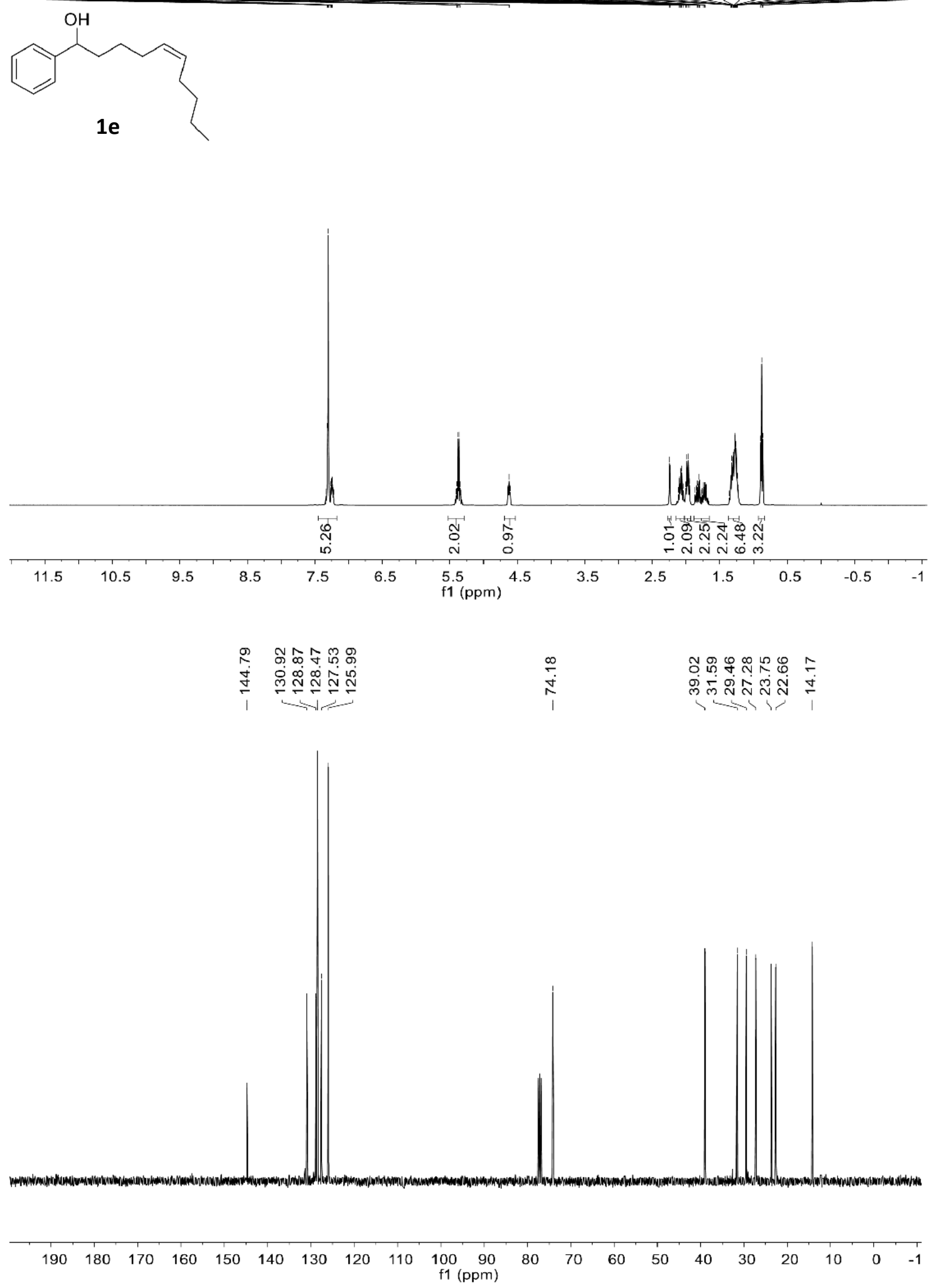
<smiles>OC(c1ccccc1)C1CC=CCC1</smiles>

$1 \mathbf{f}$
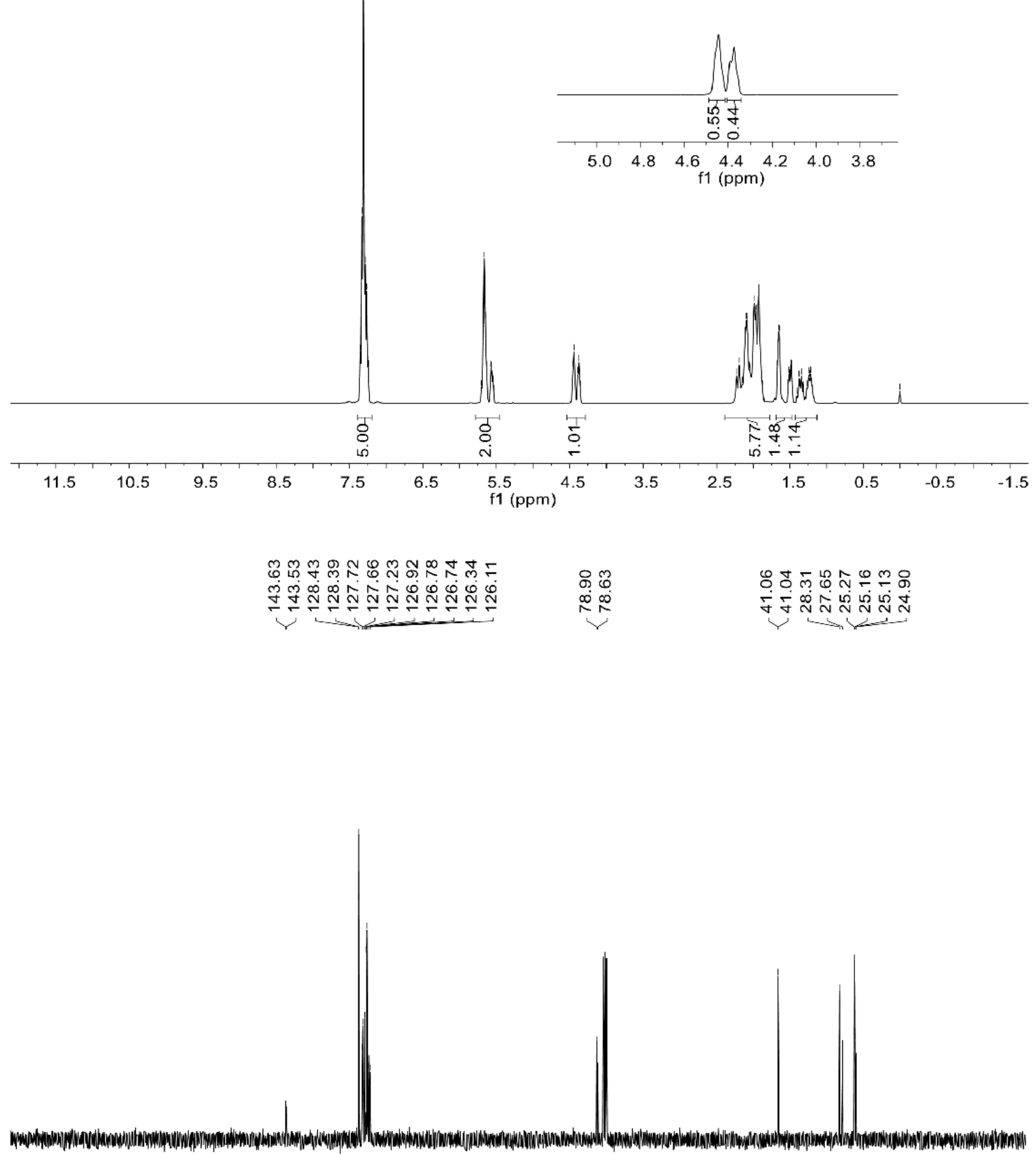


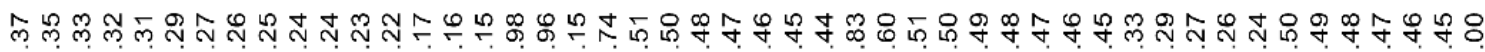

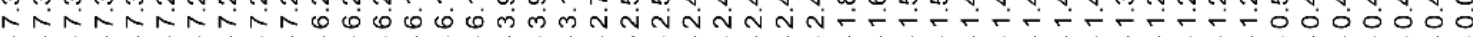<smiles>OC(c1ccccc1)C1CC2C=CC1C2</smiles>

$1 \mathrm{~g}$

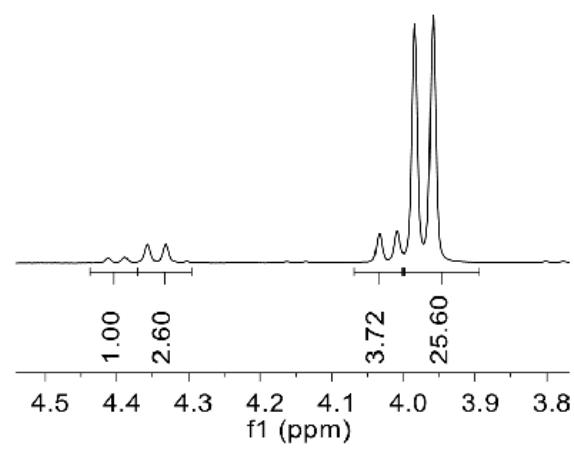

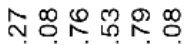

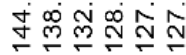
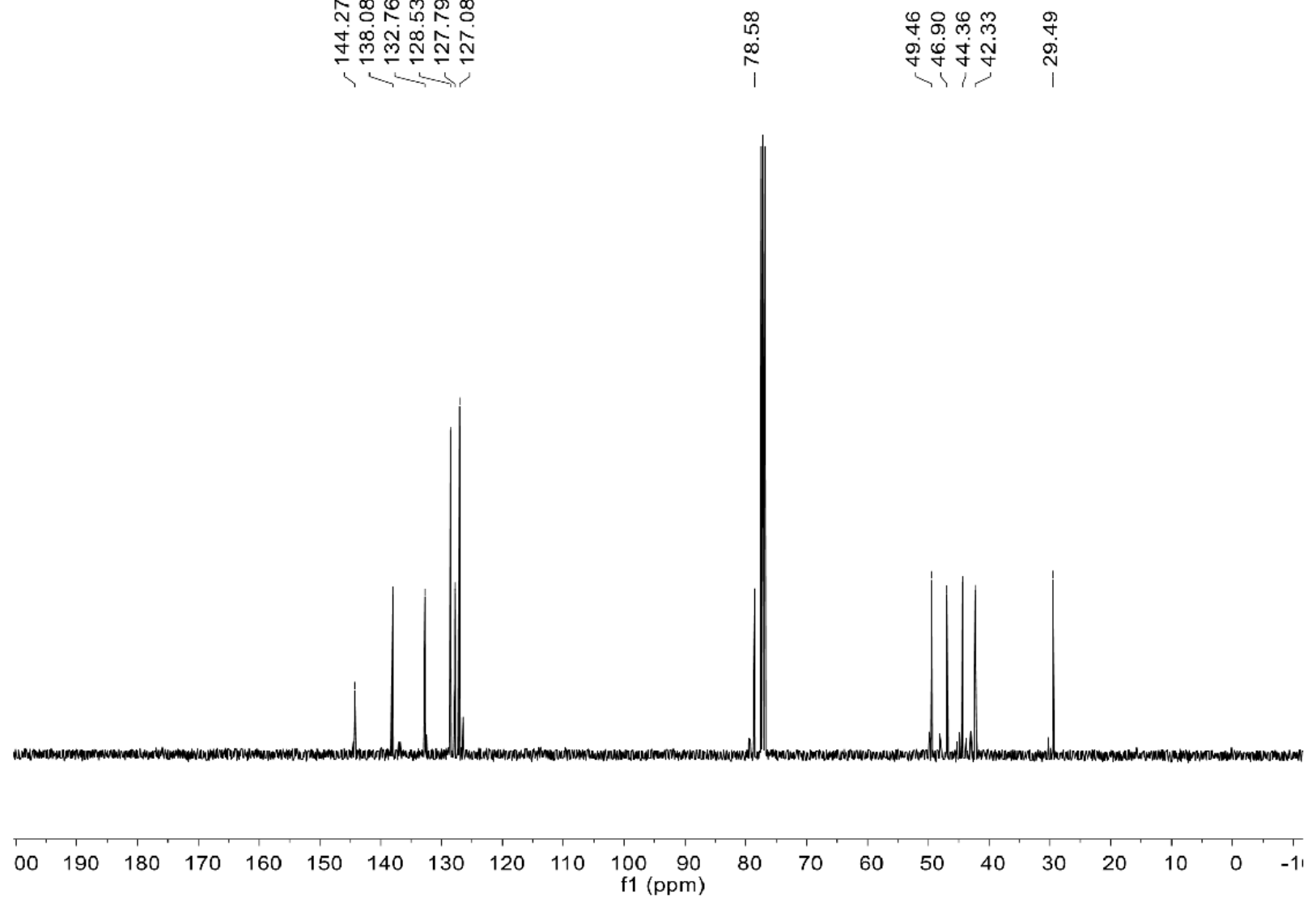


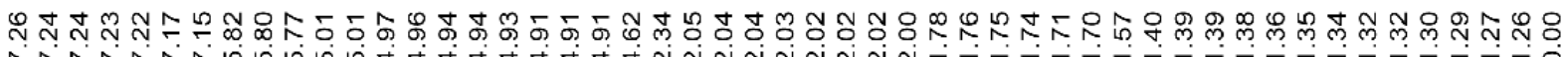

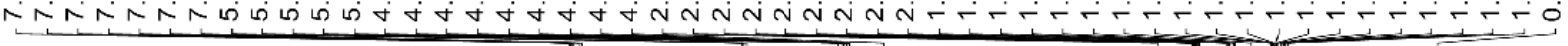

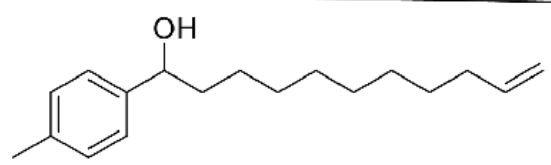

$1 \mathrm{~h}$

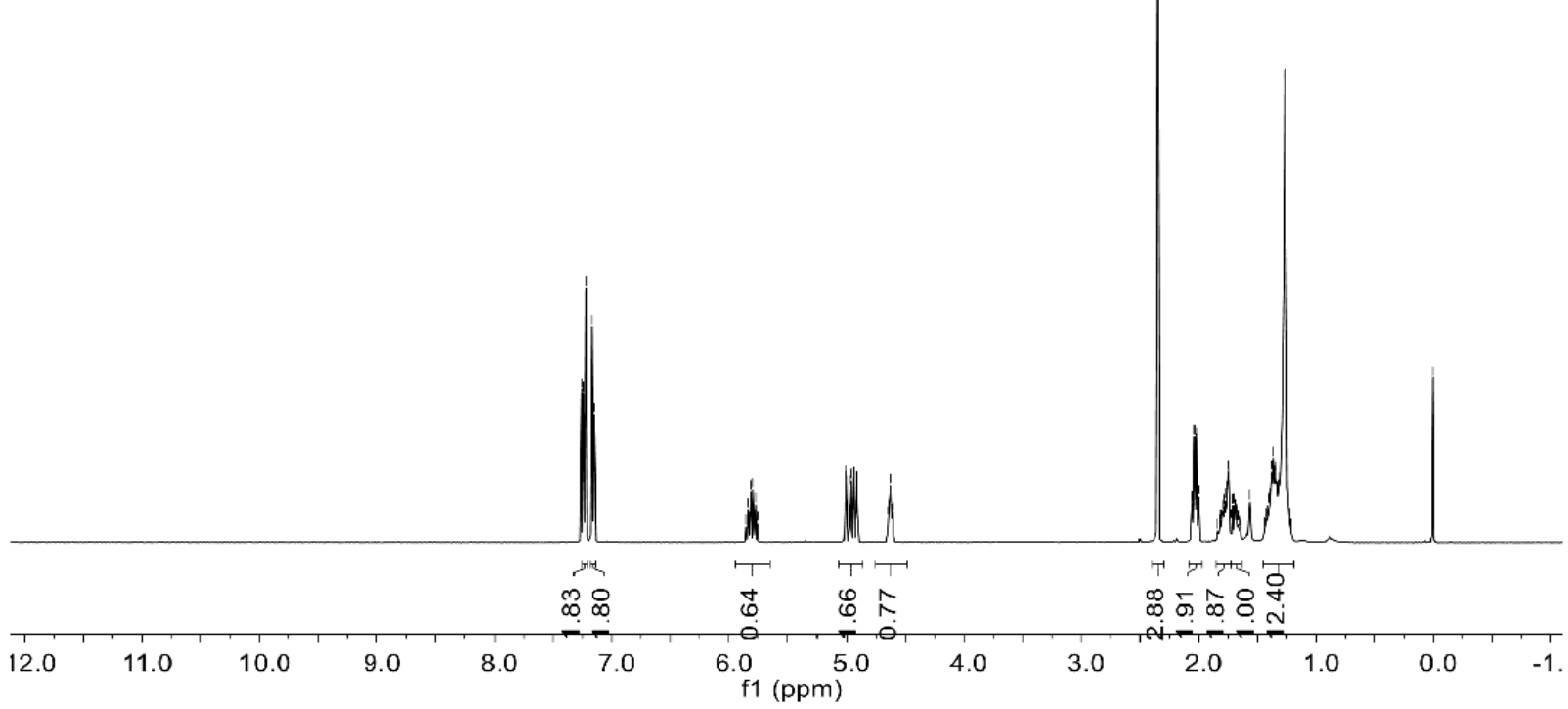

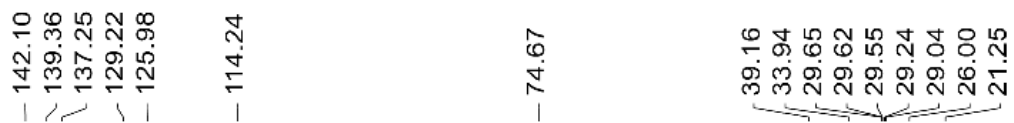

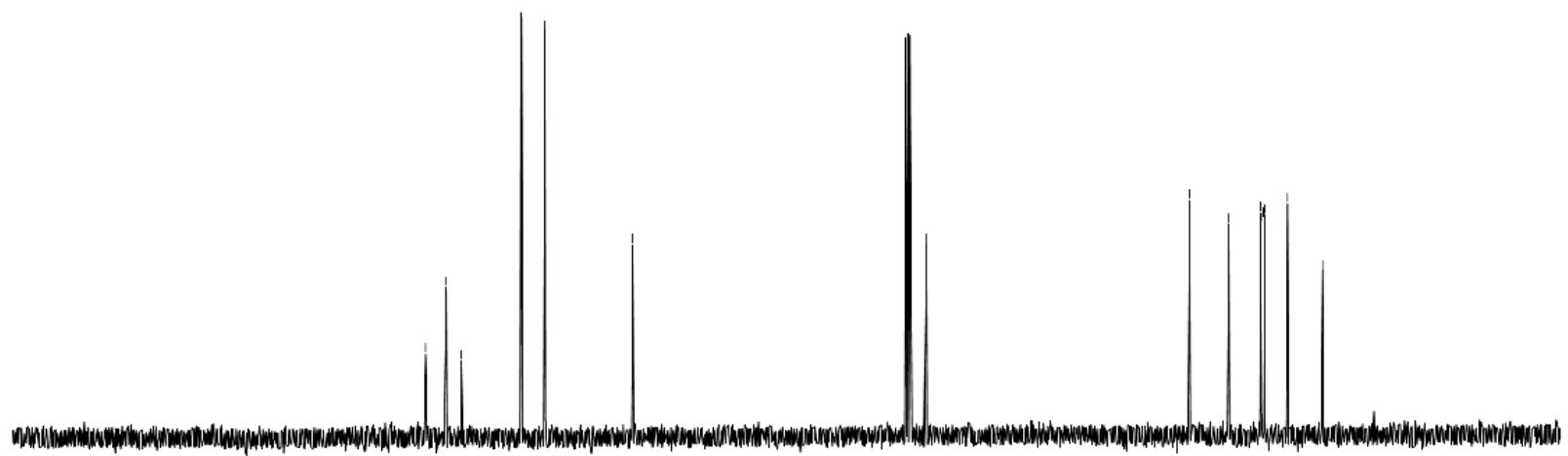

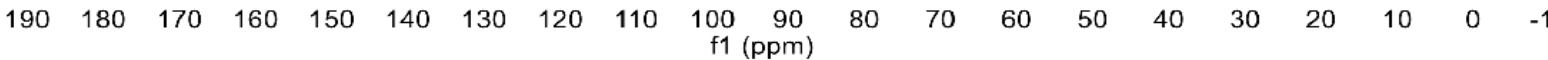



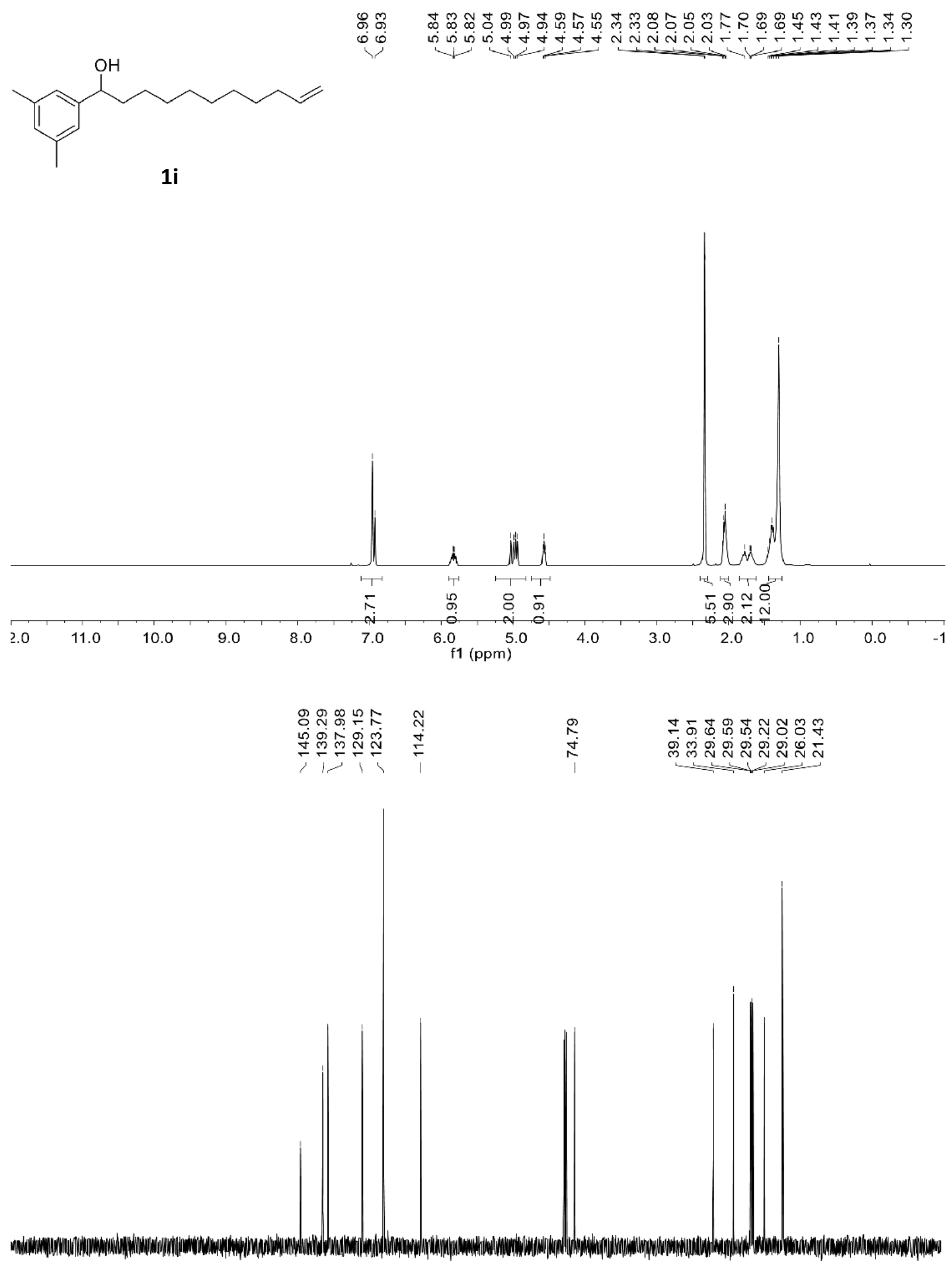

$\begin{array}{llllllllllllllllllllll}210 & 200 & 190 & 180 & 170 & 160 & 150 & 140 & 130 & 120 & \begin{array}{c}110 \\ \mathrm{f} 1\end{array} \begin{array}{l}100 \\ (\mathrm{ppm})\end{array} & 90 & 80 & 70 & 60 & 50 & 40 & 30 & 20 & 10 & 0 & -10\end{array}$ 


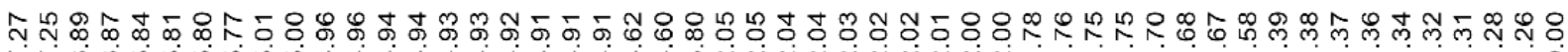

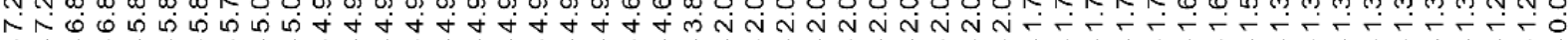<smiles>C=CCCCCCCCCC(O)c1ccc(OC)cc1</smiles>

$1 \mathbf{j}$

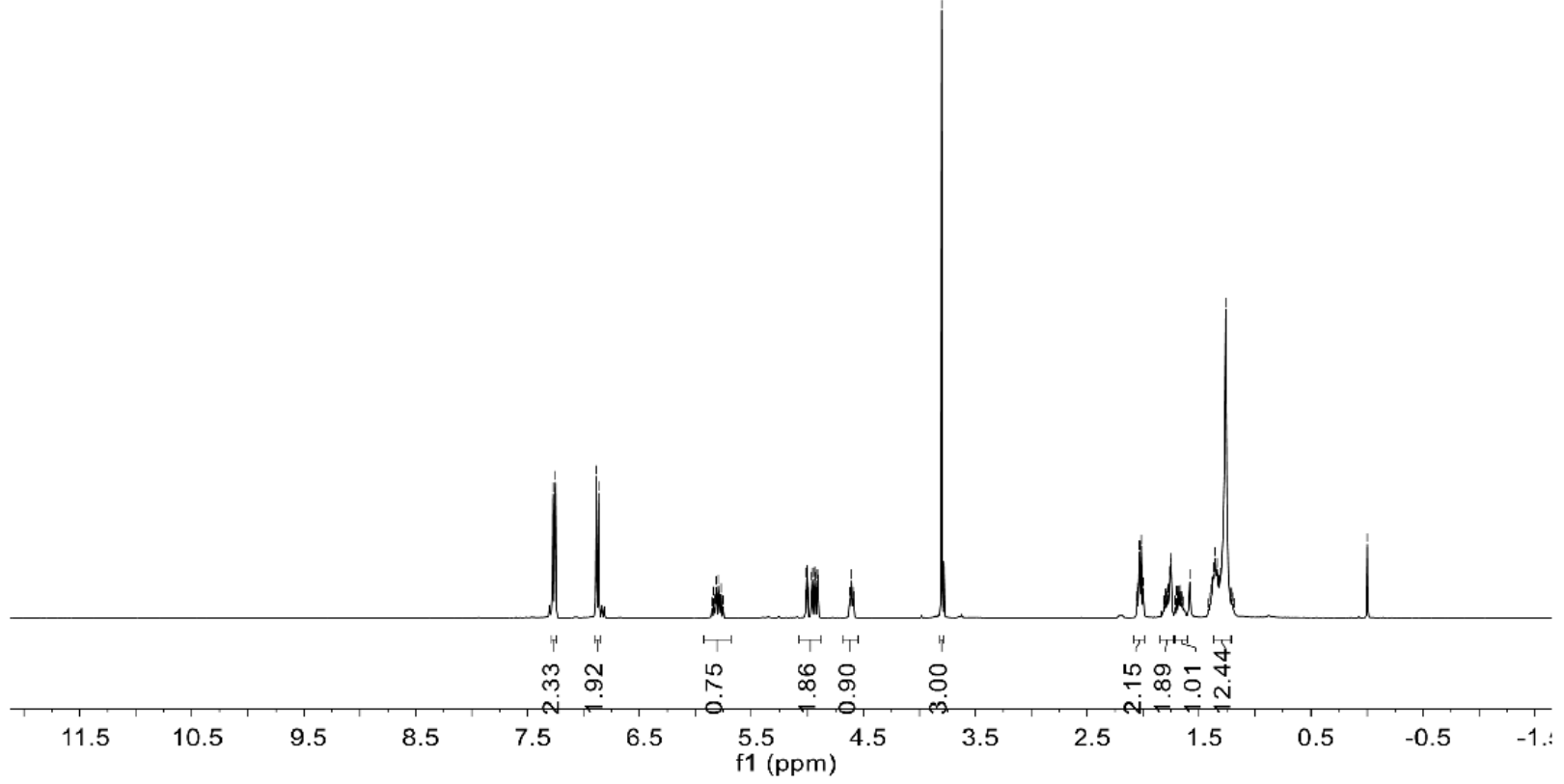

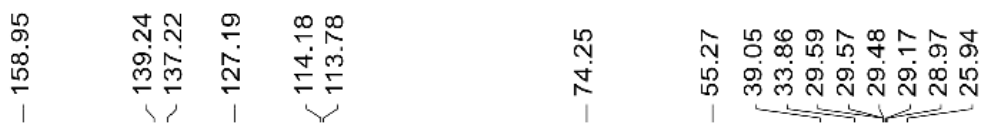

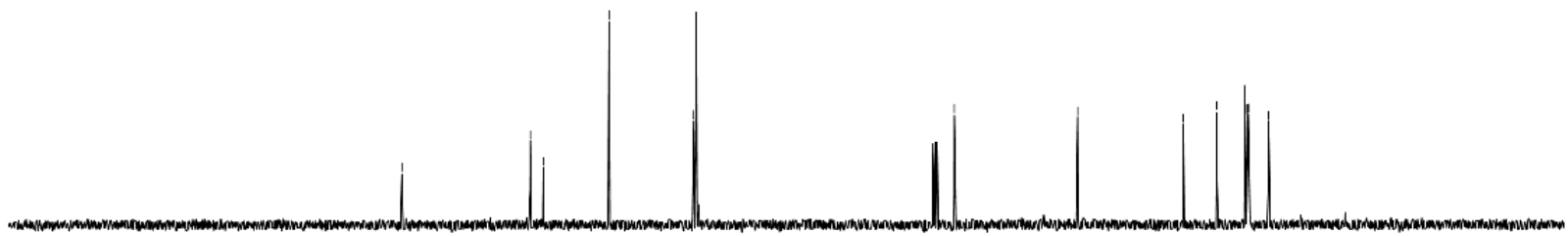

$\begin{array}{lllllllllllllllllllllll}210 & 200 & 190 & 180 & 170 & 160 & 150 & 140 & 130 & 120 & \begin{array}{c}110 \\ \mathrm{f} 1\end{array} \begin{array}{l}100 \\ (\mathrm{ppm})\end{array} & 90 & 80 & 70 & 60 & 50 & 40 & 30 & 20 & 10 & 0 & -10\end{array}$ 


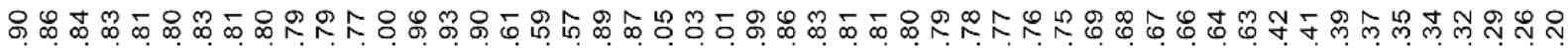

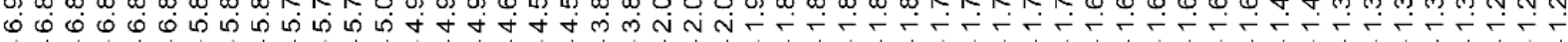<smiles>C=CCCCCCCCCC(O)c1ccc(OC)c(OC)c1</smiles>

$1 \mathbf{k}$
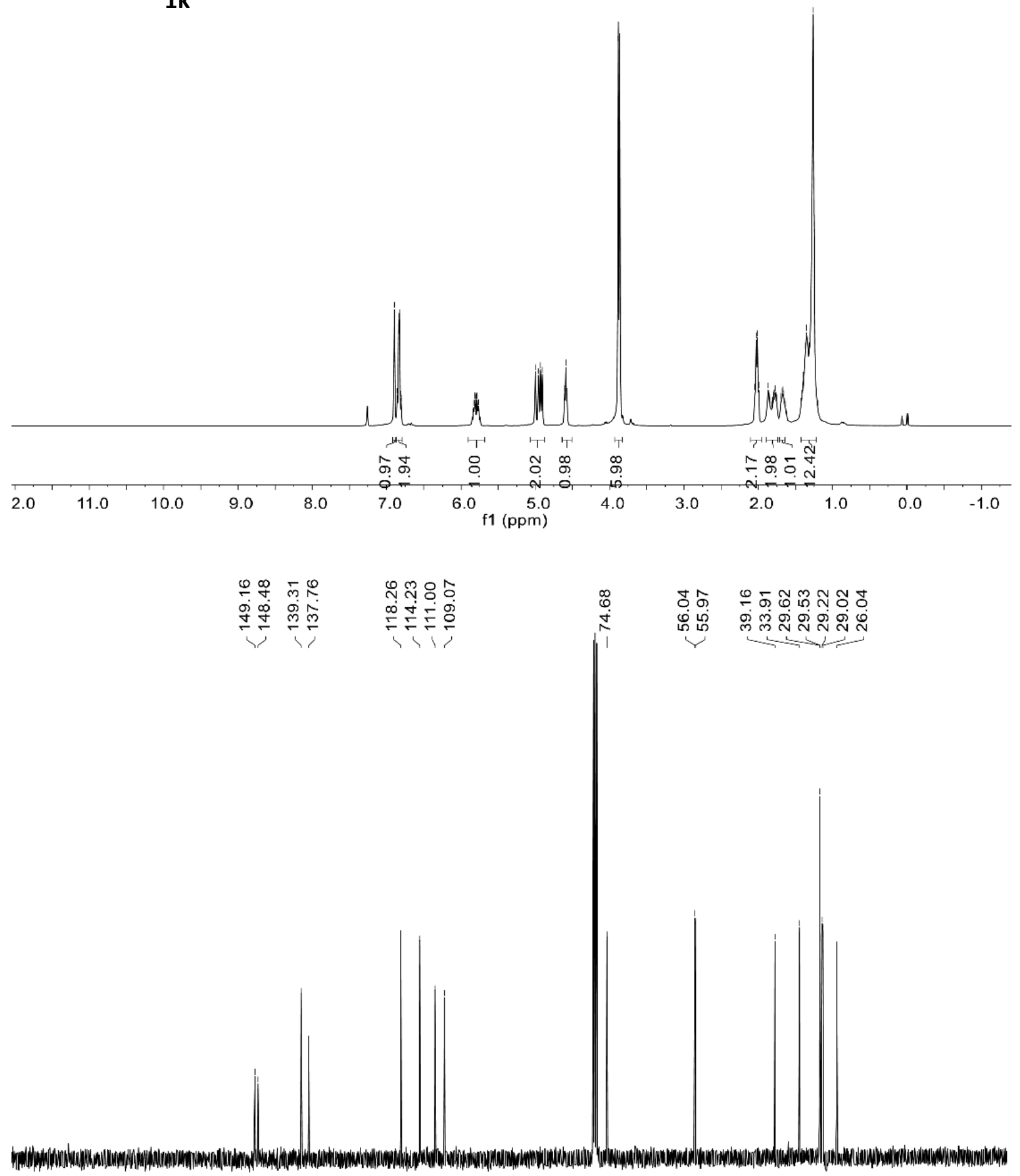

$\begin{array}{llllllllllllllllllllll}00 & 190 & 180 & 170 & 160 & 150 & 140 & 130 & 120 & 110 & \begin{array}{c}100 \\ \mathrm{f} 1(\mathrm{ppm})\end{array} & 90 & 70 & 60 & 50 & 40 & 30 & 20 & 10 & 0 & -\end{array}$ 


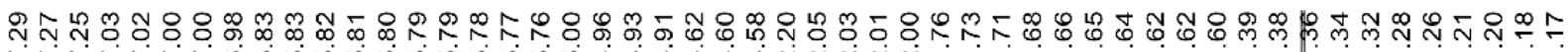

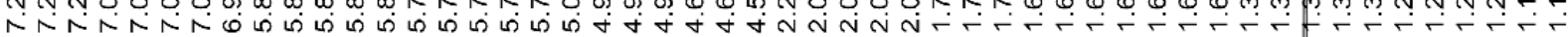

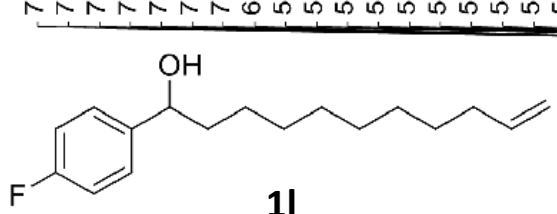

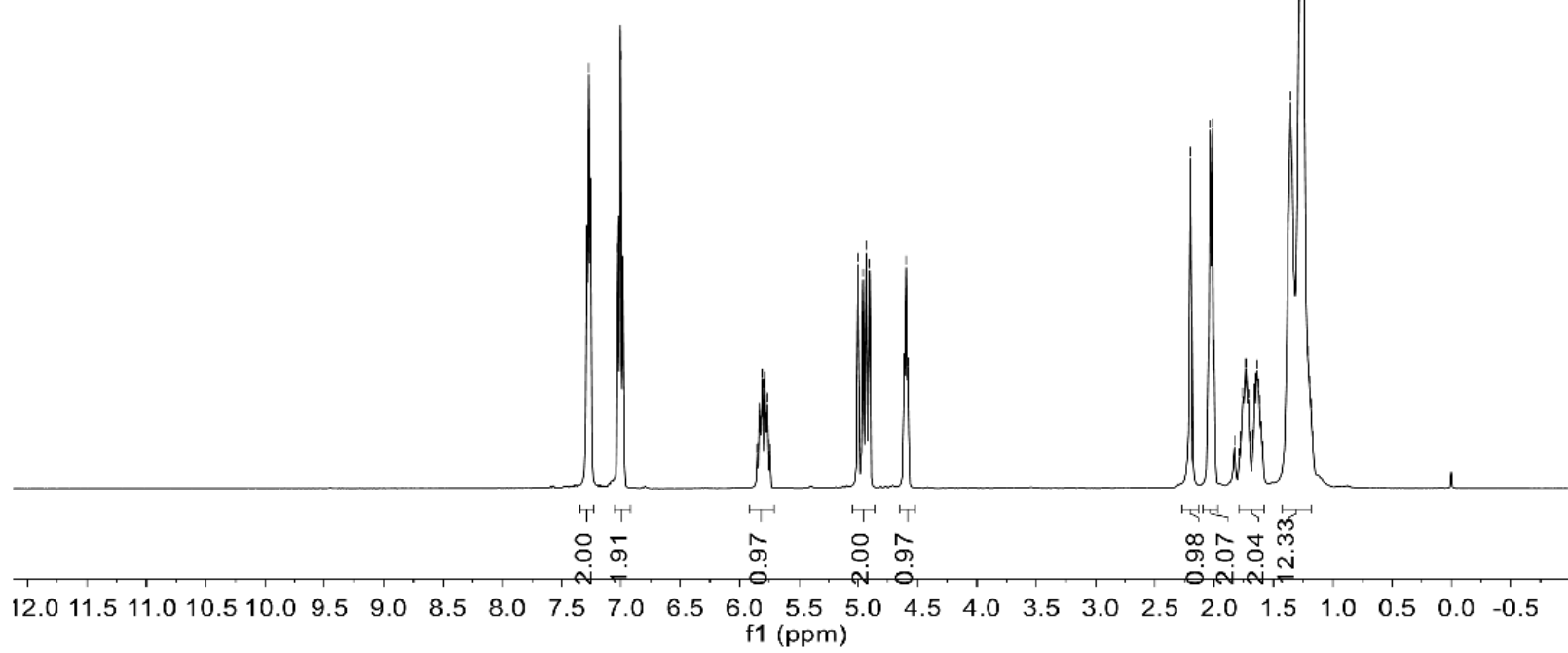

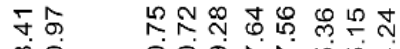

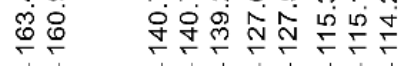

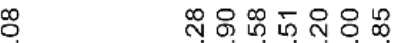

i

$\underbrace{-i j}_{i}$

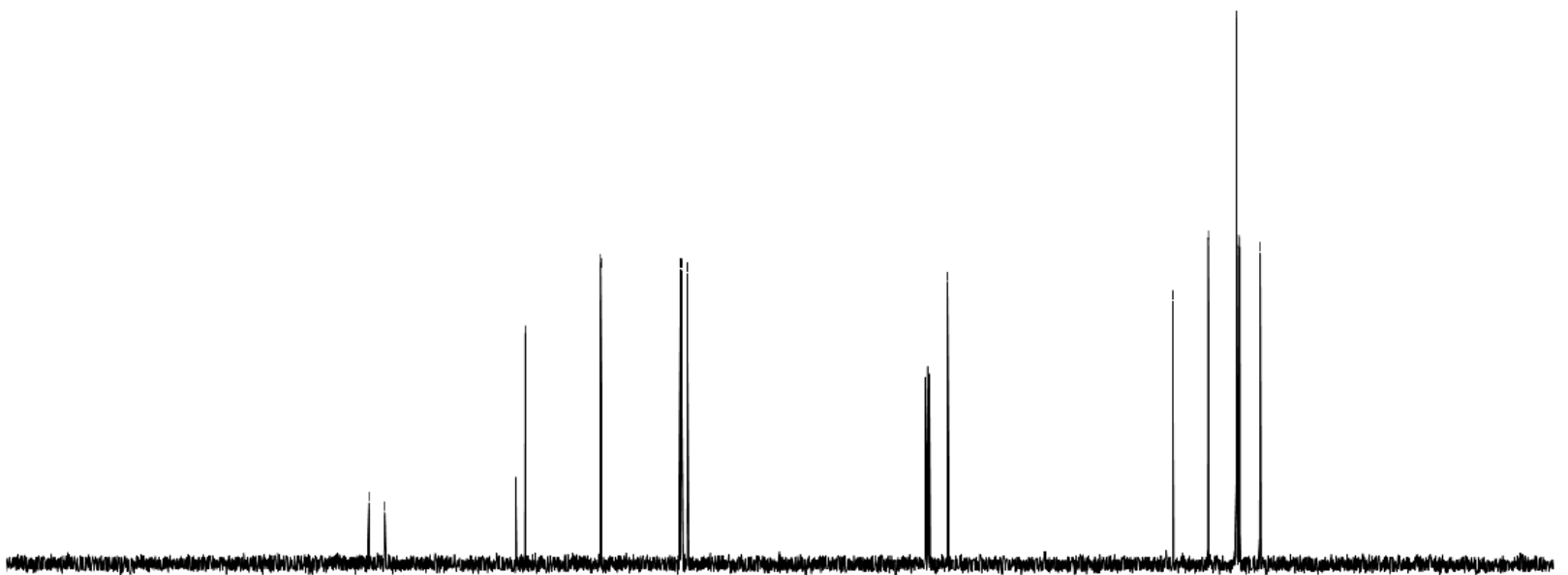

$\begin{array}{lllllllllllllllllllllll}210 & 200 & 190 & 180 & 170 & 160 & 150 & 140 & 130 & 120 & 110 & 100 & 90 & 80 & 70 & 60 & 50 & 40 & 30 & 20 & 10 & 0 & -10\end{array}$ 


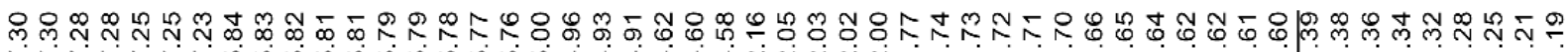

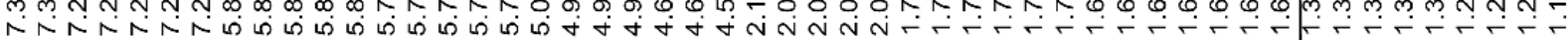<smiles>C=CCCCCCCCCC(O)c1ccc(Cl)cc1</smiles>

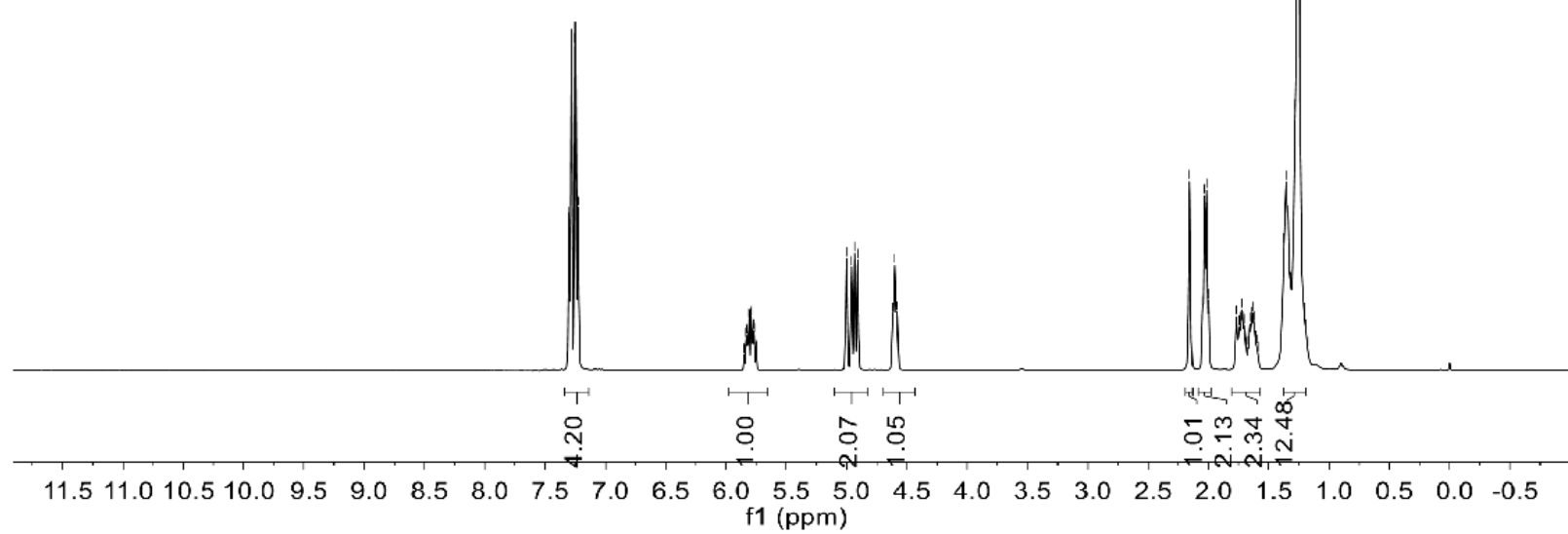

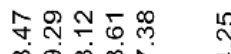

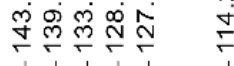

i
$\stackrel{1}{0}$

굥ำ

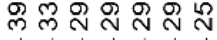

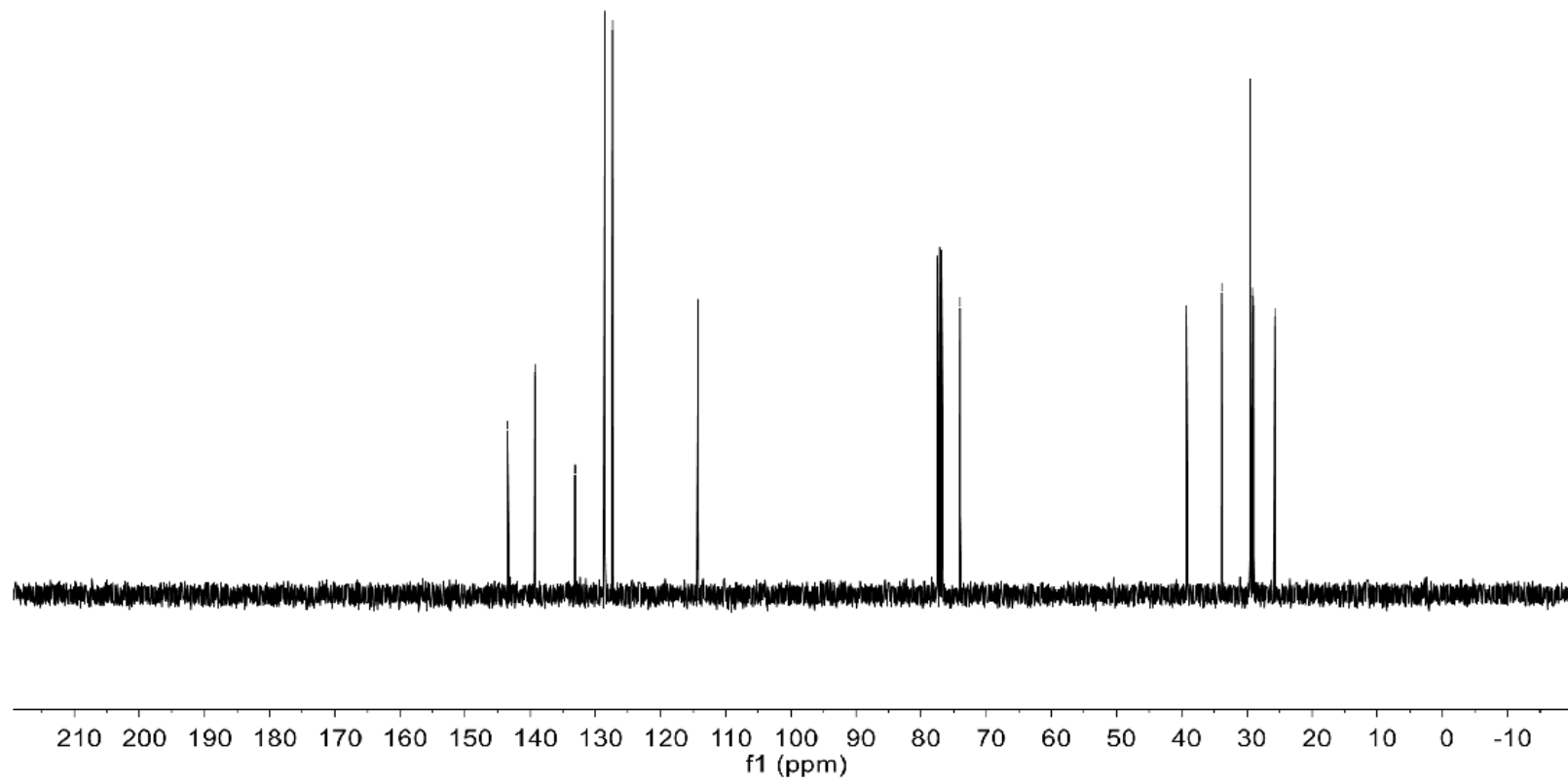




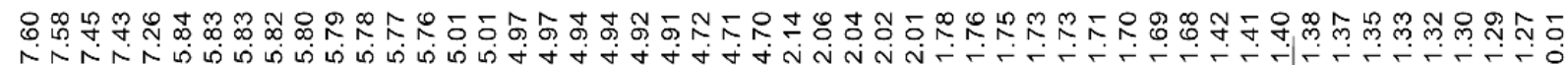

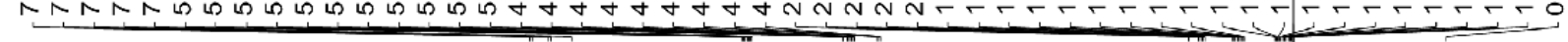<smiles>C=CCCCCCCCCC(O)c1ccc(C(F)(F)F)cc1</smiles>

1n

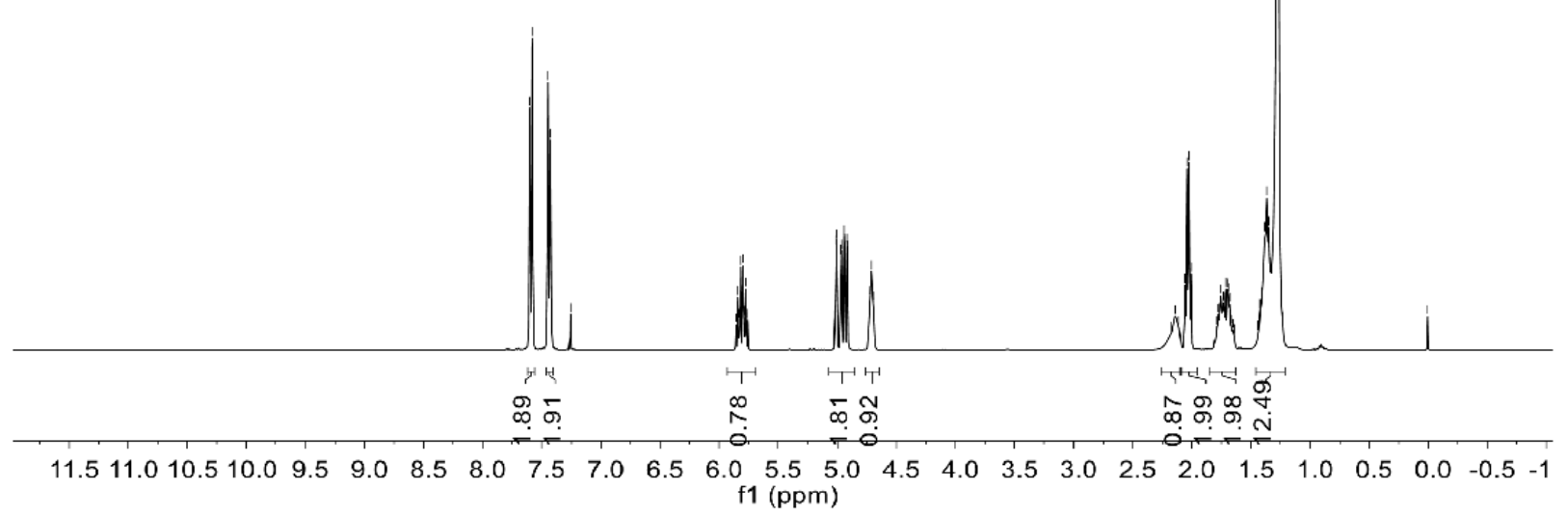

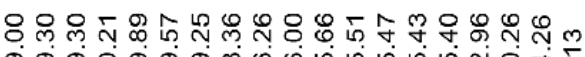

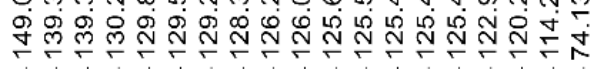

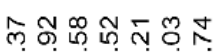
ल्లल

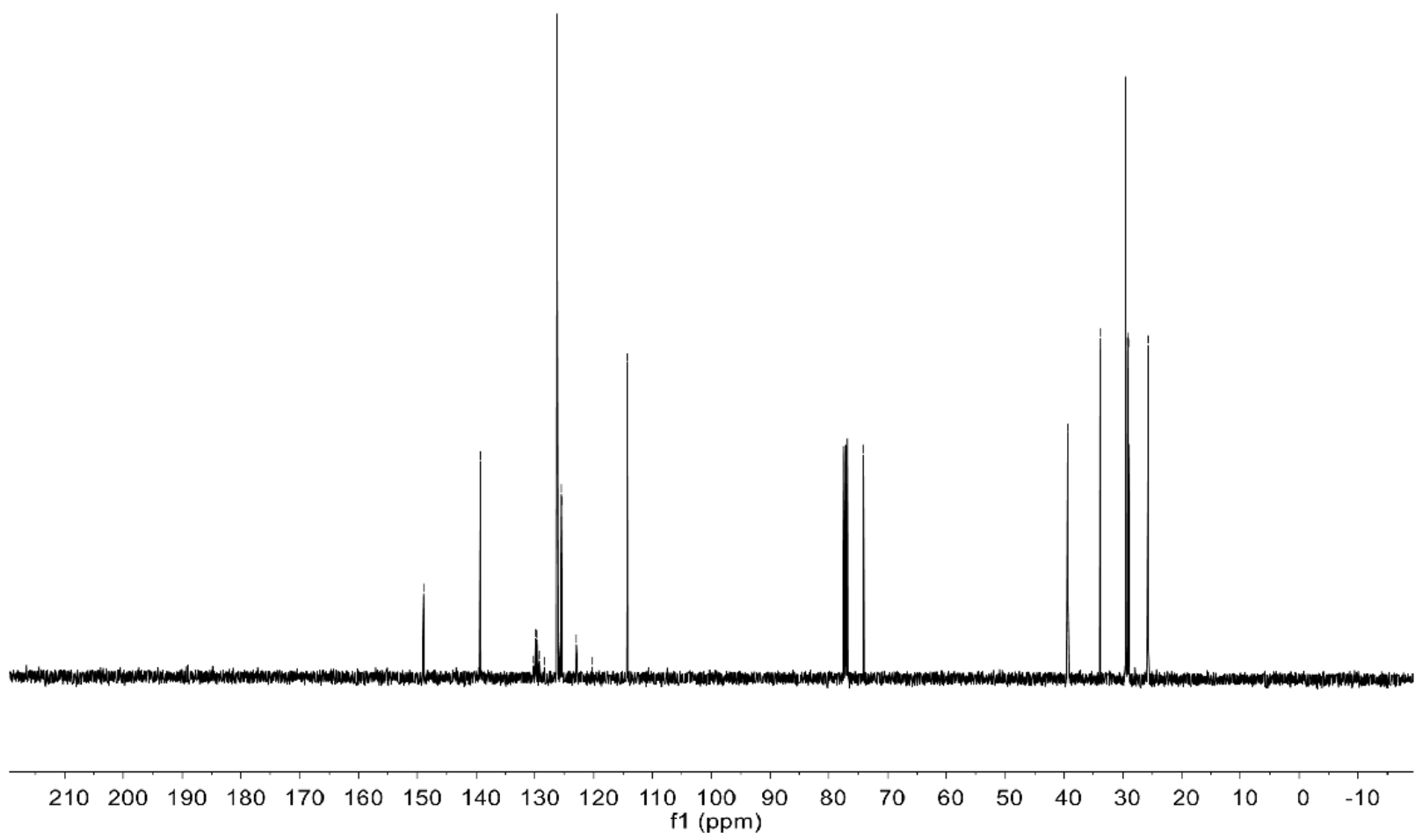




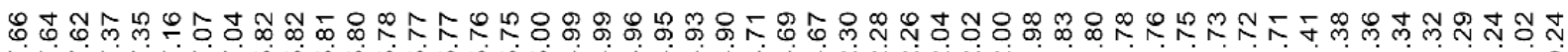

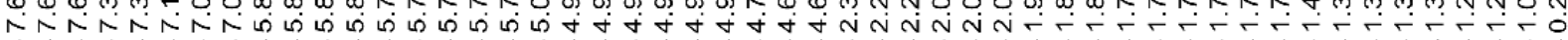<smiles>C=CCCCCCCCCC(O)c1ccc2cc(OCCC)ccc2c1</smiles>

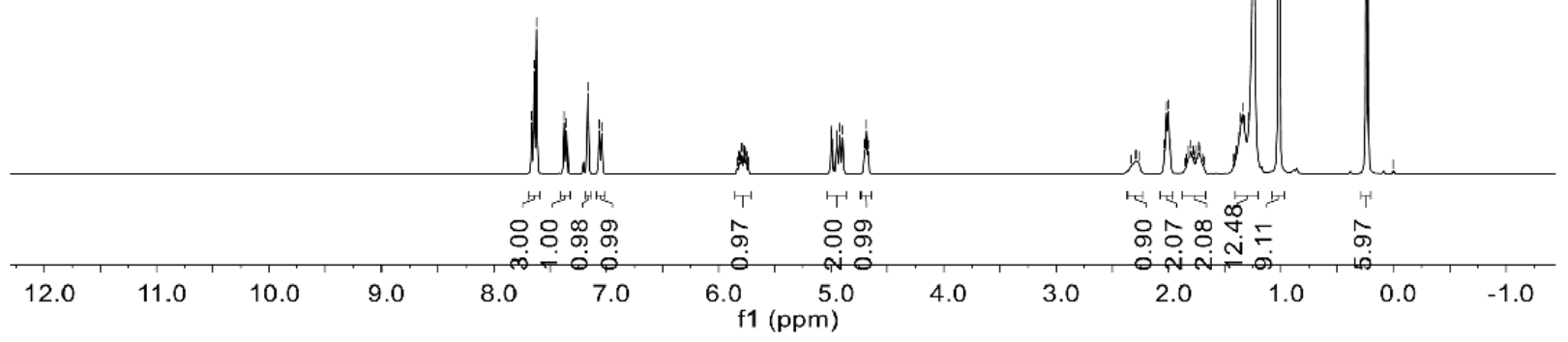

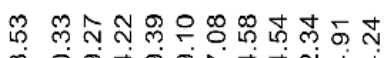

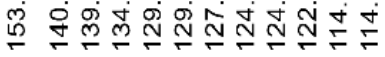

बढ̆

品

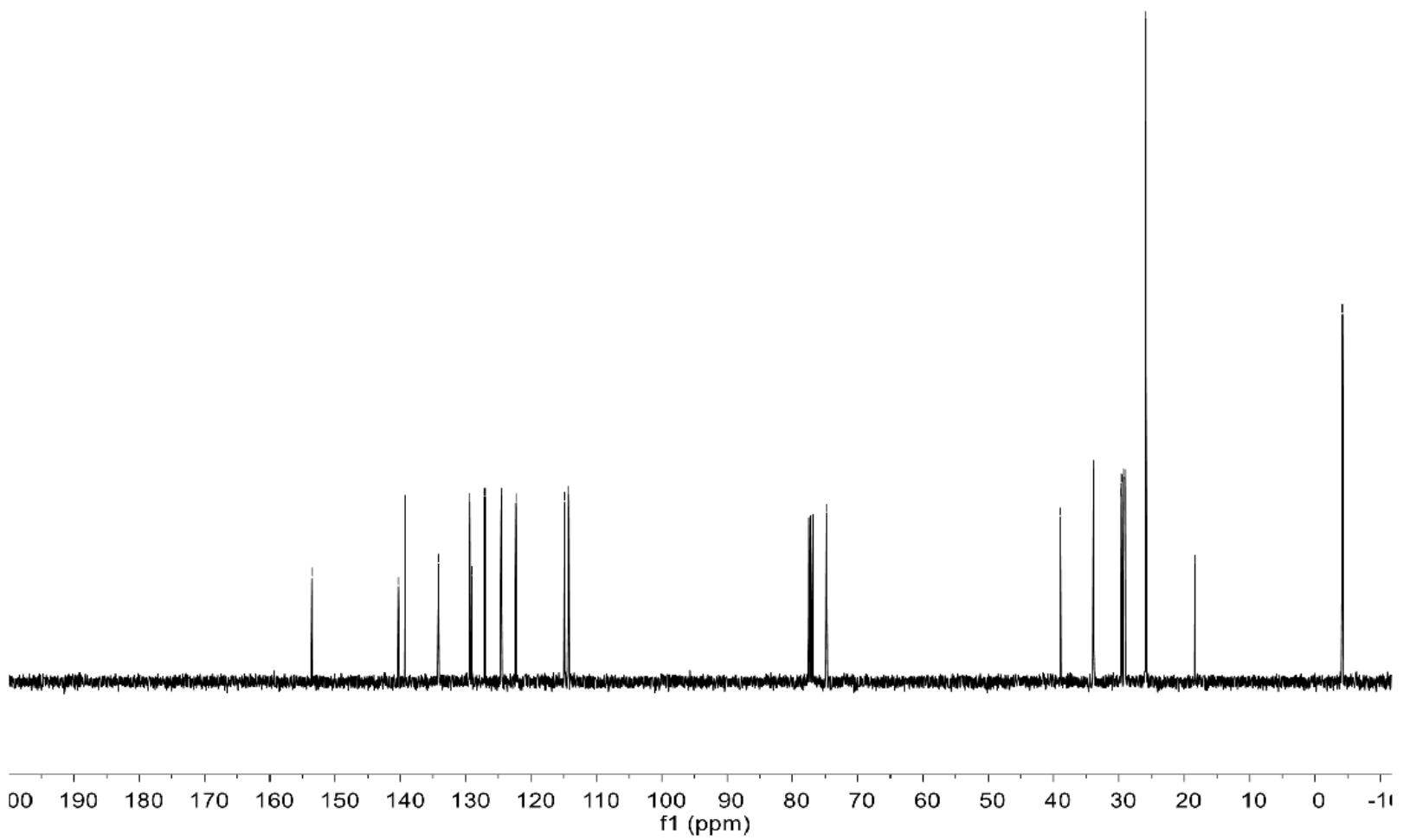



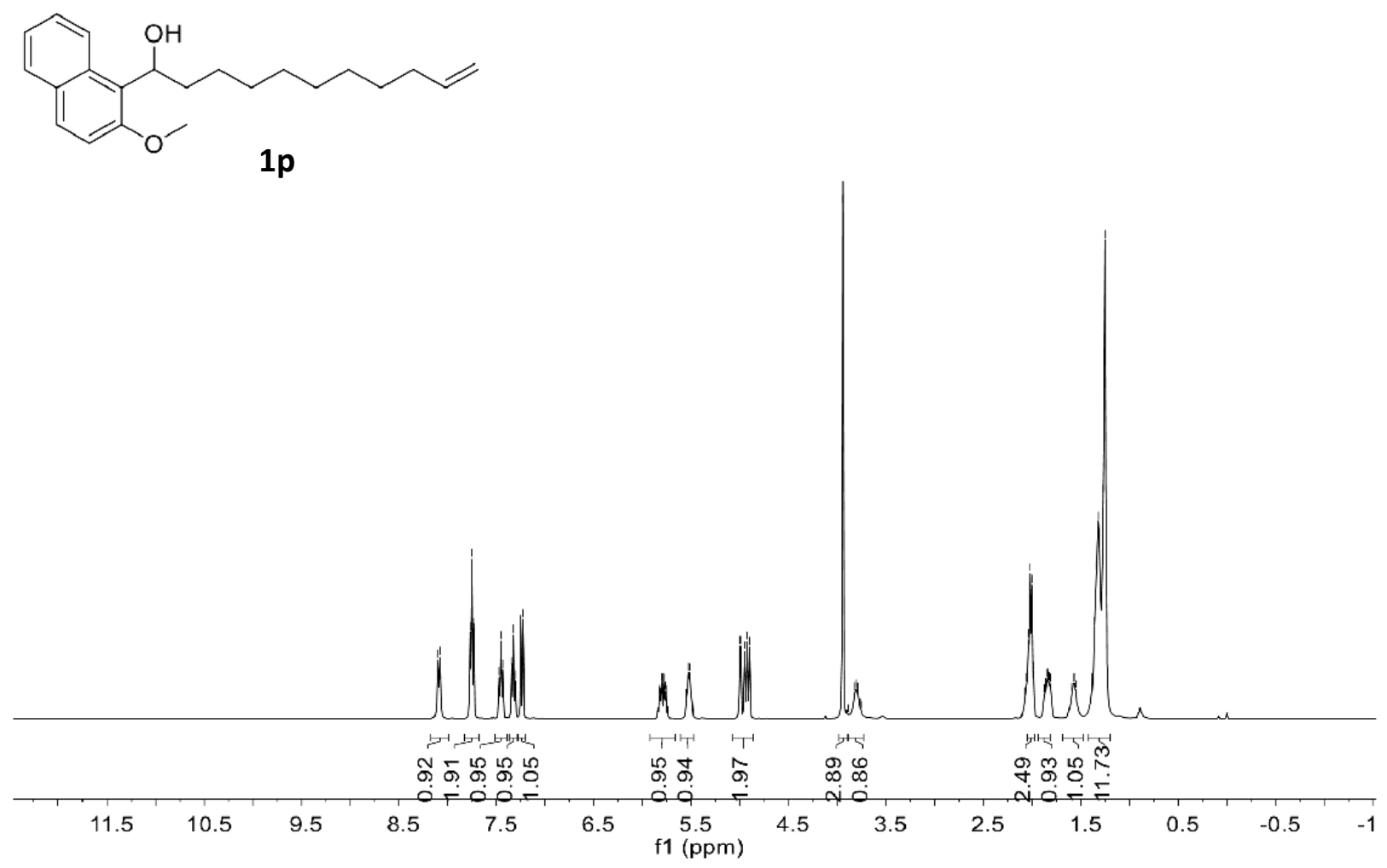

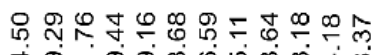

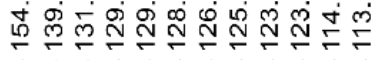

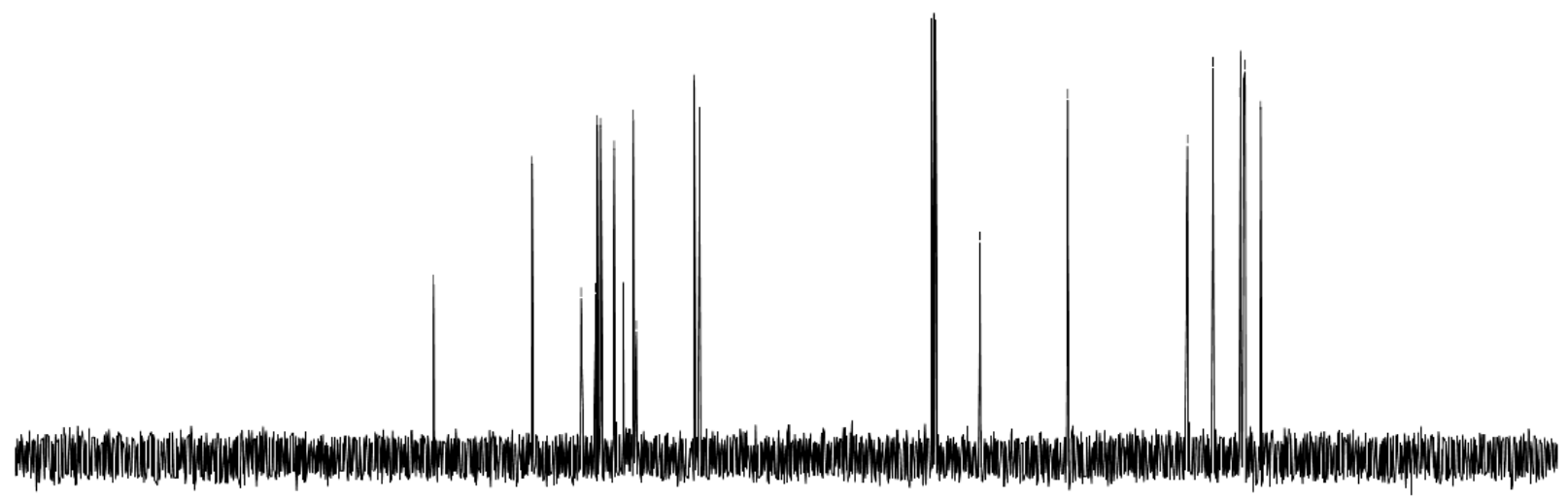

$\begin{array}{lllllllllllll}210 & 200 & 190 & 180 & 170 & 160 & 150 & 140 & 130 & 120 & 110 & 100 & 90\end{array}$ 


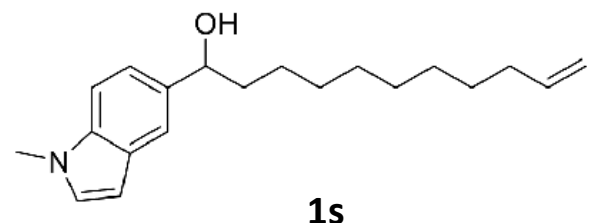

1s

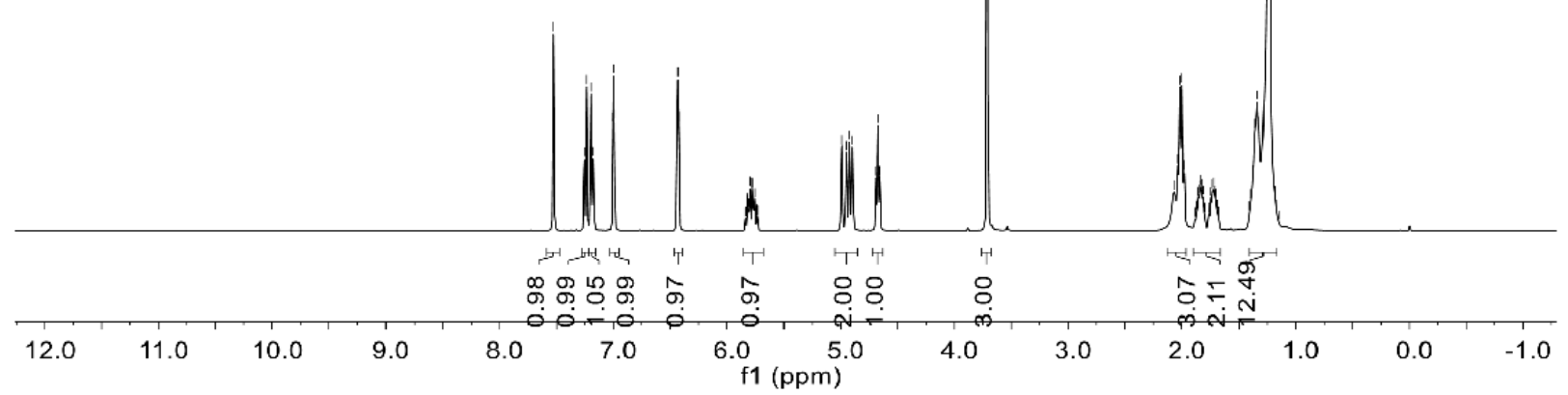

กิ m

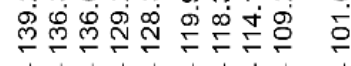

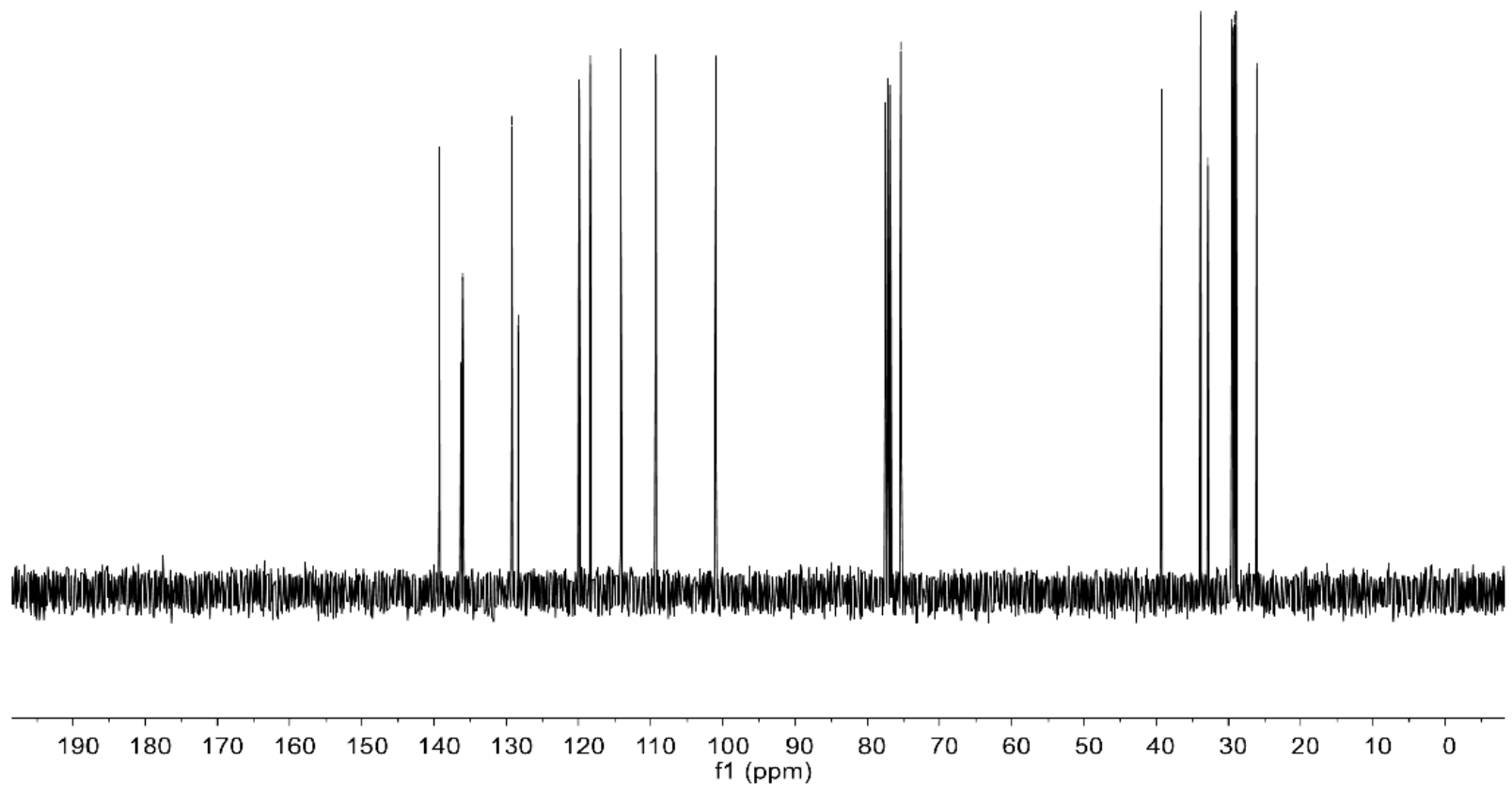


幽品 rín

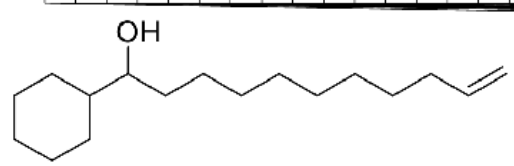

1u

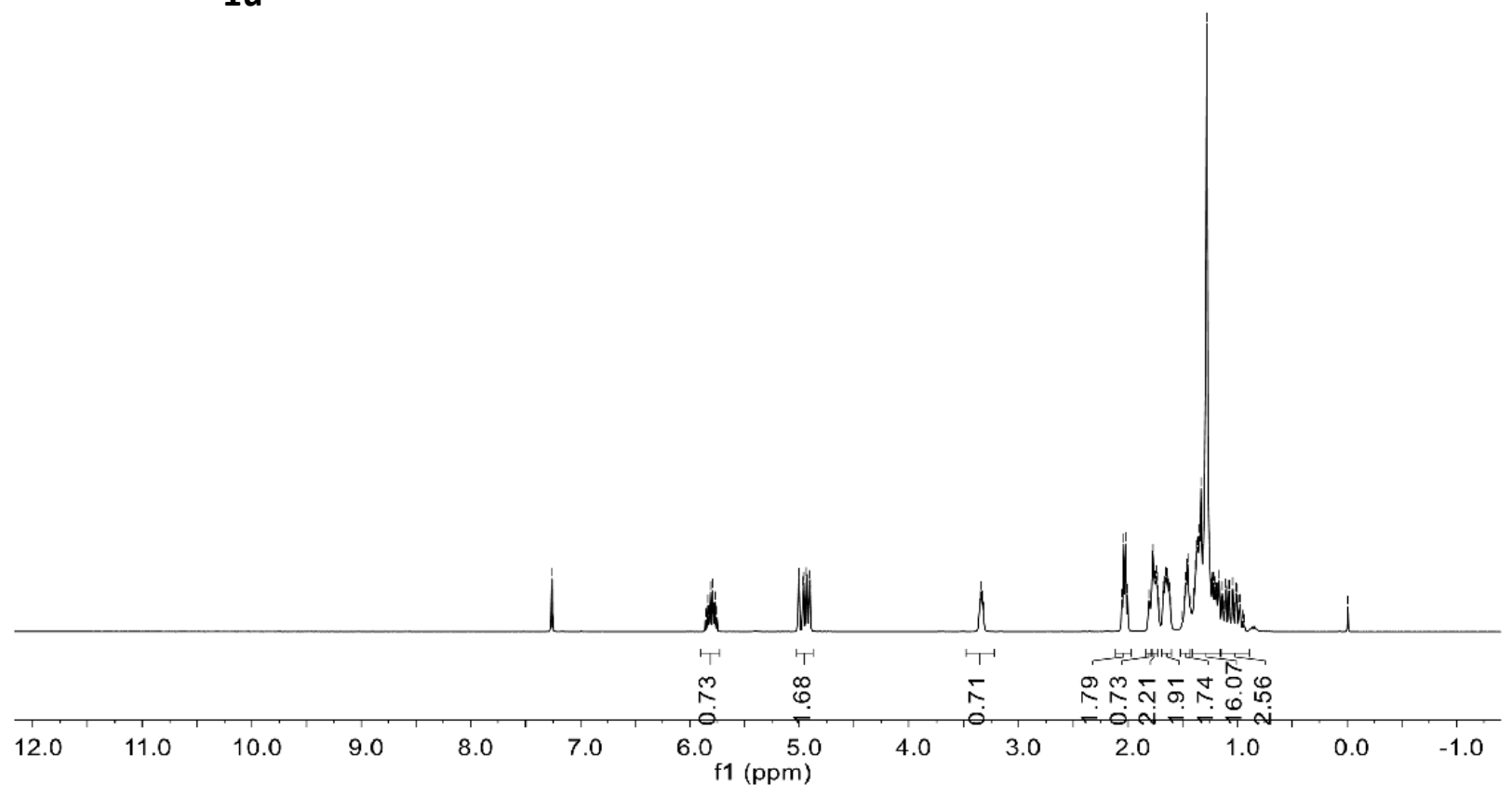

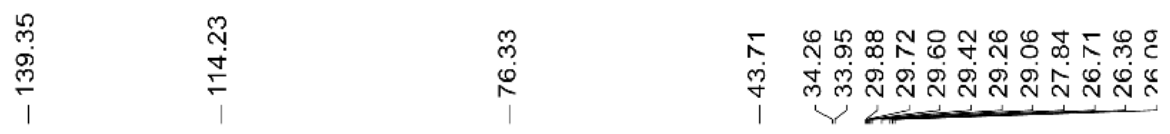

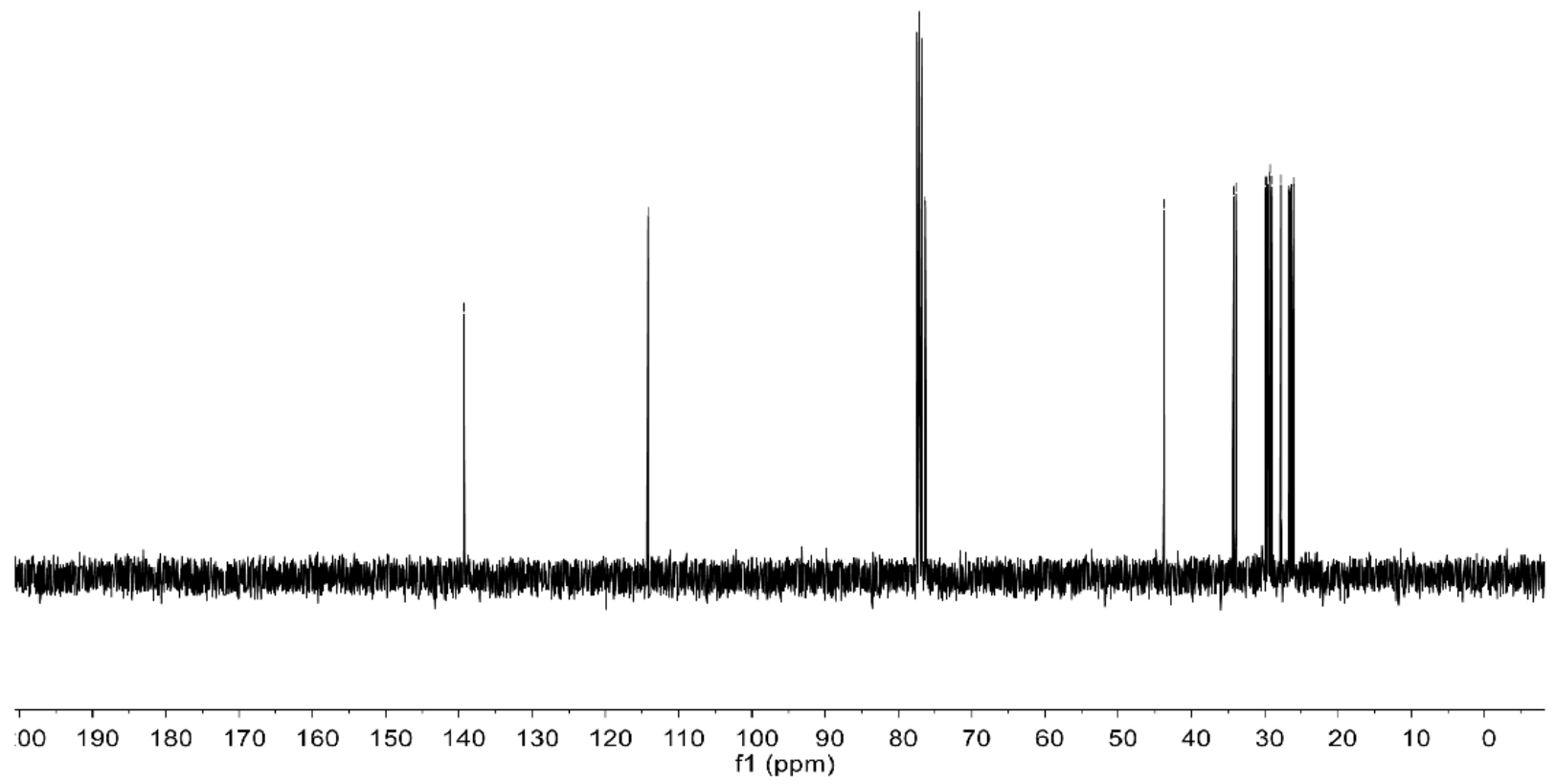




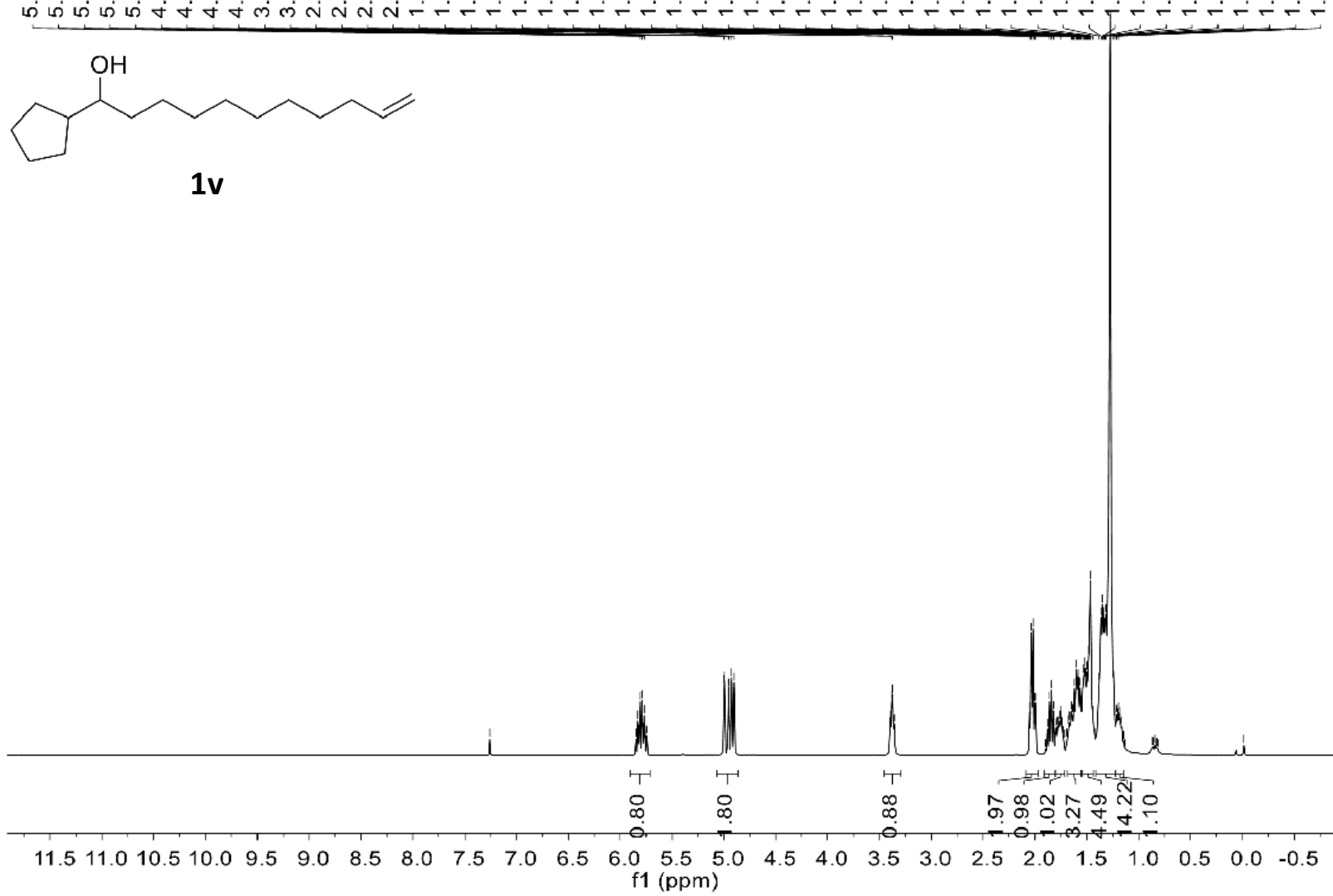

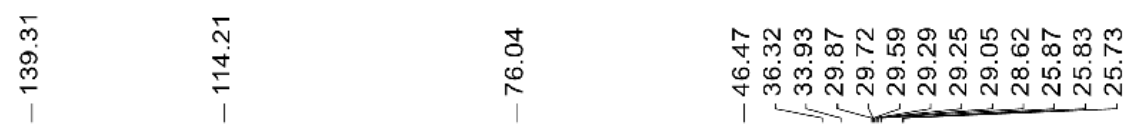

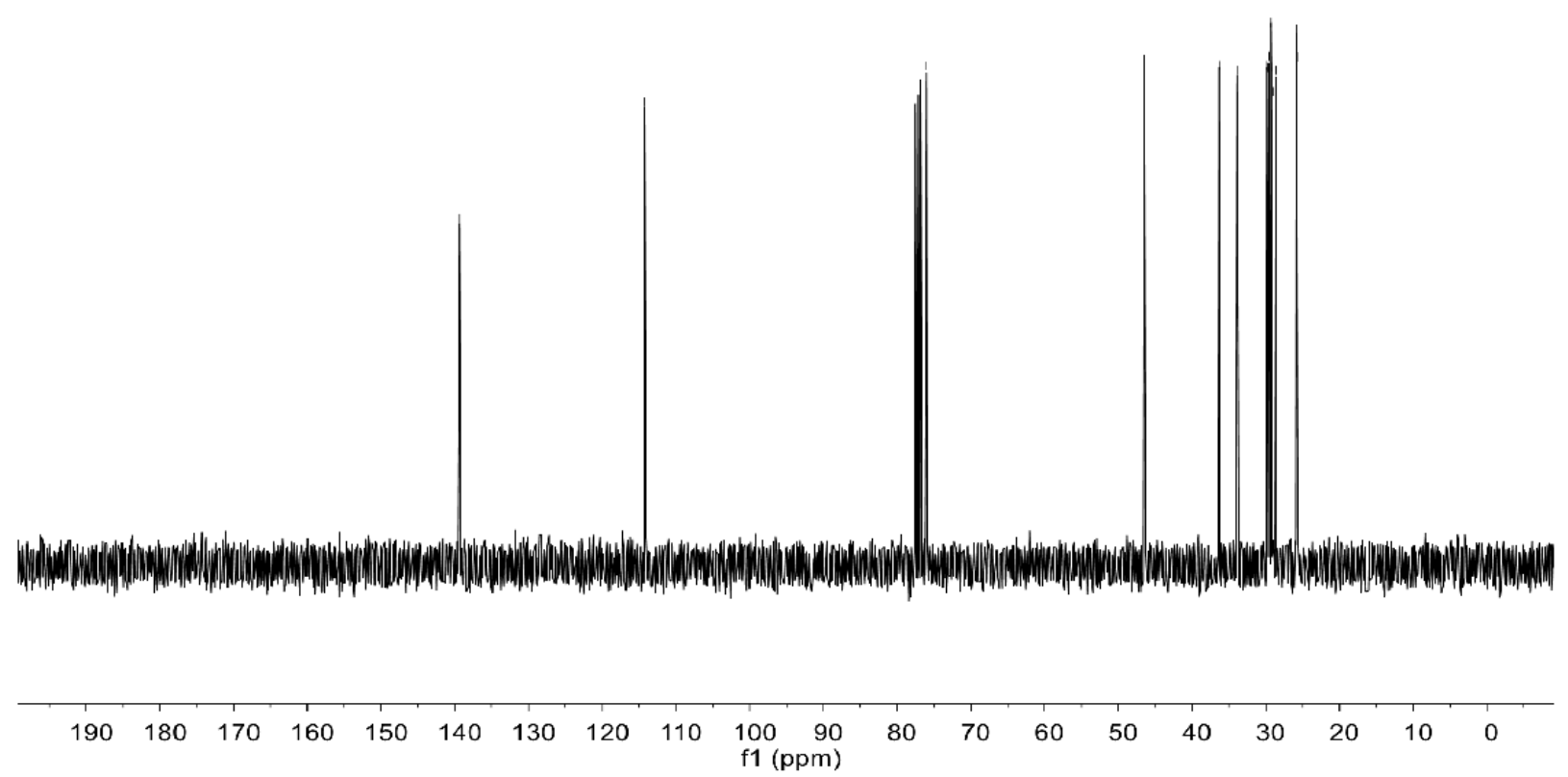




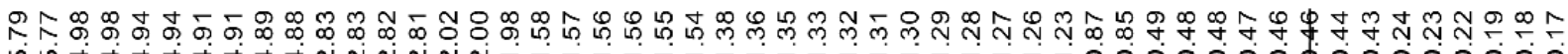

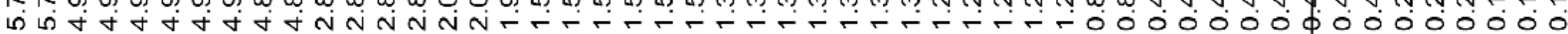

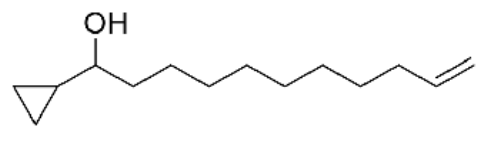

$1 w$

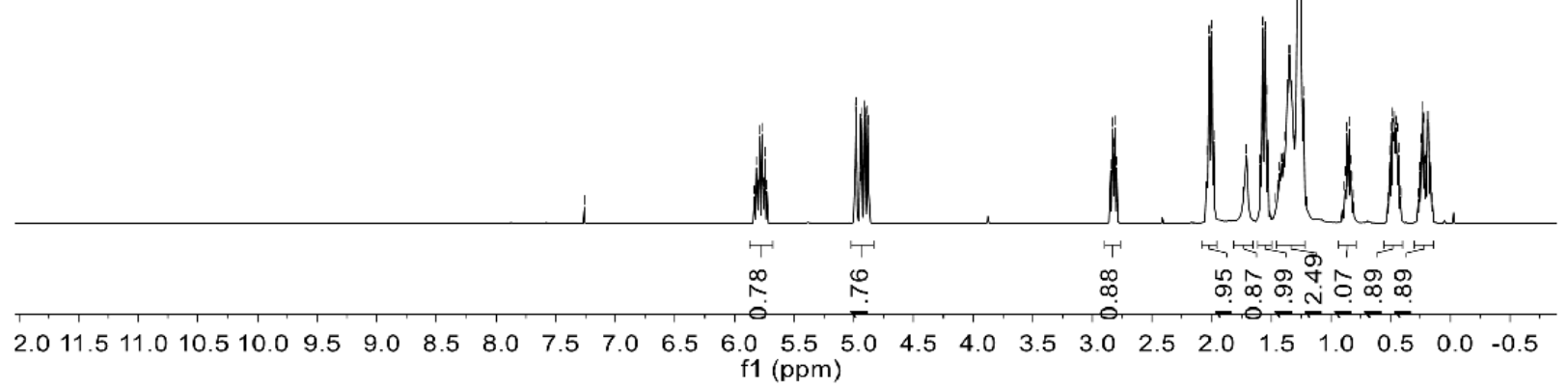

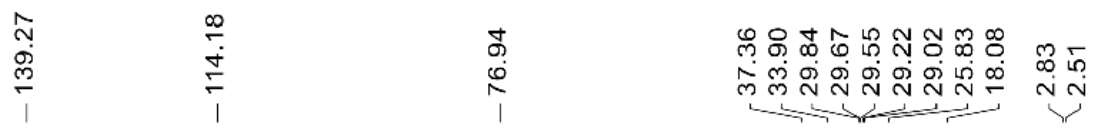

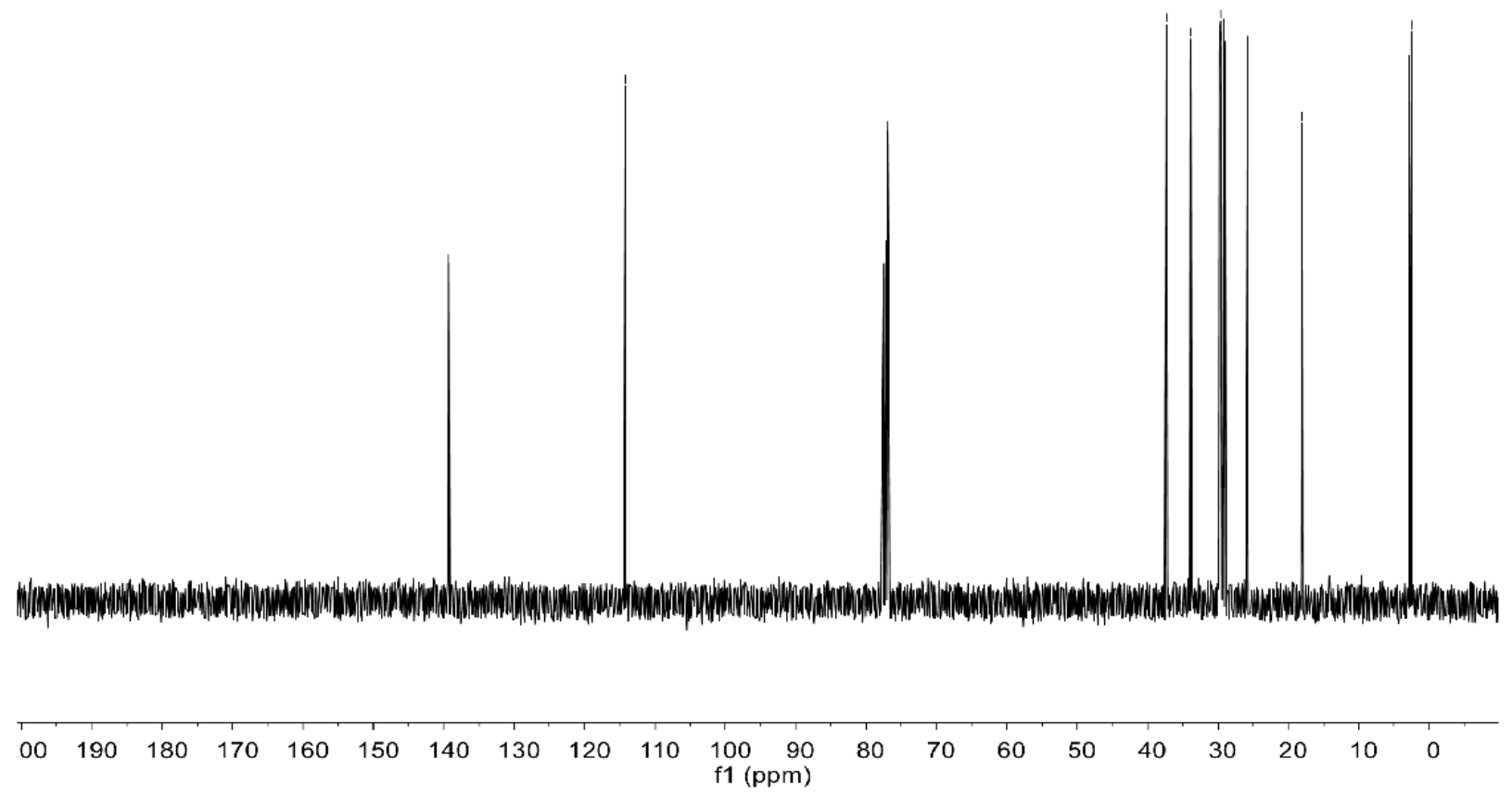




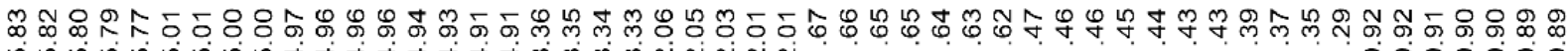
ம

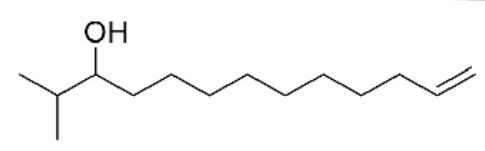

$1 \mathbf{x}$

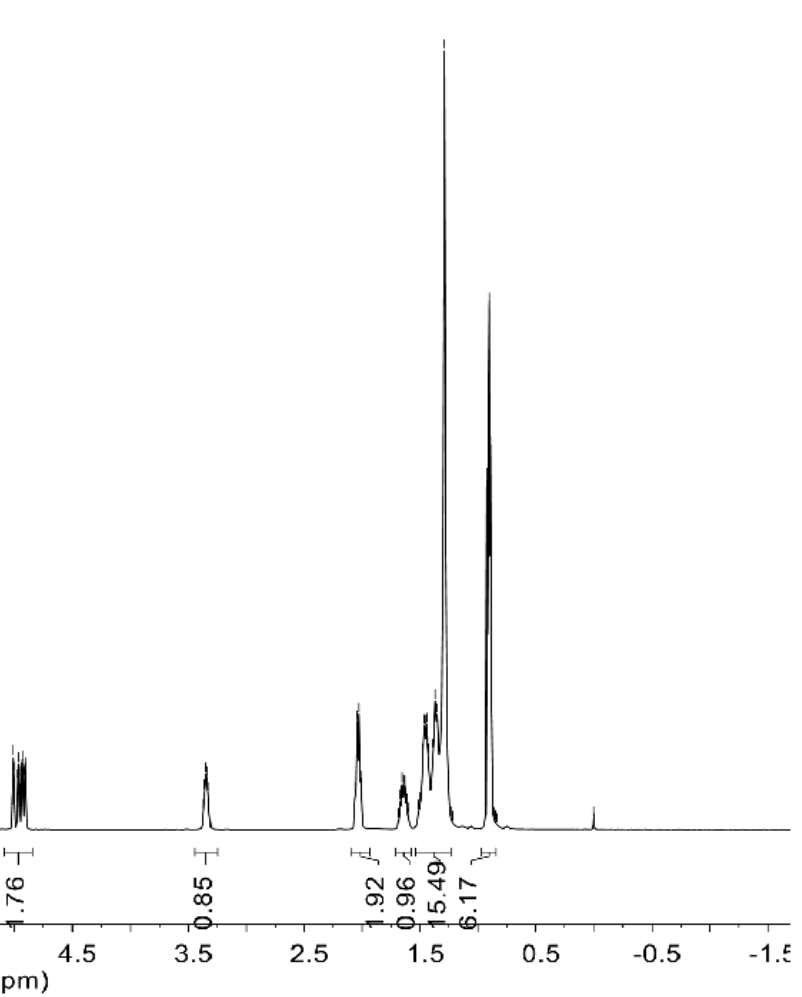

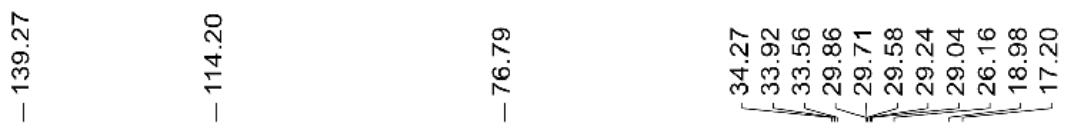

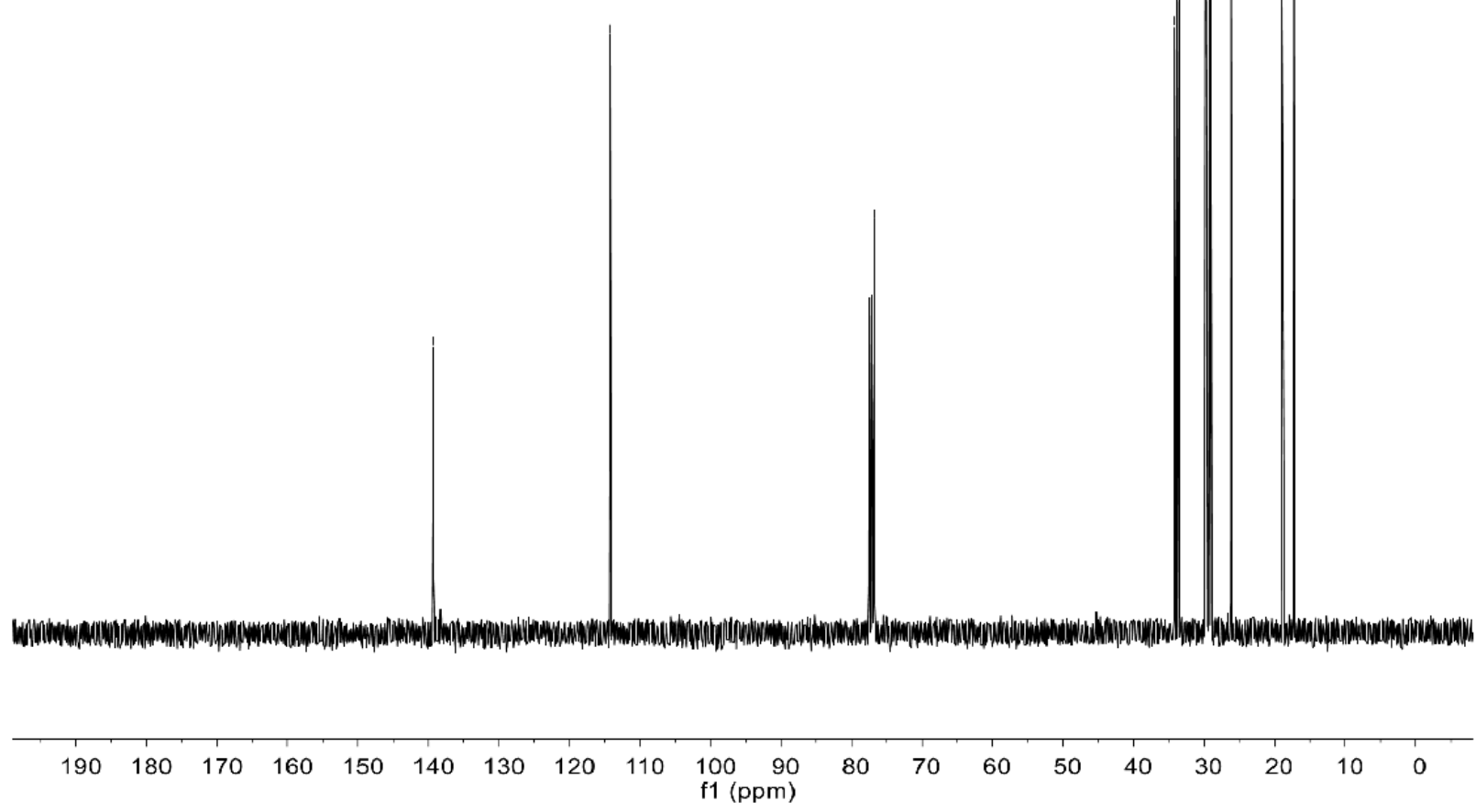




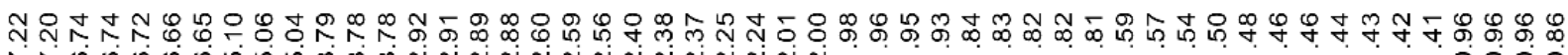
N $\dot{0} 0 \dot{0} \dot{0} \dot{0}$ ம

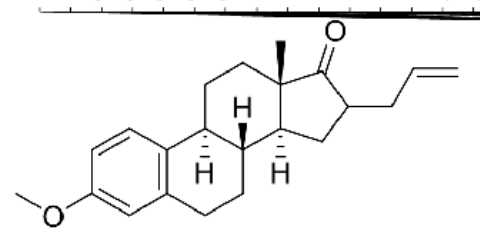

S1

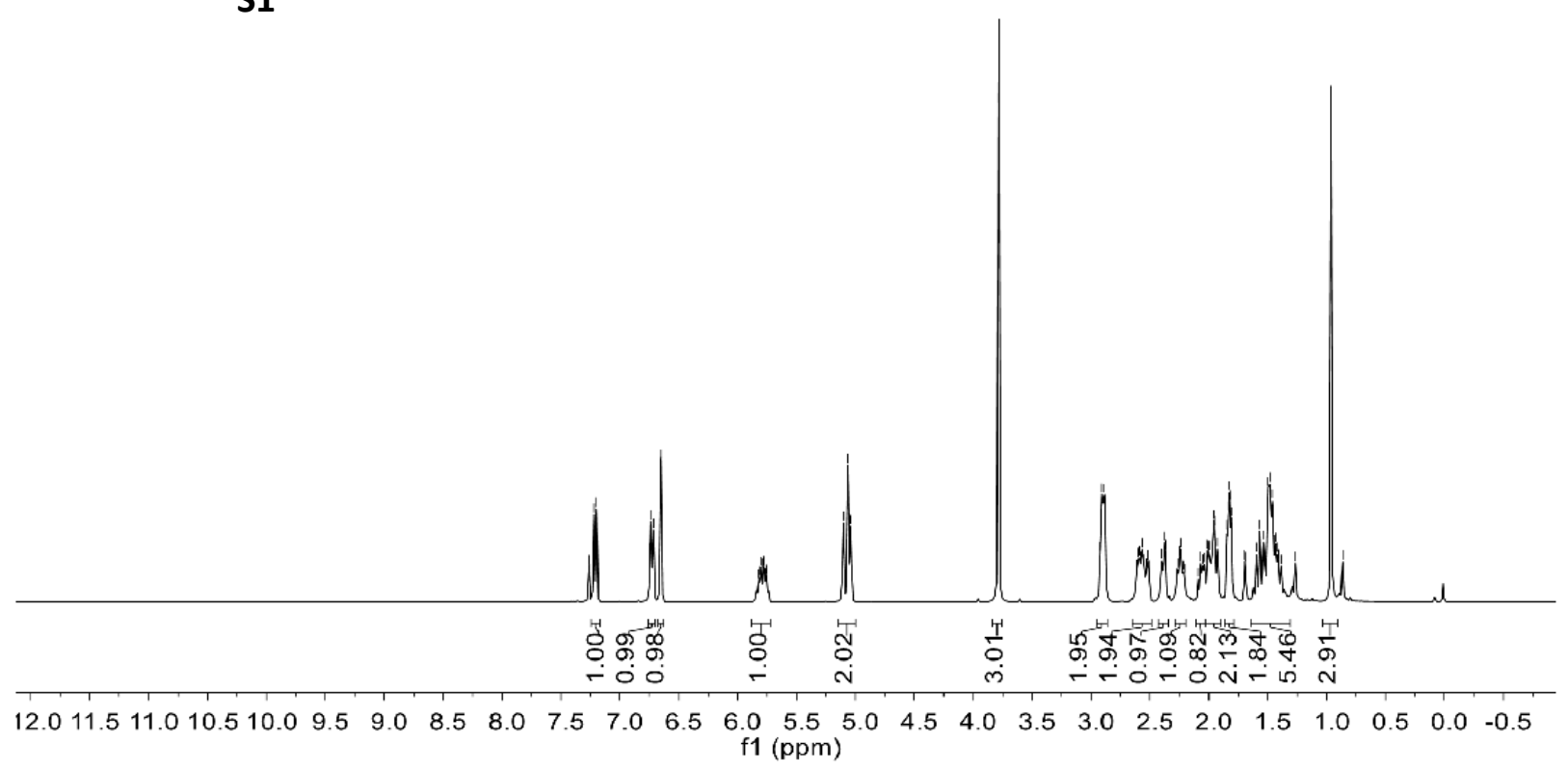

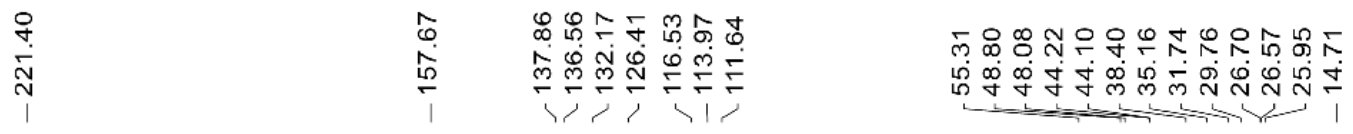

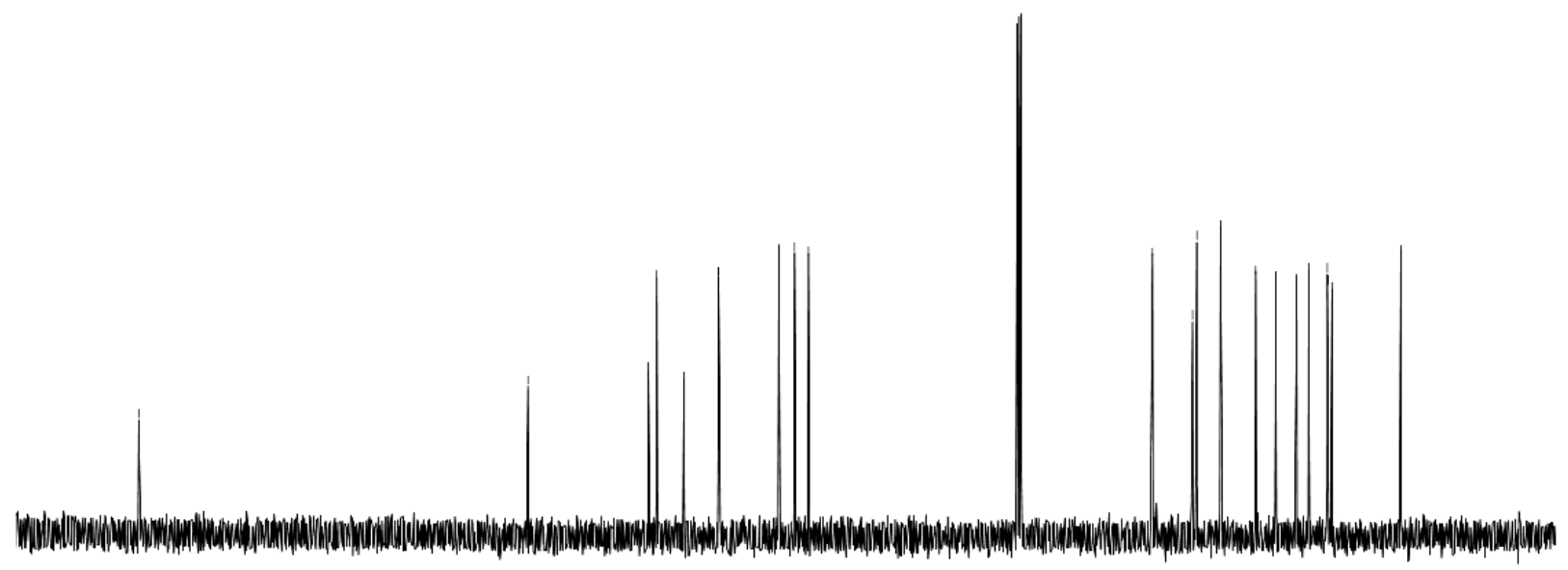

$\begin{array}{rllllllllllllllllllllllll}340 & 230 & 220 & 210 & 200 & 190 & 180 & 170 & 160 & 150 & 140 & 130 & \begin{array}{c}120 \\ \text { f1 }(\mathrm{ppm})\end{array} & 110 & 90 & 80 & 70 & 60 & 50 & 40 & 30 & 20 & 10 & 0 & -1\end{array}$ 


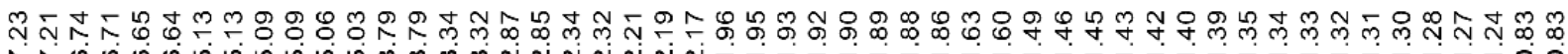

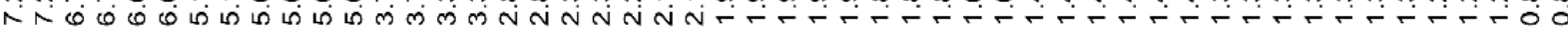<smiles>C=CCC1CC2CCc3cc(OC)ccc3C2CCC1C</smiles>

$1 a b$
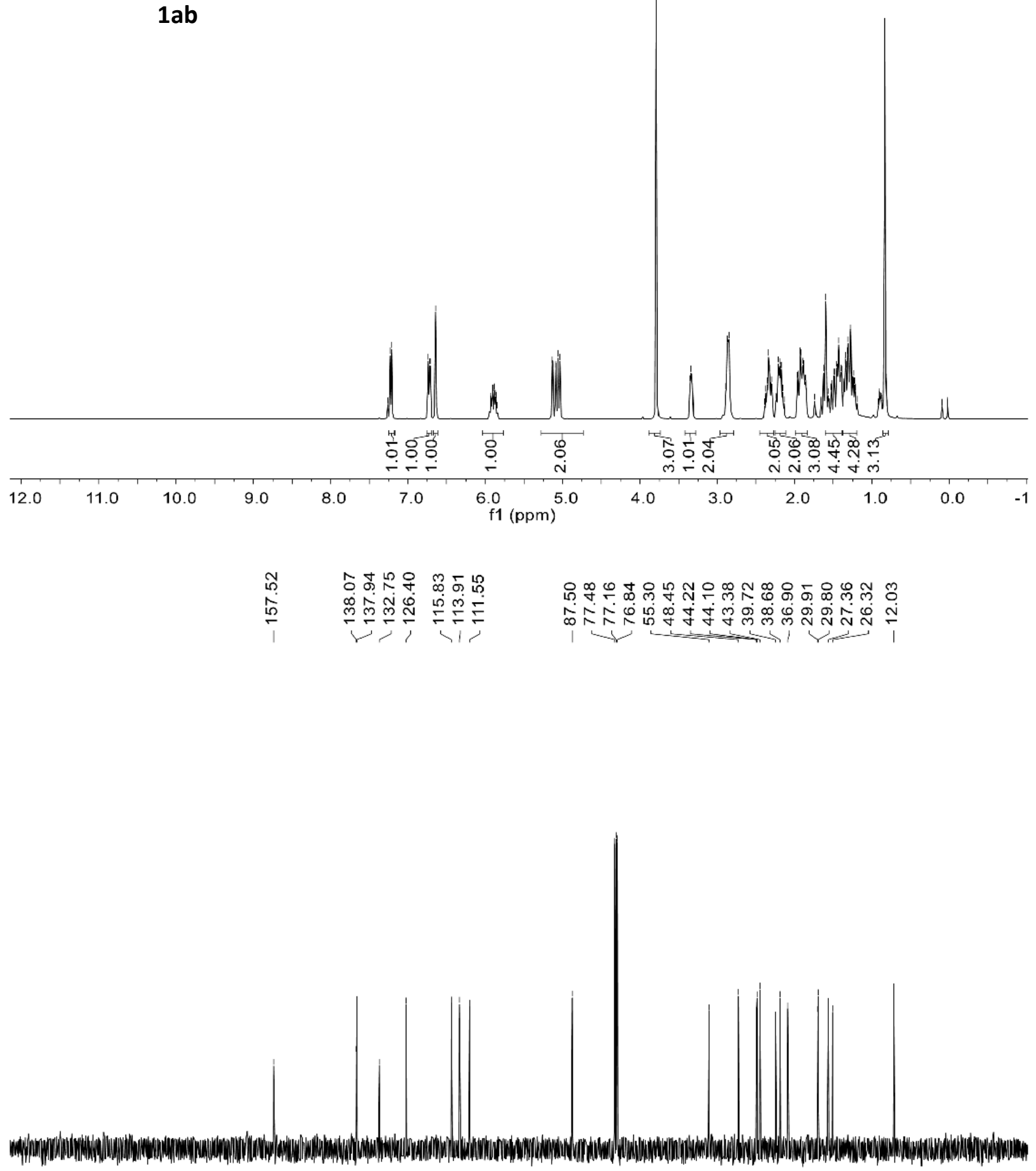

$\begin{array}{llllllllllllllllllllll}210 & 200 & 190 & 180 & 170 & 160 & 150 & 140 & 130 & 120 & \begin{array}{c}110 \\ \mathrm{f} 1\end{array}(\mathrm{ppm})\end{array}$ 
స్లెల ம.<smiles>C=CCCC(=O)C1CCC2[C@H]3CC=C4CC(OC(C)C)CC[C@]4(C)[C@H]3CC[C@@]12C</smiles>

S4

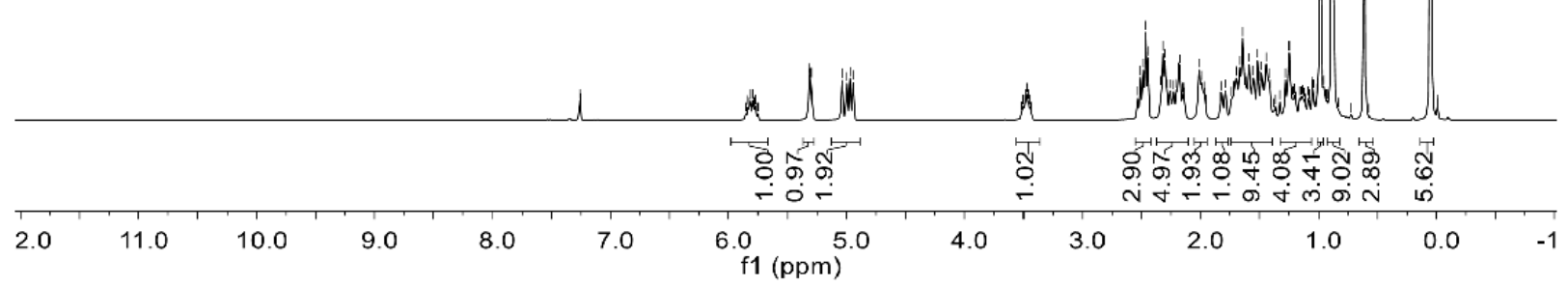

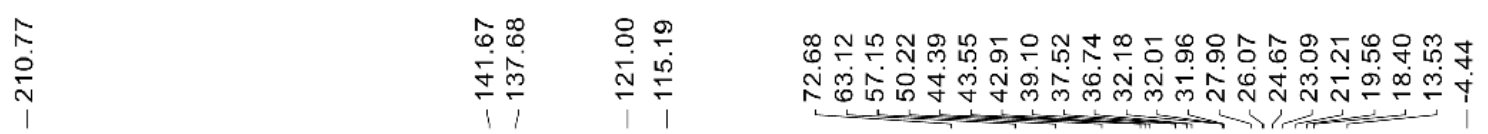

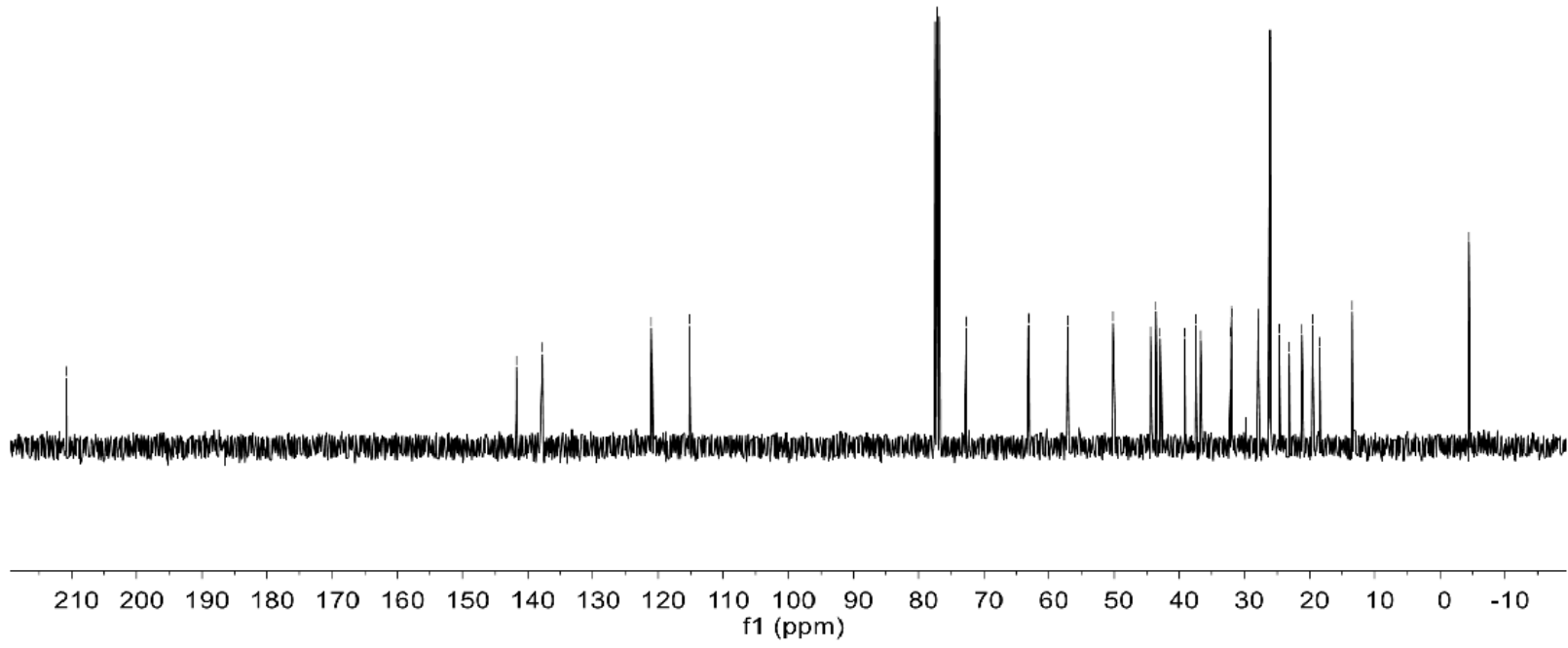




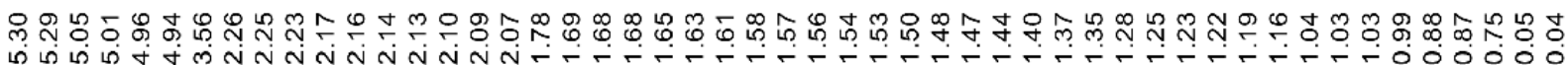<smiles>C=CCCC1CCC2C3CC=C4CC(OC(C)C)CCC4(C)C3CCC2(C)C1CCCC</smiles>

$1 \mathrm{ac}$

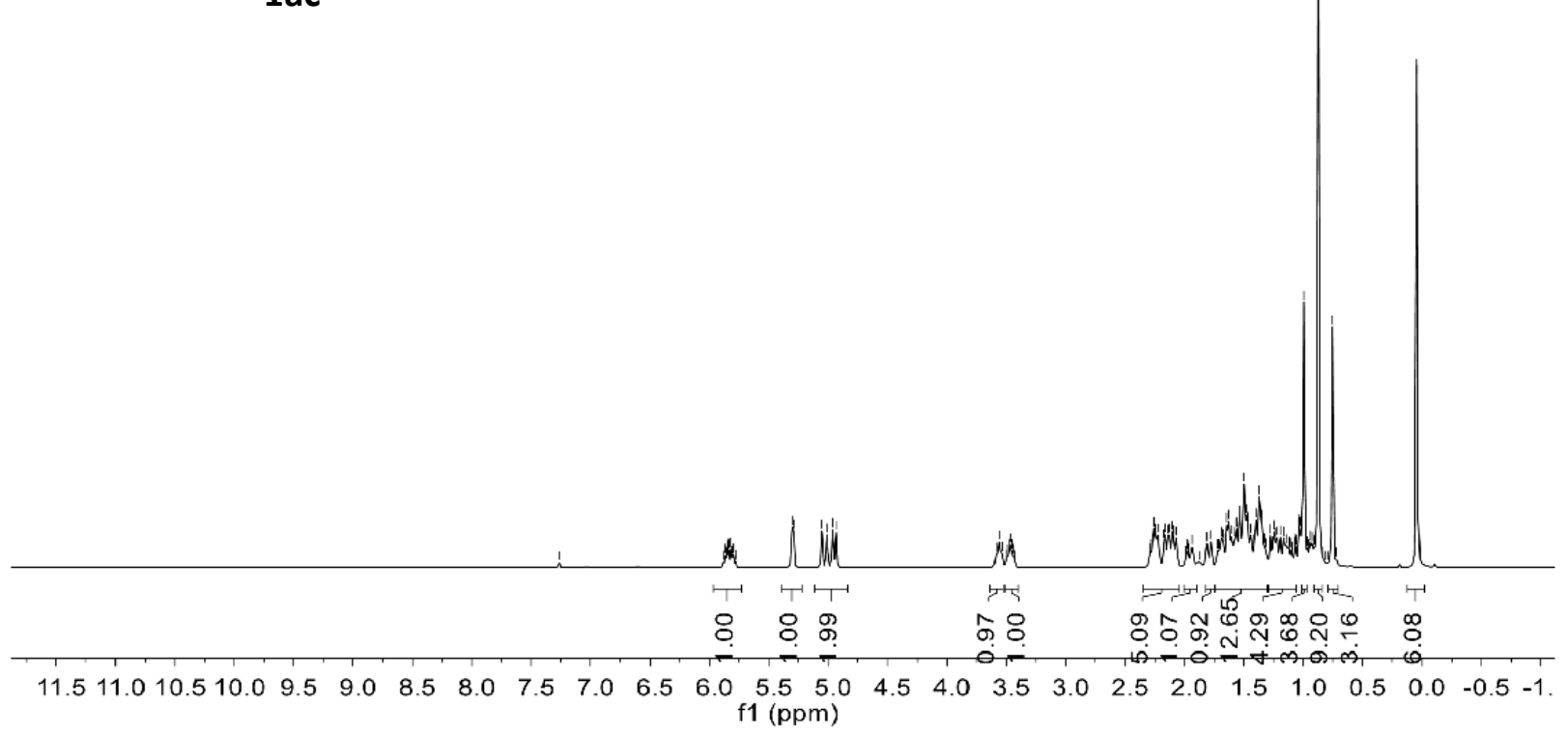

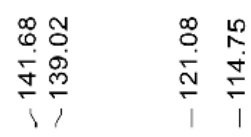

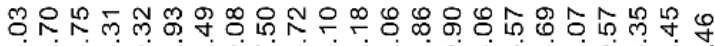

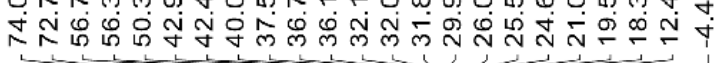

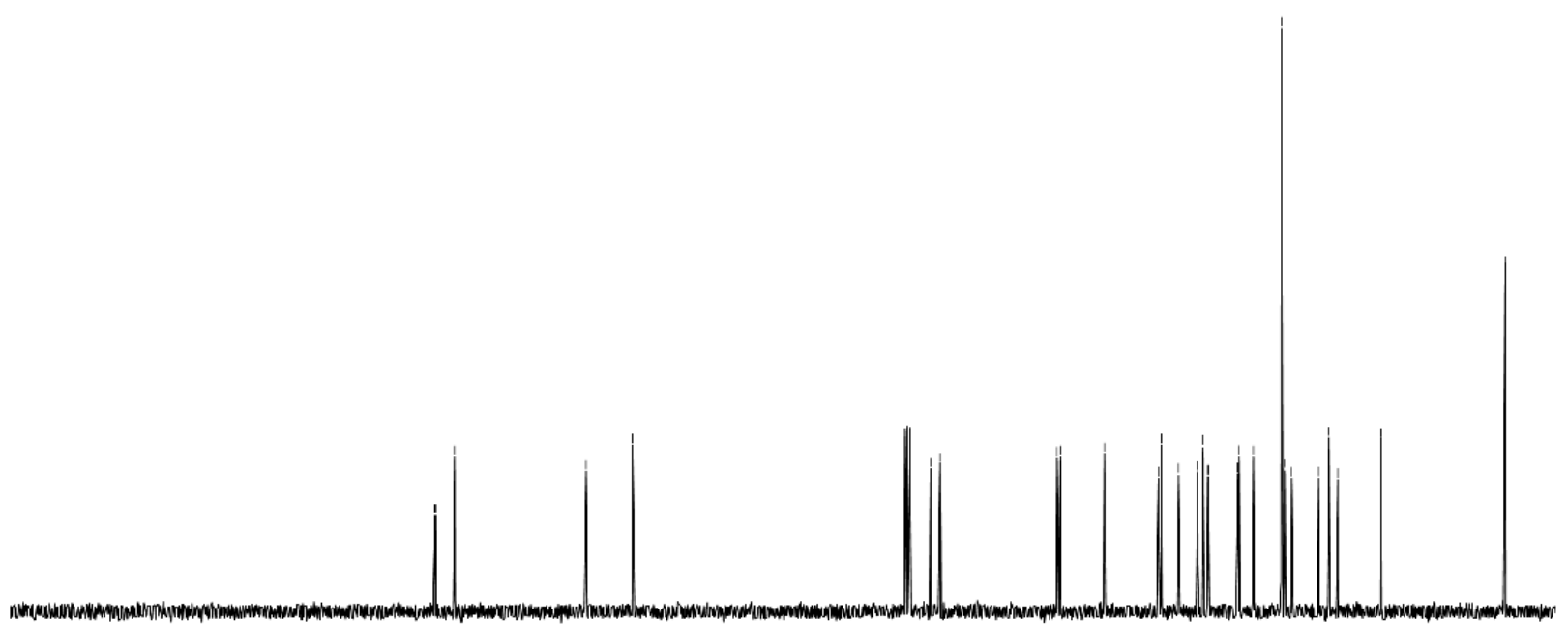

$\begin{array}{lllllllllllllllllllll}190 & 180 & 170 & 160 & 150 & 140 & 130 & 120 & 110 & \begin{array}{c}100 \\ f 1\end{array}(\mathrm{ppm}) & 90 & 80 & 70 & 60 & 50 & 40 & 30 & 20 & 10 & 0 & -1\end{array}$ 


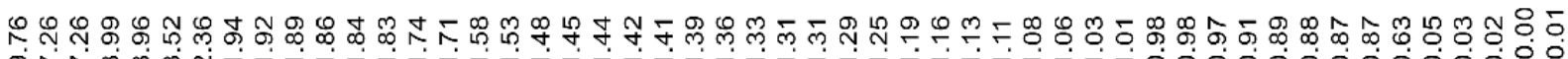

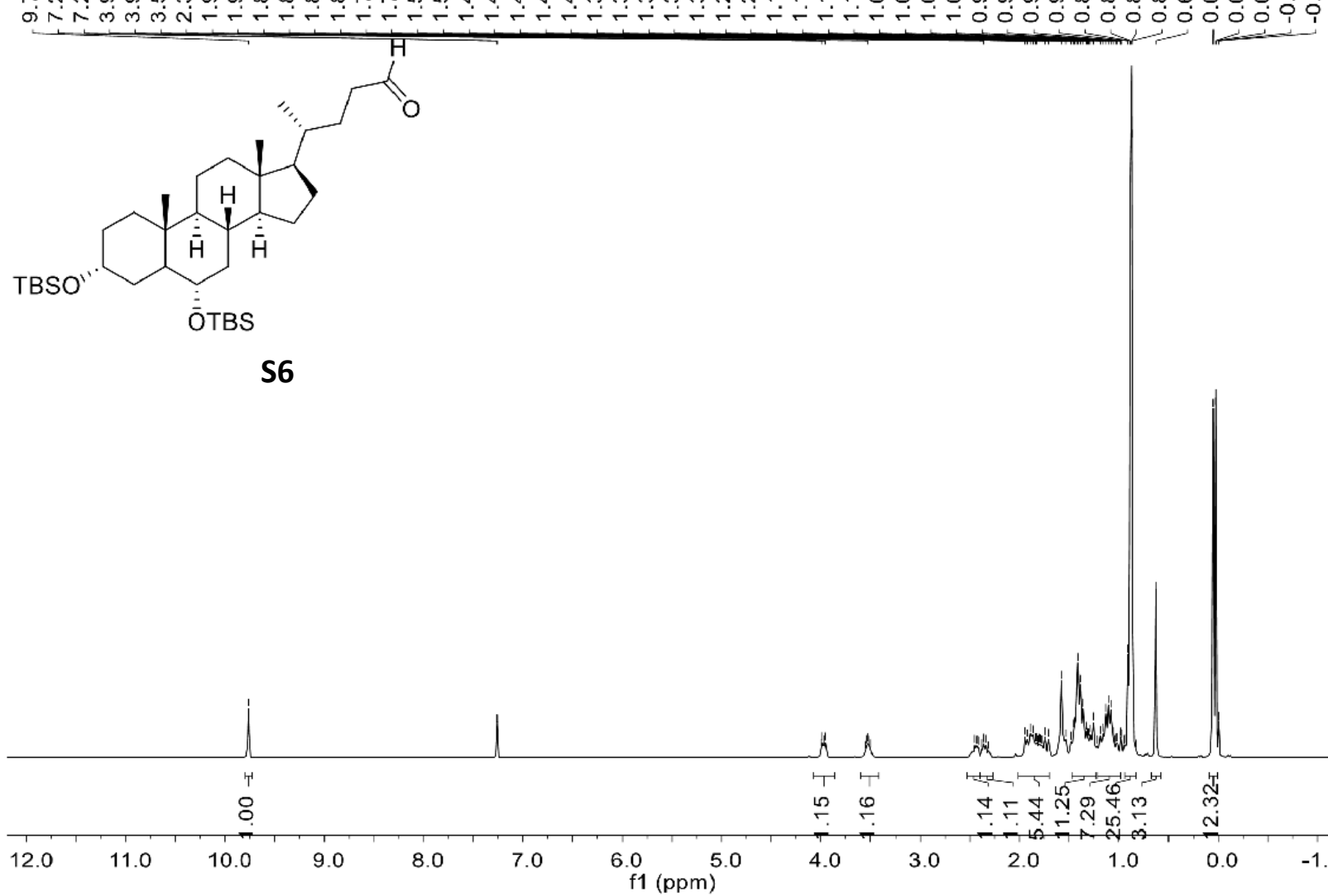

$\hat{m}$

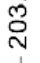

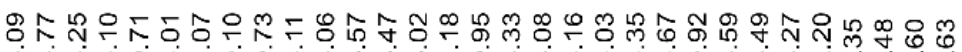

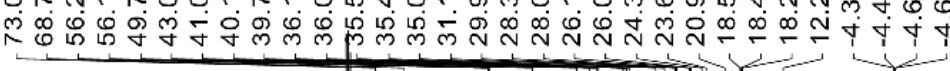

$\begin{array}{lllllllllllllllllllllll}210 & 200 & 190 & 180 & 170 & 160 & 150 & 140 & 130 & 120 & 110 \begin{array}{c}100 \\ \mathrm{f} 1 \\ (\mathrm{ppm})\end{array} & 90 & 80 & 70 & 60 & 50 & 40 & 30 & 20 & 10 & 0 & -10\end{array}$ 


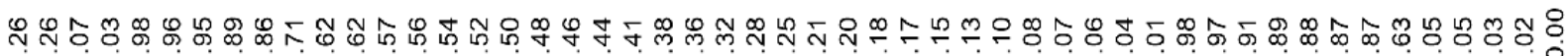

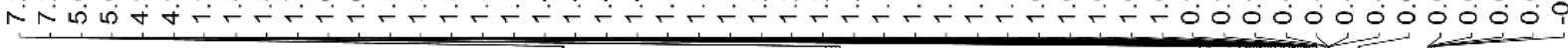

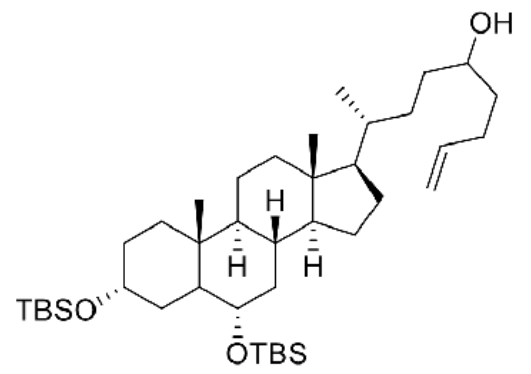

1ad

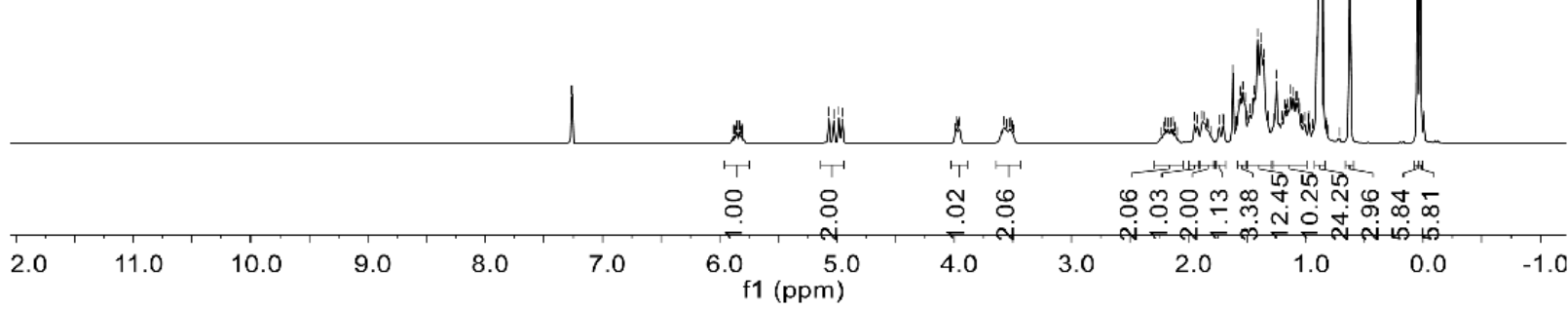

舟

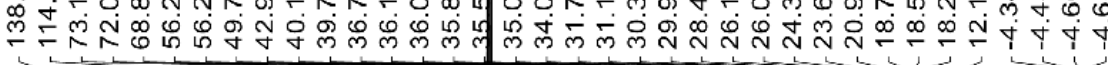

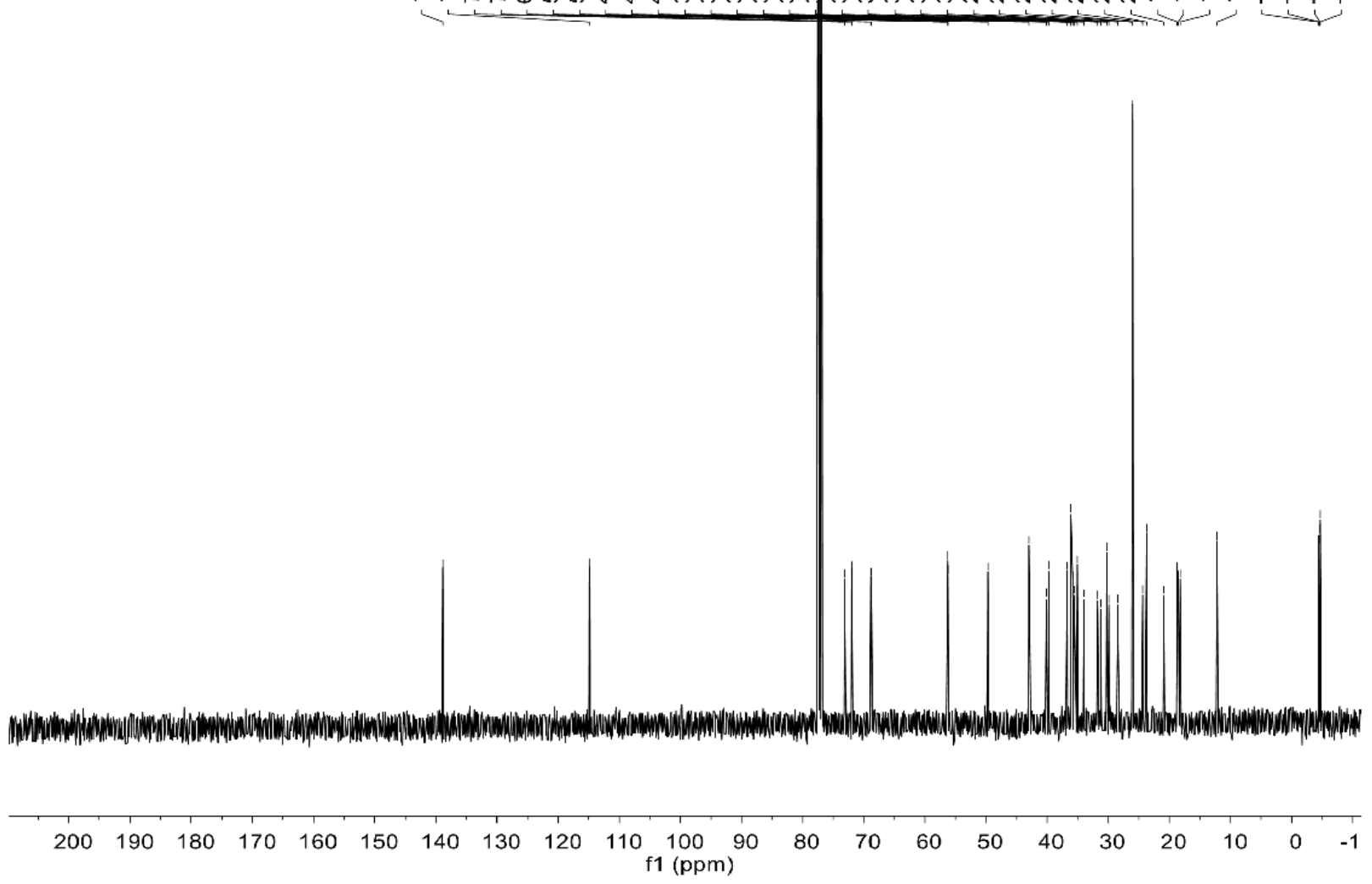




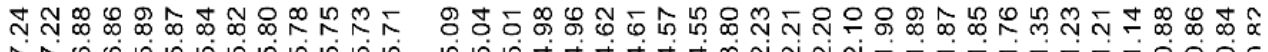<smiles>C=CCC(CCCC)=C(O)c1ccc(OC)cc1</smiles>

1 ae

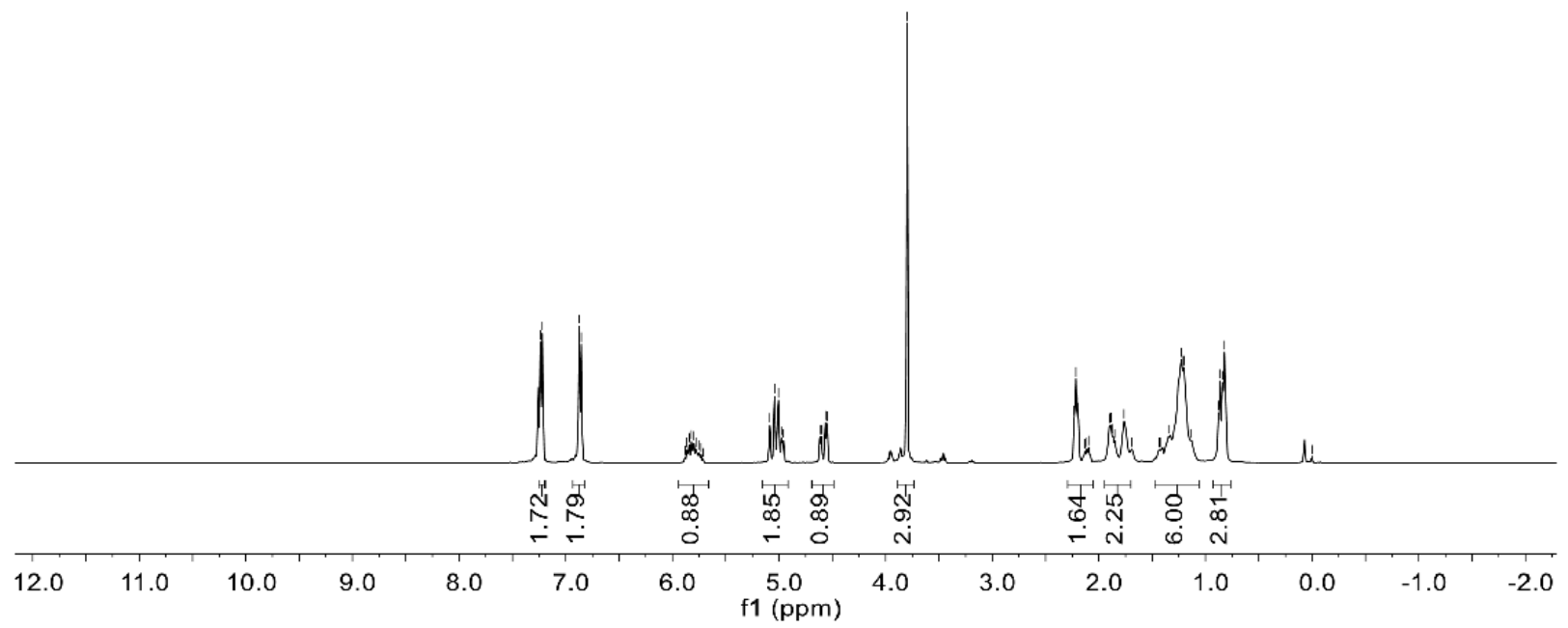

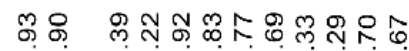

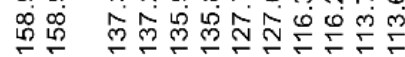

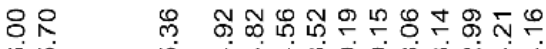

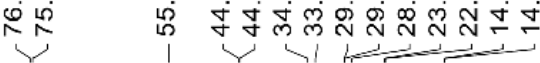

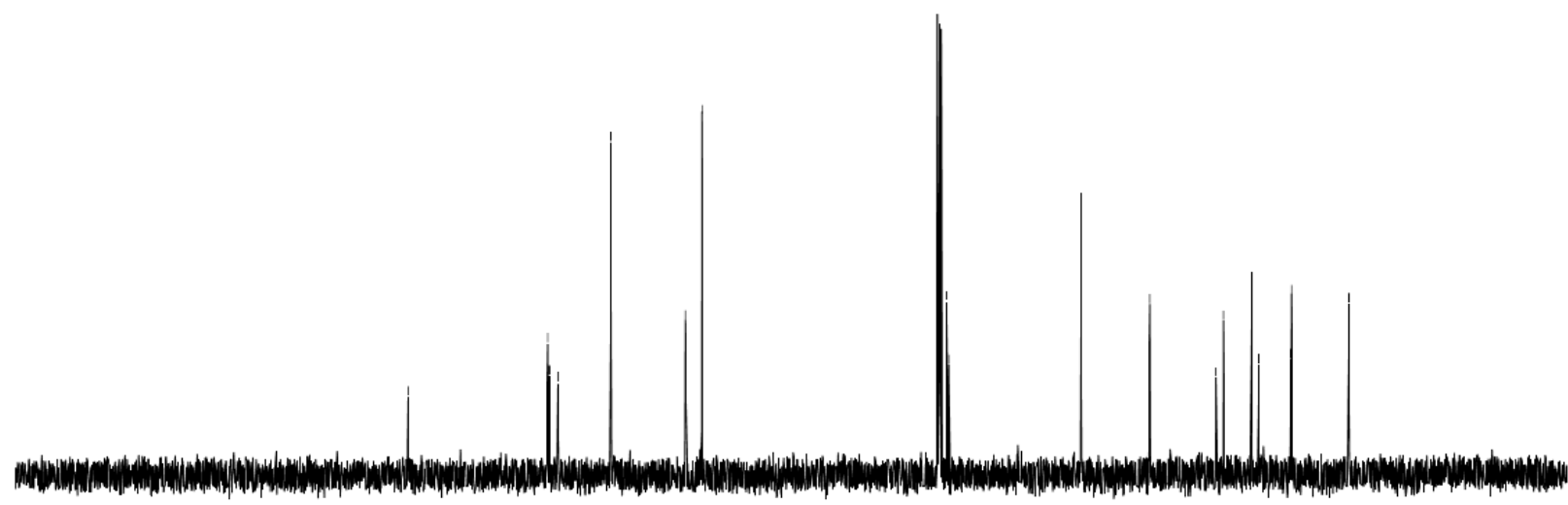

$\begin{array}{lllllllllllllllllllllll}210 & 200 & 190 & 180 & 170 & 160 & 150 & 140 & 130 & 120 & 110 & 100 & 90 & 80 & 70 & 60 & 50 & 40 & 30 & 20 & 10 & 0 & -10\end{array}$ 

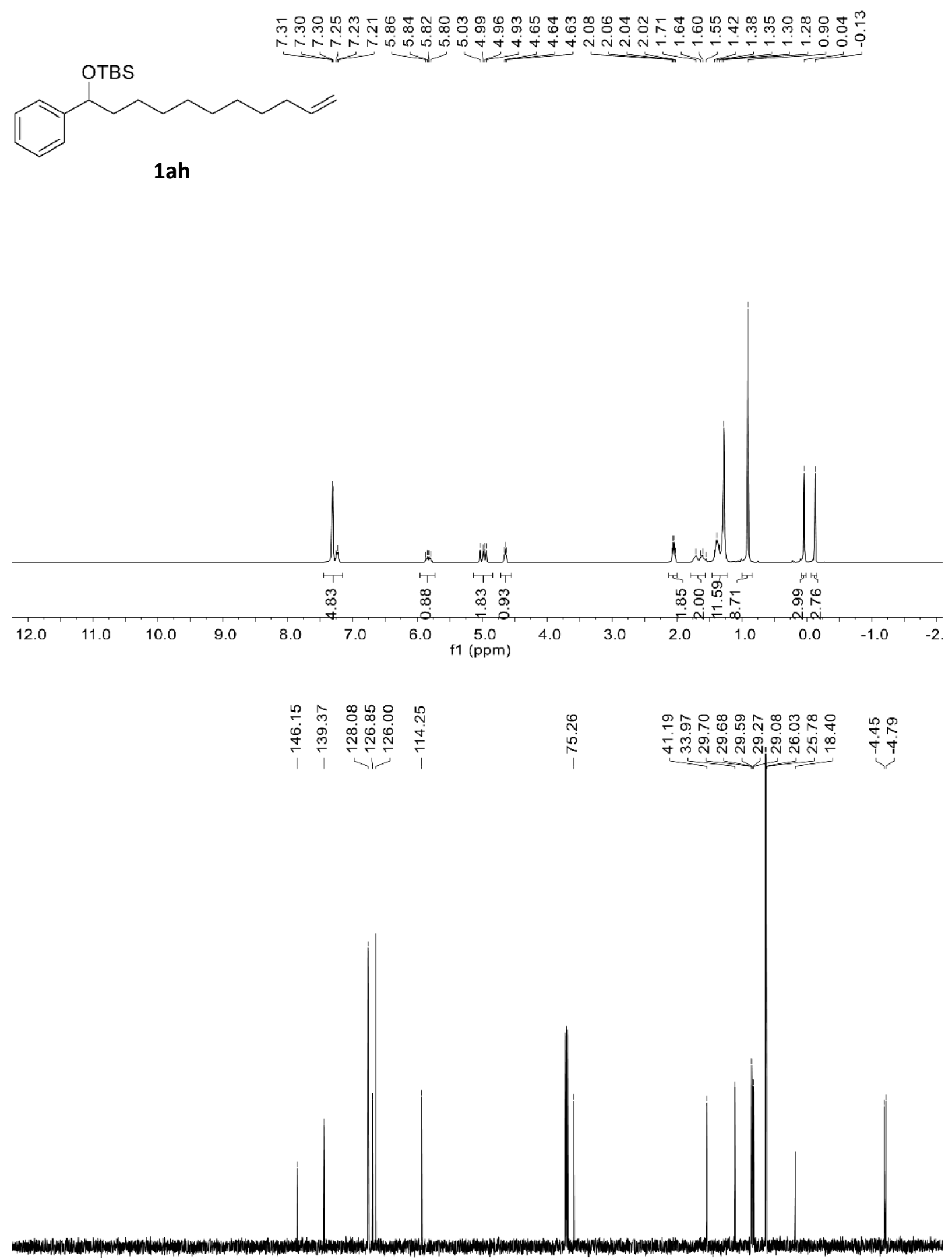

$\begin{array}{lllllllllllllllllllllll}210 & 200 & 190 & 180 & 170 & 160 & 150 & 140 & 130 & 120 & 110 & 100 & 90 & 80 & 70 & 60 & 50 & 40 & 30 & 20 & 10 & 0 & -10\end{array}$ 
<smiles>C=CCCC(O)(O)c1ccc(OC)cc1</smiles>

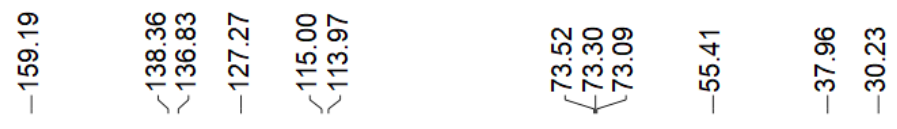

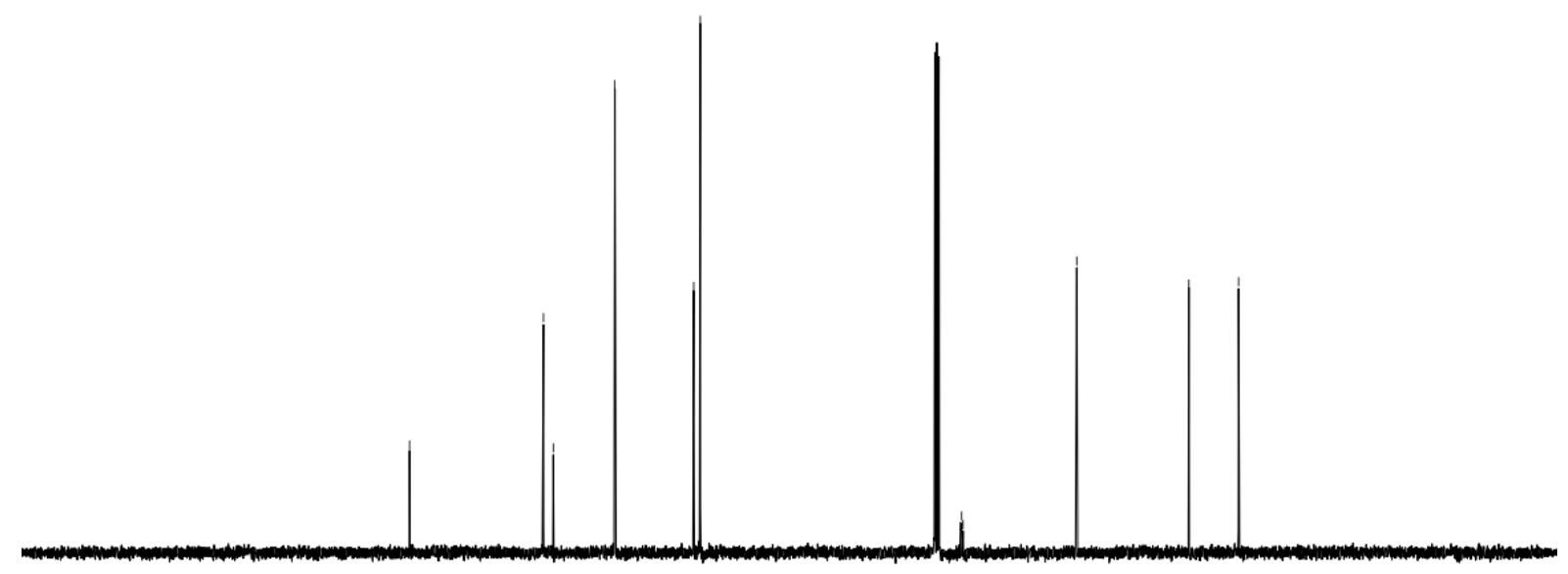

$\begin{array}{lllllllllllllllllllllll}210 & 200 & 190 & 180 & 170 & 160 & 150 & 140 & 130 & 120 & 110 & 100 & 90 & 80 & 70 & 60 & 50 & 40 & 30 & 20 & 10 & 0 & -10\end{array}$ 


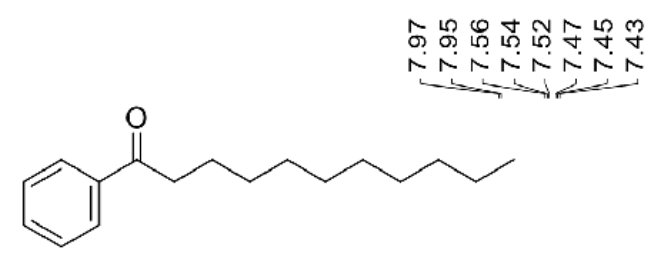

๙ิ

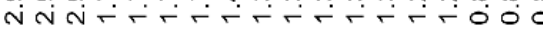

$2 a$
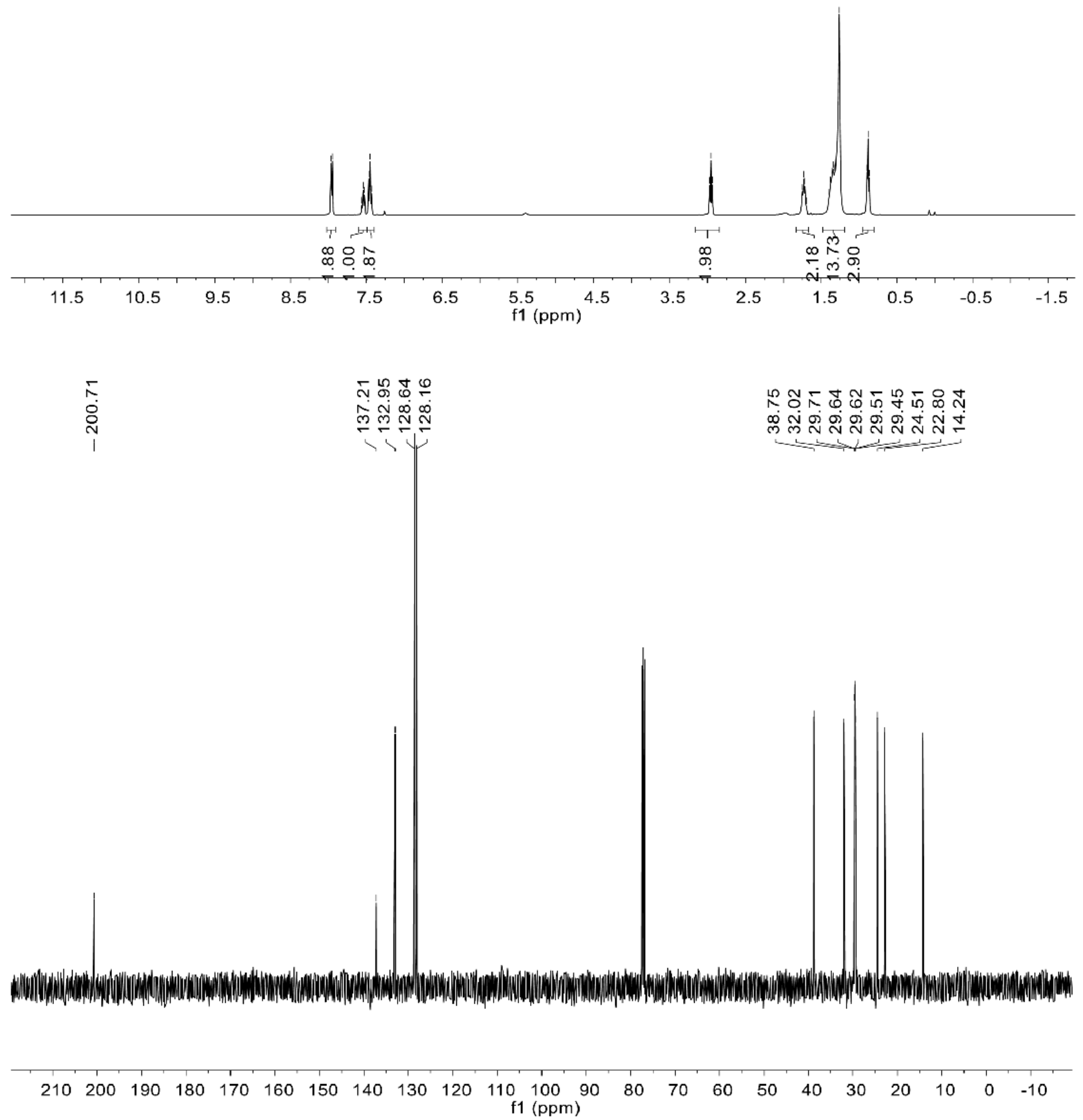


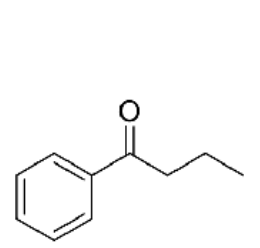

$2 b$

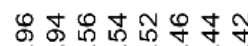

NNNNNN

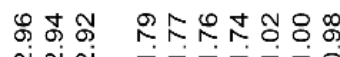

$\sqrt{4 n}$
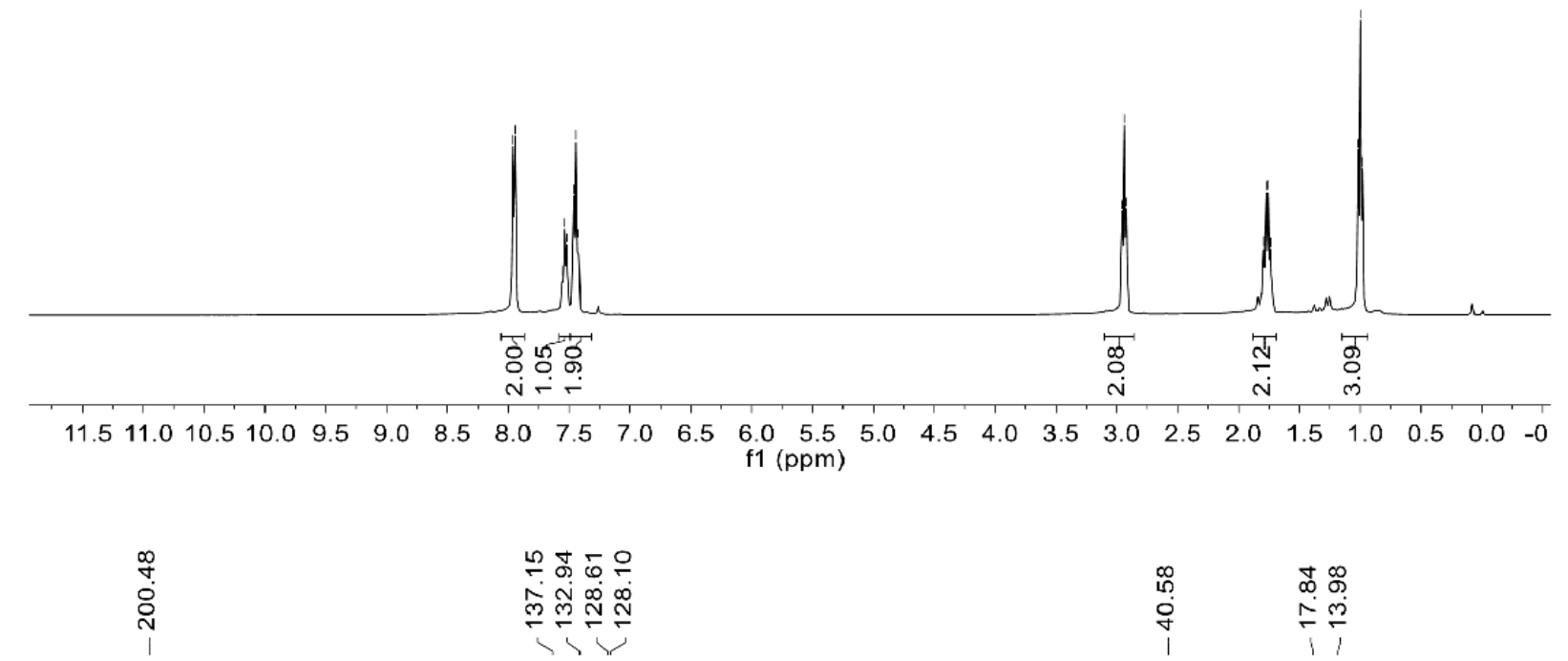

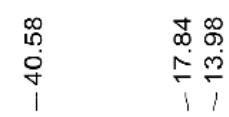

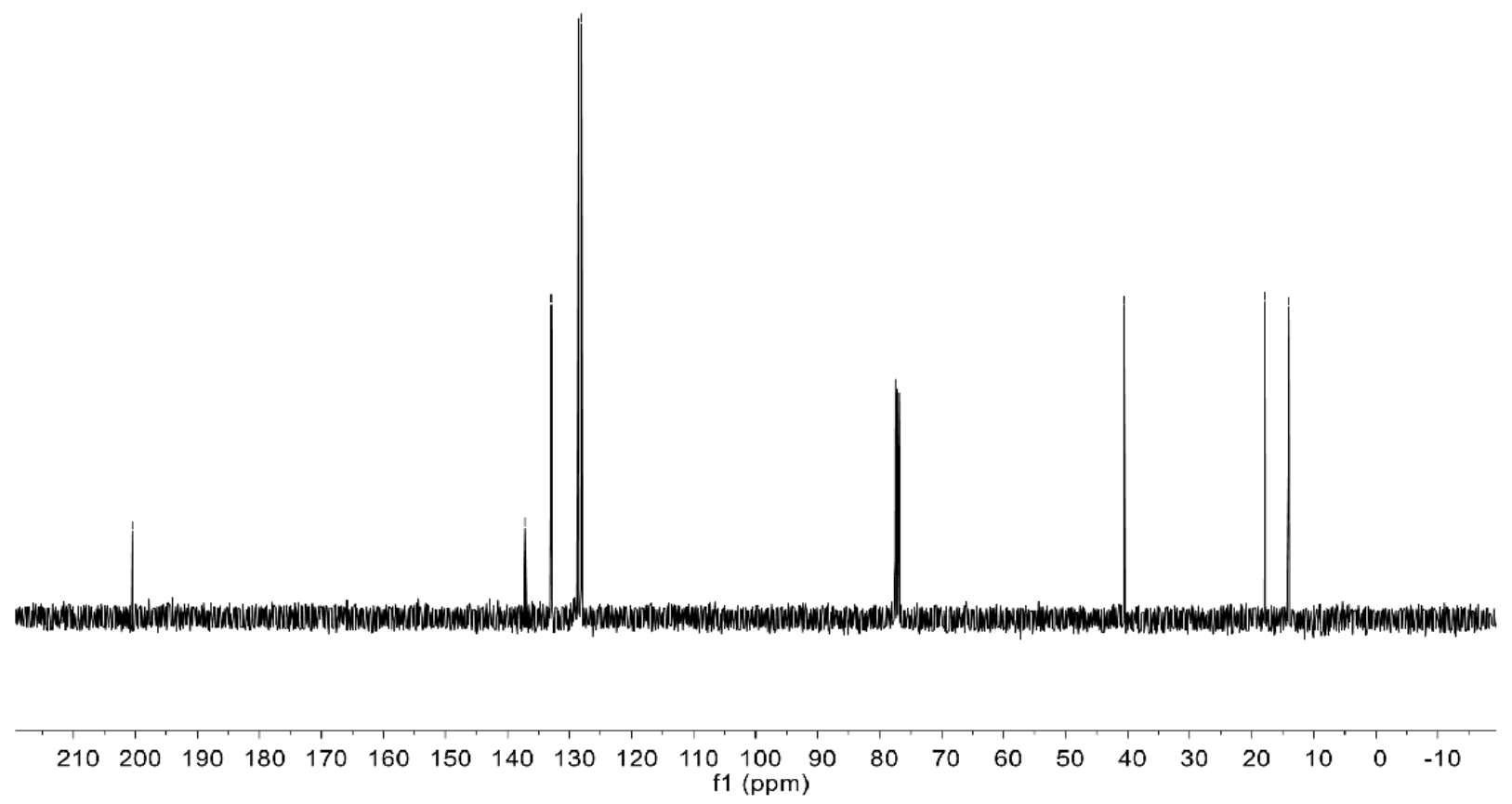



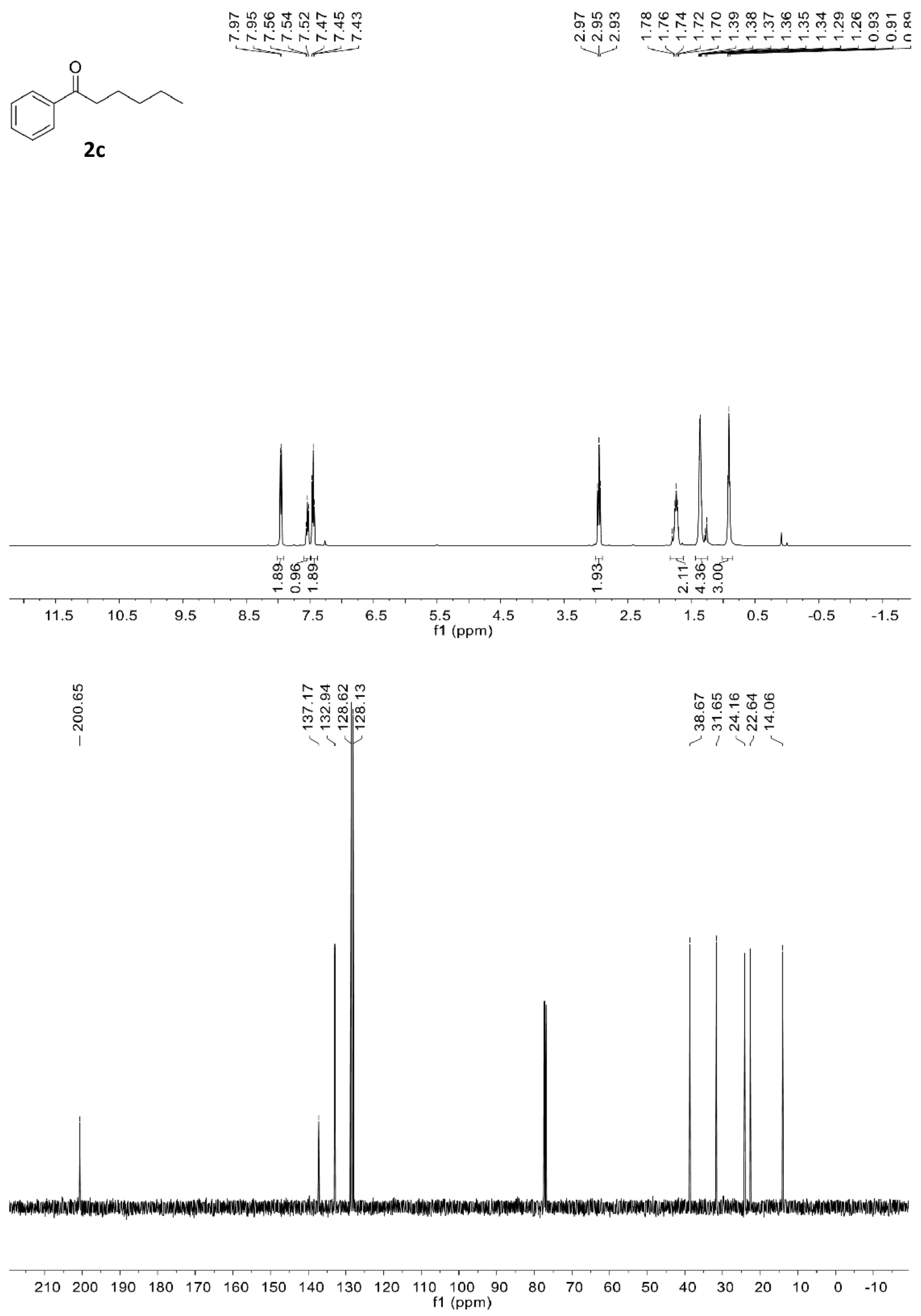


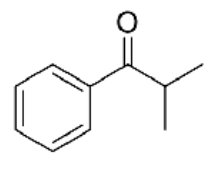

ติ่

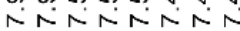

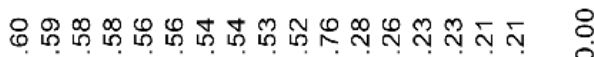

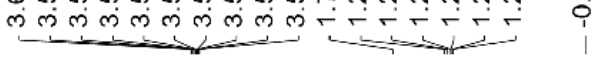

2d

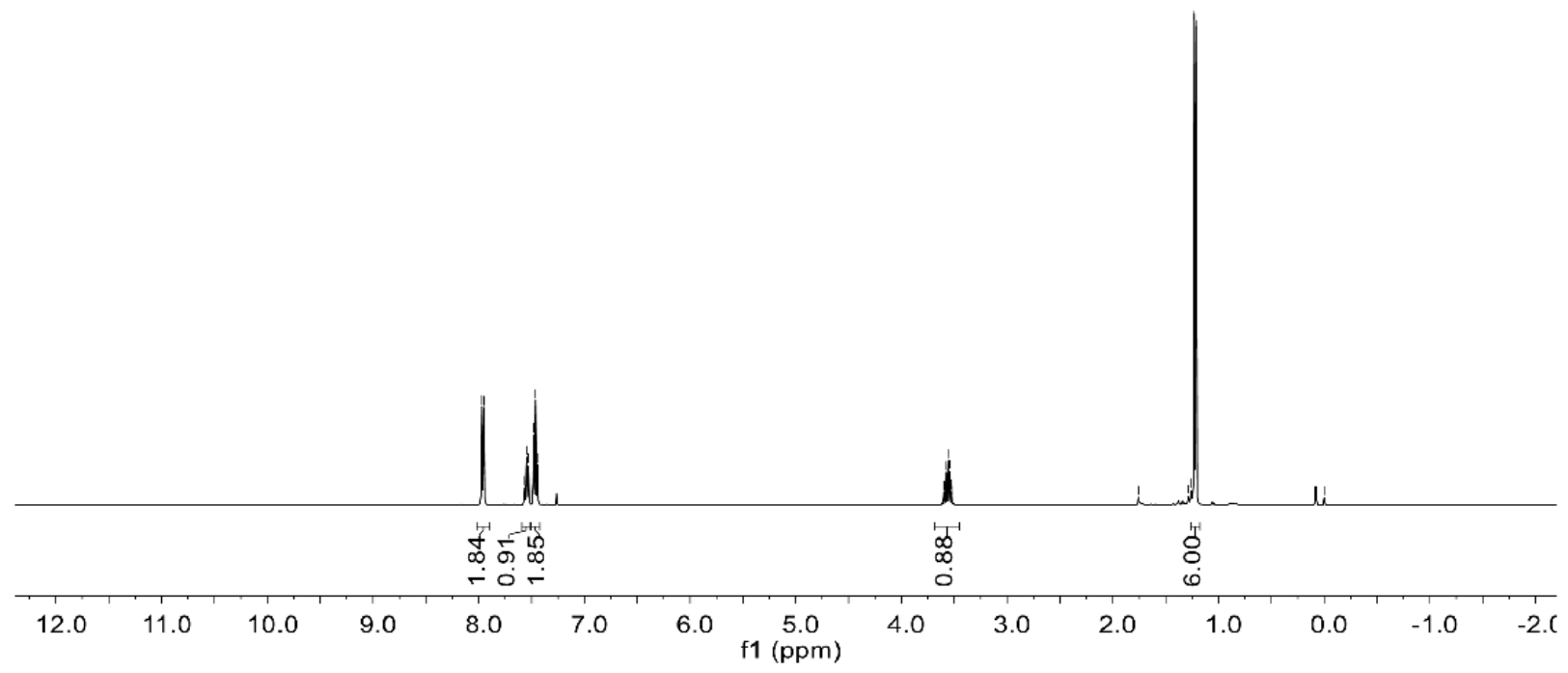

¿্ৰ

\section{잉ㄷ}

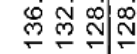

난

点

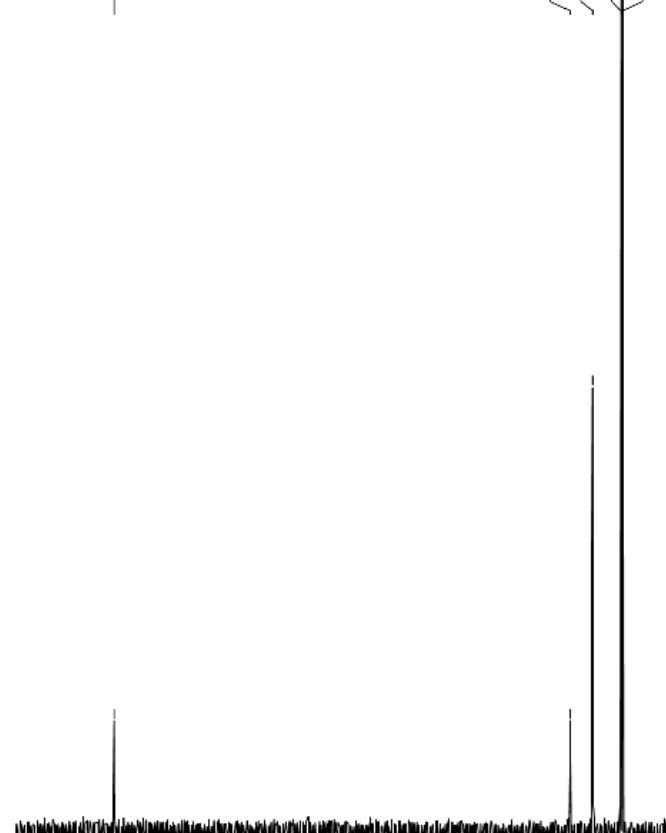

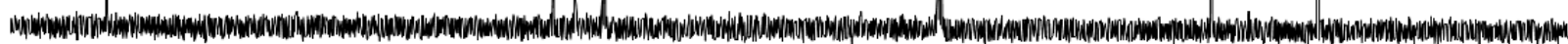

$\begin{array}{lllllllllllllllllllllll}210 & 200 & 190 & 180 & 170 & 160 & 150 & 140 & 130 & 120 & 110 & 100 & 90 & 80 & 70 & 60 & 50 & 40 & 30 & 20 & 10 & 0 & -10\end{array}$ f1 $(\mathrm{ppm})$ 


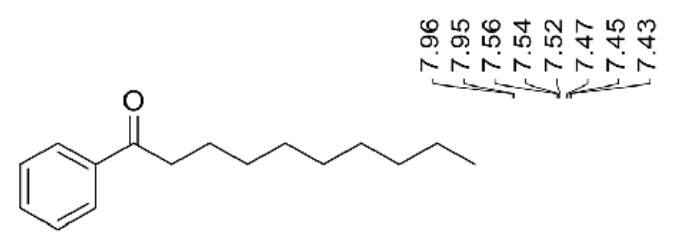

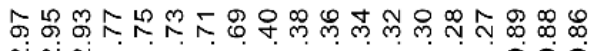

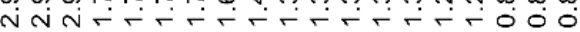

$2 e$

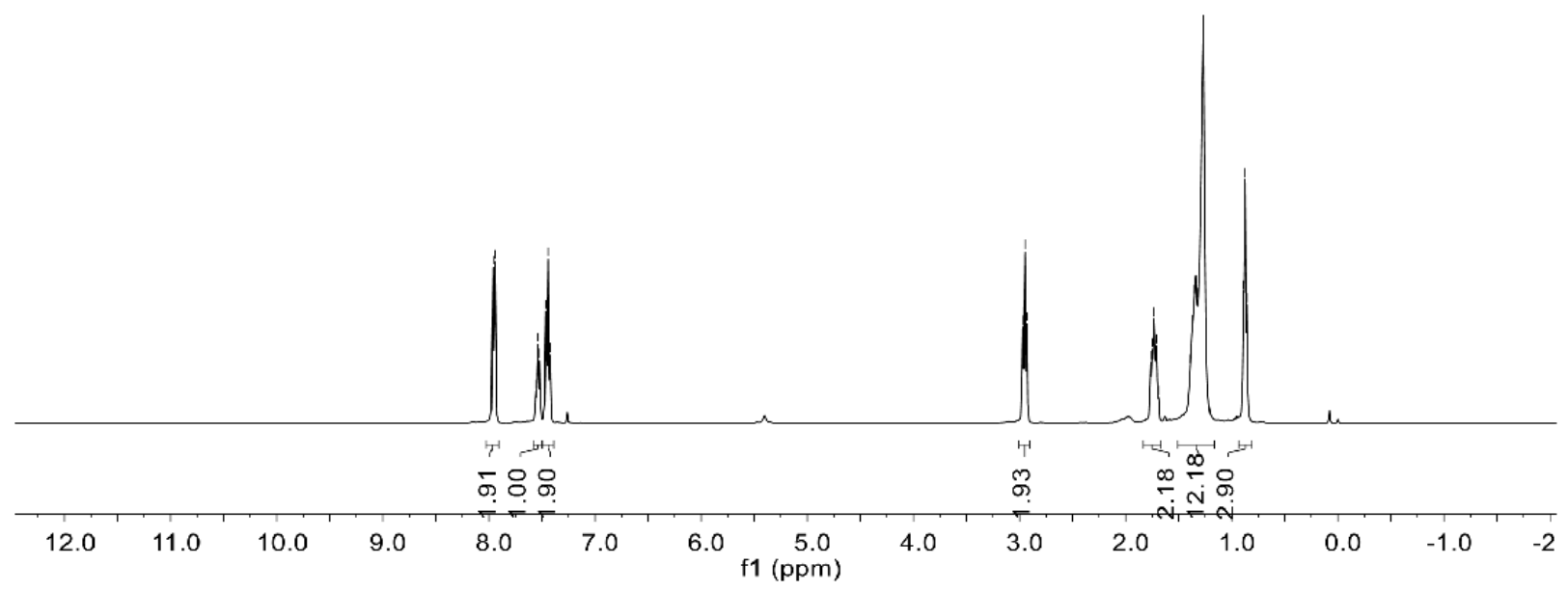

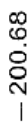

กู ๒ู ๒

商

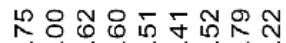

ભ लై

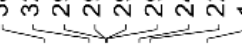

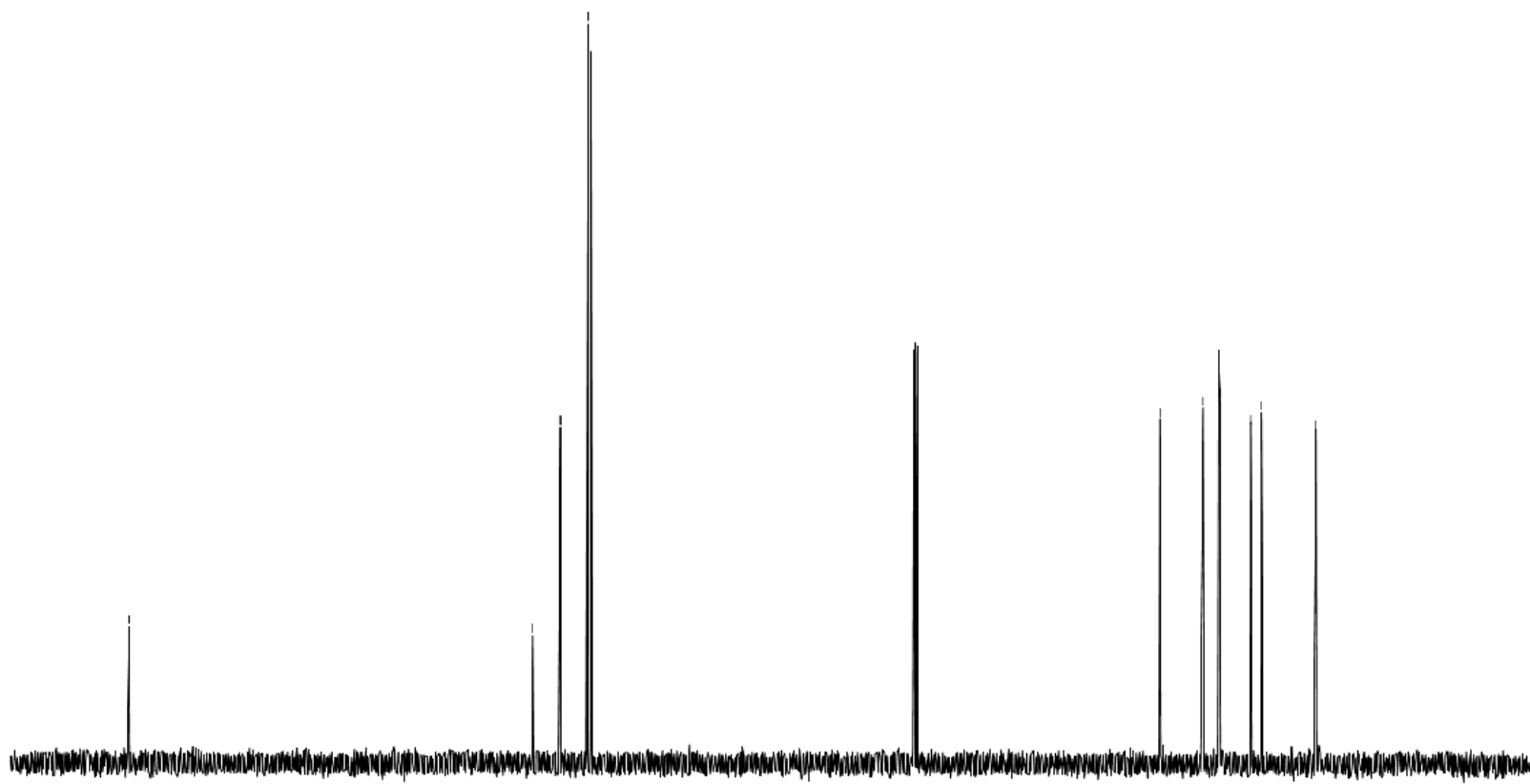

$\begin{array}{lllllllllllllllllllllll}210 & 200 & 190 & 180 & 170 & 160 & 150 & 140 & 130 & 120 & \begin{array}{c}110 \\ \mathrm{f} 1\end{array} \begin{array}{l}100 \\ (\mathrm{ppm})\end{array} & 90 & 80 & 70 & 60 & 50 & 40 & 30 & 20 & 10 & 0 & -10\end{array}$ 


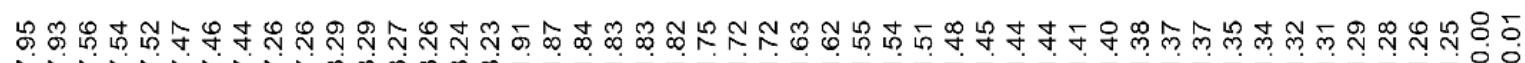

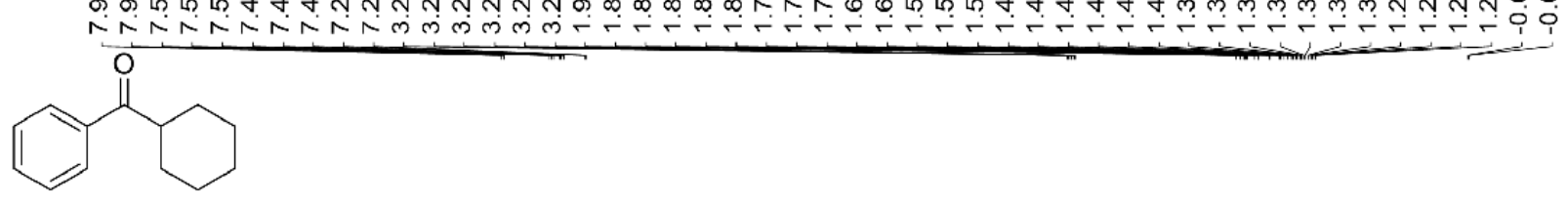

$2 f$

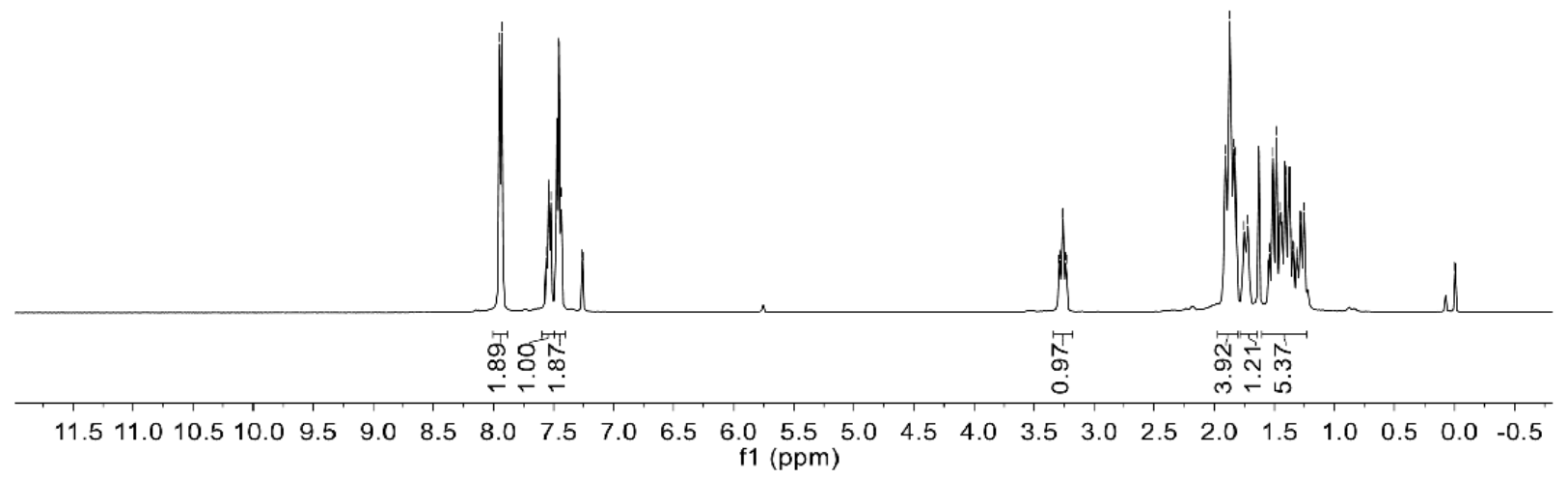

I

웅요

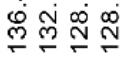

$\stackrel{0}{\infty}=5$

เ

I

-

$\checkmark v$

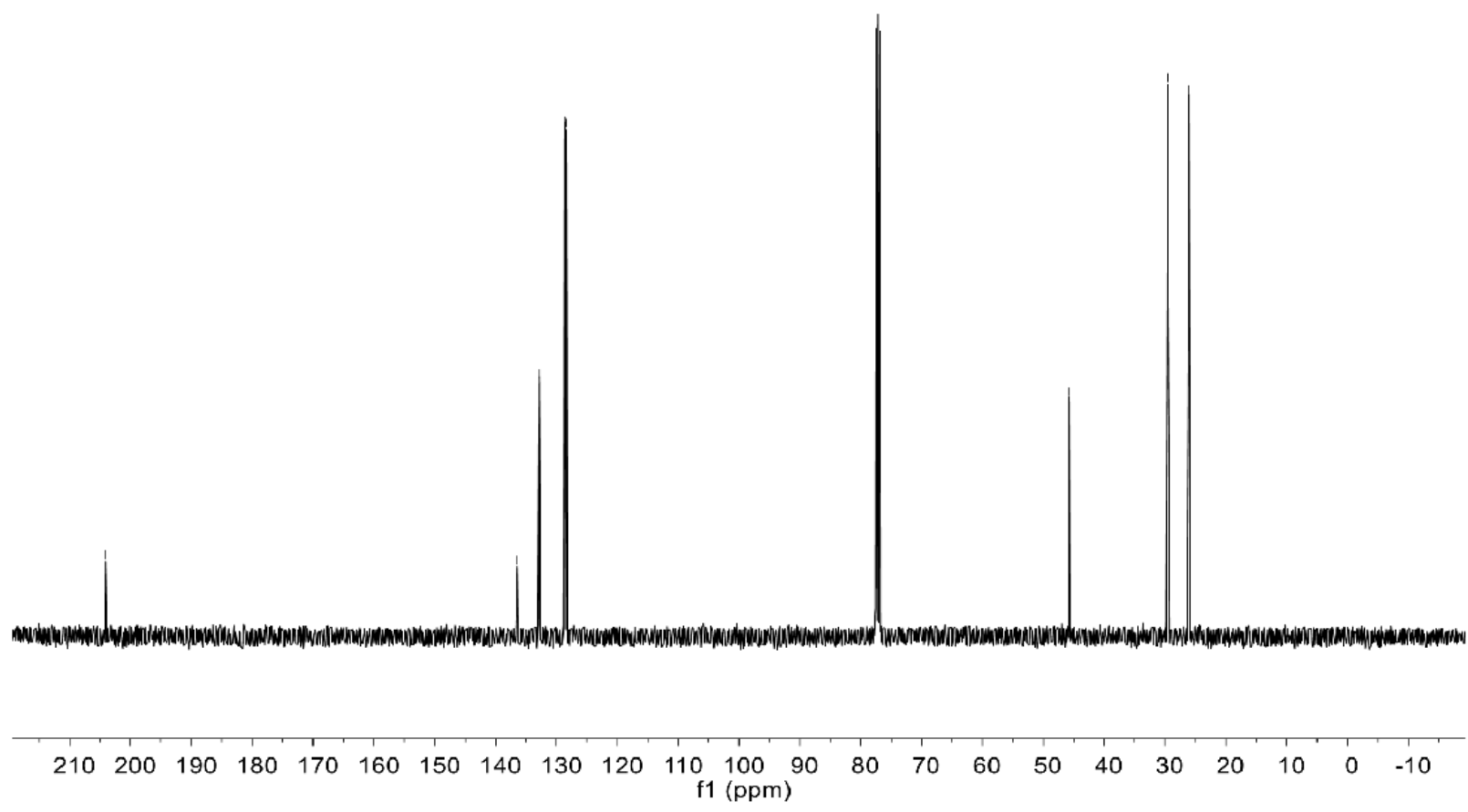




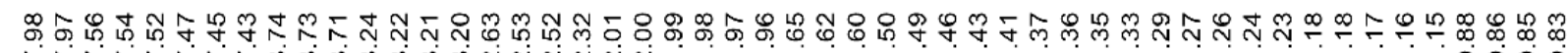

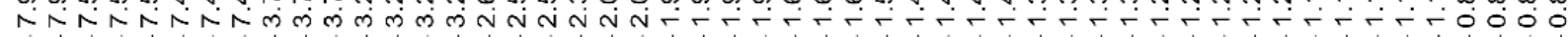
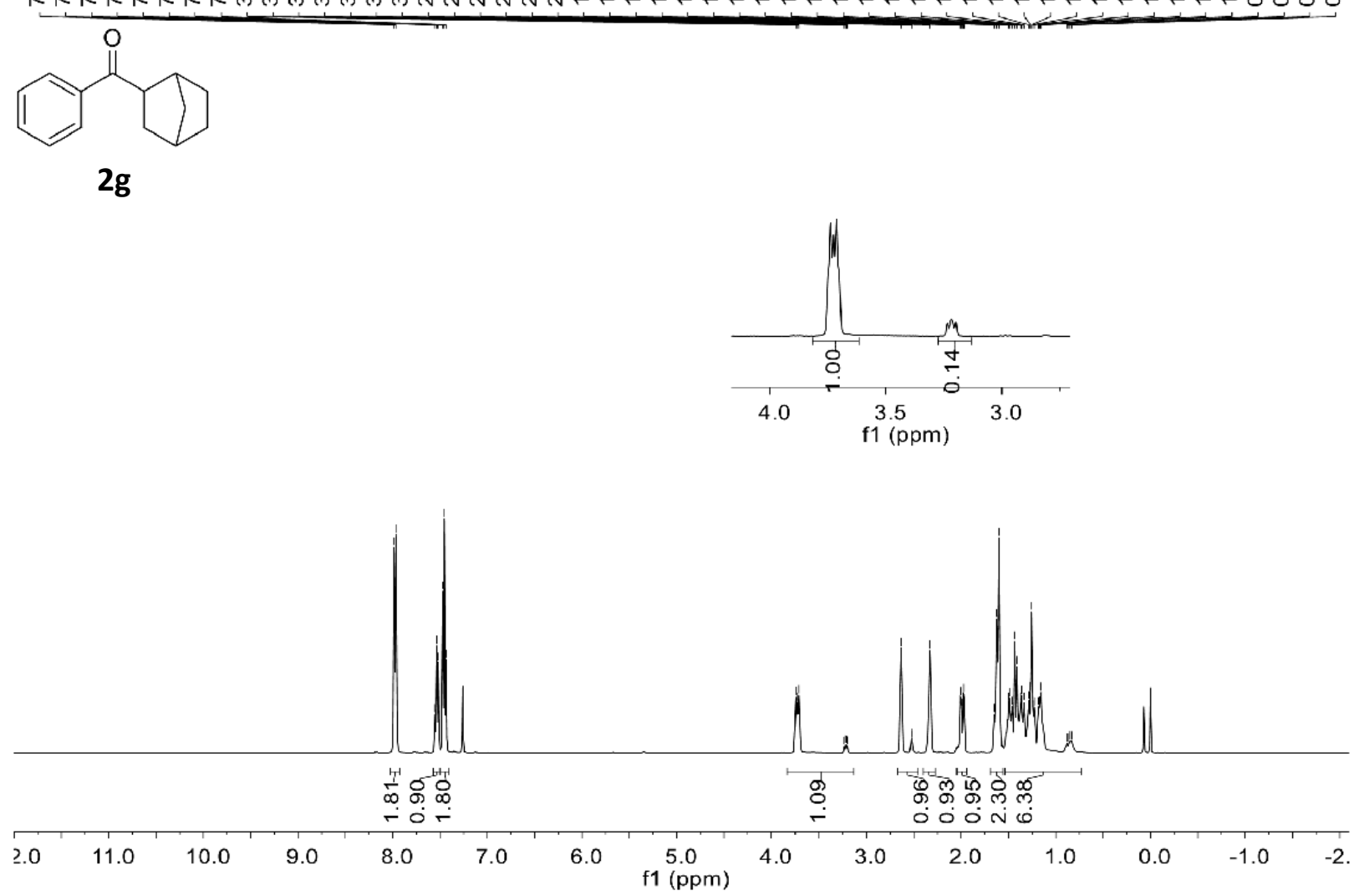

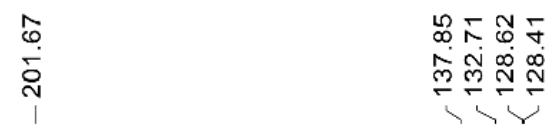

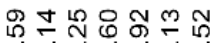

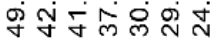

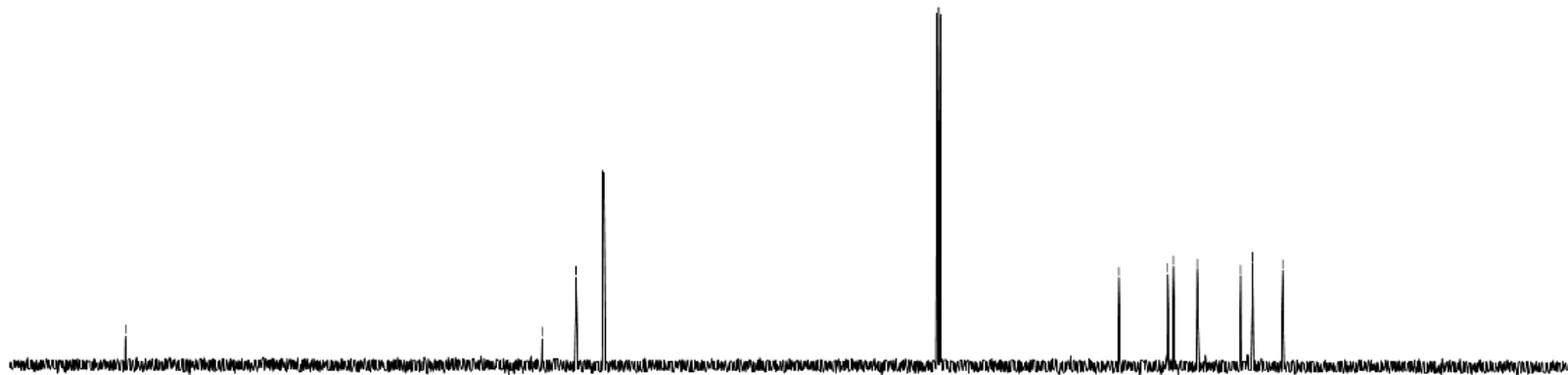

$\begin{array}{lllllllllllllllllllllll}210 & 200 & 190 & 180 & 170 & 160 & 150 & 140 & 130 & 120 & 110 \begin{array}{c}100 \\ \mathrm{f} 1(\mathrm{ppm})\end{array} & 90 & 80 & 70 & 60 & 50 & 40 & 30 & 20 & 10 & 0 & -10\end{array}$ 
ஹ ฌ

NNN

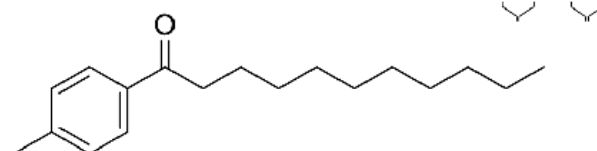

$2 \mathrm{~h}$

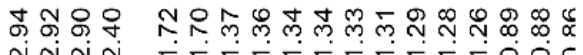

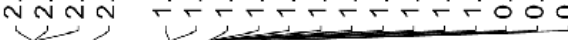

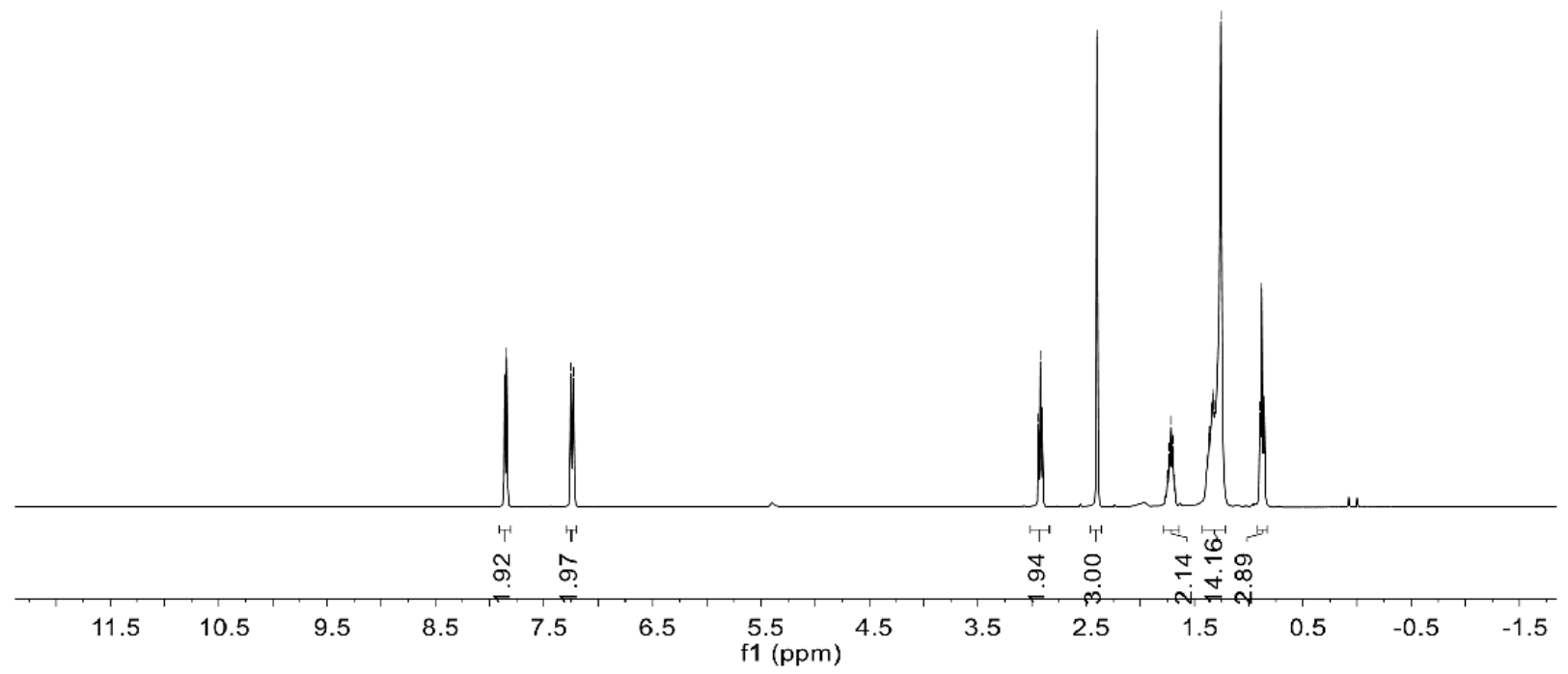

D্

ํํํ요 우

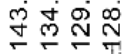

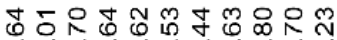

价 N

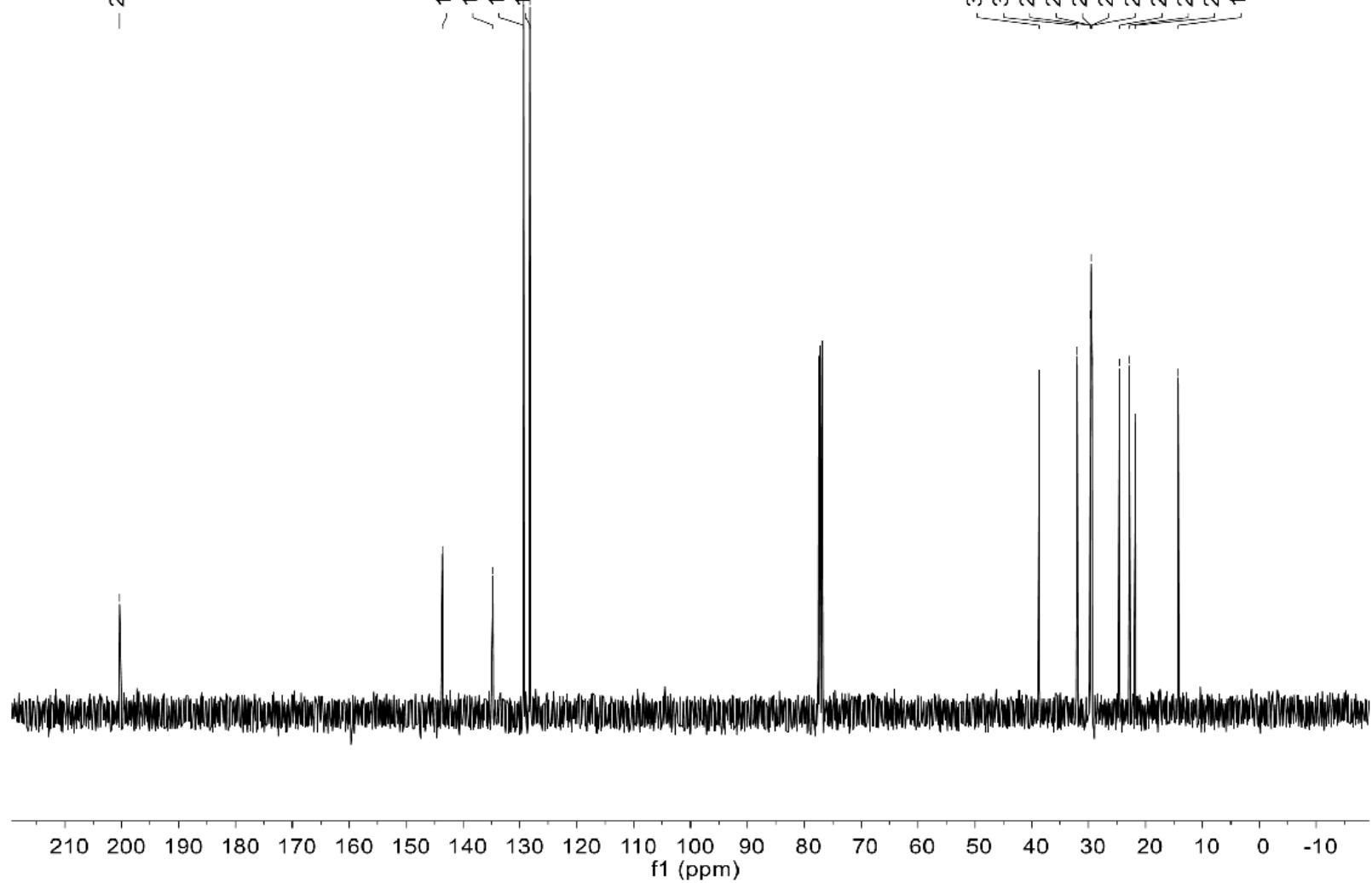




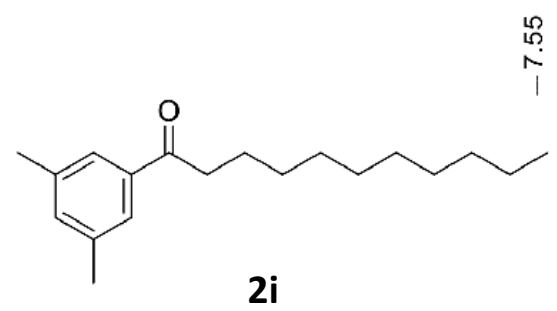

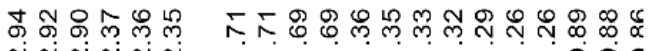

กั

กั่ กัก

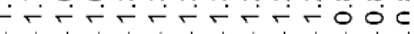

$2 \mathbf{i}$

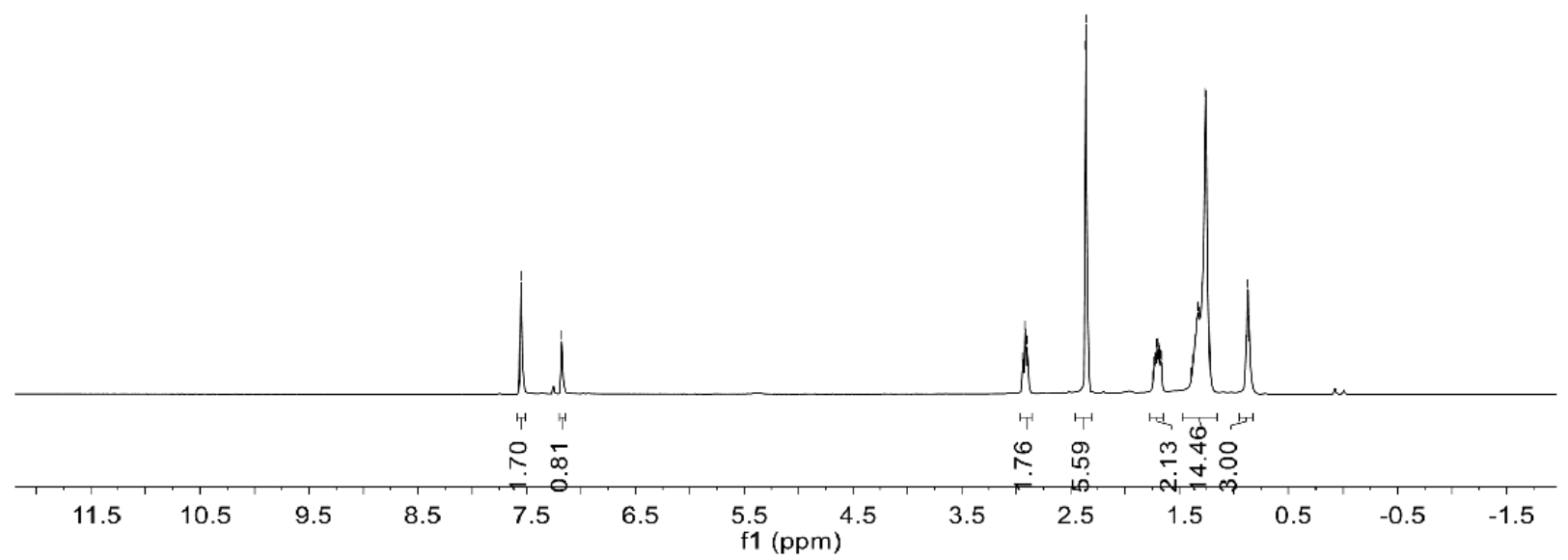

옹

ஸุ

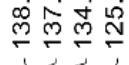

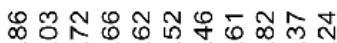

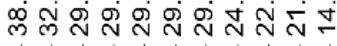

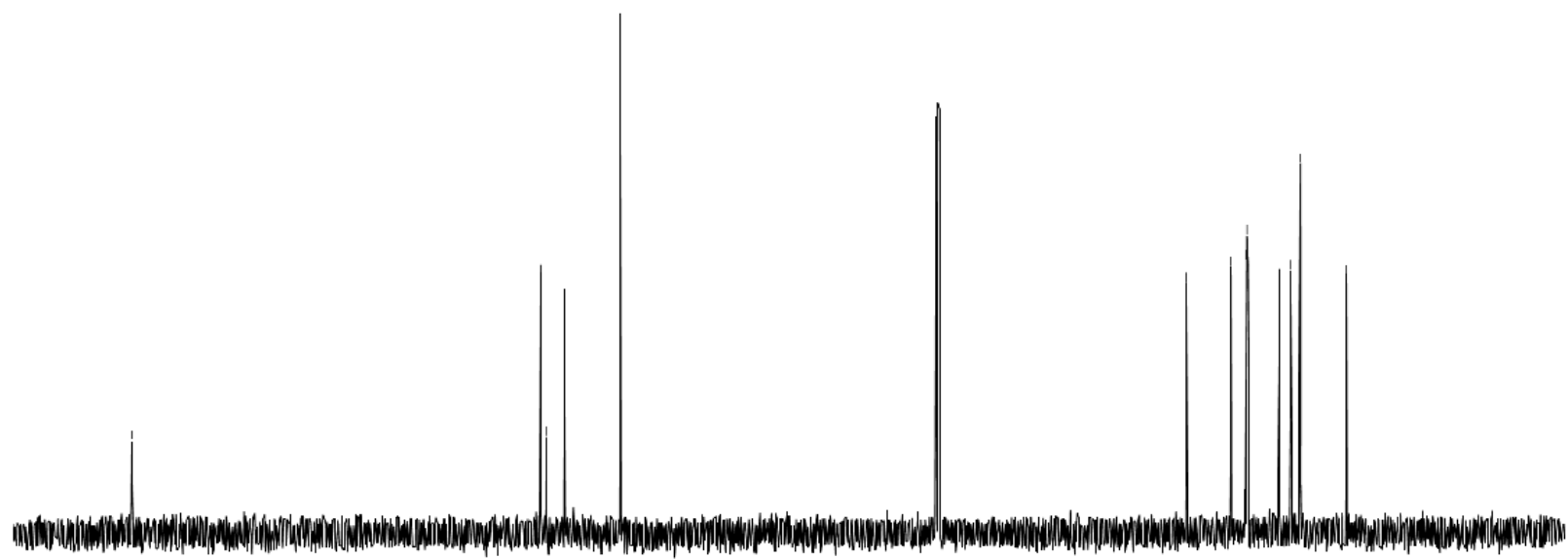

$\begin{array}{lllllllllllll}210 & 200 & 190 & 180 & 170 & 160 & 150 & 140 & 130 & 120 & 110 & 100 & 90\end{array}$

f1 (ppm) 

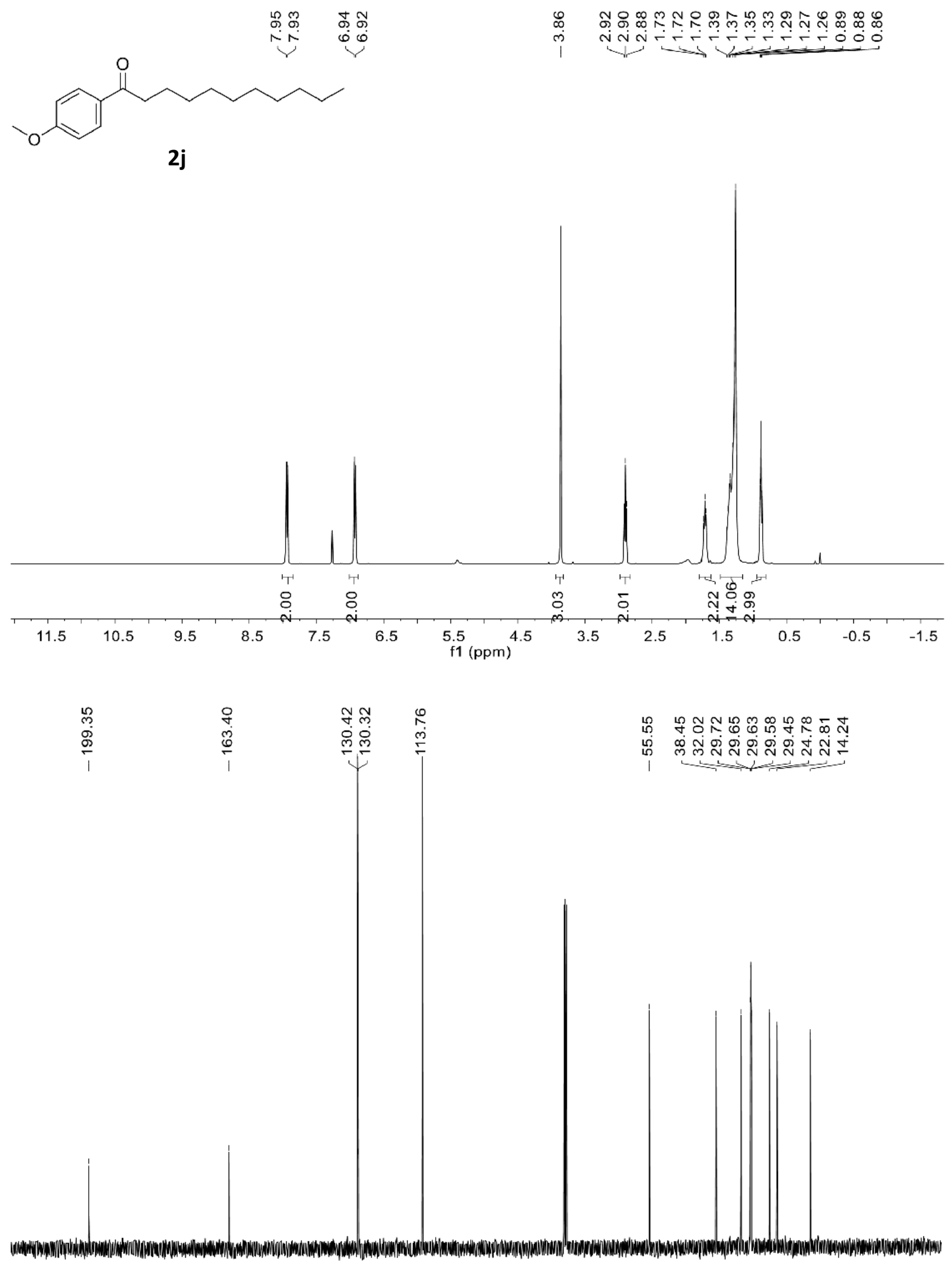

$\begin{array}{lllllllllllllllllllllll}210 & 200 & 190 & 180 & 170 & 160 & 150 & 140 & 130 & 120 & 110 & 100 & 90 & 80 & 70 & 60 & 50 & 40 & 30 & 20 & 10 & 0 & -10\end{array}$ f1 $(\mathrm{ppm})$ 

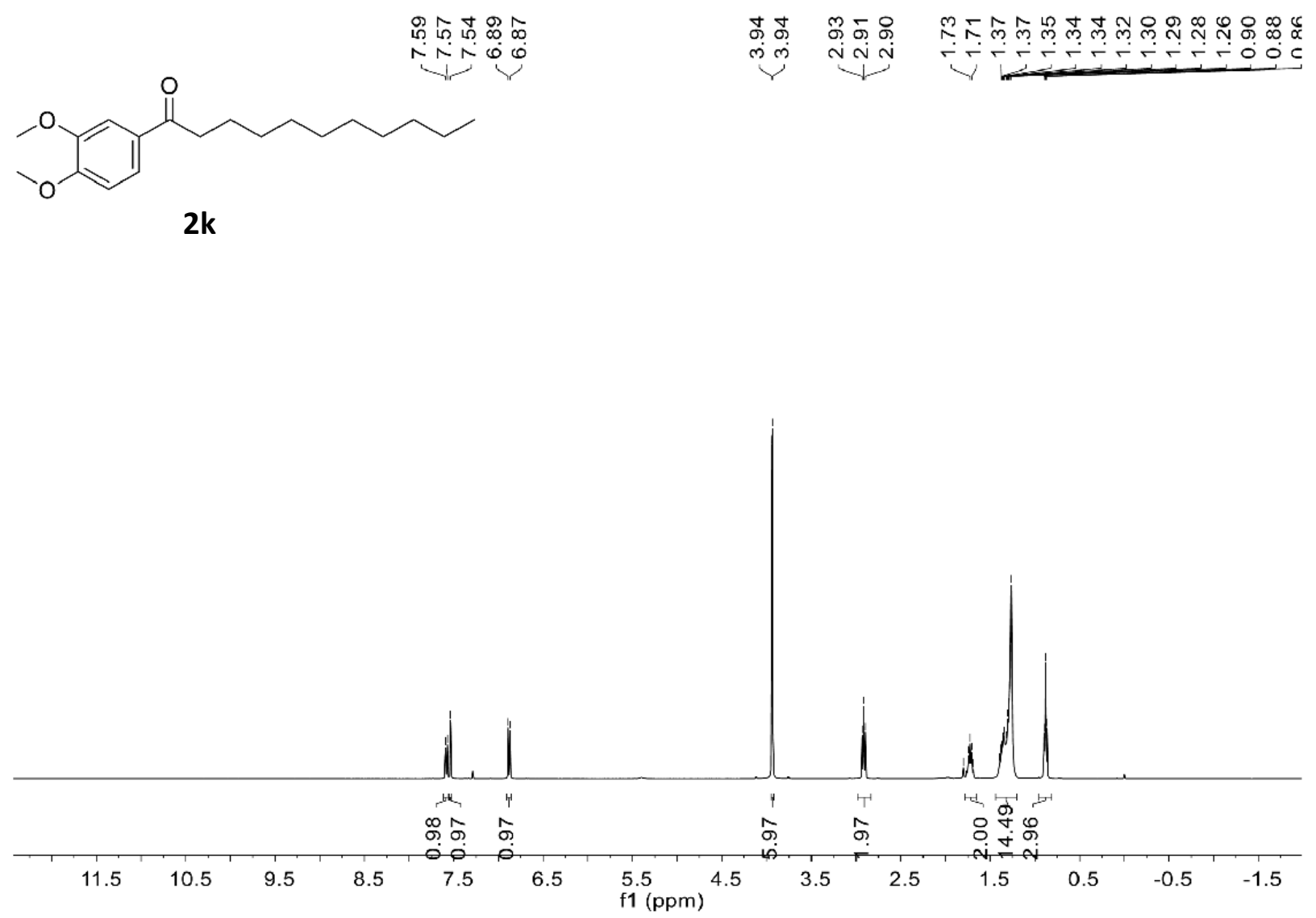

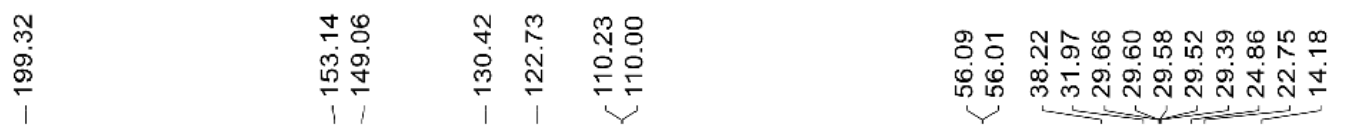

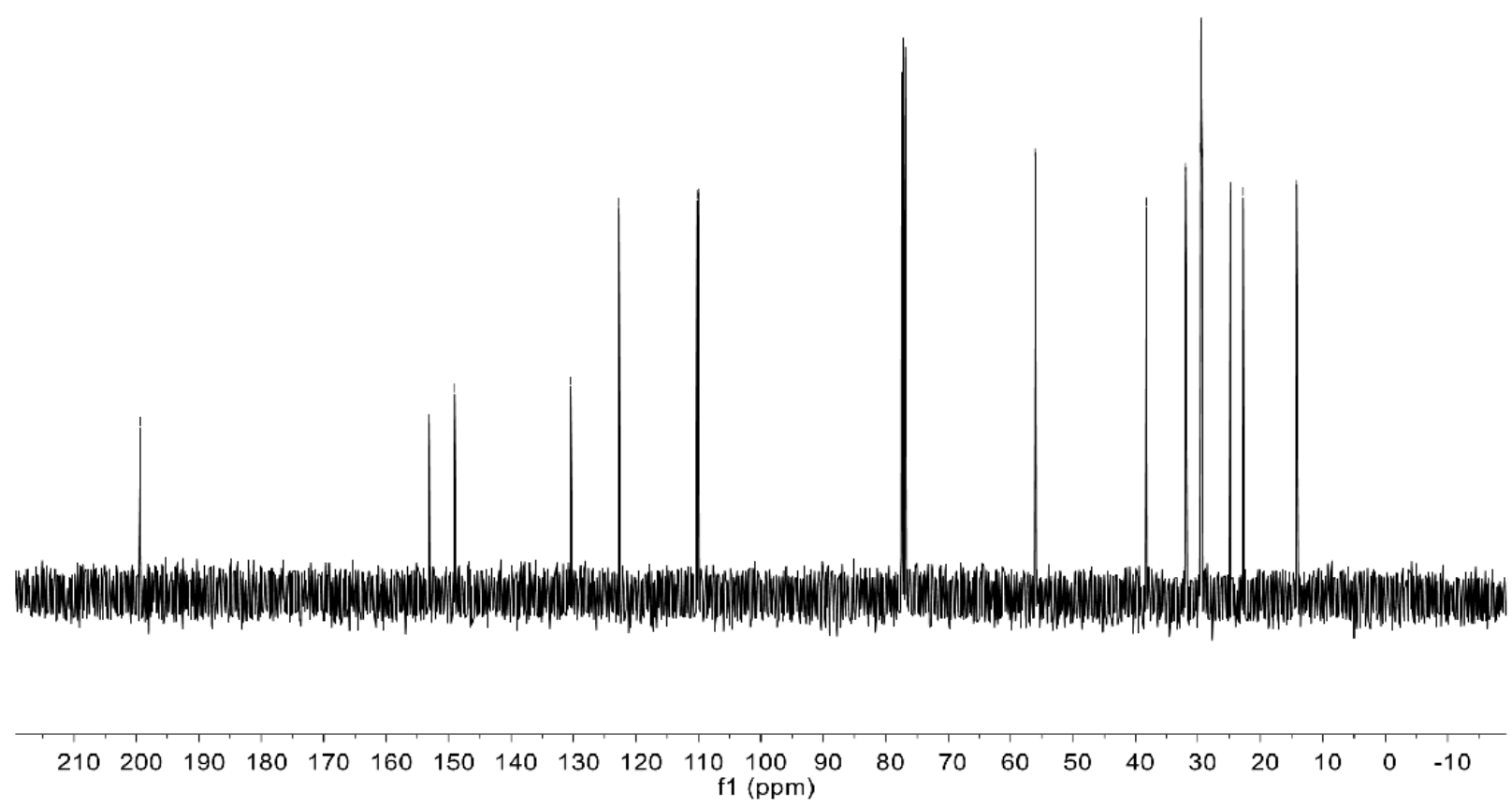




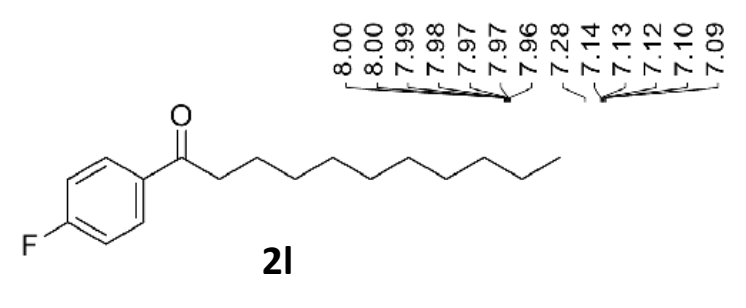

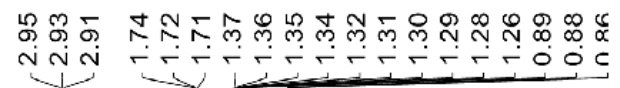

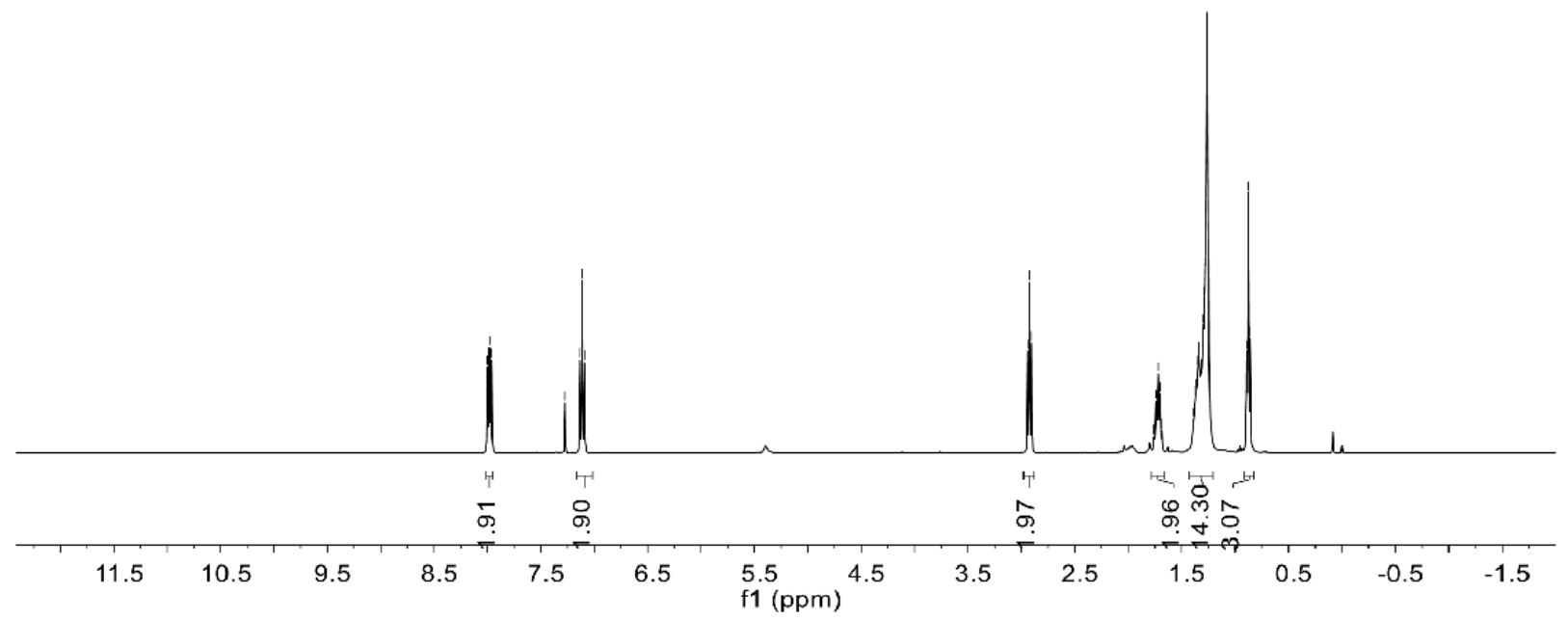

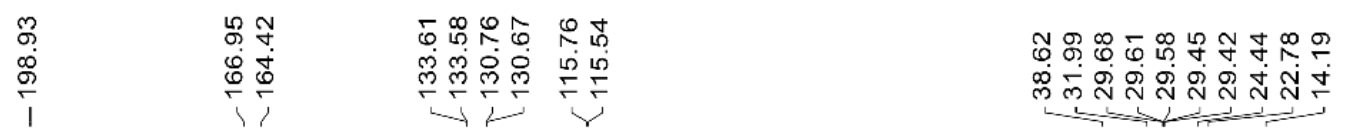

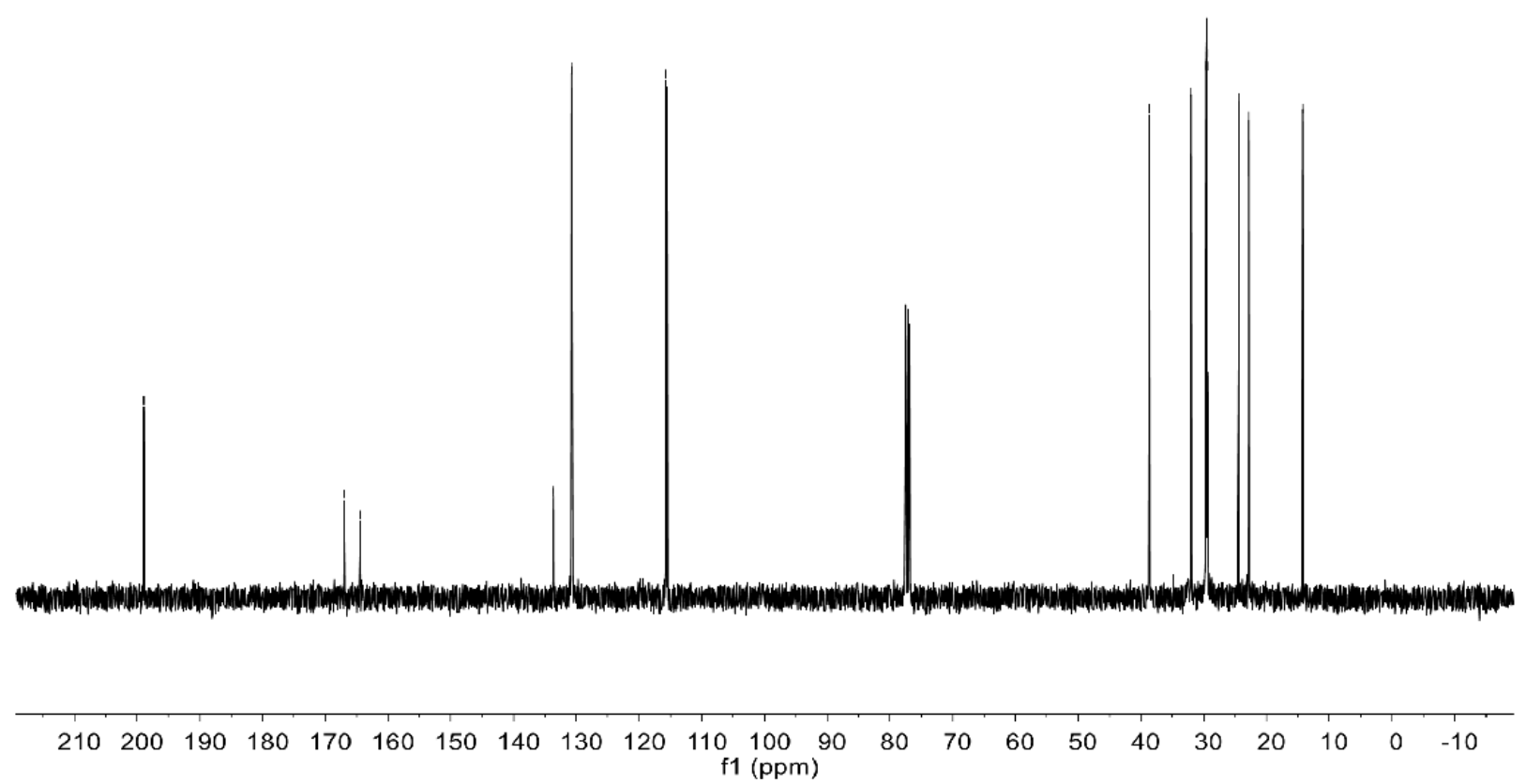




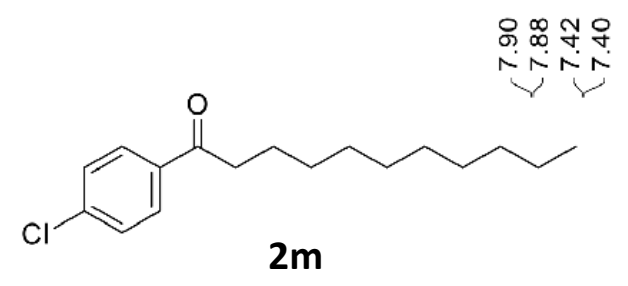

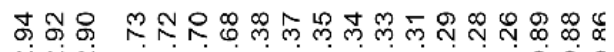

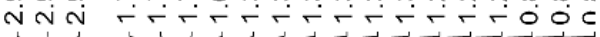
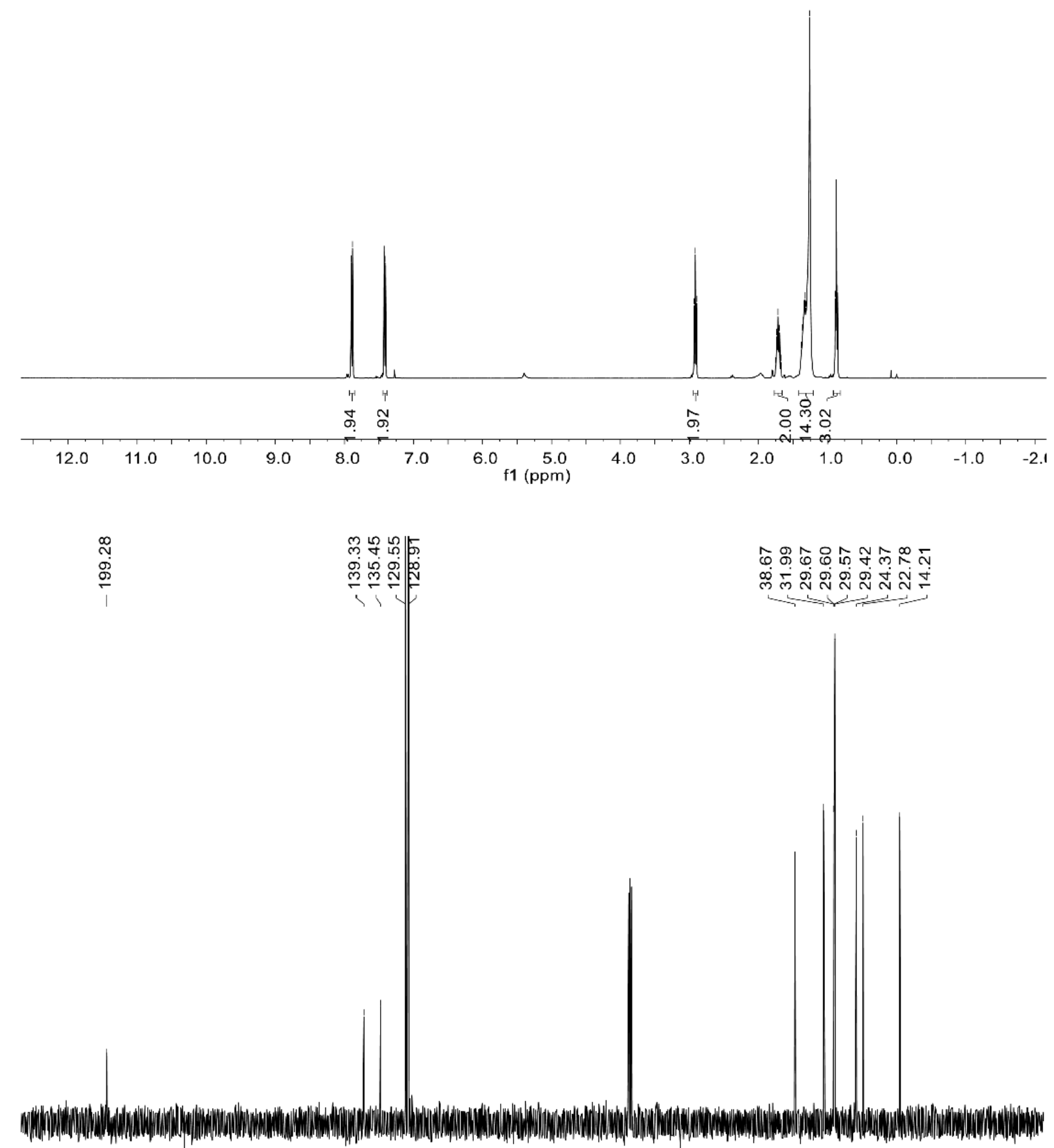

$\begin{array}{llllllllllllllllllllll}210 & 200 & 190 & 180 & 170 & 160 & 150 & 140 & 130 & 120 & \begin{array}{c}110 \\ \mathrm{f} 1\end{array}(\mathrm{ppm})\end{array}$ 


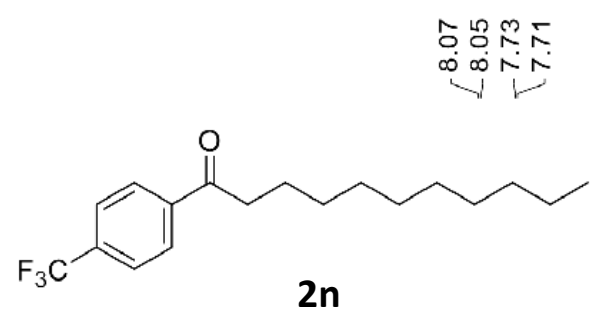

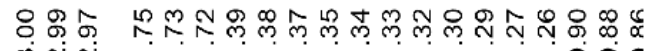

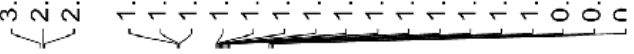

\section{$2 n$}
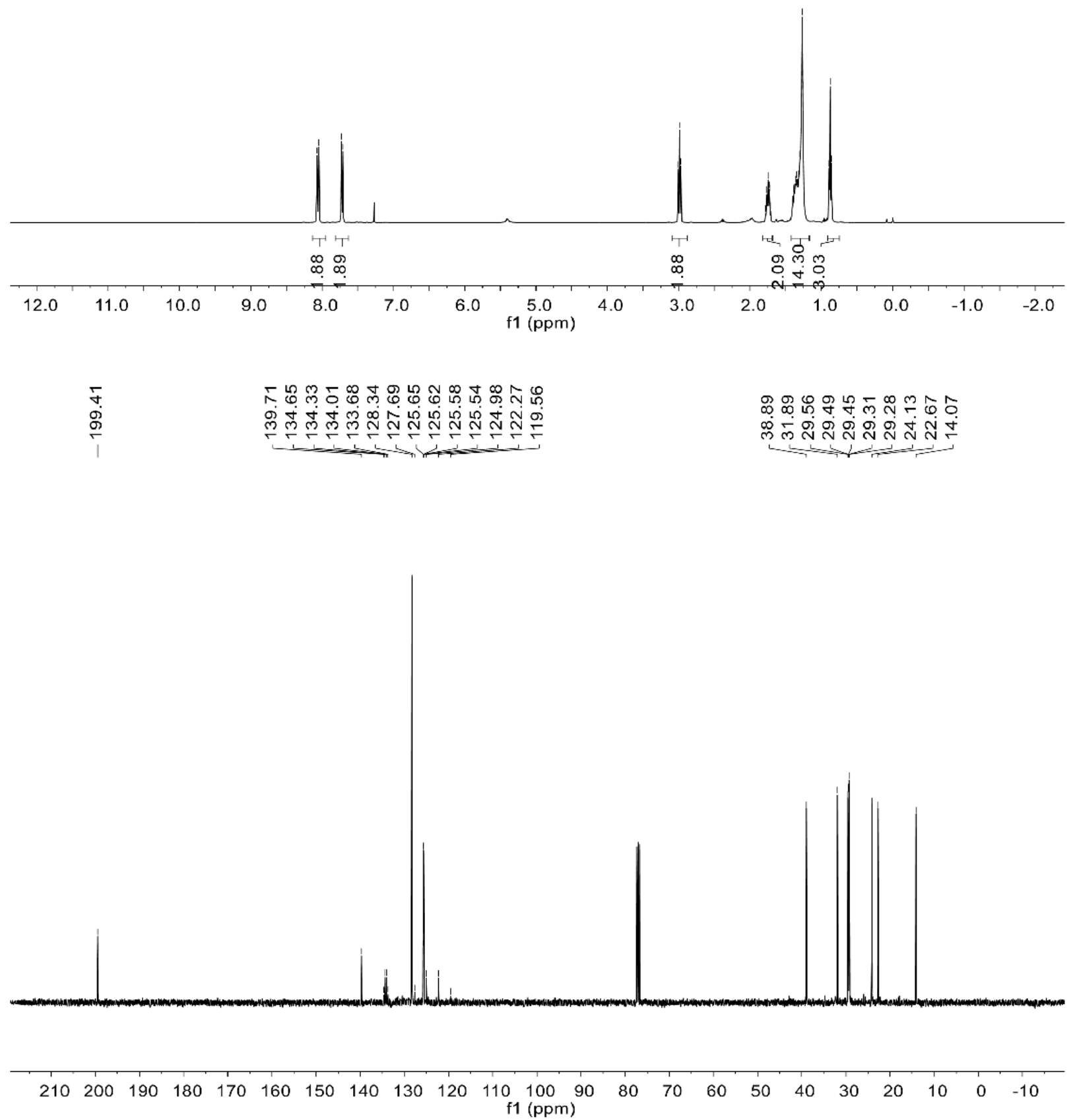


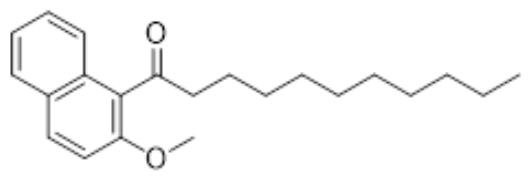

$2 p$

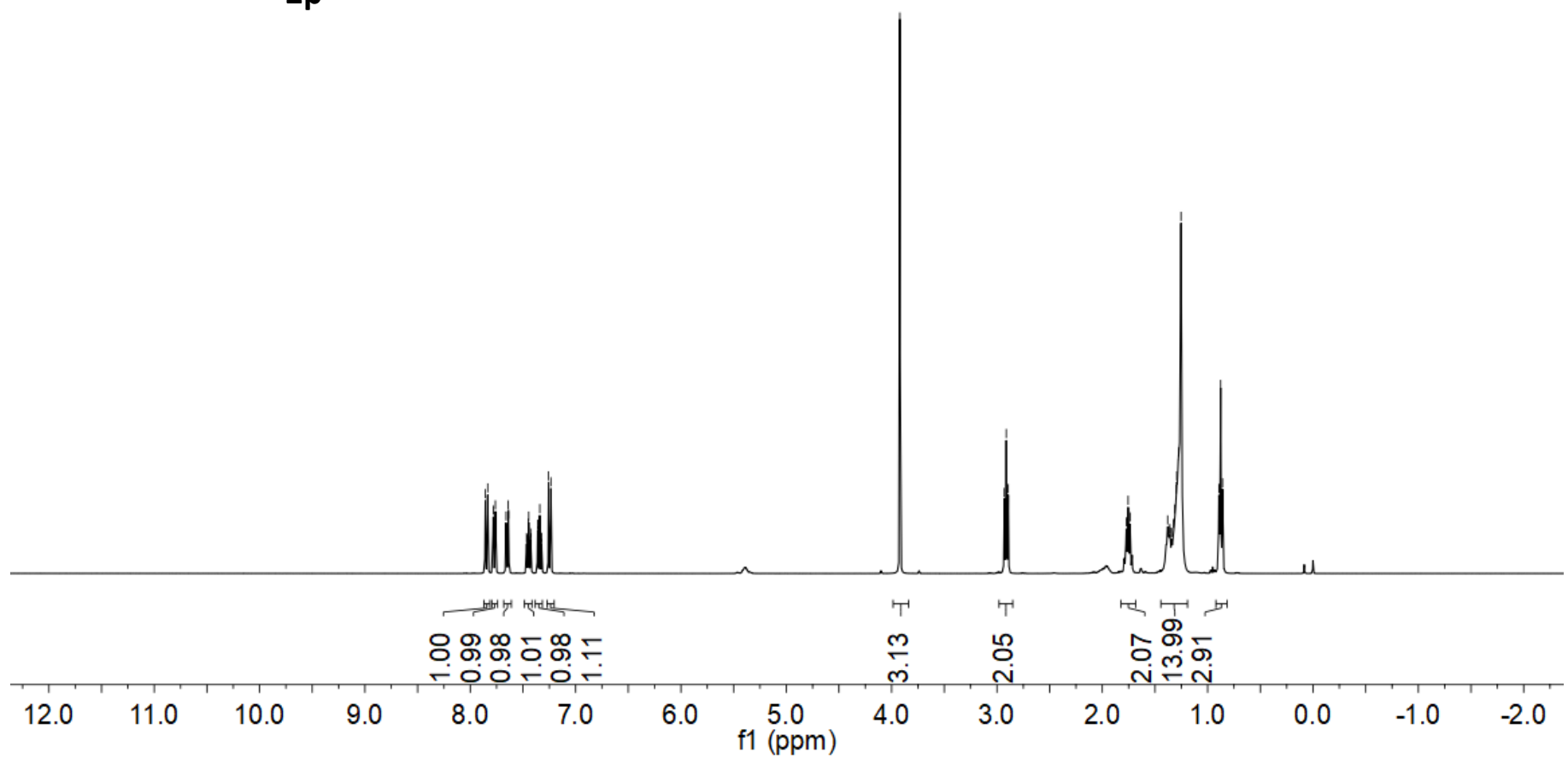

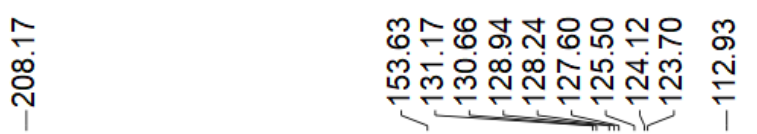

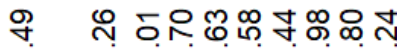

in

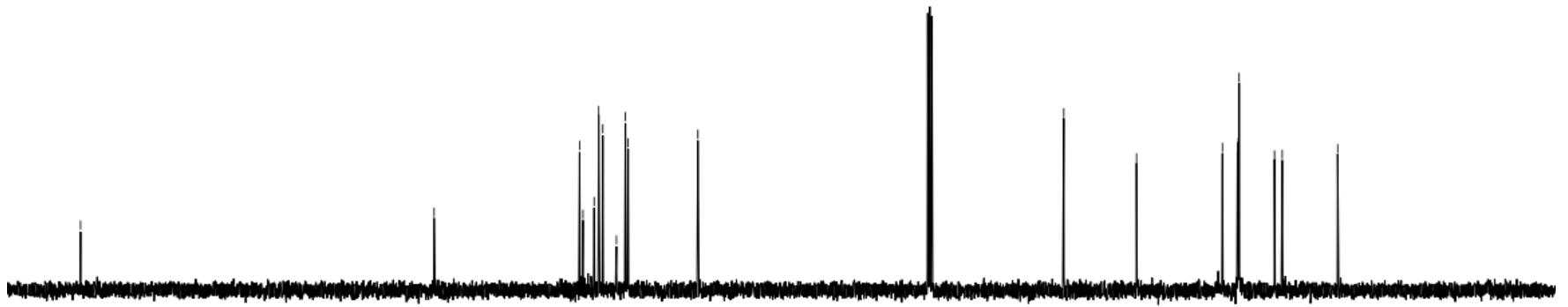

$\begin{array}{lllllllllllllllllllllll}210 & 200 & 190 & 180 & 170 & 160 & 150 & 140 & 130 & 120 & 110 & \underset{\mathrm{f} 1}{(\mathrm{ppm})} \mathbf{( 0 0} & 90 & 80 & 70 & 60 & 50 & 40 & 30 & 20 & 10 & 0 & -10\end{array}$ 
<smiles>CCCCC(=O)c1ccco1</smiles>

$2 q$

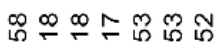

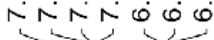

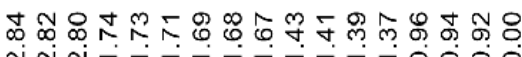

ninititictitiono

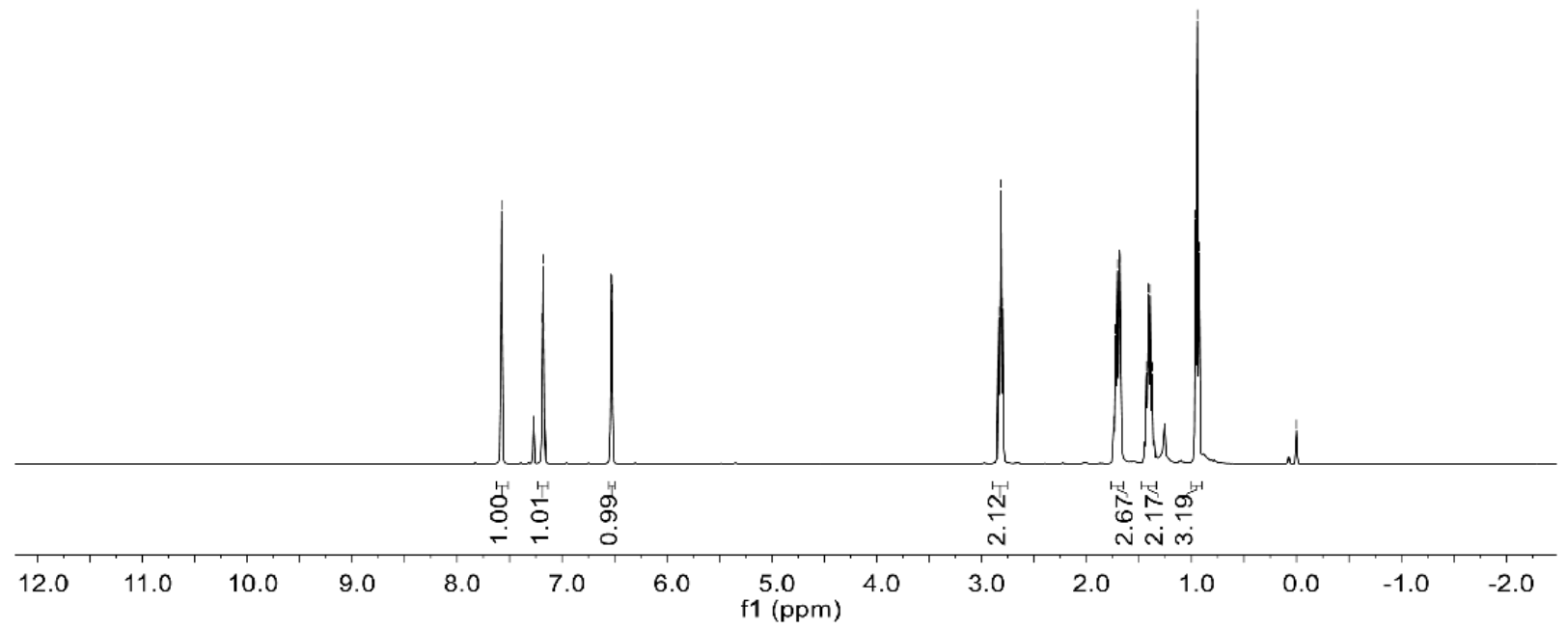

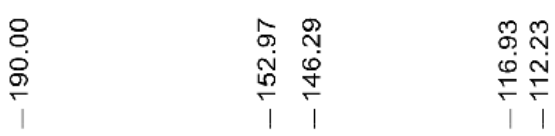

กิ กิ

品 뉸

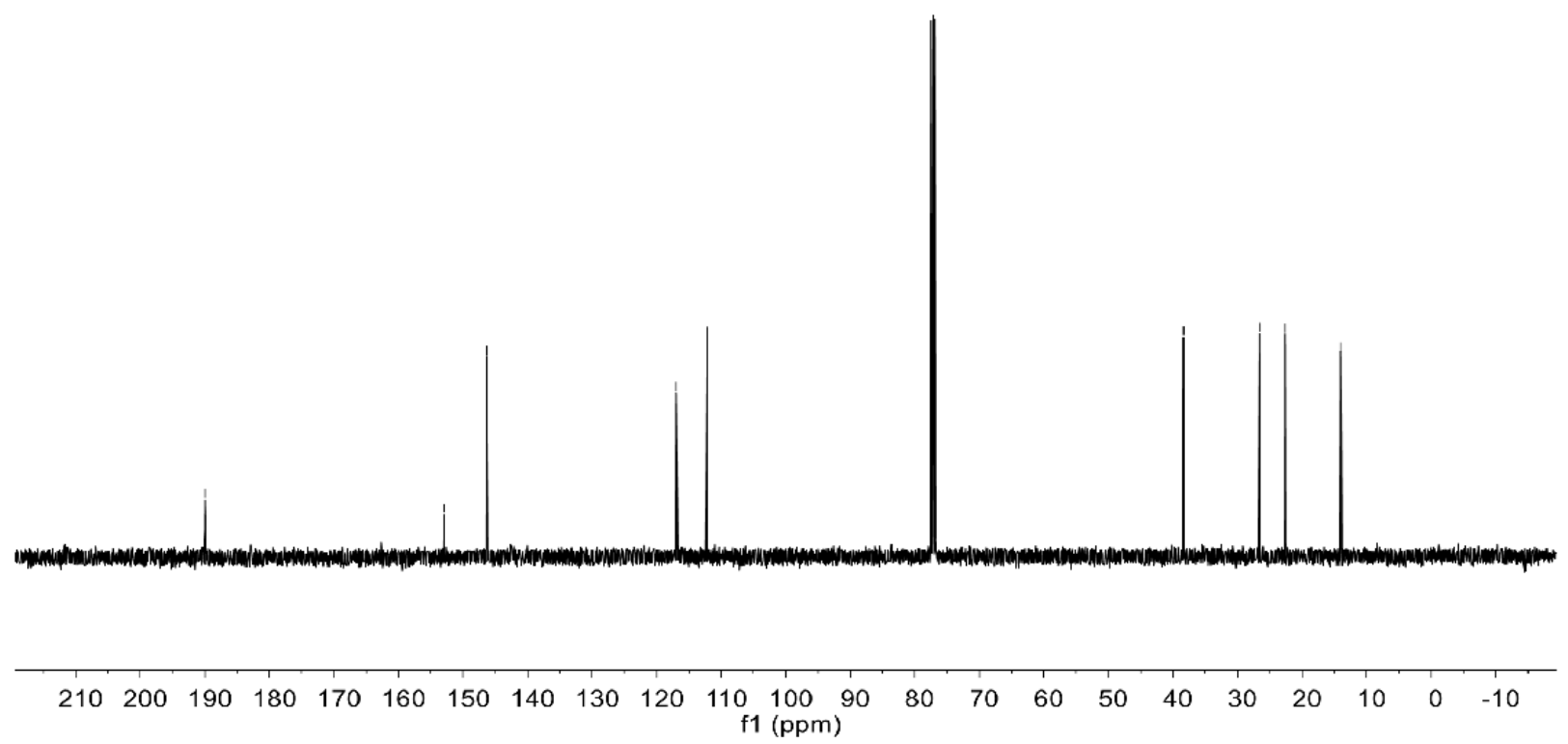




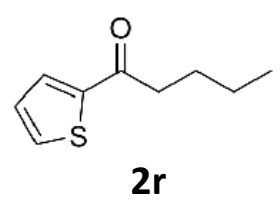

กัก

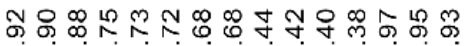

NNNNAN

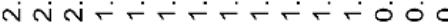

$2 r$

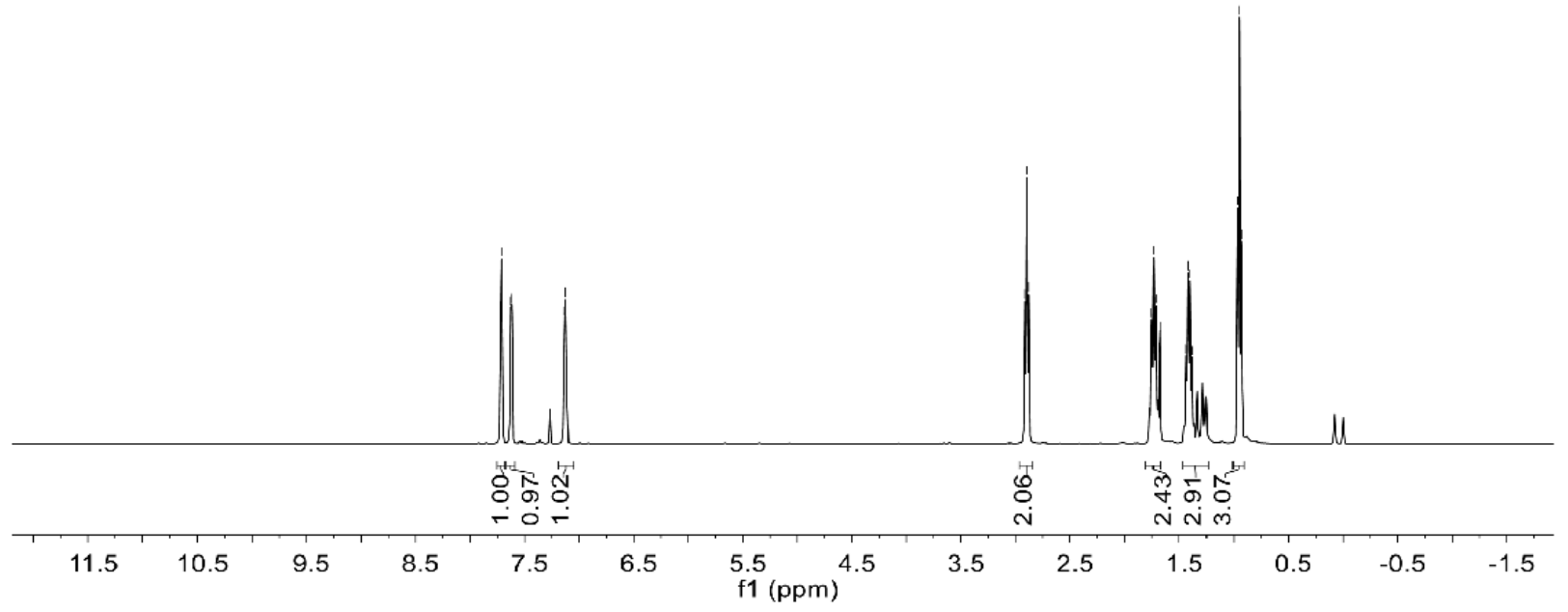

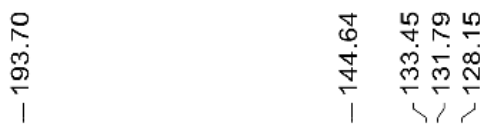

ล

อิ ลิกั้

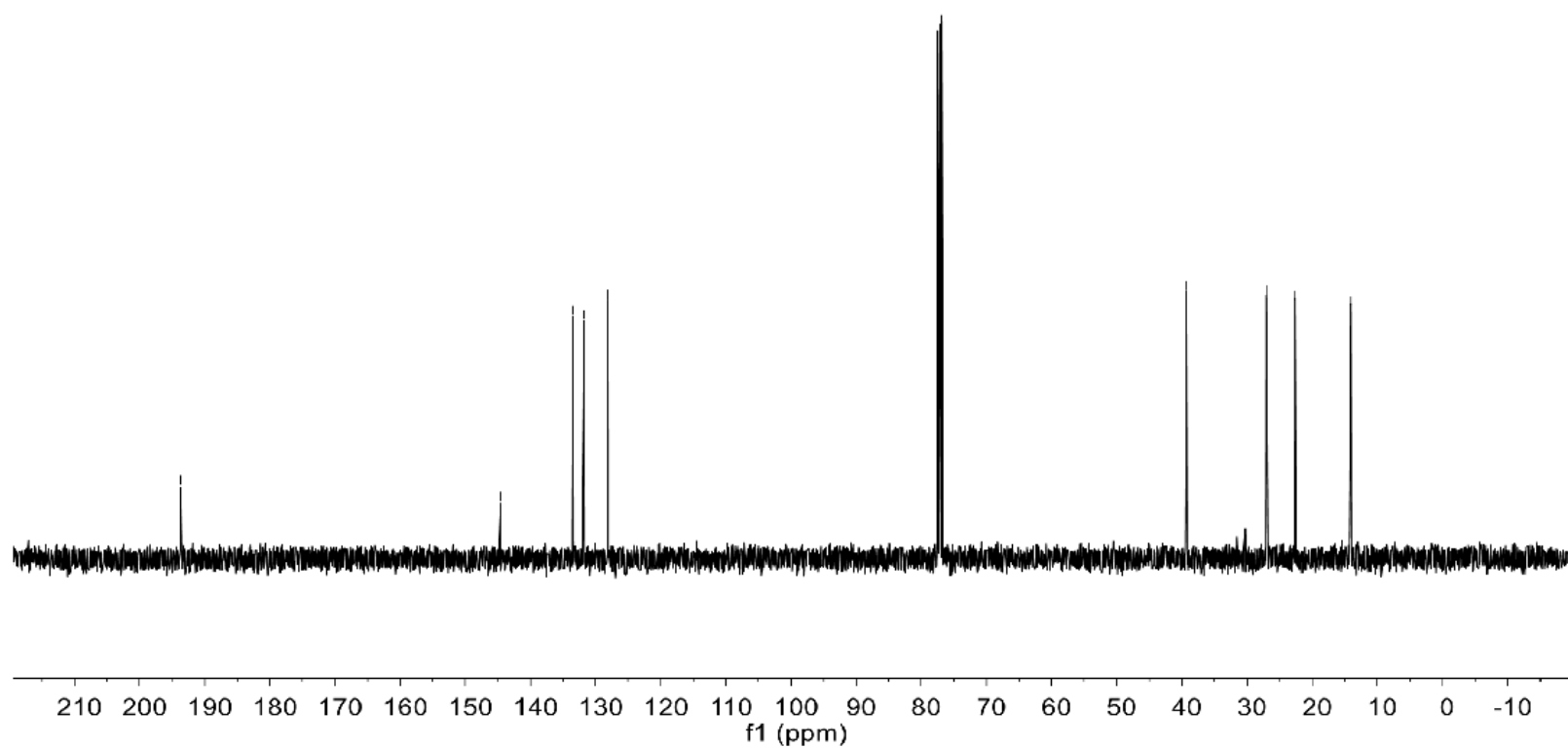



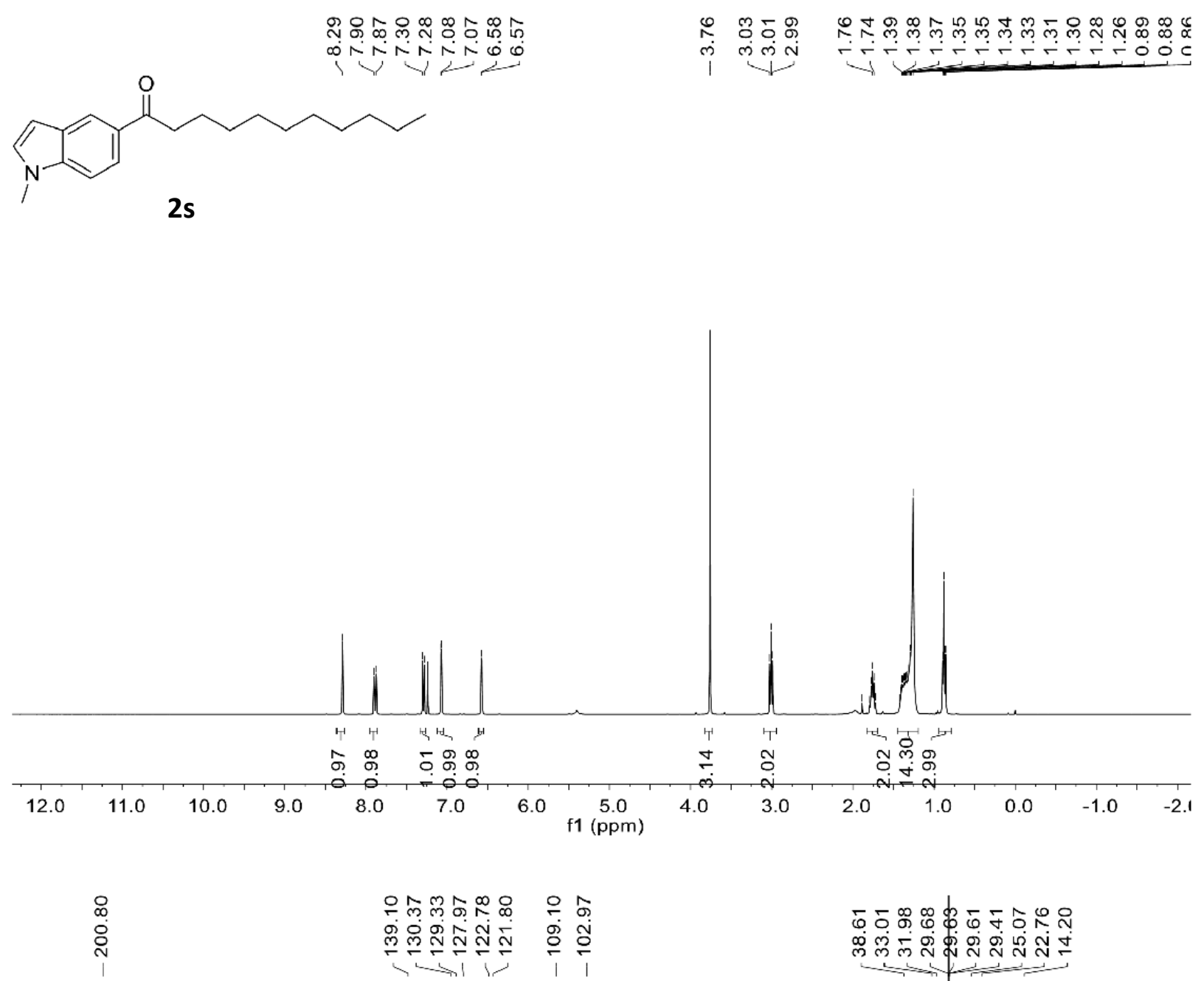

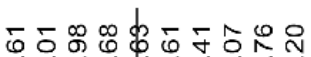

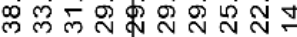

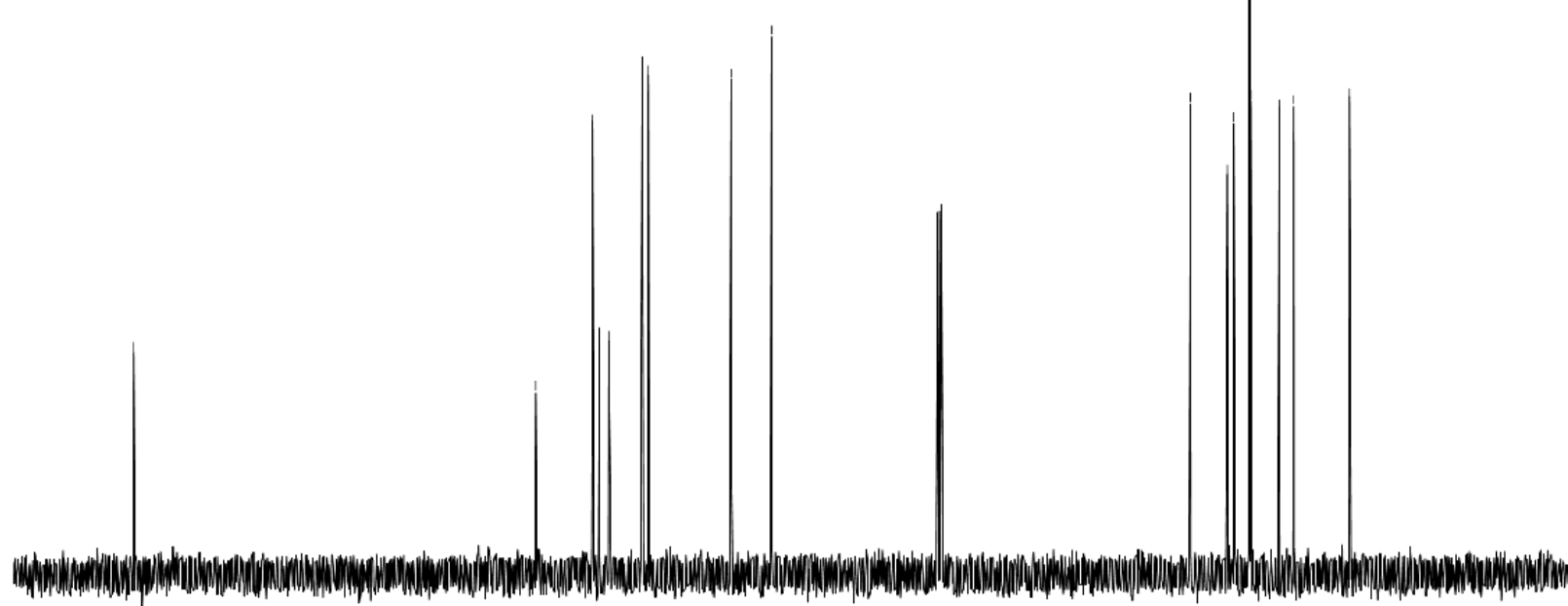

$\begin{array}{lllllllllllllllllllllll}210 & 200 & 190 & 180 & 170 & 160 & 150 & 140 & 130 & 120 & 110 & 100 & 90 & 80 & 70 & 60 & 50 & 40 & 30 & 20 & 10 & 0 & -10\end{array}$ 

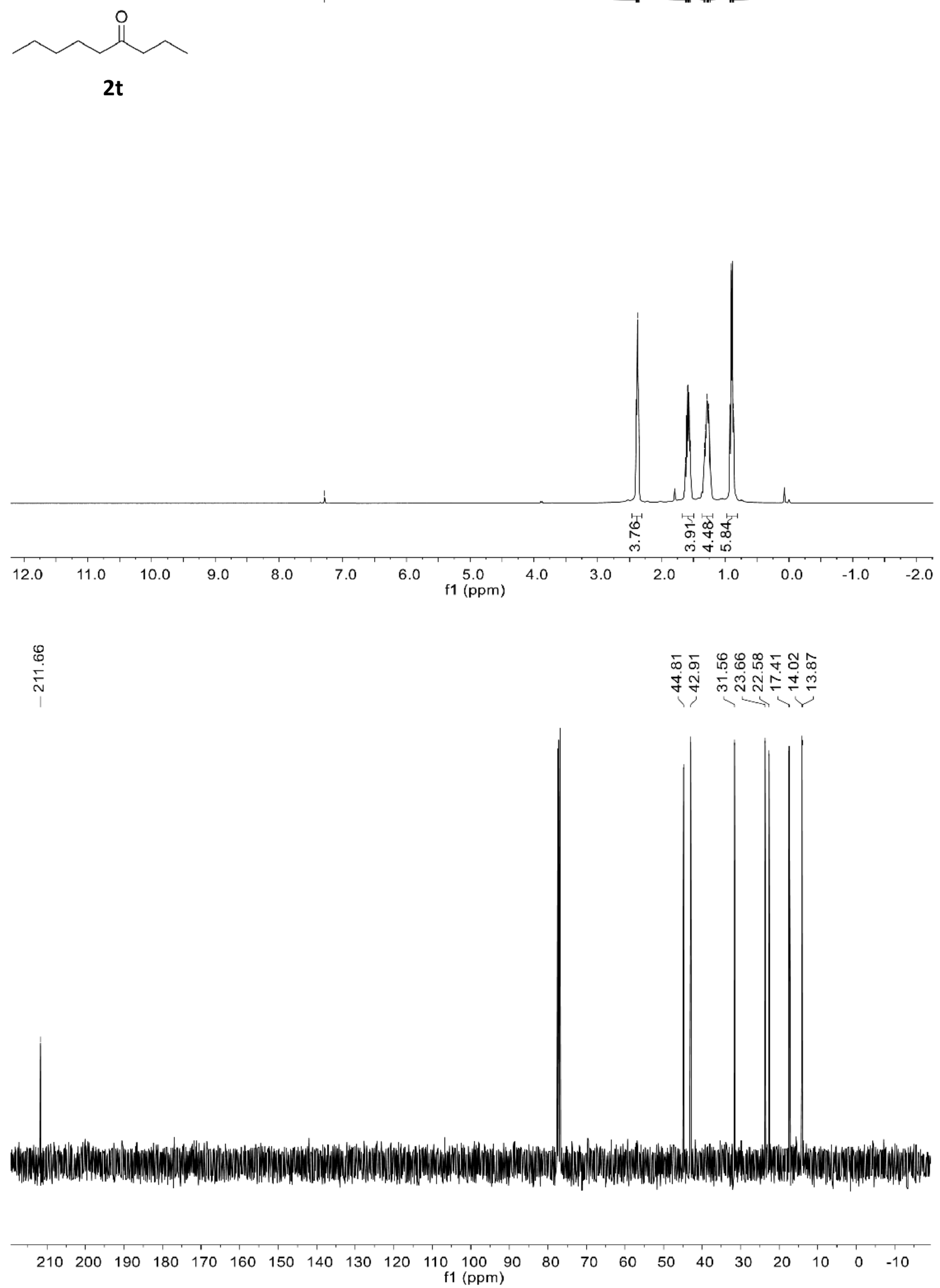
సุ
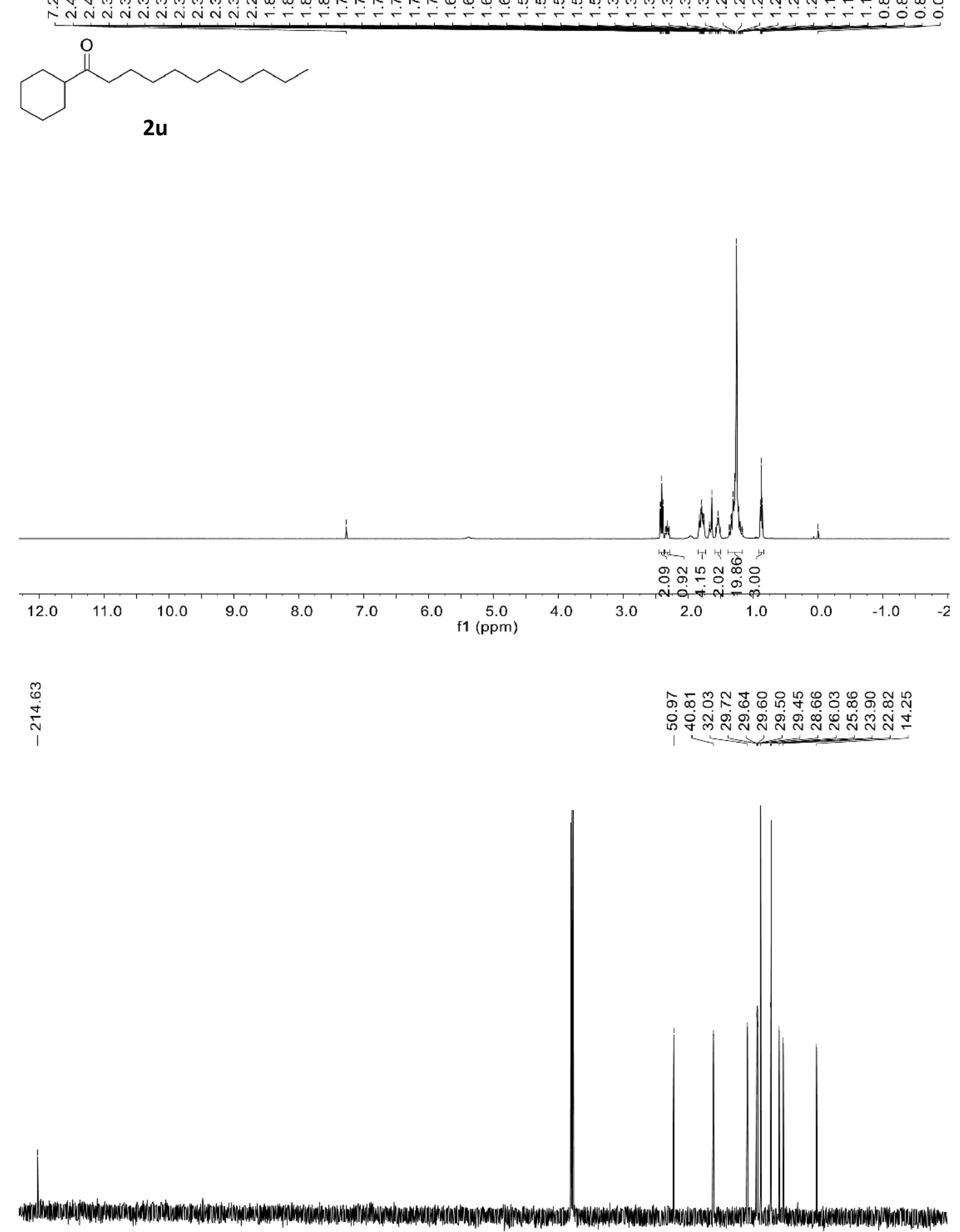

$\begin{array}{llllllllllllllllllllllll}210 & 200 & 190 & 180 & 170 & 160 & 150 & 140 & 130 & 120 & 110 & 100 & 90 & 80 & 70 & 60 & 50 & 40 & 30 & 20 & 10 & 0 & -10\end{array}$ f1 (ppm) 
<smiles>CCCCCCCCCCC(=O)C1CCCC1</smiles>

2v

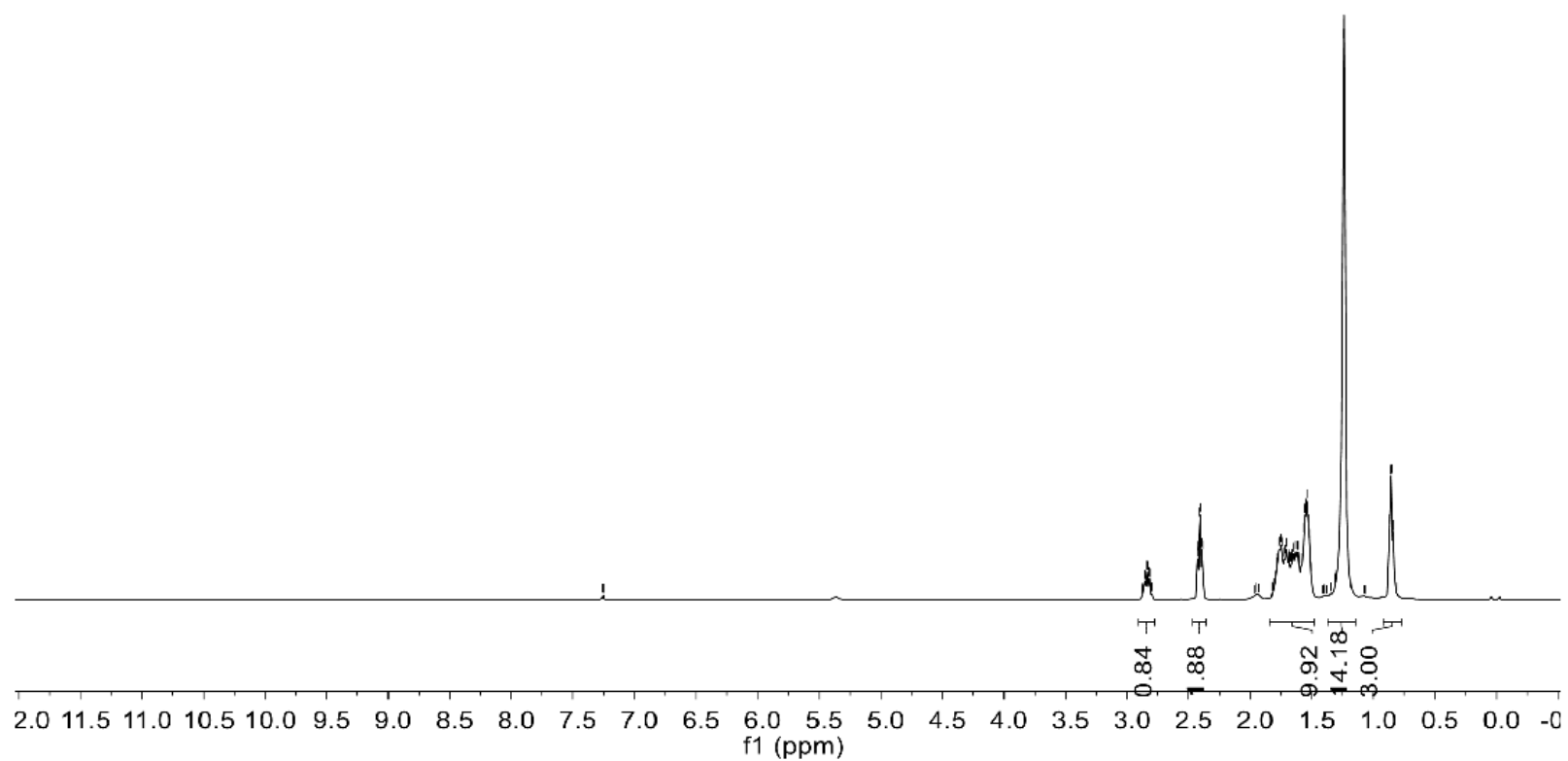

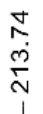

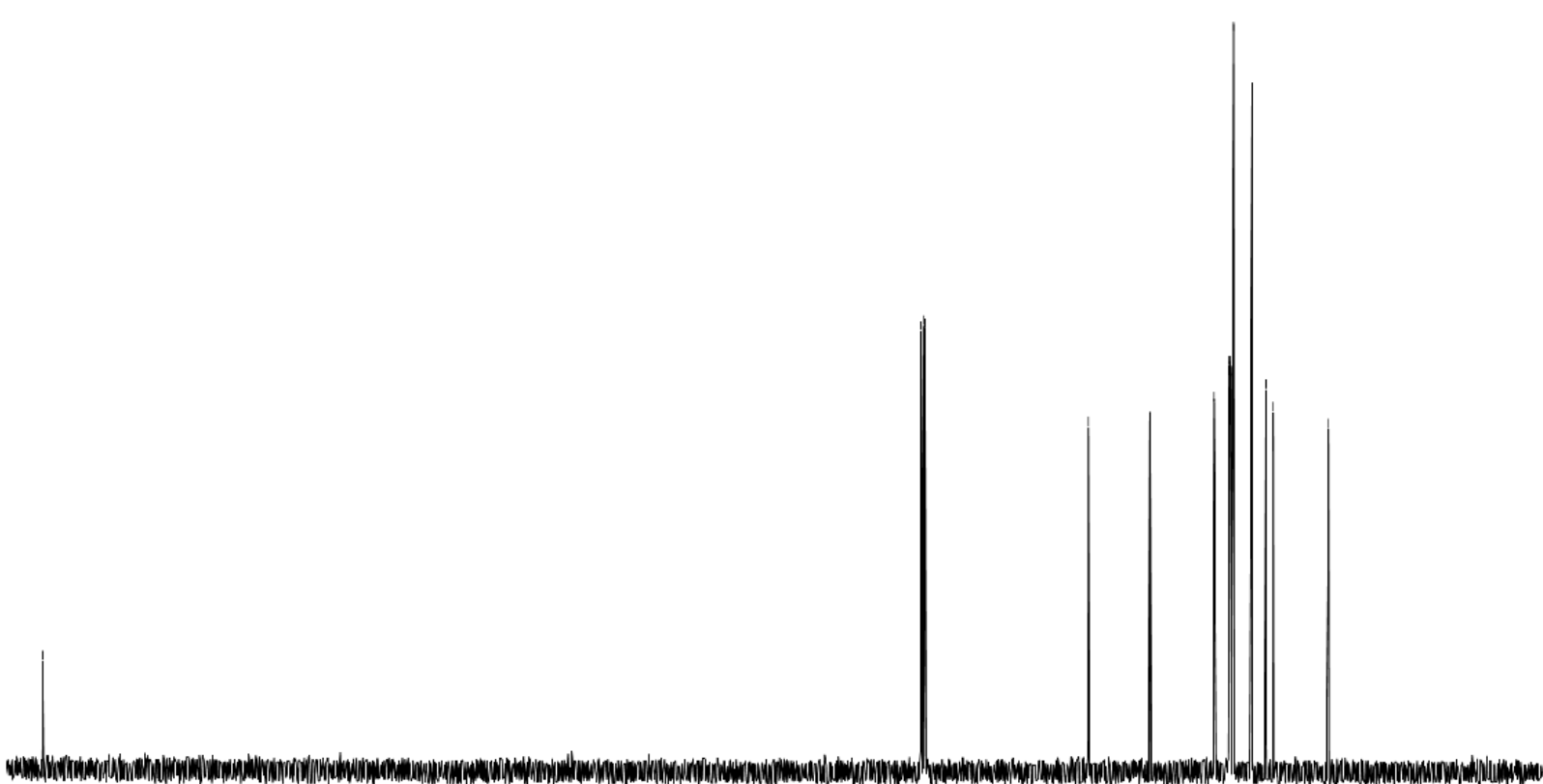

$\begin{array}{lllllllllllll}210 & 200 & 190 & 180 & 170 & 160 & 150 & 140 & 130 & 120 & 110 & 100 & 90\end{array}$

$\mathrm{f1}(\mathrm{ppm})$ 


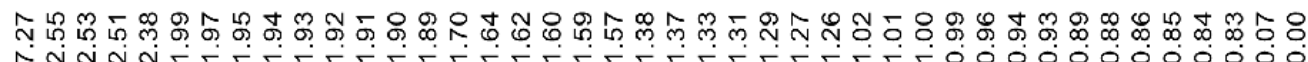

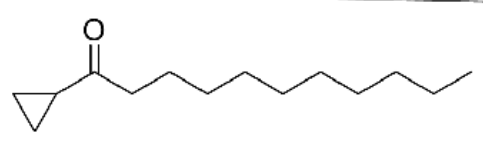

$2 w$

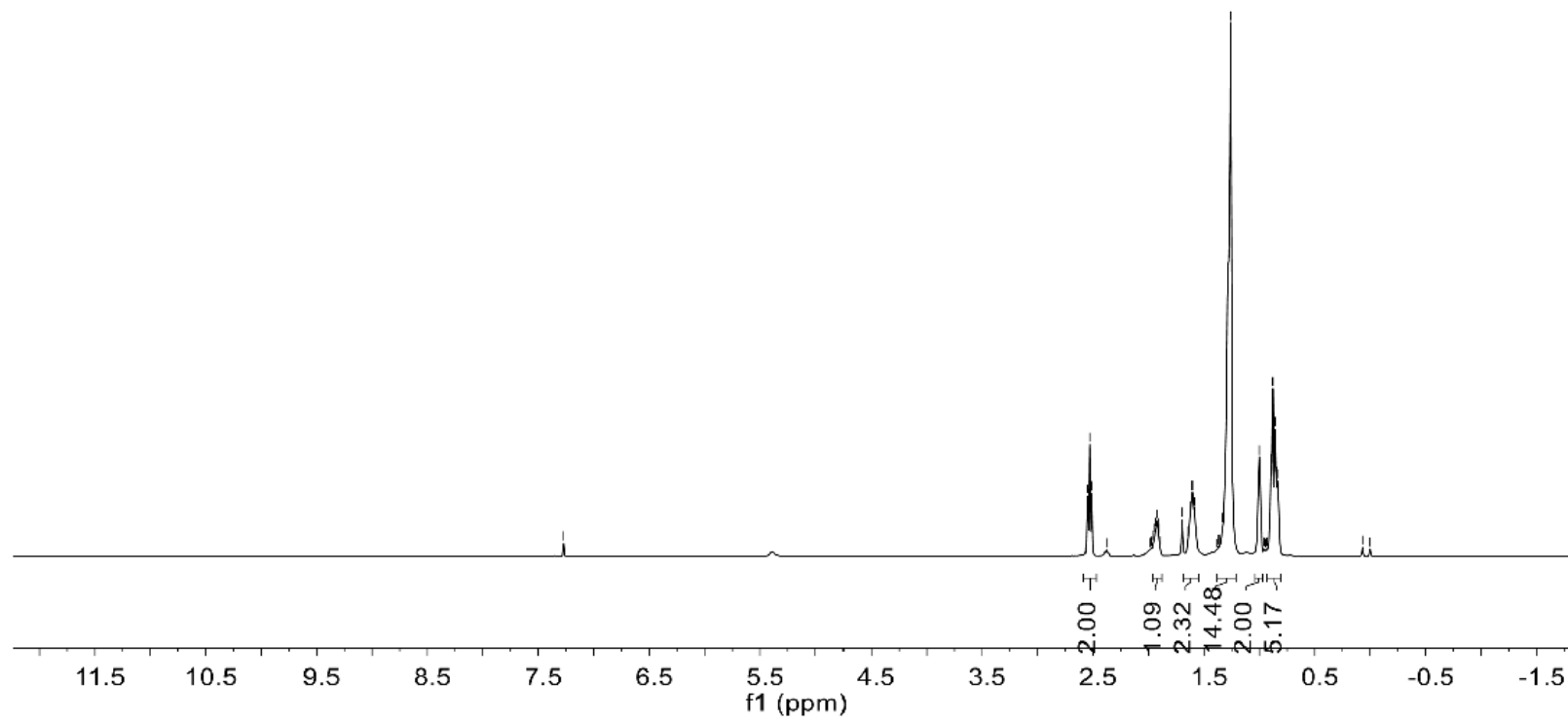

$\stackrel{\infty}{\stackrel{\infty}{\leftarrow}}$

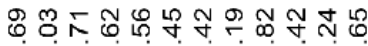

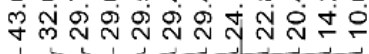

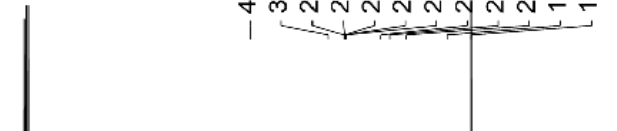

(N) w w w w

$\begin{array}{lllllllllllllllllllllll}210 & 200 & 190 & 180 & 170 & 160 & 150 & 140 & 130 & 120 & \begin{array}{c}110 \\ \mathrm{f} 1(\mathrm{ppm})\end{array}\left(\begin{array}{ll}100 \\ (\mathrm{pm})\end{array}\right. & 80 & 70 & 60 & 50 & 40 & 30 & 20 & 10 & 0 & -10\end{array}$ 


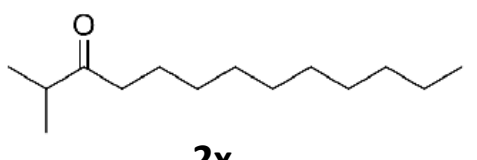

$2 x$

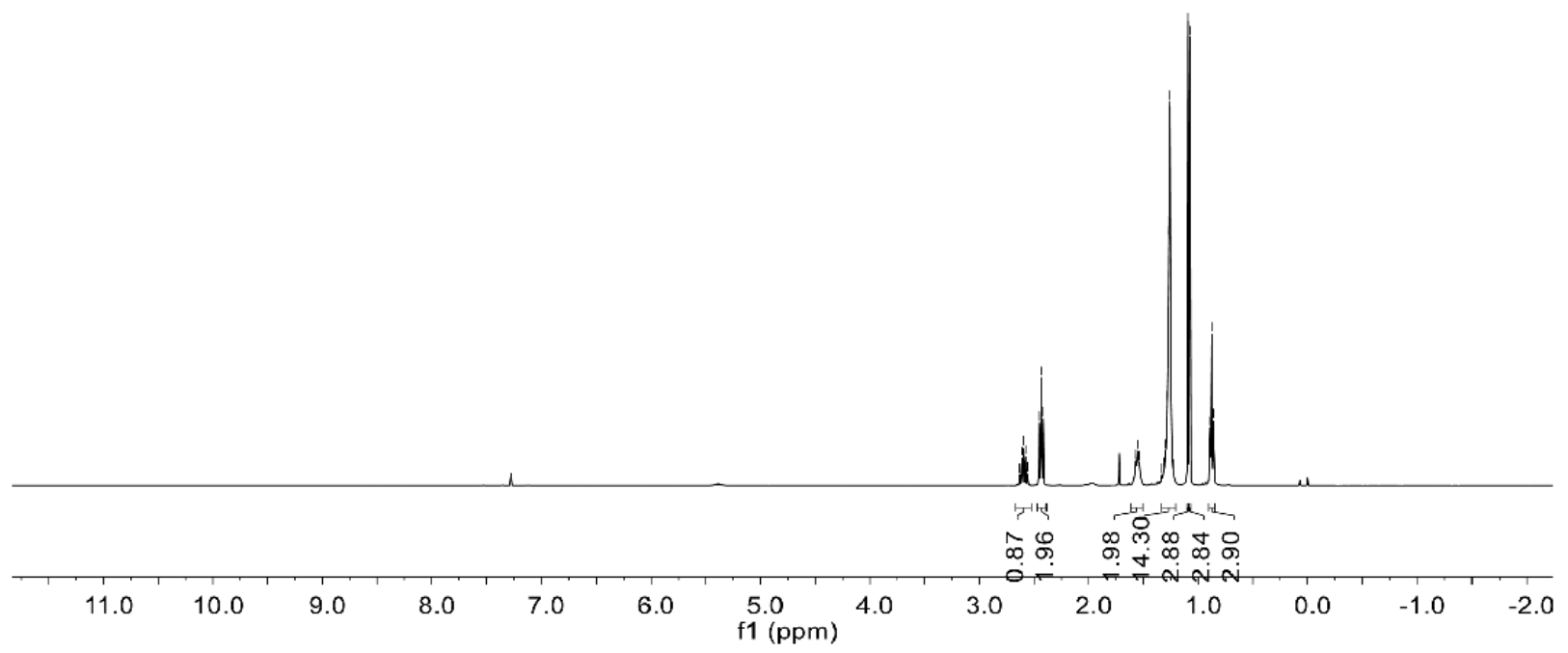

$\frac{\infty}{\frac{n}{n}}$

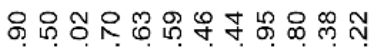
ชั่

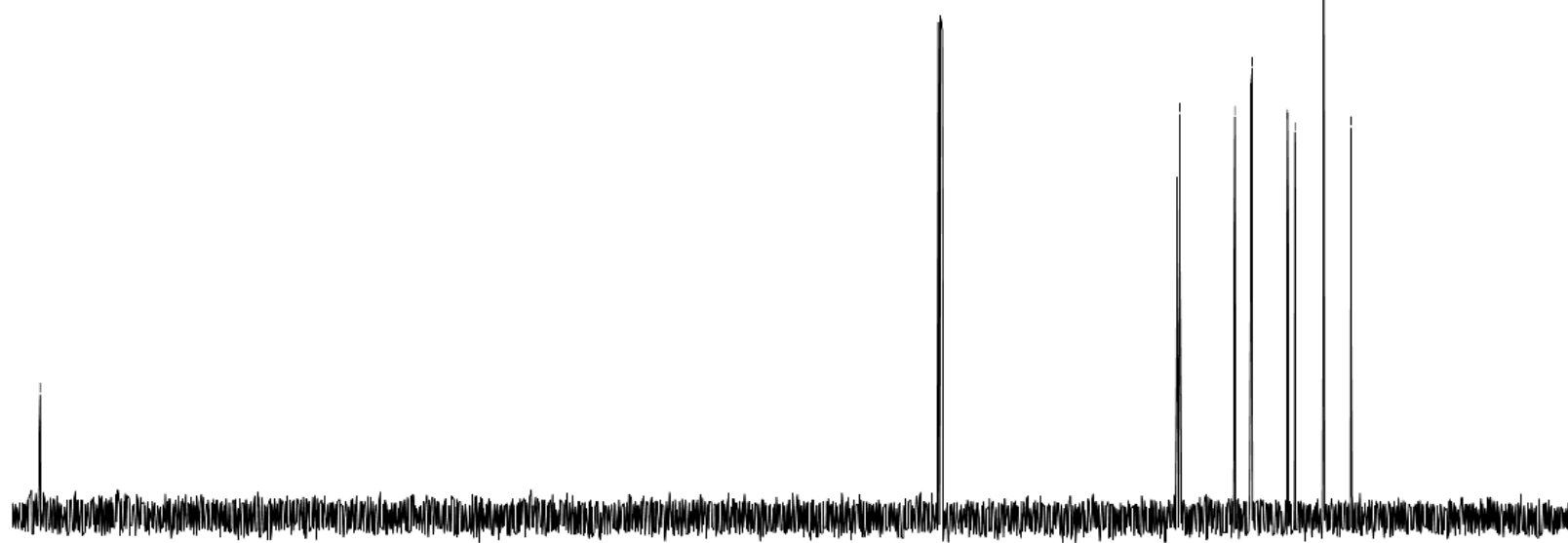

$\begin{array}{lllllllllllllllllllllll}210 & 200 & 190 & 180 & 170 & 160 & 150 & 140 & 130 & 120 & 110 \quad \begin{array}{c}100 \\ \mathrm{f} 1(\mathrm{ppm})\end{array} & 90 & 80 & 70 & 60 & 50 & 40 & 30 & 20 & 10 & 0 & -10\end{array}$ 


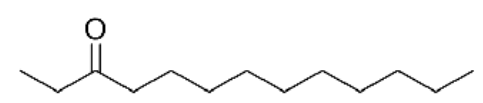

2y
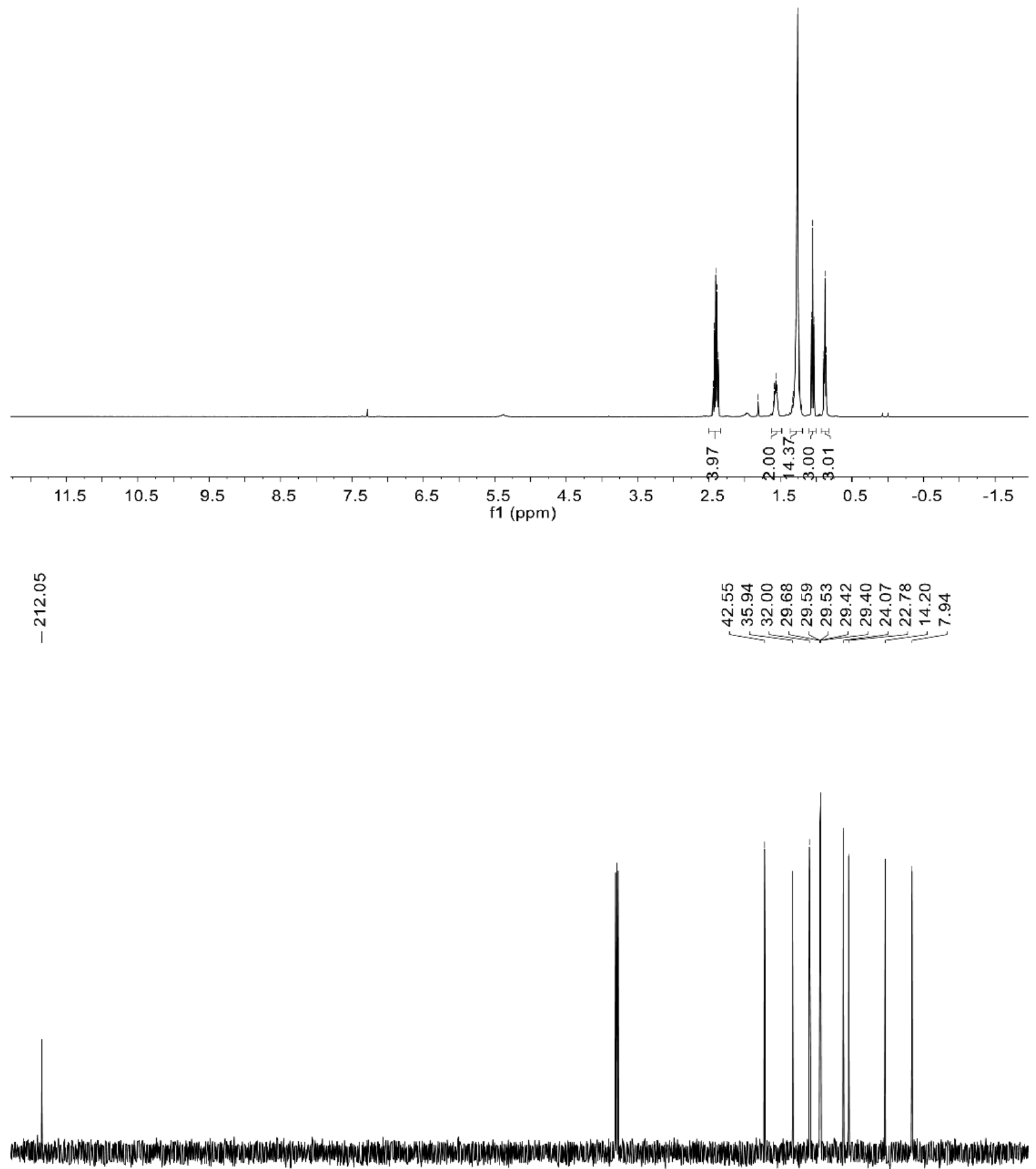

$\begin{array}{lllllllllllllllllllllll}210 & 200 & 190 & 180 & 170 & 160 & 150 & 140 & 130 & 120 & 110 \begin{array}{c}100 \\ \mathrm{f} 1\end{array}(\mathrm{ppm})\end{array}$ 


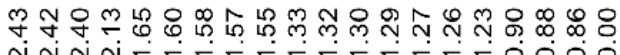

nhy4hththththo, tho
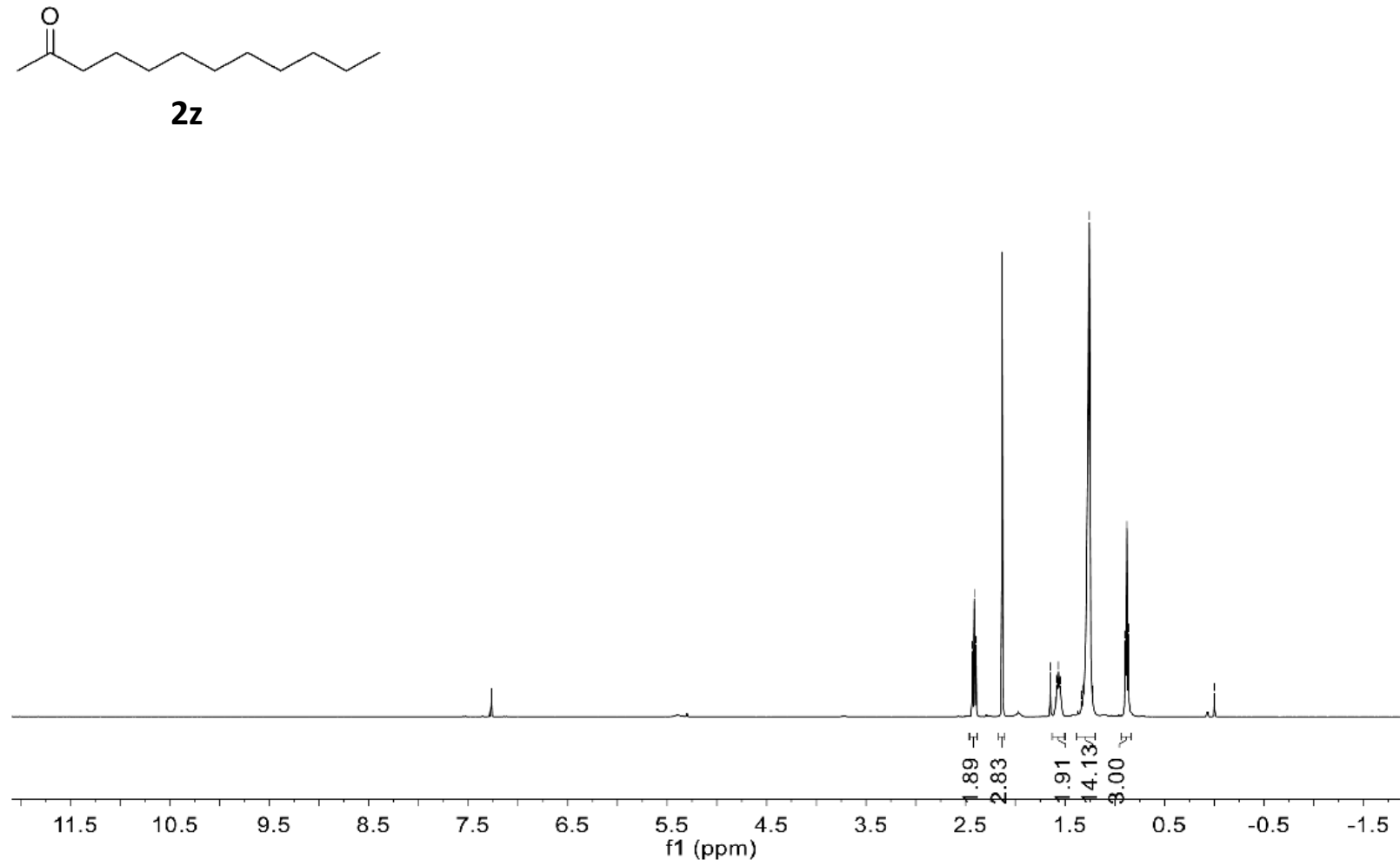

$\stackrel{\infty}{\circ}$

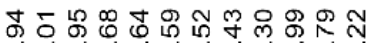

守 N

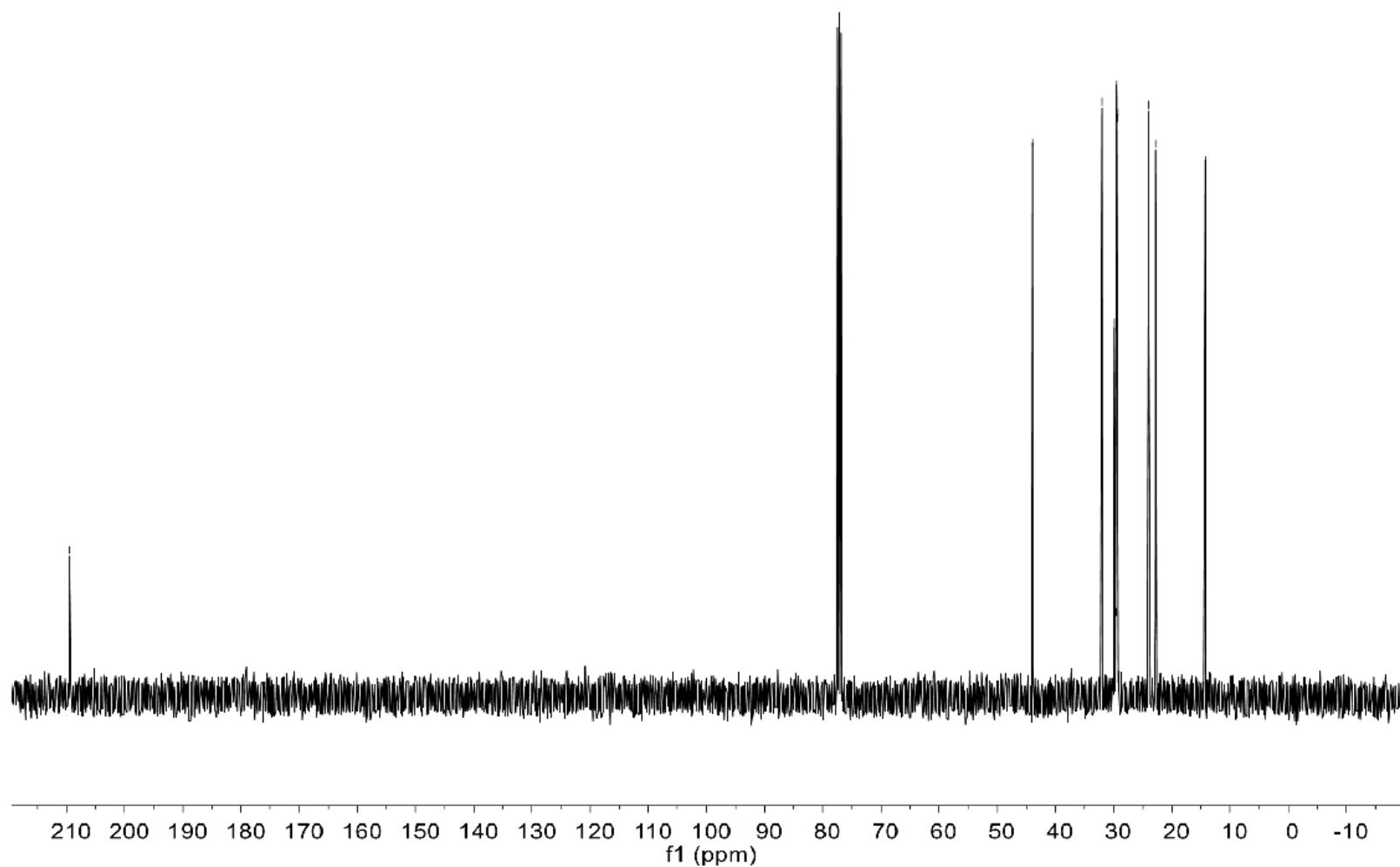




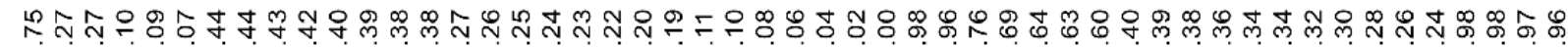

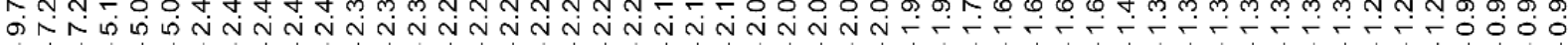<smiles>CC(C)=CCCC(C)CC=O</smiles>

$2 a a$
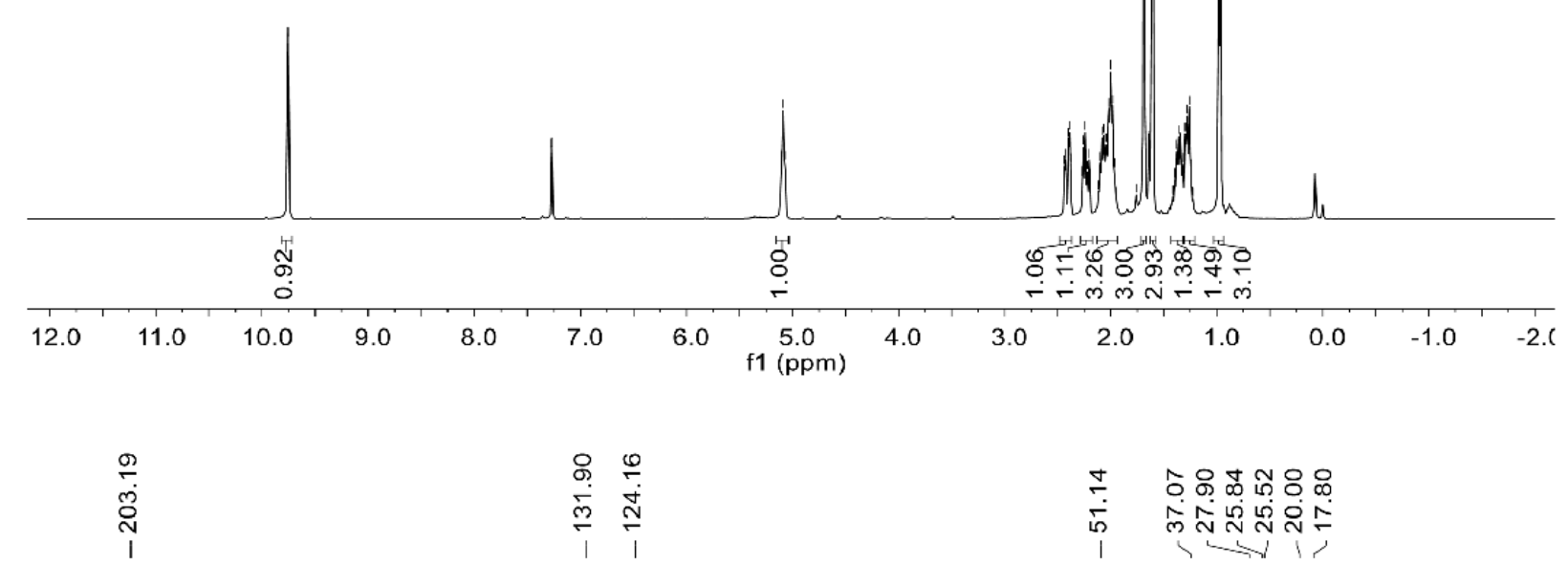

每

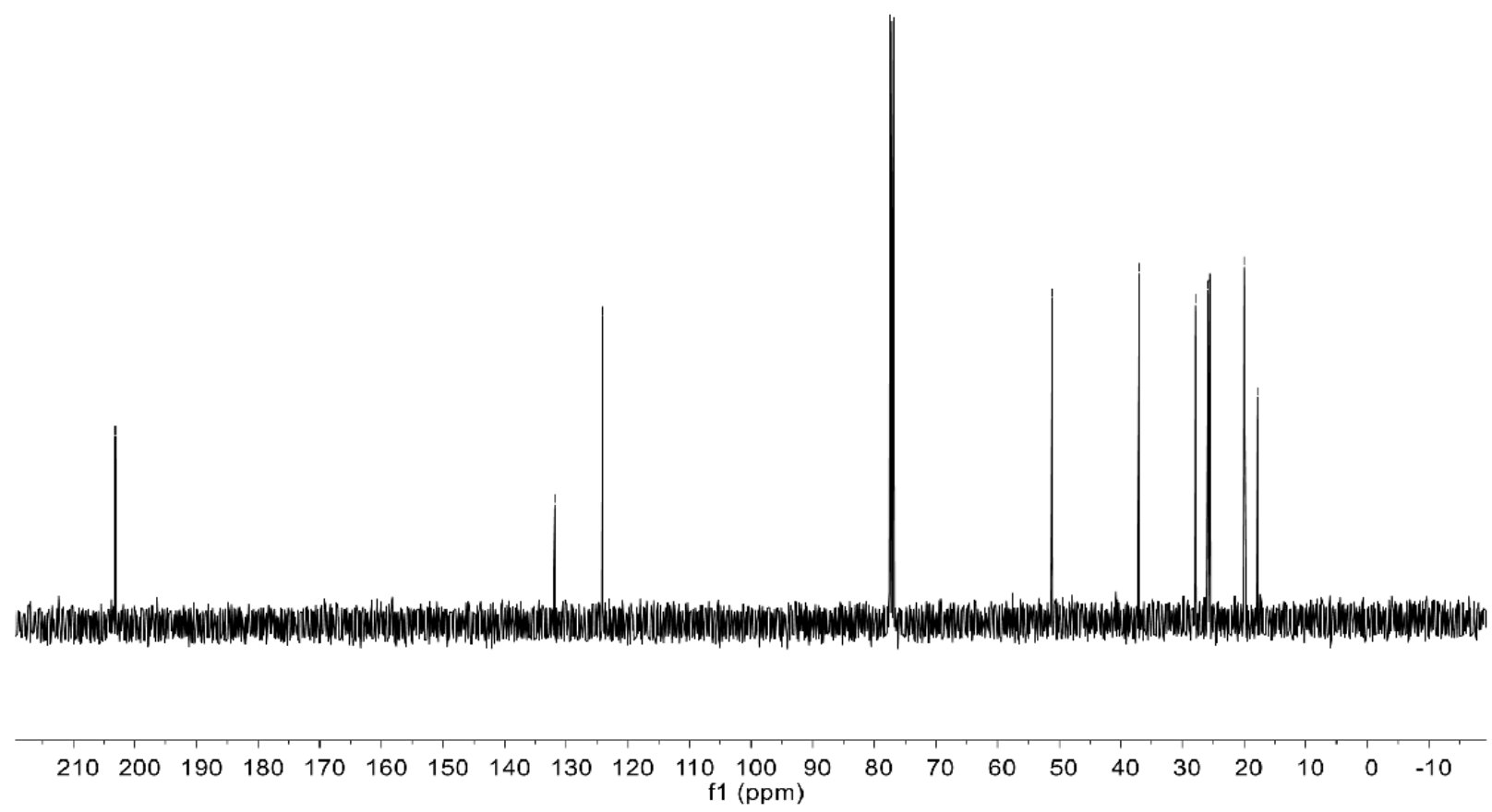


<smiles>CCCC1C[C@H]2CCc3cc(OC)ccc3[C@@H]2CCC1(C)C</smiles>

$2 a b$

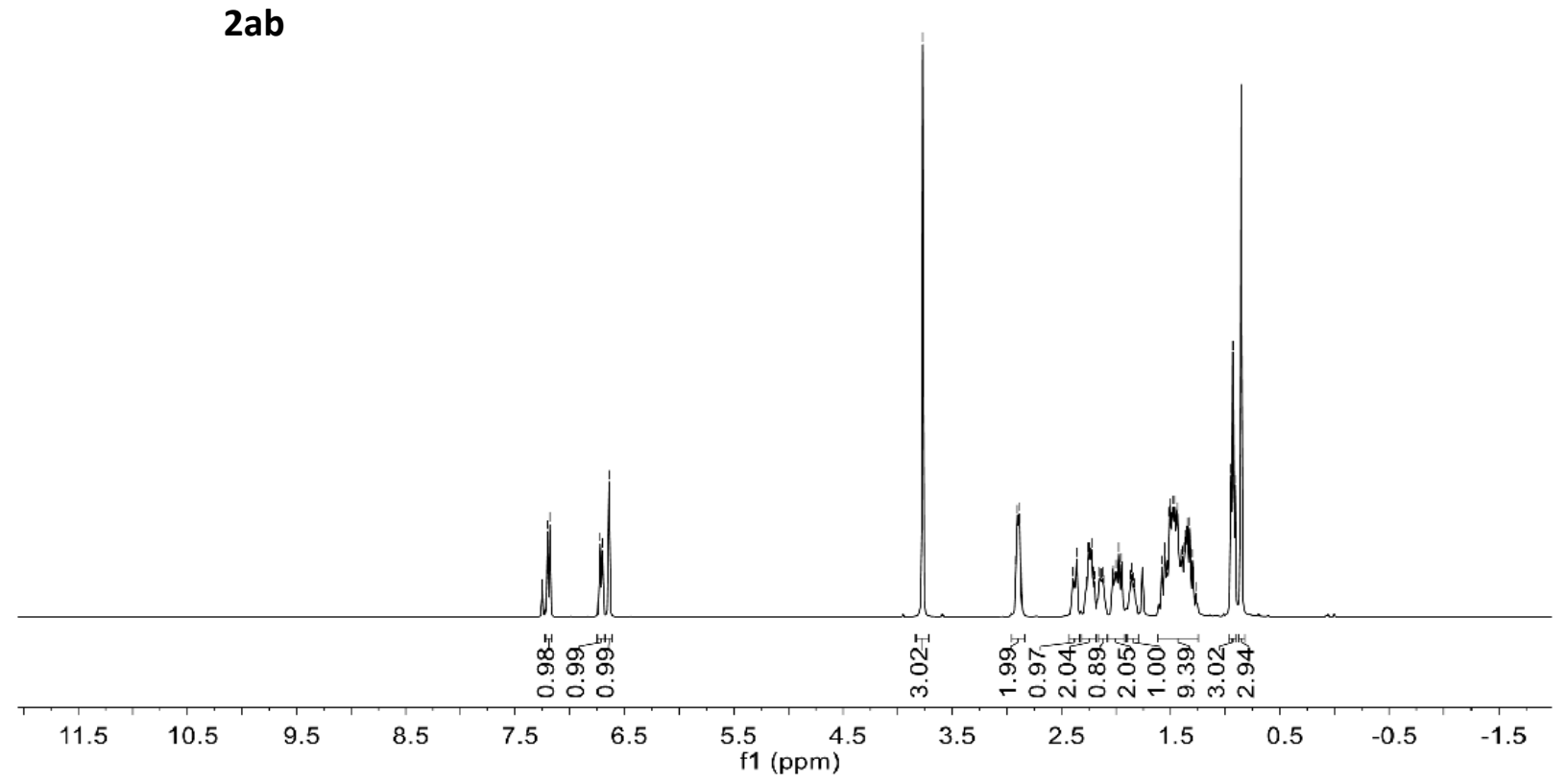

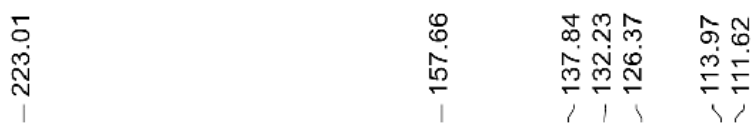

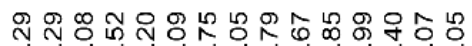

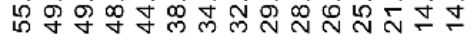

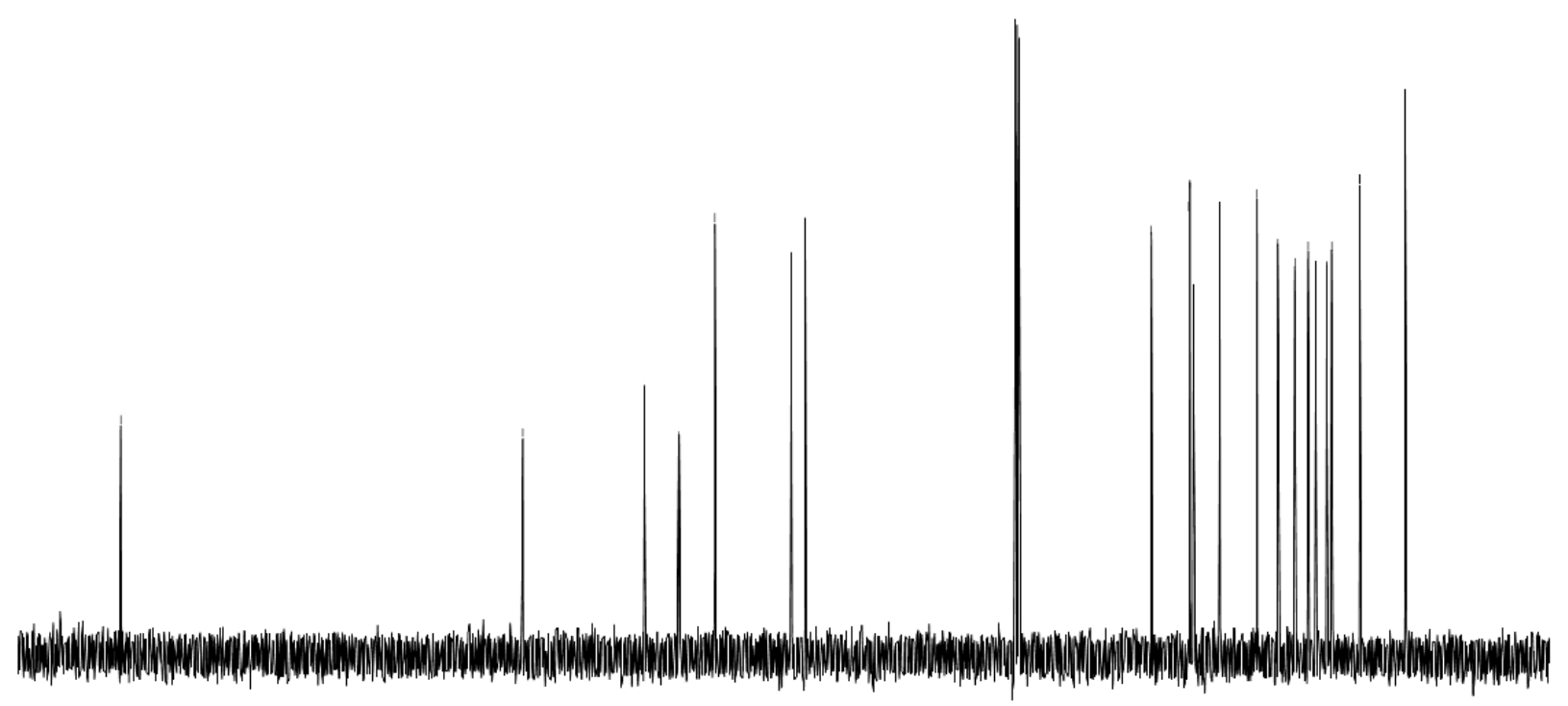

$\begin{array}{llllllllllllllllllllllll}230 & 220 & 210 & 200 & 190 & 180 & 170 & 160 & 150 & 140 & 130 & 120 & 110 & 100 & 90 & 80 & 70 & 60 & 50 & 40 & 30 & 20 & 10 & 0\end{array}$ f1 (ppm) 


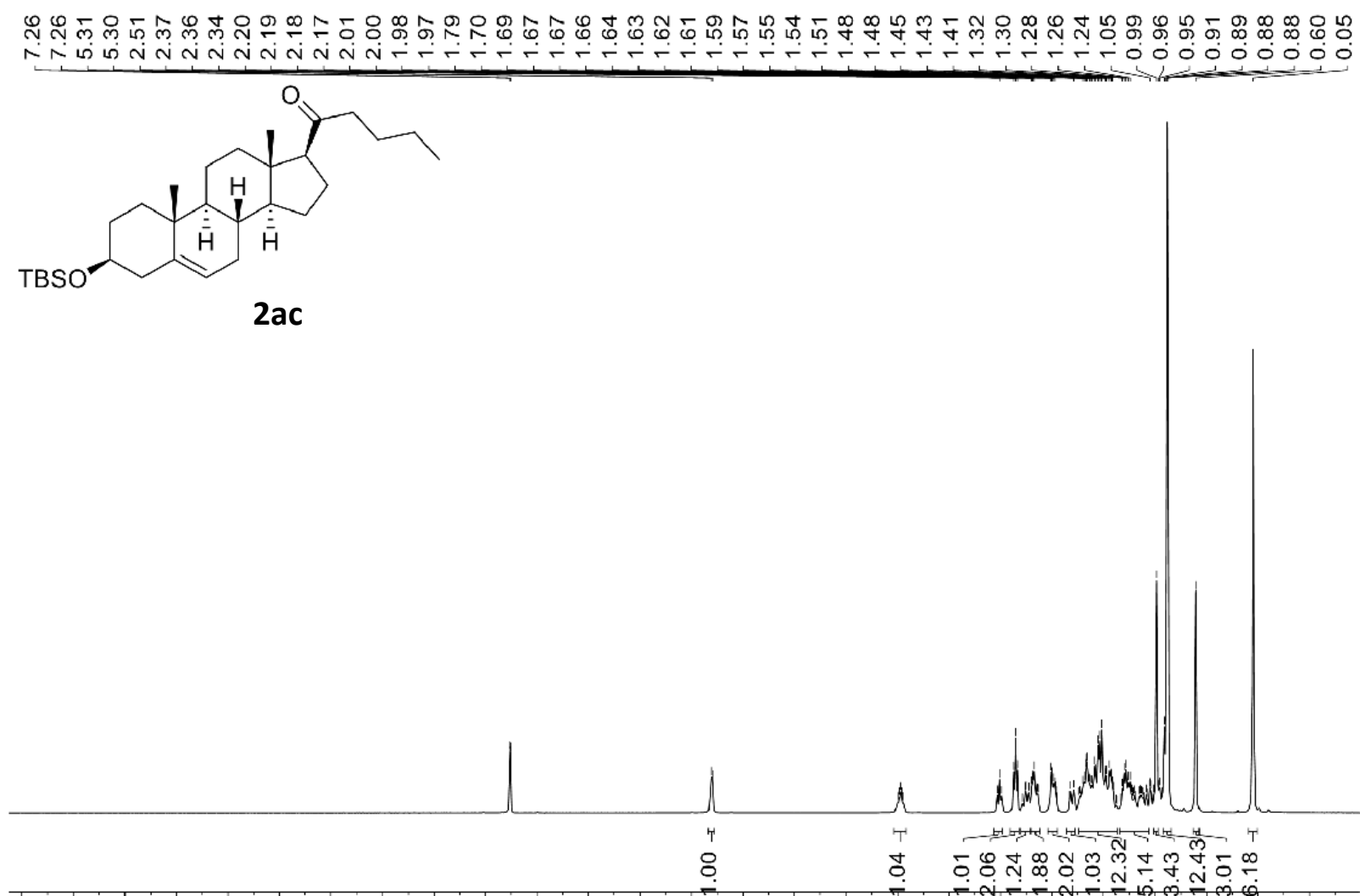

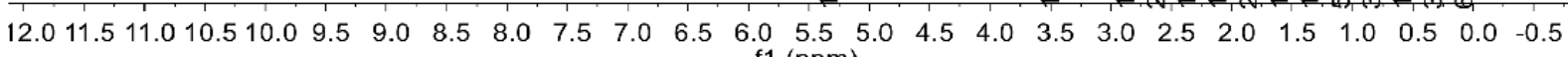
f1 (ppm)
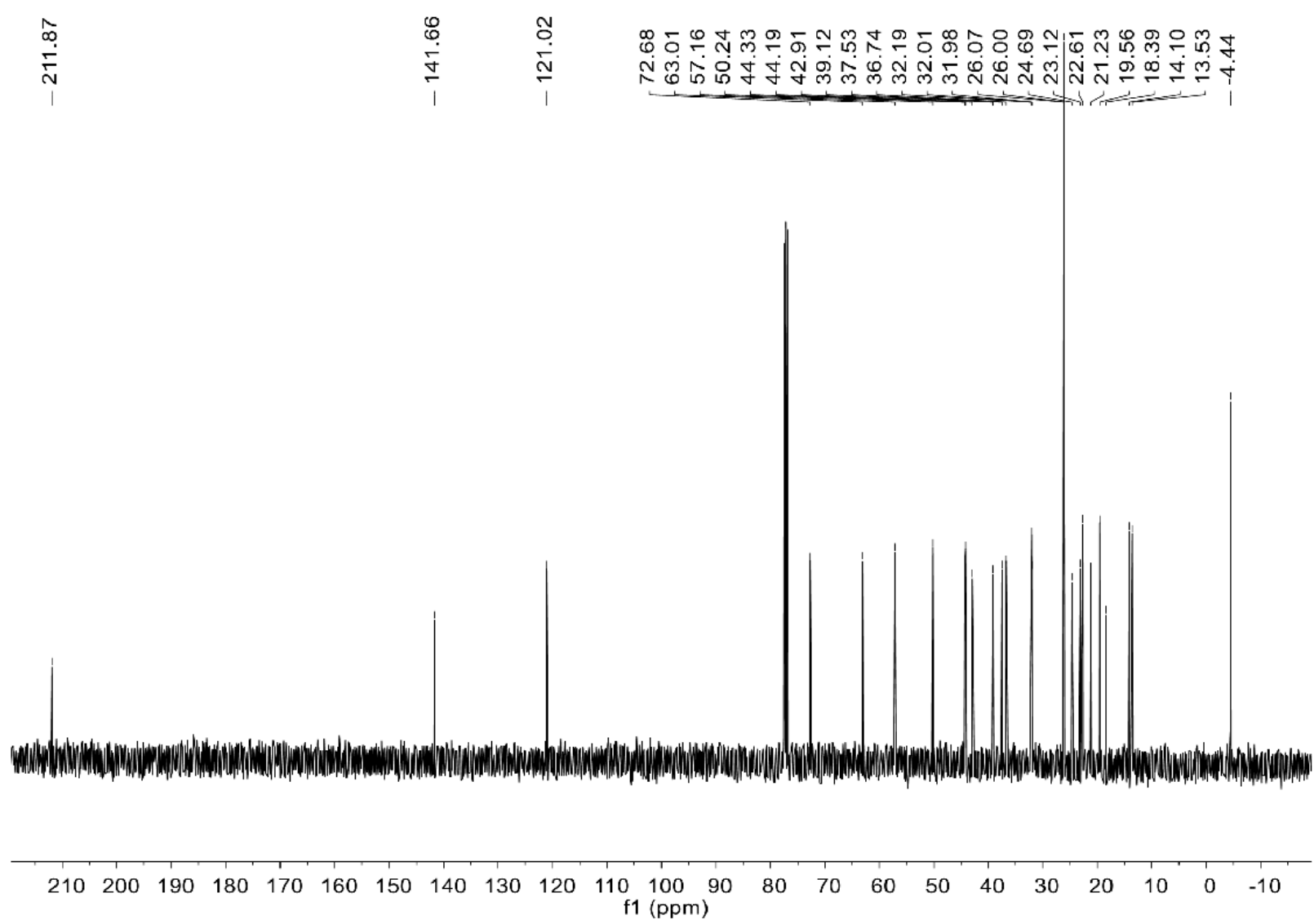


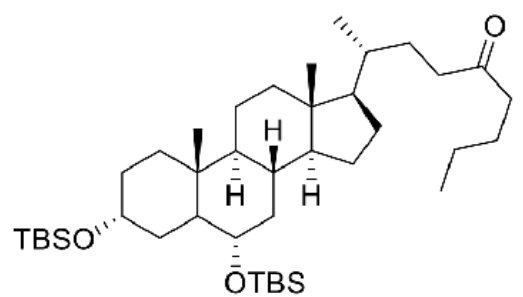

2ad

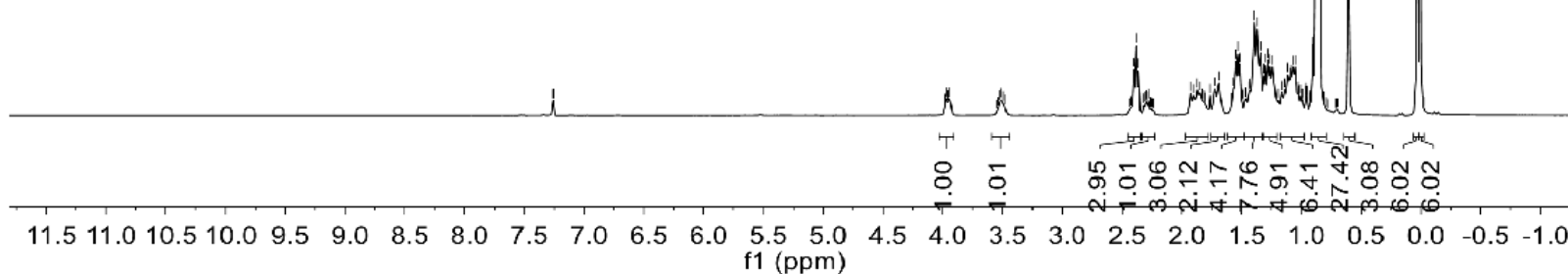




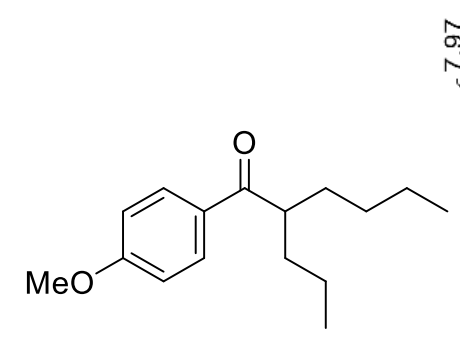

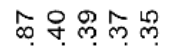

लंखल लंख

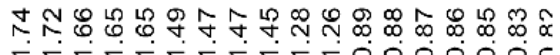

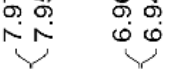

남

2ae

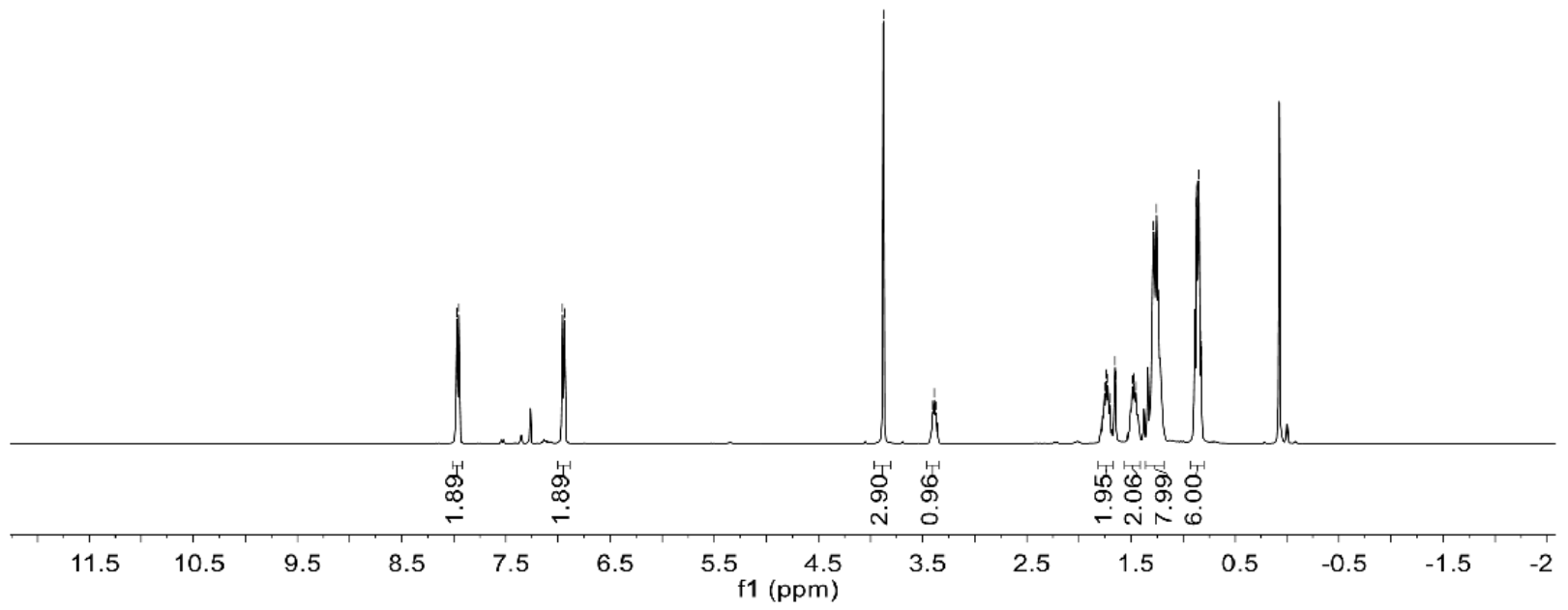

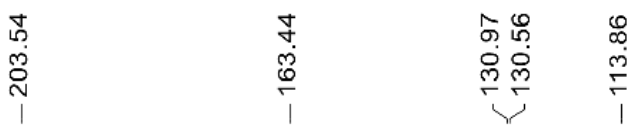

象

吕 守 फ्लm

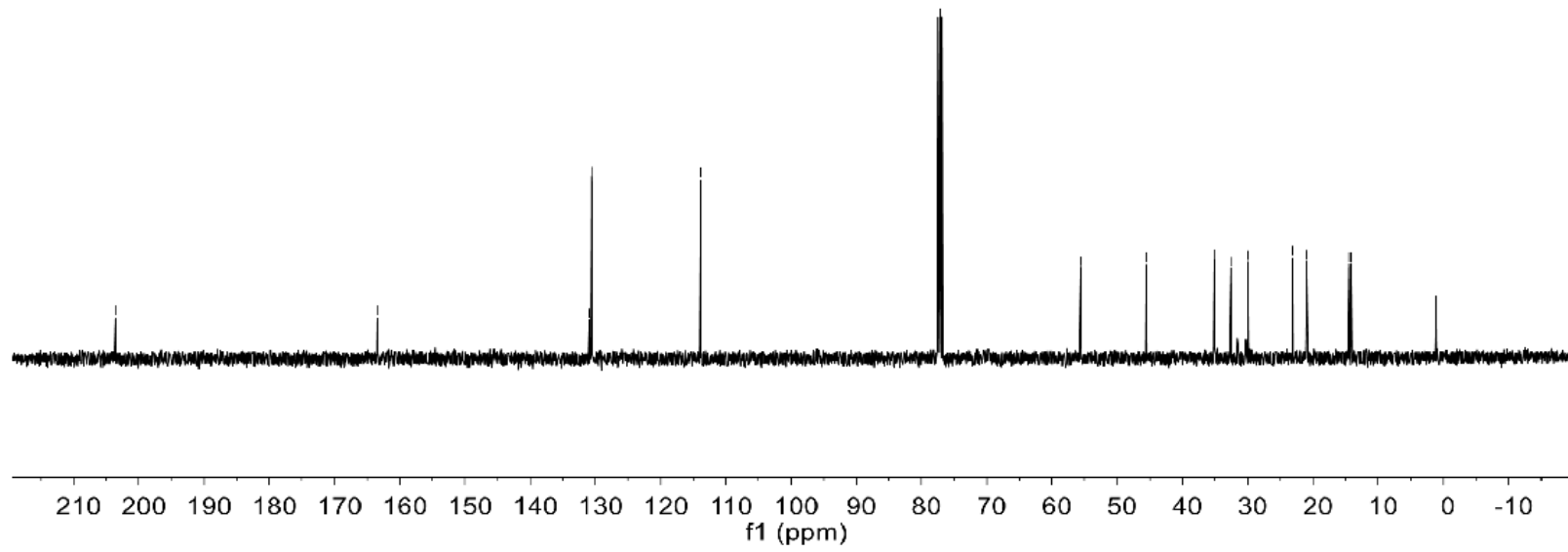

